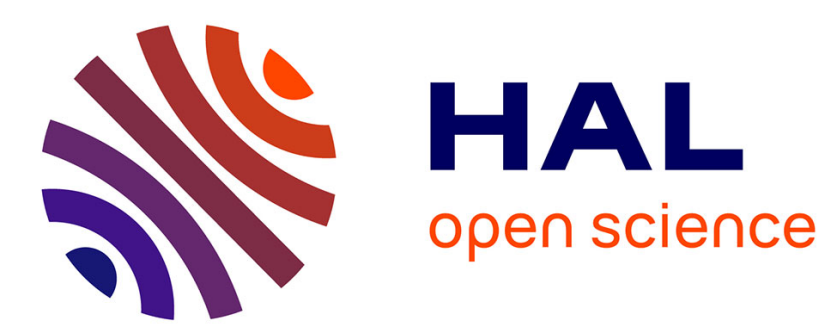

\title{
Imaging at High Angular Resolution of Stellar Surfaces and Close Environment
}

Nicolas Nardetto, Yveline Lebreton, Eric Lagadec

\section{To cite this version:}

Nicolas Nardetto, Yveline Lebreton, Eric Lagadec (Dir.). Imaging at High Angular Resolution of Stellar Surfaces and Close Environment. 2019. obspm-02318529

\section{HAL Id: obspm-02318529 https://hal-obspm.ccsd.cnrs.fr/obspm-02318529}

Submitted on 10 Dec 2020

HAL is a multi-disciplinary open access archive for the deposit and dissemination of scientific research documents, whether they are published or not. The documents may come from teaching and research institutions in France or abroad, or from public or private research centers.
L'archive ouverte pluridisciplinaire HAL, est destinée au dépôt et à la diffusion de documents scientifiques de niveau recherche, publiés ou non, émanant des établissements d'enseignement et de recherche français ou étrangers, des laboratoires publics ou privés. 


\section{EDP SCIENCES PROCEEDINGS}

\section{Imaging at High Angular Resolution of Stellar Surfaces and Close Environment}

Evry Schatzman School 2017

Nicolas Nardetto, Yveline Lebreton, and Eric Lagadec, Eds 
Printed in France

ISBN(print): 978-2-7598-2372-7 - ISBN(ebook): 978-2-7598-2373-4

All rights relative to translation, adaptation and reproduction by any means whatsoever are reserved, worldwide. In accordance with the terms of paragraphs 2 and 3 of Article 41 of the French Act dated March 11, 1957, "copies or reproductions reserved strictly for private use and not intended for collective use" and, on the other hand, analyses and short quotations for example or illustrative purposes, are allowed. Otherwise, "any representation or reproduction - whether in full or in part - without the consent of the author or of his successors or assigns, is unlawful" (Article 40, paragraph 1). Any representation or reproduction, by any means whatsoever, will therefore be deemed an infringement of copyright punishable under Articles 425 and following of the French Penal Code.

(C) EDP Sciences, 2019 


\section{Sponsors}

- Centre National de la Recherche Scientifique, France (CNRS, Formation Permanente)

- Programme National de Physique Stellaire (PNPS) of CNRS/INSU (Institut National des Sciences de l'Univers du CNRS)

- Centre National d'Etudes Spatiales (CNES, France)

- C4PO (Centre for Planetary Origin) (IDEX Université Côte d'Azur- JEDI)

- Action Spécifique Haute Résolution Angulaire (ASHRA, CNRS/INSU)

- Programme National de Planétologie (PNP, CNRS/INSU)

- Observatoire de la Côte d'Azur (OCA, France)

- Laboratoire Lagrange (UMR 7293, CNRS, OCA, Université Côte d'Azur)

- Jean-Marie Mariotti Centre (JMMC)

\section{Scientific Organizing Committee}

- N. Nardetto - Université Côte d'Azur, OCA, CNRS, Lagrange, F-06304 Nice, France

- Y. Lebreton - LESIA, Observatoire de Paris, PSL Research University, CNRS, Sorbonne Université, Univ. Paris Diderot, Sorbonne Paris Cité, Meudon 92195, France and Univ Rennes, CNRS, IPR (Institut de Physique de Rennes)-UMR 6251, F-35000 Rennes, France

- E. Lagadec - Université Côte d'Azur, OCA, CNRS, Lagrange, F-06304 Nice, France

- A. Meilland - Université Côte d'Azur, OCA, CNRS, Lagrange, F-06304 Nice, France

\section{Local Organizing Committee}

- N. Nardetto - Université Côte d'Azur, OCA, CNRS, Lagrange, F-06304 Nice, France

- Y. Lebreton - LESIA, Observatoire de Paris, PSL Research University, CNRS, Sorbonne Université, Univ. Paris Diderot, Sorbonne Paris Cité, Meudon 92195, France and Univ Rennes, CNRS, IPR (Institut de Physique de Rennes)-UMR 6251, F-35000 Rennes, France

- E. Lagadec - Université Côte d'Azur, OCA, CNRS, Lagrange, F-06304 Nice, France

- A. Meilland - Université Côte d'Azur, OCA, CNRS, Lagrange, F-06304 Nice, France

- G. Mella - Université Grenoble Alpes, CNRS, IPAG, F-38000 Grenoble, France

- I. Lapassat - Université Côte d'Azur, OCA, CNRS, Lagrange, F-06304 Nice, France 


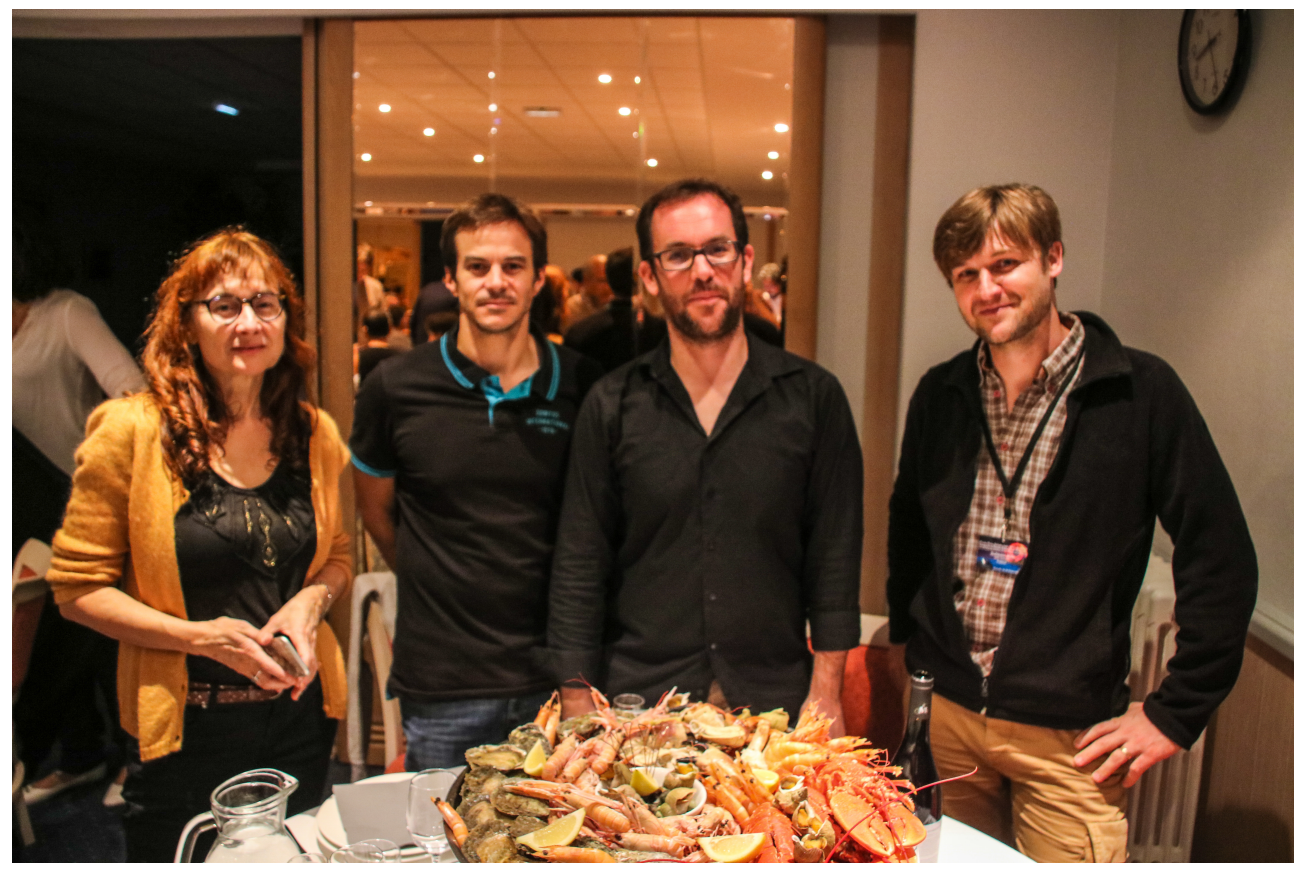

Figure 1. Some of the organisers of the school (from left to right: Yveline Lebreton, Nicolas Nardetto, Eric Lagadec and Guillaume Mella)

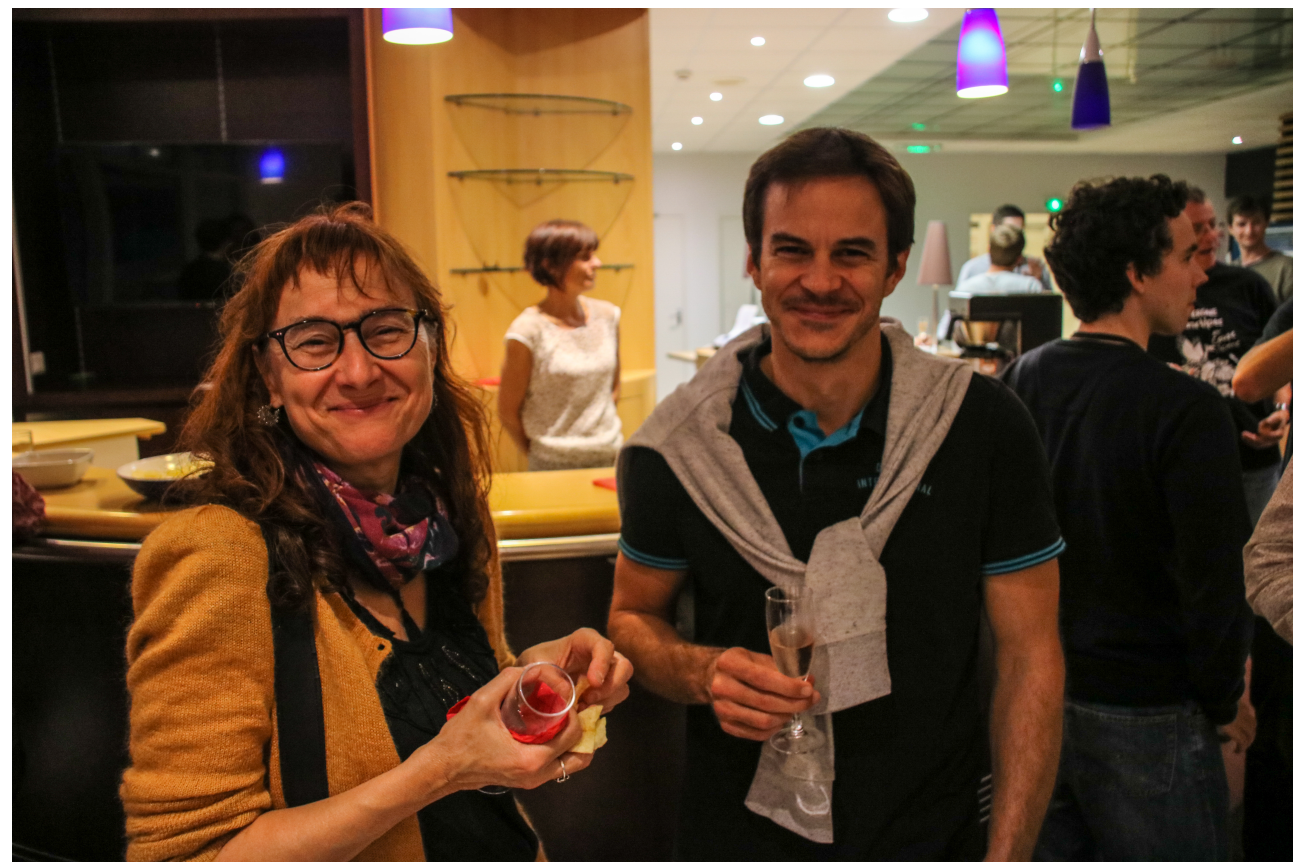

Figure 2. Yveline Lebreton et Nicolas Nardetto, enjoying the welcoming reception. 


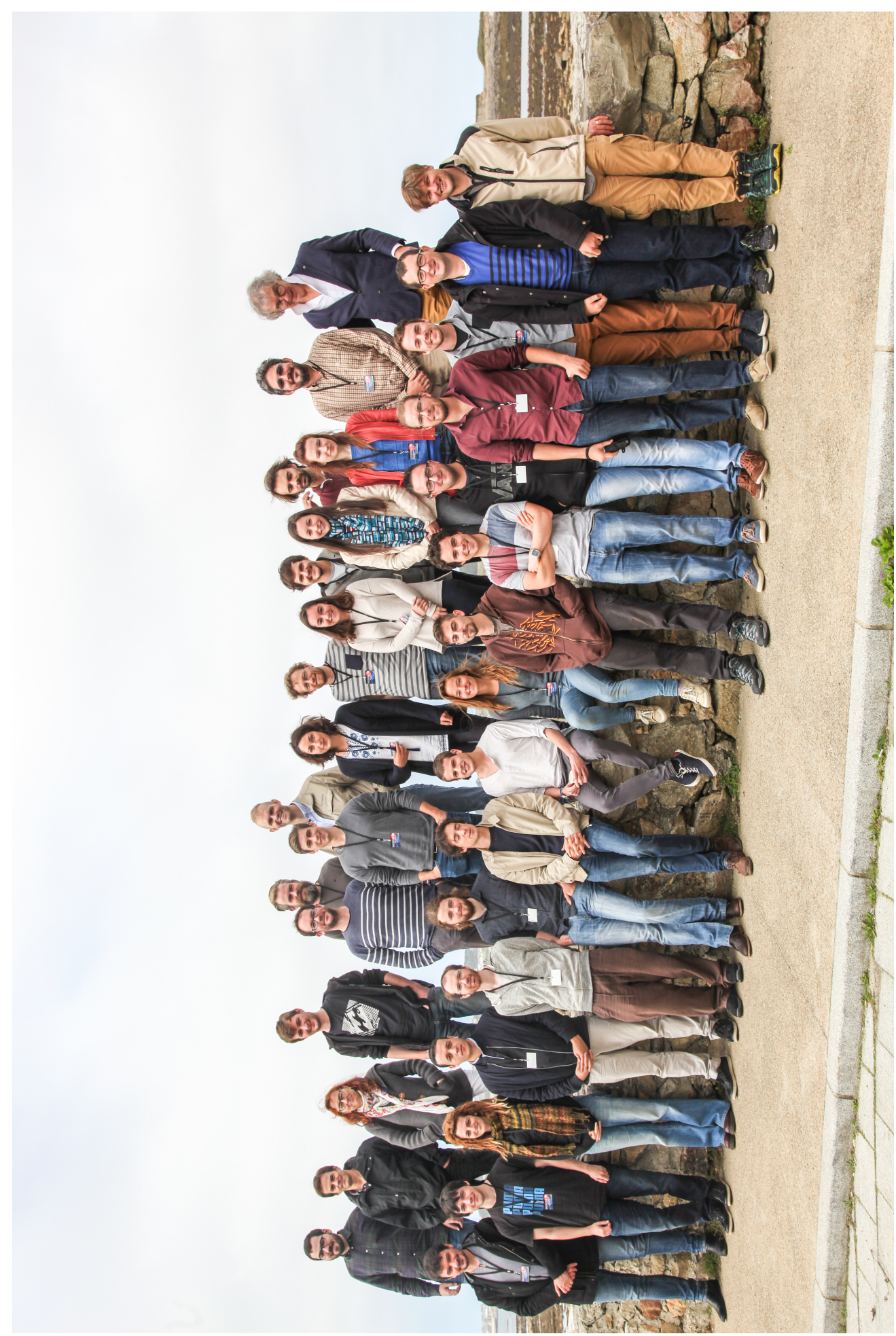

Figure 3. The EES17 participants. 


\section{List of participants}

1. Beldi Samir, GEPI, Observatoire de Paris, France

2. Bollen Dylan, Institute of astronomy, KU Leuven, Belgium

3. Borgniet Simon, CNRS UMI 3386 - Laboratoire Franco-Chilien d'Astronomie, Chile

4. Bouchaud Kevin, Laboratoire Lagrange, Observatoire de la Côte d'Azur, France

5. Ceau Alban, Laboratoire Lagrange, Observatoire de la Côte d'Azur, France

6. Crida Aurélien, Laboratoire Lagrange, Observatoire de la Côte d'Azur, France

7. Falla Romain, STAR Institute, Liège, Belgium

8. Fontanive Clémence, Institute for Astronomy, University of Edinburgh, Scotland

9. Hocdé Vincent, Laboratoire Lagrange, Observatoire de la Côte d'Azur, France

10. Jonak Juraj, Charles University, Faculty of Mathemathics and Physics, Czech Republic

11. Kervella Pierre, LESIA, Observatoire de Paris, France

12. Koumpia Evgenia, University of Leeds, UK

13. Kravchenko Kateryna, ULB Bruxelles, Belgium

14. Labdon Aaron, University of Exeter, Astrophysics Group, UK

15. Lagadec Eric, Laboratoire Lagrange, Observatoire de la Côte d'Azur, France

16. Lagrange Anne-Marie, Institut de Planétologie et d'Astrophysique de Grenoble, France

17. Lanthermann Cyprien, Institut de Planétologie et d'Astrophysique de Grenoble, France

18. Lebreton Yveline, LESIA, Observatoire de Paris et IPR, Université de Rennes 1, France

19. Lehmann Lucien, XLIM, Limoges, France

20. Ligi Roxanne, Laboratoire d'Astrophysique de Marseille, France

21. Martinache Frantz, Laboratoire Lagrange, Observatoire de la Côte d'Azur, France

22. Martinod Marc-Antoine, Laboratoire Lagrange, Observatoire de la Côte d'Azur, France

23. Maury Anaëlle, Département d'Astrophysique, CEA, Université Paris Saclay, France

24. Mella Guillaume, JMMC, Institut de Planétologie et d'Astrophysique de Grenoble, France

25. Ménard Francois, Institut de Planétologie et d'Astrophysique de Grenoble, France

26. Millour Florentin, Laboratoire Lagrange, Observatoire de la Côte d'Azur, France

27. Mourard Denis, Laboratoire Lagrange, Observatoire de la Côte d'Azur, France

28. Nardetto Nicolas, Laboratoire Lagrange, Observatoire de la Côte d'Azur, France 
29. Postnikova Marina, Laboratoire Lagrange, Observatoire de la Côte d'Azur, France

30. Potier Axel, LESIA, Observatoire de Paris, France

31. Rainot Alan, KU Leuven, Belgique

32. Royer Frédéric, GEPI, Observatoire de Paris, France

33. Saldanha da Gama de Almeida Elisson, Laboratoire Lagrange, Observatoire de la Côte d'Azur, France

34. Soulain Anthony, Laboratoire Lagrange, Observatoire de la Côte d'Azur, France

35. Surdej Jean, Université de Liège, Belgium

36. Trahin Boris, CNRS UMI 3386 - Laboratoire Franco-Chilien d'Astronomie, Chile

37. van der Plas Gerrit, Institut de Planétologie et d'Astrophysique de Grenoble, France

38. Villenave Marion, Institut de Planétologie et d'Astrophysique de Grenoble, France 


\section{Contents}

List of participants

$\begin{array}{ll}\text { Preface } & 11\end{array}$

I Diffraction-dominated observational astronomy 16

$\begin{array}{llr}1 & \text { Introduction } & 17\end{array}$

2 Images in astronomy $\quad 18$

3 Coherence properties of light 19

4 Diffraction-dominated imaging 25

5 High-contrast imaging $\quad 29$

5.1 Pupil apodization . . . . . . . . . . . . . . . . . 30

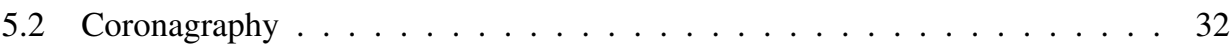

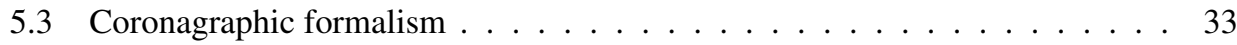

6 Atmospheric turbulence and Adaptive Optics $\quad 34$

7 Extreme adaptive optics $\quad 40$

8 Calibration of biases $\quad 42$

9 Focal-plane based wavefront control? $\quad 44$

10 Conclusion $\quad 50$

II Introduction to optical/IR interferometry: history and basic principles $\quad 55$

1 Introduction $\quad 56$

2 Some reminders $\quad \mathbf{5 8}$

2.1 Complex representation of an electromagnetic wave . . . . . . . . . . . 60

2.2 Principle of Huygens-Fresnel f. . . . . . . . . . . . . 62

3 Brief history about the measurements of stellar diameters $\quad \mathbf{6 4}$

3.1 Galileo . . . . . . . . . . . . . . . . . . . . . 64

3.2 Newton . . . . . . . . . . . . . . . . . . . . . . . 64

3.3 Fizeau-type interferometry . . . . . . . . . . . . . . . . . . . 64

3.4 Home experiments: visualization of the Airy disk and the Young interference

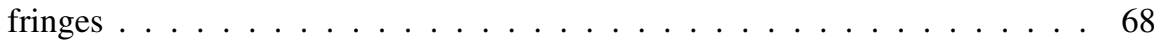


4 Light coherence $\quad \mathbf{7 0}$

4.1 Quasi-monochromatic light . . . . . . . . . . . . . . . . 70

4.2 Visibility of the interference fringes . . . . . . . . . . . . . 71

4.3 Spatial coherence . . . . . . . . . . . . . . . . . . . . 73

4.4 Zernicke-van Cittert theorem . . . . . . . . . . . . . . . . . . . . . . . 74

4.5 Some remarkable properties of the Fourier transform and applications . . . . 75

$4.5 .1 \quad$ Linearity: . . . . . . . . . . . . . . . . . . 76

4.5.2 Symmetry and parity: . . . . . . . . . . . . . 76

4.5 .3 Similarity: . . . . . . . . . . . . . . . 76

4.5.4 Translation: . . . . . . . . . . . . . . . . . . . . 77

4.5.5 Door function: . . . . . . . . . . . . . . . . . . . 77

4.5.6 Distribution of Dirac: . . . . . . . . . . . . . . . . . . . . . 77

4.5.7 Applications: . . . . . . . . . . . . . . . 78

5 Some examples of interferometers $\quad 80$

6 Three important theorems and some applications $\quad 83$

6.1 The fundamental theorem: relation between the pupil and focal planes . . . . 84

6.1.1 Applications of the fundamental theorem: the case of a single square aperture ...................... 87

6.1.2 Applications of the fundamental theorem: the case of a circular aperture 89

6.1.3 Applications of the fundamental theorem: the two telescope interferometer . . . . . . . . . . . . . . . . . 90

6.1.4 Other types of beam recombination . . . . . . . . . . . . 91

6.2 The convolution theorem . . . . . . . . . . . . . . . . 96

6.2.1 Application to the case of the two telescope interferometer . . . . . . 97

6.2.2 Interferometric observations of a circular symmetric source . . . . . . 98

6.3 The Wiener-Khinchin theorem . . . . . . . . . . . . . . . . . . . . . 99

6.4 Appendix . . . . . . . . . . . . . . . . . . . 100

III Optical Long Baseline Interferometry 104

1 Introduction $\quad 105$

2 Angular diameter and coherence of the wavefront 106

3 Angular diameter and Object-Image relationship $\quad 108$

4 A direct imaging technique $\quad 111$

5 The reality of the coherence $\quad 114$

6 Practical considerations for the implementation of an interferometer $\quad 117$

7 A modern panorama of optical long baseline interferometry 120

$7.1 \quad$ NPOI . . . . . . . . . . . . . . . . . . . . . 120

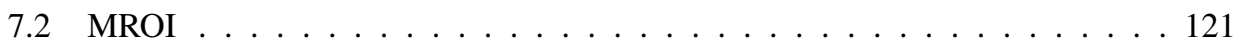

7.3 The VLTI array . . . . . . . . . . . . . . . . . . . 121

7.4 The CHARA array . . . . . . . . . . . . . . . . . 122 
8 The study of stellar surface and their close environment using interferometry $\mathbf{1 2 3}$

8.1 Imaging at very high angular resolution . . . . . . . . . . . . . 123

8.1.1 Giant and red supergiants stars . . . . . . . . . . . . . . . . . 124

8.1 .2 The star/planet interaction . . . . . . . . . . . . . . 126

8.1 .3 The rotating stars . . . . . . . . . . . . . . . . . 127

8.2 Cepheids and eclipsing binaries as distance indicators in the universe . . . . . 129

8.3 Young stellar objects . . . . . . . . . . . . . . . . . . . . 130

8.4 The prototypic $\operatorname{star} \beta$ Lyrae . . . . . . . . . . . . . . . . . . . . . 132

8.5 Interferometry, asteroseismology and fundamental stellar and planetary parameters . . . . . . . . . . . . . . . . . . . 133

8.6 Doppler Imaging and Hot Jupiter Detection . . . . . . . . . . . . . . 136

8.7 Detection of the gravitational redshift in the orbit of the star S2 near the Galactic center massive black hole . . . . . . . . . . . . . . . 138

9 Steps to the future 


\section{Preface}

The 2017 edition of the Evry Schatzman school (EES 2017) of the French National Program for Stellar Physics (PNPS) was held in Roscoff (France) from the 24th to the 29th of September ${ }^{1}$. This school focused on high angular resolution imaging. High angular resolution is a thriving theme with many operating instruments such as SPHERE (VLT) and ALMA, but also long-term projects, like the forthcoming JWST and ELT. It concerns monolithic telescopes, but also interferometric instruments with for instance, PIONIER (VLTI), MIRC (CHARA), VEGA (CHARA), GRAVITY (VLTI), MATISSE (VLTI) ..., or at longer wavelengths ALMA and NOEMA. All these instruments, with their specific angular resolution, their wavelength coverage and spectral resolution, are essential for studying star forming regions, protoplanetary discs, as well as the surface and environment of stars. High angular resolution imaging is a priority theme for the National Program for Stellar Physics (PNPS) and is also essential for the study of extrasolar systems, a unifying theme of the French National Program for Planetology (PNP).

The research theme of "High Angular Resolution" is today structured in France by the ASHRA (Action Spécifique pour la Haute Résolution Angulaire) specific action. Research in the High Angular Resolution domain aims at a single objective: the control of the wave front to reach the limit of resolution. Around this objective, the discipline has been regularly diversified by the successive apparition of several research themes during its history: optical interferometry, adaptive optics, very high dynamics, signal processing, not to forget the study of atmospheric turbulence, and the development of innovative optical concepts.

The impact of optical interferometry on stellar physics (but not only) is extremely diverse. We can mention the determination of fundamental stellar and exoplanetary parameters, asteroseismology, the distance to Cepheids, the study of protoplanetary disks, fast rotators, the convective structure of (super)giant stars and their circumstellar environments. Interferometry has produced images since about 2009. At the same time, thanks to the development of adaptive optics, it is now possible to directly image the very close environment of giants and red supergiants (like Betelgeuse or L2 Puppis) with monolithic telescopes (such as NACO at the VLT, for example). These revealed the presence of asymmetric nebula due to nonisotropic, transient mass loss. SPHERE (VLT)is currently producing similar results. In the longer term, direct imaging of stellar surfaces will be possible with the ELT, leading to a better understanding of stellar convection, the environment of massive or evolved stars together with their mass loss, etc...

High angular resolution requires state-of-the-art instrumental expertise, which must take into account a whole range of technical and scientific constraints. The rapid evolution of this theme in the world of stellar and planetary physics raises the need of a good communication between several communities: high angular resolution instrument designers on the one hand and their users on the other, i.e. stellar-physicists and/or exo-planetologists, for instance. This school thus aimed first and foremost to bring these two communities close together. There are also strong emerging synergies between the different imaging techniques: monolithic telescopes versus interferometry, optical versus radio observations, and we can also mention, for instance, the fruitful complementarity of interferometry and asteroseismology, in order to determine the fundamental parameters of stars. The school aimed at clarifying these synergies. It was also a matter of "democratizing" the high angular resolution techniques in order to make this theme more accessible to the entire "stellar" and "planetary" community.

The EES 2017 school gathered 38 participants, including 1 master student, 20 doctoral students, 3 post-docs, 1 computer engineer, and 13 researchers, including 10 speakers/organizers. The proportion of students and post-docs was therefore significant (around

\footnotetext{
${ }^{1}$ https://hraetoile2017.sciencesconf.org/program
} 
63\%). Seven participants were coming from European, non-French, laboratories. The school was organized around five three-hour courses, three four-hour sessions, and two thematic seminars.

The first lecture, by Frantz Martinache, was dedicated to the principles of imaging together with a practical session on atmospheric disturbances and image processing techniques. The second course, by Anne-Marie-Lagrange, ${ }^{2}$ gave an overview of current imaging instruments with the latest applications both in terms of planetology (protoplanetary disks, detections of exoplanets) and in terms of stellar physics (environment of evolved stars ...). The corresponding practical session was devoted to SPHERE/VLT data processing. The first part of the school on imaging (mostly related to monolithic instruments) finally ended with perspectives, and a seminar on the ELT. The third course, by Jean Surdej, introduced the complex principles of interferometry based on the imaging principles enunciated in the first lecture. The fourth lecture, by Denis Mourard, continued with an overview of current optical interferometric instruments and the latest spectacular astrophysical results, including in particular the determination of fundamental stellar and planetary parameters. The associated practical session was based on the tools of the JMMC (Center Jean-Marie Mariotti, French structure offering user support for all the interferometric instruments of the world), with also an example of data processing and image reconstruction in interferometry. The session on optical interferometry ended with a seminar on the most recent instrument, Gravity on the VLTI. The school finished with a fifth lecture on radio interferometry, by Anaëlle Maury, which was an important complement to previous lectures ${ }^{2}$.

The school thus made it possible to understand that interferometry is ultimately an imaging technique, and that radio interferometry, which is 30 years older than optical interferometry, has benefited from the fact that it is easier to determine the fringe position (phase) in this wavelength range, compared to the optical domain. This also allows for a more direct image reconstruction. The overview of the astrophysical applications associated with these different techniques was very complete, with a good balance (and interesting links) between proto-planetary disks, exo-planets and stellar environments. In addition, the three practical sessions, highly appreciated, have really made it possible to give practical tools to the students for this sometimes very complex knowledge on imaging techniques. These sessions also allowed a lot of exchanges among the participants in a rather relaxed atmosphere. In addition to classes, lectures and seminars, a discussion session around a few posters was organized to stimulate communication and questions.

We hope that this book will be useful for the students but also engineers and researchers who attended the school, and to anyone who wants to learn about the fundamental principles of imaging and interferometry, as well as the latest astrophysical applications. The field of Imaging and High Angular Resolution in stellar physics is a very active field of research, which gives spectacular results, in the sense that they can be of interest also to the public. Let us mention to finish the famous star peanut which had, in its time, opened the astro-appetite of the public, especially in the French Riviera ! This result was obtained by our colleague Olivier Chesneau who passed away the 17th of May 2014, specialist of interferometry. We dedicate this school and this book to him and his memory.

We warmly thank our colleagues of the Scientific and Local Organizing Committees, Anthony Meilland for the design of the website of the school and the poster, Guillaume Mella for his very careful and expert handling of the practical sessions, and Isabelle Lapassat for managing the administrative aspects. We warmly thank Anna Surdej who made a careful video-recording of the talks and took many photos. We are also grateful to everyone from the staff of the Station Biologique de Roscoff for their warm welcome and help during the

${ }^{2}$ Unfortunately, this lecture has not been reproduced in the book 
school. Last but not least, we warmly thank the lecturers Frantz Martinache, Jean Surdej, Denis Mourard, Anne-Marie Lagrange, Annaëlle Maury, and Pierre Kervella for their comprehensive lectures and enthusiasm, which made the school a success.

Nicolas Nardetto, Yveline Lebreton \& Eric Lagadec

- EES 2017 co-chairs

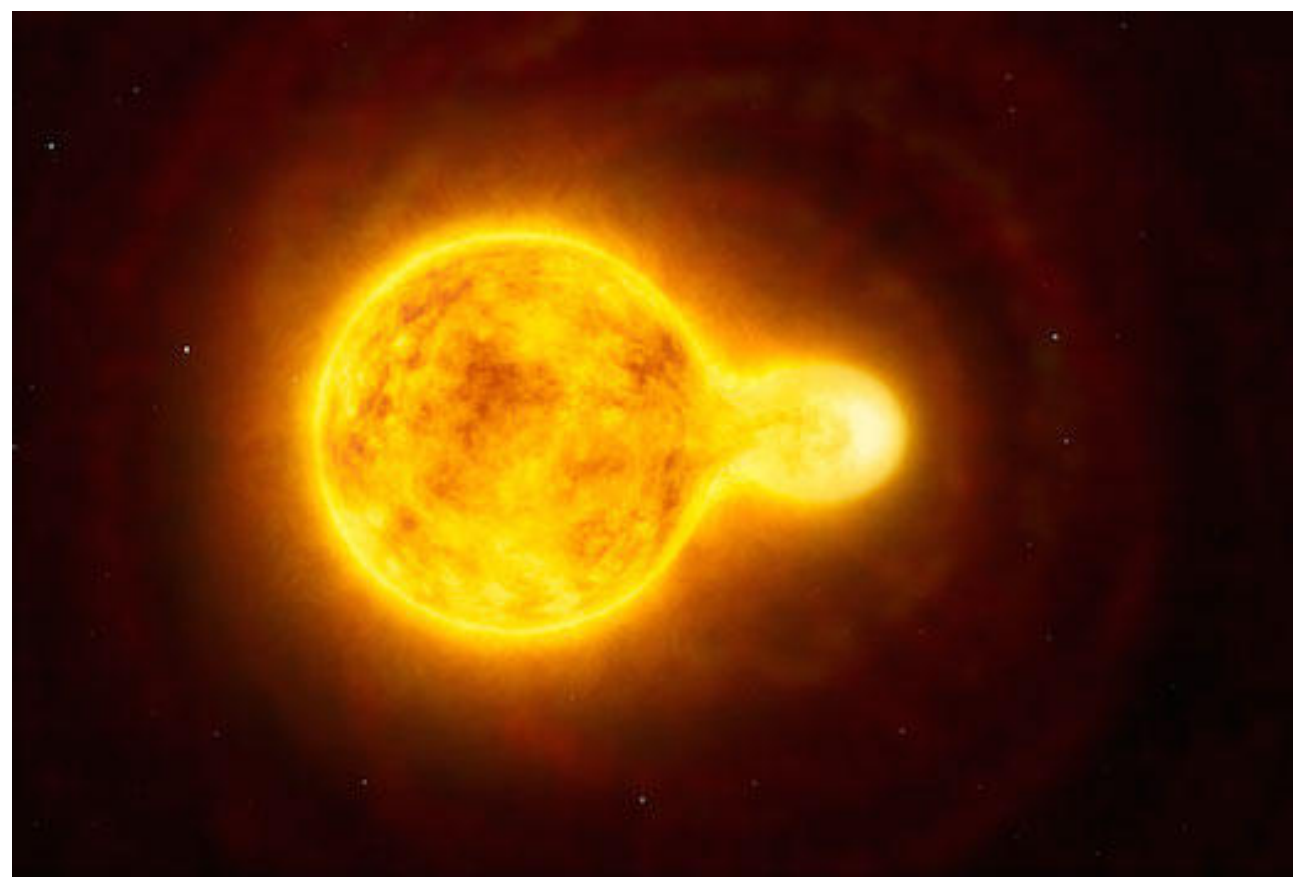

Figure 4. Artist's impression of the yellow hypergiant HR5171, a.k.a. the peanut Nebula, as mapped by our late colleague Olivier Chesneau. Olivier was a very active member of the high angular resolution community and the observations he obtained of this object are a perfect example of what high angular resolution can teach us about stellar physics. 


\section{Ecole Evry Schatzman 2017 du PNpS}

Imagerie à baute Résolution Angulaire des Surfaces Stellaires et de leur Environnement Proche

Comité Scientifique

N. Nardetto (OCA, chair)

y. Lebreton (LeSIA)

e. Lagadec (OCA)

Comité d'Organisation

Comité d'Organisation
N. Nardetto (OCA, chair)

N. Nardetto (OCA, ch
Y. Lebreton (LeSIA)

e. Lagadec (OCA)

A. Meilland (OCA

1. Lapassat (OCA)

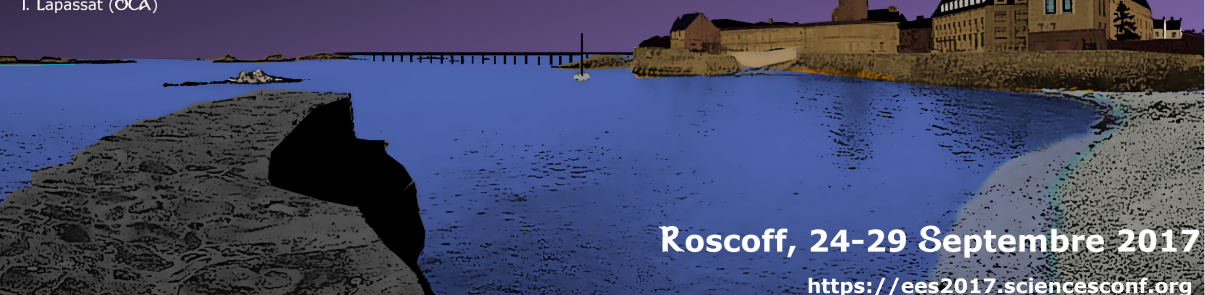

https:/ / ees 2017/sciendesoliforg

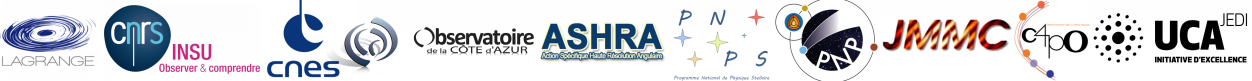

Figure 5. Poster of the 2017 Evry Schatzman School (credit: Anthony Meilland, OCA). 


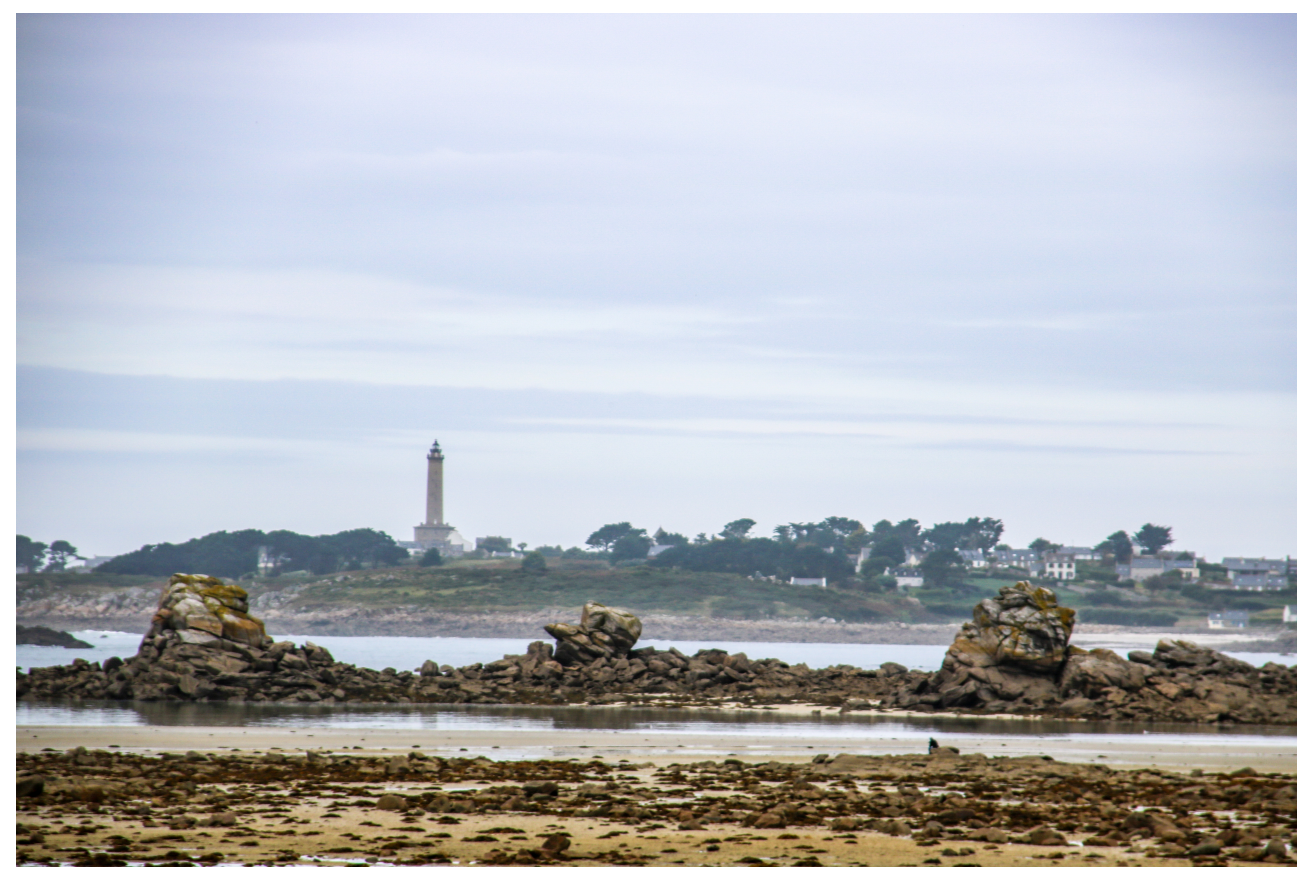

Figure 6. Low tide in Roscoff (Brittany, France), looking at the Batz Island, a few kilometers away. 


\section{Diffraction-dominated observational astronomy}




\title{
Diffraction-dominated observational astronomy
}

\author{
Frantz Martinache ${ }^{1, * * *}$ \\ ${ }^{1}$ Laboratoire Lagrange, Université Côte d'Azur, Observatoire de la Côte d'Azur, CNRS.
}

\begin{abstract}
This paper is based on the opening lecture given at the 2017 edition of the Evry Schatzman school on high-angular resolution imaging of stars and their direct environment. Two relevant observing techniques: long baseline interferometry and adaptive optics fed high-contrast imaging produce data whose overall aspect is dominated by the phenomenon of diffraction. The proper interpretation of such data requires an understanding of the coherence properties of astrophysical sources, that is, the ability of light to produce interferences. This theory is used to describe high-contrast imaging in more details. The paper introduces the rationale for ideas such as apodization and coronagraphy and describes how they interact with adaptive optics. The incredible precision brought by the latest generation adaptive optics systems makes observations particularly sensitive to subtle instrumental biases that must be accounted for, up until now using post-processing techniques. The ability to directly measure the coherence of the light in the focal plane of high-contrast imaging instruments using focal-plane based wavefront control techniques will be the next step to further enhance our ability to directly detect extrasolar planets.
\end{abstract}

\section{Introduction}

This edition of the Evry Schatzman school is dedicated to the high angular resolution imaging of the surface of stars and their direct environment. Two families of observational techniques: adaptive-optics (AO) assisted high-contrast imaging and long baseline interferometry, are contributing to making this ambition a reality.

As different as they may seem at first look (see Figure 7), the data produced by these observational techniques share many characteristics. In both cases, whether it be interference fringes or images boosted by a high-order $\mathrm{AO}$ system, these data are dominated by diffraction features, that are the combined signature of the telescope and instrumentation used to perform the observations, and include the effect of ever changing atmospheric conditions. The electromagnetic nature of the light collected by the observatory, which can oftentimes be neglected when looking at wide-field images, becomes manifest with these observing techniques since features such as diffraction rings, fringes and speckles become prominent.

For each structure present in the data, one must be able to discriminate the signature of a genuine structure like that of a faint planetary companion, a clump in a circumstellar disk, or a structure of a stellar surface, from a diffraction feature. The ultimate discrimination criterion has to do with the degree of coherence of the structure in question.

Figure 7 presents two examples of diffraction dominated frames, one produced by a single telescope, the other by an interferometer. In both cases, the question one needs to examinate

***e-mail: frantz.martinache@oca.eu 
is: is the object I am looking at a point source or did my frame capture the presence of more complex structures? To figure out how to answer this question, we need to take a closer look at the process of image formation.
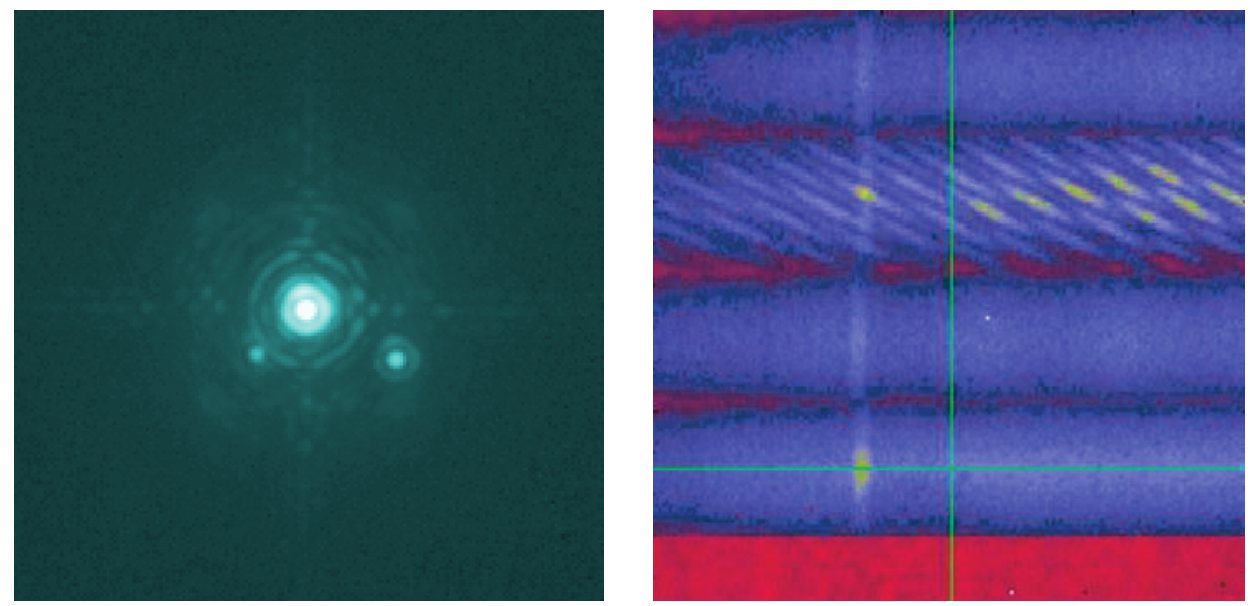

Figure 7. Two examples of diffraction-dominated data: On the left, a K-band AO-corrected image of the binary star $\alpha$-Ophiucus, observed from the Palomar Telescope. On the right, a set of spectrally dispersed two-telescope fringes produced by the instrument AMBER at the focus of VLTI. Both images are dominated by diffraction features such as fringes and rings and can also be affected by other artifacts like ghosts.

\section{Images in astronomy}

Images are the starting point of a lot of astronomical investigations. Even to the non-expert, because the image is a direct extension of one's intimate sense of sight, it offers rapid insights into complex situations. The image is the place where an observer will (1) identify new sources, (2) measure their position and brightness relative to a set of references and (3) follow their evolution as a function of time, wavelength and polarization. From these fundamental measurements, populating a multi-dimensional map $I(\alpha, \delta, \lambda, P, t)$ function of position, wavelength, polarization and time, an astronomer will improve his/her understanding (i.e. build a model) of a given object or event, that tells the story of an open star cluster, a group of galaxies, or that of a young planetary system, forming in the vicinity of a nearby star. The fair and efficient interpretation of images is essential to a wide range of scientific applications.

$\mathrm{Be}$ it in an actual imaging instrument, a spectrograph or an interferometer, the image is first and foremost, a peculiar optical locus, where the photons coming from a wide number of sources, and more or less uniformly distributed over the collecting surface (the pupil) of one or more telescopes, find themselves optimally segregated by geometric optics. It is possible to describe the result of this photon segregation process as the result $\mathbf{I}$ of a convolution product between two parts: one that is representative of the true distribution of intensities describing the source noted $\mathbf{O}$; and one that describes the instrumental response, that includes the properties of the atmosphere, the telescope and all the optics encountered by the light before reaching the detector. This instrumental response, called point spread function is noted PSF, such that: 


$$
\mathbf{I}=\mathbf{O} \otimes \mathbf{P S F},
$$

where $\otimes$ represents the convolution operation.

Much effort is devoted by telescope and instrument designers to reduce the impact of the instrumental contribution on the end product. For a great deal of astrophysical observations, the improvement is such that one can directly identify the object $\mathbf{O}$ to the image $\mathbf{I}$ without really paying attention to the PSF. The photon segregation process occuring in the image plane is however fundamentally limited by the phenomenon of diffraction. The scaling parameter that rules this limitation is the ratio between the wavelength of observation $\lambda$ and the characteristic dimension of the aperture used to perform the observation (the diameter of a single telescope, or the length of the interferometric baseline). To quickly estimate the angular resolution provided by a telescope, the following quick formula often comes in handy:

$$
\theta \approx 200 \times \frac{\lambda}{D}
$$

where $\theta$ is the angular resolution in milli-arcseconds (mas), $\lambda$ the wavelength in microns and $D$ the diameter of the aperture in meters. One can verify that a one-meter telescope observing in the visible $(\lambda=0.5 \mu \mathrm{m})$ offers a 100 mas $(0.1 ")$ angular resolution, and that an 8 -meter telescope observing in the near-infrared $(\lambda=1.6 \mu \mathrm{m})$ gets down to 40 mas.

Yet even in seemingly ideal observing conditions, the segregation of photons provided by the image is often not sufficient in solving some important problems such as: (1) the identification of faint sources or structures in the direct neighborhood of a bright object: in this context, the faint source one tries to detect is competing for the observer's attention with the diffraction features of its host or (2) the discrimination of sources of comparable brightness so close to each other that they are said non-resolved. Dealing with these two similar situations is the object of this presentation on diffraction-dominated observational astronomy.

\section{Coherence properties of light}

Electromagnetic radiation still contributes today to the great majority of the information collected by astronomical observatories that forms the basis of astrophysics: the properties of images produced by astronomical instrumentation can be described using the results of an early $\mathrm{XIX}^{\text {th }}$ century physics theory laid out by James Clerck Maxwell. Electromagnetic waves consist of synchronized oscillations of electric and magnetic fields that propagate through a medium at an actual velocity smaller or equal to $c$ (the speed of light through a vacuum). The electric and magnetic fields are orthogonal to one another so that one can specify the wave by keeping track of the electric field alone, which simplifies the description. Note that this presentation will not discuss polarization effects, a refinement that can be added later and won't change the results and properties derived. Electromagnetic (and therefore electric) waves, are solutions to Helmoltz's equation (also called the wave equation):

$$
\nabla^{2} \mathbf{E}-\frac{1}{c^{2}} \ddot{\mathbf{E}}=0,
$$

where $c$ represents the propagation speed of these waves, i.e. the speed of light. Natural solutions to this equation are oscillating functions with the following form:

$$
\mathrm{E}_{\nu}(t, x)=\mathrm{E}_{0} e^{i(k x-\omega t)}=\mathrm{E}_{0} e^{i 2 \pi(x / \lambda-v t)},
$$



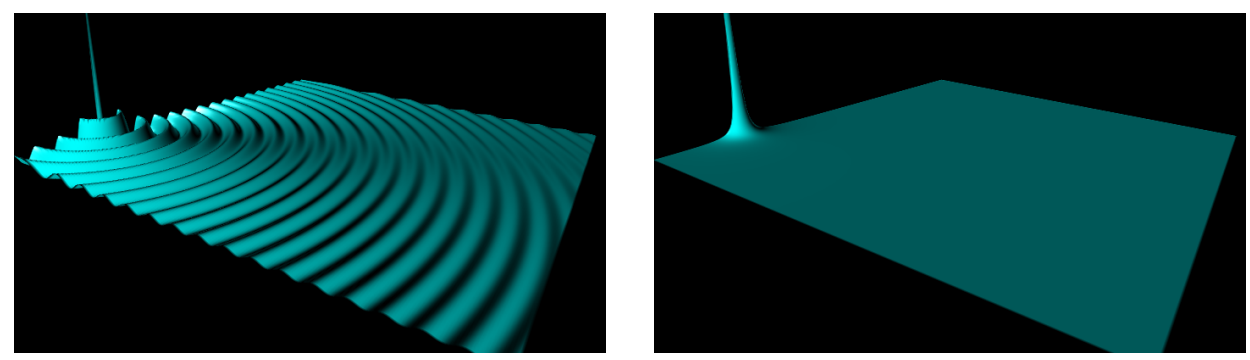

Figure 8. Propagation of a spherical wave. Left: imaginary instant snapshot of the complex amplitude of an electric field emitted by a point source. Directly observing this phenomenon would require a signal commuting time a million time shorter than state of the art fast switching semi-conductors can offer. Right: the static, time-averaged intensity associated to this same electric field, that can indeed be measured in the vicinity of a point source.

characterized by a frequency $v$ corresponding to the number of oscillations of the field per seconds (or Hertz) and the wavelength $\lambda$ that corresponds to the distance covered by the propagating electric field over the time of one oscillation. In a vacuum, these two quantities are related via the following inverse relation:

$$
\lambda=c / v \text {. }
$$

The complex exponential form of the oscillating solution of Eq. 4 allows to separate the time and space dependencies of the electric field. The spatial component is awarded a special name: the complex amplitude, noted $A(x)$ such that:

$$
\mathrm{E}_{v}(t, x)=\mathrm{A}(x) e^{-i 2 \pi v t} .
$$

While the complex amplitude is written as the function of a single variable $x$, one has to keep in mind that this complex amplitude is tri-dimensional. Thus if for instance, the origin of the electromagnetic field is a single point source, the electric field is a spherical function of a radius coordinate $r$ :

$$
\mathrm{E}_{\nu}(t, r)=(1 / r) \mathrm{E}_{0} e^{i(k r-\omega t)} .
$$

The applications covered in this text relate to what is referred-to as the optical: a regime of wavelength that covers the visible, going from $\lambda \sim 0.4 \mu \mathrm{m}$ to $\lambda \sim 0.8 \mu \mathrm{m}$ where our human eye is mostly sensitive and the infrared (IR) for wavelengths going up to $\lambda \sim 50 \mu \mathrm{m}$. Beyond the infrared, it is customary to use the frequency $v$ to describe the electromagnetic radiation. For wavelengths shorter than $\sim 100 \mathrm{~nm}$, it is customary to use the energy associated to the radiation. Taking $\lambda=1 \mu \mathrm{m}$ as a wavelength representative of the optical and converting it to a frequency:

$$
v=\frac{c}{\lambda}=\frac{3 \times 10^{8}}{10^{-6}}=3 \times 10^{14} \mathrm{~Hz}
$$

This really large number explains the specificity of the optical regime. The typical $\mathrm{read} /$ write access time of today's fast switching semi-conductors is of the order of $\sim 1 \mathrm{~ns}$. Which means that over the time it takes to switch at least once to take a snapshot, the electric field associated to optical light has oscillated more than $10^{5}$ times. Unlike what is possible in the radio, available readout electronics are not fast enough to record the value of the electric 

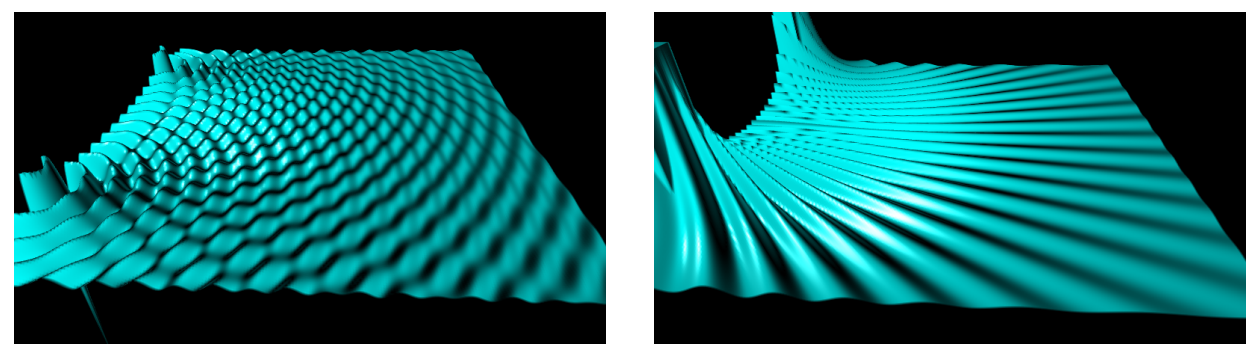

Figure 9. Visualisation of the interference phenomenon. Two point sources, located on the left hand side of the image at the same frequency, both emit a field propagating. Left: The two individual fields add up coherently and produce a rich wave pattern whose periodic properties depend on the frequency of the emission of the sources, and the distance that separates the two sources. Right: the static, time averaged intensity associated to this electric field. Unlike the single source scenario, in the far field (toward the right end of the image), intensity oscillations can be measured along the transverse direction.

field at any instant (see Figure 8). Instead, one measures the time averaged energy carried by the field and intercepted by a receiver, a quantity called the intensity:

$$
\begin{aligned}
I \propto\left\langle|\mathrm{E}|^{2}\right\rangle & =\int_{t_{0}}^{t_{0}+\tau} \mathrm{E}(t)^{2} \mathrm{~d} t \\
& =|\mathrm{A}|^{2}(\text { with } \tau>>1 / v) .
\end{aligned}
$$

that is proportional to the square modulus of the complex amplitude. In the absence of perturbations, the intensity recorded is a quantity that is only a function (see Figure 8) of the relative spacing between the source and the observer.

While apparently invisible when considering a single point source, the oscillating nature of the electric field becomes manifest when when a second source is present. The superposition principle states that the solution to this new situation is the sum of the two individual fields. Figure 9 shows what one such field looks like. We still don't have a receiver fast enough to be able to record the oscillations of the resulting field. The intensity associated to the field (visible in the right panel of Figure 9) however now also features some structure: the intensity oscillates and depending on where the receiver is placed, one can either record a maximum or a minimum of intensity. The distance between two consecutive maxima of intensity will be a function of the ratio between the wavelength $\lambda$ and the distance separating the two sources. Optical interferometry is primarily concerned with the characterization of these structures, refered to as interference fringes.

This mathematical description of the electromagnetic nature of light would suggest that interference phenomena such as the one that was just described should be commonplace. There are plenty of situations of every day life where the light of two or more sources overlaps on a surface and yet, fringes are a rare occurence. This is because our description has idealized the sources: the purely sinusoidal wave (Eq. 4) is only suited to the description of a laser beam.

The light emitted by thermal light sources like light bulbs or the hot gas of a star originates from a large number of semi-random spontaneous and therefore uncorrelated events like electronic transitions. A more accurate representation of such an emission process uses a series of wave-packets, such as the ones represented in Figure 10, which are a series of 


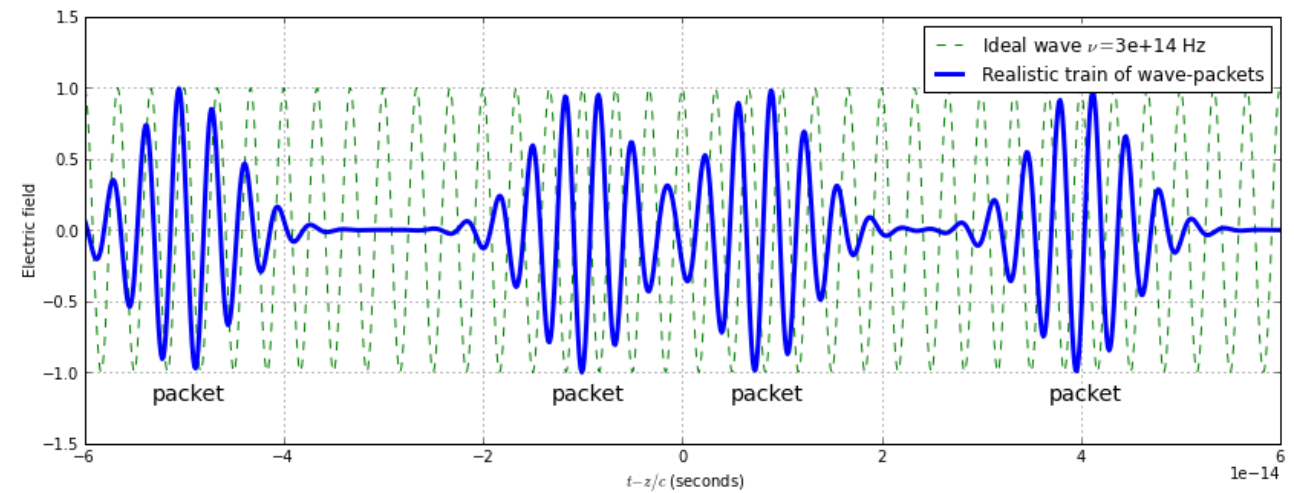

Figure 10. Improved, wave-packet based description of the electric field emitted by a thermal source (solid blue line) compared to the idealized sinusoidal field (dashed green line) used earlier. Random emission times and phase at the origin for each packet result in fluctuations in amplitude and phase of the electric field that will affect its capability to produce interferences.

damped oscillating fields $E_{k}$ modulated by an envelope function env and characterized by a random emission time $t_{k}$ and a random phase at origin $\Phi_{k}$ :

$$
\mathrm{E}_{\mathrm{k}}(r, t)=\operatorname{env}\left(t-t_{k}\right) \times e^{i 2 \pi\left(r / \lambda-v\left(t-t_{k}\right)+\Phi_{k}\right)} .
$$

The resulting electric field is no longer purely sinusoidal and fluctuates both in its amplitude and phase: Figure 10 compares this improved wave-packet model to the earlier ideal wave and shows that these fields are no longer synchronized, with the new electric field sometimes ahead of, and sometimes behind the reference. This desynchronization will affect the capacity of the light to produce interferences, a property characterized by a scalar (complex) quantity called the degree of coherence.

The degree of coherence is the result of time-averaged cross-correlation function. It can be used to compare and quantify how look-alike two distinct electric fields are, in which case it will be referred-to as the mutual coherence, or to compare one field with itself delayed in time, which will be referred-to as the self-coherence. This self-coherence is a normalized complex quantity:

$$
c(\tau)=\frac{<E^{*}(t) \times E(t+\tau)>}{<|E(t)|^{2}>},
$$

whose modulus $|c(\tau)| \leq 1$. In the case of the ideal wave model, the degree of self-coherence is always equal to one: regardless of the time delay, the electric field will always perfectly interfere with itself delayed in time.

In the wave-packed model, the field is only coherent with itself over when the delay is small. Figure 11 presents two scenarios: a small delay for which the original signal and its copy obviously correlate (ie. look alike); and a large delay (larger than the size of one fringe packet) for which the two fields clearly do not correlate anymore.

Nevertheless, even ni the second scenario, over a small range of time delays bound by $\tau_{0}$ (the coherence time), one can measure reasonably good correlation between the two signals. If one samples the same field twice, for instance by placing holes in a screen equally distant from a point source, and combines the two fields downstream such that their respective packets reach the same place within the coherence time, interferences can be observed. 

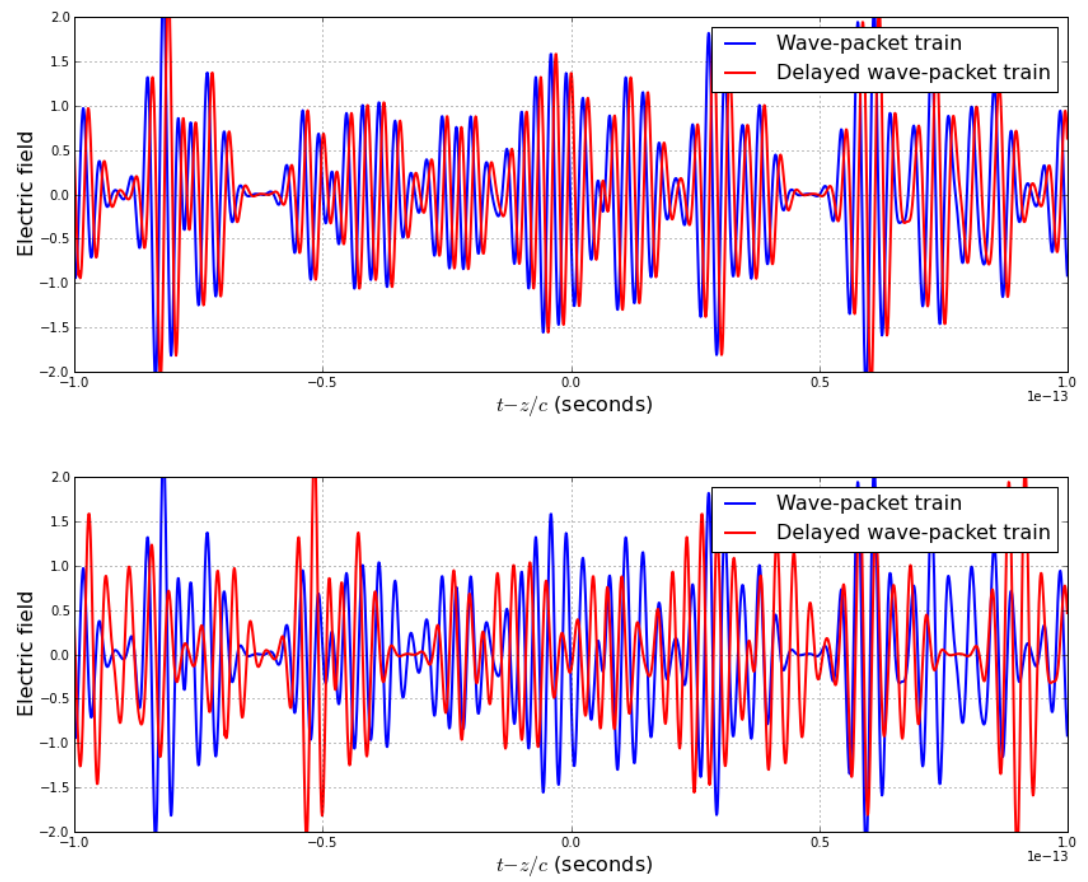

Figure 11. Illustration of the self-coherent properties of an electric field consisting of a collection of wave-packets. Top panel: when the time delay between the two is smaller than the coherence time $\tau_{0}$, the two fields do look alike and the result of their time-averaged cross-correlation exhibits a modulus $c \sim 1$. Bottom panel: beyond the coherence time, the two fields quickly decorrelate and the modulus of their self-coherence reaches $c \sim 0$.

When one considers two fields $E_{1}$ and $E_{2}$ emanating from different sources, one uses the degree of mutual coherence:

$$
c_{12}(\tau)=\frac{\left\langle E_{1}(t+\tau) E_{2}(t)^{*}\right\rangle}{\sqrt{\left\langle\left|E_{1}(t)\right|^{2}\right\rangle\left\langle\left|E_{2}(t)\right|^{2}\right\rangle}}=\frac{1}{\sqrt{I_{1} I_{2}}} \int_{\Delta t} E_{1}(t+\tau) E_{2}^{*}(t) \mathrm{d} t
$$

to quantify their capacity to interfere with one another. At this point, the reader may have already guessed that two electric fields originating from two distinct series of semi-random events have no chance of being correlated: the expected mutual degree of coherence is equal to zero.

These two elementary observations on the self- and the mutual-coherence of the electromagnetic fields emanating from thermal sources explain all the properties of the formation of image and interference fringes in astronomical instrumentation. Figure 12 offers a graphical summary of these two properties, and leads to the formulation of two simple but very important facts about the light of natural light sources:

- Fact \#1: the light emitted by one point source, collected by two or more apertures (or parts of one aperture), and recombined in a manner that all path lengths are equal, will lead to perfect interferences. Unresolved point sources are self-coherent. 
self-coherence

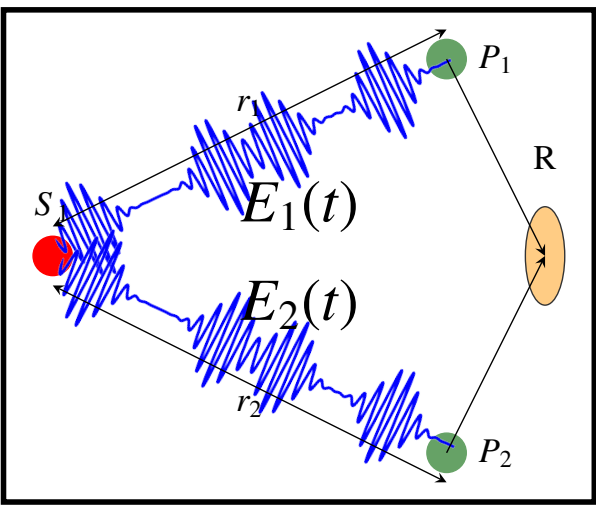

$S_{1}$ : the source

$E_{1}$ and $E_{2}$ : the electric fields

$P_{1}, P_{2}$ : the observing stations

$R$ : mono-pixel quadratic detector spatial incoherence

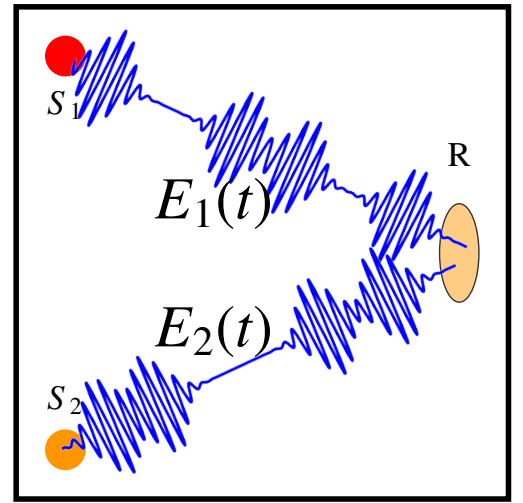

$S_{1}$ and $S_{2}$ : the two sources

$E_{1}$ and $E_{2}$ : the electric fields

$R$ : mono-pixel quadratic detector

Figure 12. Self- and spatial-coherence properties of the light emitted by astrophysical sources. Left: The light emitted by one point source, collected by two or more apertures, and recombined in a manner that guarantees that all path lengths are equal (from the source to the detector), will lead to perfect interferences: point sources are self-coherent. Right: The light from distinct astronomical sources, be it two distinct objects or two parts of the surface of one object, does not interfere: astronomical sources are spatially incoherent.

- Fact \#2: the light from distinct astronomical sources, either distinct objects or two parts of the surface of one object, does not interfere. Astronomical sources are spatially incoherent.

Most of the observing scenarios we are interested in in this lecture focus on a bright, unresolved object that, in most cases, can be treated like a bright point source, surrounded by fainter structures, such as planetary companions, a dust shell or elements of a circumstellar disk.

In such a situation, the effective resulting coherence will be dominated by the coherence properties of the bright source, but will be reduced due to the presence of faint sources whose light does not interfere with that of the bright source. The light of a single point source is perfectly coherent: in the case of an interferometer, the estimator of coherence, called the fringe visibility (or the visibility squared), is also equal to one; in the case of a single telescope observation, the image consists of a single, crisp PSF. The presence of additional structures around the bright point source will reduce the apparent visibility of the fringes (in the case of the interferometer) and/or make the single telescope image look fuzzier than on the point source alone: the effective coherence of one such extended source takes intermediate values between 0 and 1 .

Being able to measure the coherence of a source from an interferogram or an image assumes that one perfectly knows what the PSF or the fringe pattern acquired on a point source actually looks like. It turns out that several instrumental and environmental effects like the spectral bandpass, atmospheric dispersion, residual aberrations or drifts can result in an apparent loss of coherence. The task is somewhat easier when interpreting a two- 


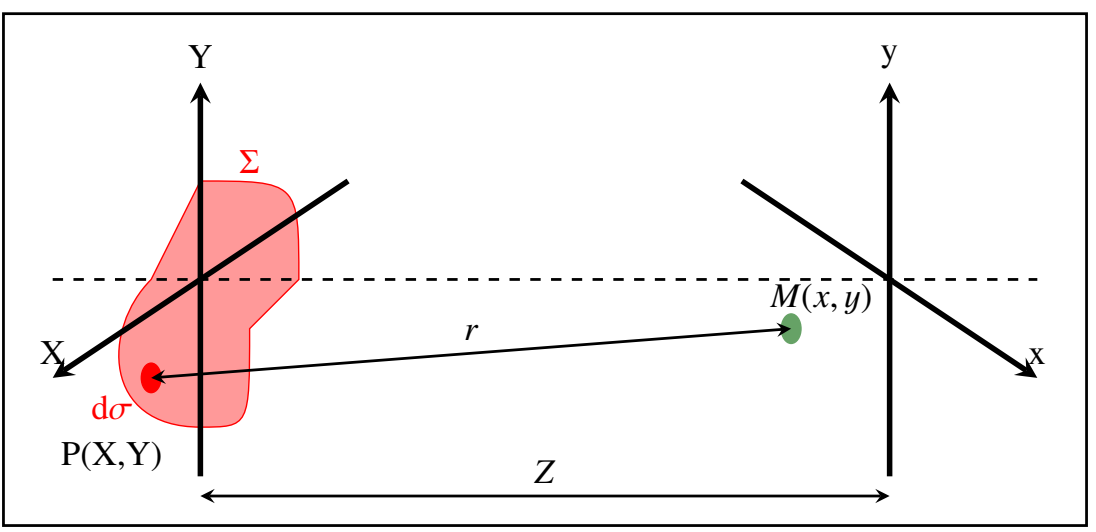

Figure 13. Schematic representation of the phenomenon of diffraction. A diaphragm described by the support $\Sigma$, on the left-hand side is uniformly lit by a point source at infinity. The symbols and notations present in this figure are used to determine the distribution of complex amplitude $A(x, y)$ in the right hand side plane located at a distance $Z$ from the diaphragm.

aperture interferogram, since the interferometer is really designed to produce unambiguous measurements of coherence, than from an image that contains a complex mix of overlapping spatial frequencies. The deconvolution of images, that is the inversion of Eq. 1, is in practice difficult when the PSF is not perfectly known as the problem is degenerate. We will get back to this very question toward the end of this presentation and see how we can addressit and make our coherence estimates unambiguous.

\section{Diffraction-dominated imaging}

Since another lecture specifically deals with interferometry, the discussion will from now focus on the properties of images produced by single telescopes. Hopefully, the reader will realize that it doesn't take long to adapt the following discussion to the case of a multiaperture interferometer.

Figure 13 introduces the symbols and the scenario used to describe the phenomenon of diffraction: on the left, a diaphragm or arbitrary shape described by the surface $\Sigma$, uniformly lit by a point source located so far toward the left that (under perfect observing conditions) the complex amplitude of the associated electric field is constant across the aperture. $\Sigma$ describes the aperture of the telescope used to do imaging. If one were to consider looking into interferometry from here, one would just have to split $\Sigma$ into a collection of sub-apertures.

The important relation to establish is one that relates the electric field (at least its complex amplitude) across the aperture $\Sigma$ to its counterpart projected on a screen located at a distance $\mathrm{Z}$ from the diaphragm. One elementary surface element $\mathrm{d} \sigma$ is singled out on this picture. This elementary surface element is the origin of a new spherical wave (a principle described by Augustin Fresnel in 1818). For a point $\mathrm{M}$ of coordinates $(x, y)$ located at a distance $\mathrm{r}$ from the origin of the wave, the contribution for the wavelength $\lambda$ to the local complex amplitude from $\mathrm{d} \sigma$ is given by:

$$
\mathrm{d} A(x, y)=\frac{1}{r} \times K \times A(X, Y) \times e^{j 2 \pi r / \lambda} \mathrm{d} \sigma,
$$


where $\mathrm{K}$ is a constant. Since we've established that the light associated to a single point source is coherent, we can write that the total electric field in right-hand side plane of Figure 13 is the result of a sum of emissions from all elementary point sources:

$$
A(x, y)=K \iint_{\Sigma} \frac{1}{r} \times A(X, Y) \times e^{j 2 \pi r / \lambda} \mathrm{d} \sigma .
$$

If the distance $\mathrm{Z}$ between the two diaphragms and the backend screen is sufficiently large in comparison to the dimension of the diaphragm, the distance $r$ can be approximated:

$$
\begin{aligned}
r & =\sqrt{Z^{2}+(X-x)^{2}+(Y-y)^{2}} \\
& \approx Z\left(1+0.5\left(\frac{X-x}{Z}\right)^{2}+0.5\left(\frac{Y-y}{Z}\right)^{2}\right) .
\end{aligned}
$$

So that the expression for the complex amplitude in the plane on the right hand side of Figure 13 can be rewritten as the result of:

$$
A(x, y)=\frac{K}{Z} e^{i 2 \pi Z / \lambda} \iint_{\Sigma} A(X, Y) \exp \left(\frac{i \pi}{\lambda Z}\left((X-x)^{2}+(Y-y)^{2}\right)\right) \mathrm{d} \sigma .
$$

This form of integral is called the Fresnel Transform. It is a non-linear transform whose computation can therefore be a bit cumbersome. It is however very general and can be used to compute the diffraction by a diaphragm for a wide range of situations. The Fresnel Transform of Eq. 18 can however be further simplified when the distance $Z$ between the diaphragm and the screen becomes very large, compared to the dimensions of the aperture:

$$
\exp \left(\frac{i \pi}{\lambda Z}(X-x)^{2}\right) \approx \exp \left(\frac{i \pi}{\lambda Z} x^{2}\right) \times \exp \left(\frac{-i 2 \pi}{\lambda Z} x X\right)
$$

when $\frac{X^{2}}{\lambda Z}<<1$. This situation is referred-to as the far field or the Fraunhofer diffraction. While it seems like an approximation, it is perfectly suited to the description of what is happening when a powered optics (see Figure 14) is used to conjugate an object, located at infinity, to its image, placed at a finite distance. In the focal plane of a telescope, the far field approximation becomes perfectly valid. The Fresnel Transform of Eq. 18 can be rewritten as:

$$
A(x, y)=K^{\prime} \iint_{\Sigma} A(X, Y) \exp \left(\frac{-i 2 \pi}{\lambda F}(x X+y Y)\right) \mathrm{d} \sigma .
$$

where the distance $Z$ has been replaced by the focal length $F$ of the imaging optics. It is convenient to express the coordinates in the image in terms of angular distances relative to the pointing axis, replacing the ratio $x / F$ and $y / F$ by angular coordinates $\alpha, \beta$. One can drop the $K^{\prime}$ constant as well to simplify the notations and just ensure in the computation that the total number of photons collected during an integration, is preserved by the transformation:

$$
A(\alpha, \beta)=\iint_{\Sigma} A(X, Y) \exp \left(\frac{-i 2 \pi}{\lambda}(\alpha X+\beta Y)\right) \mathrm{d} X \mathrm{~d} Y .
$$

which you may recognize as the two dimensional Fourier Transform of the distribution of the complex amplitude in the diffracting aperture. Unlike the Fresnel Transform, the Fourier Transform (hereafter represented by the symbol $\mathcal{F}$ is a linear operation that can be computed in an efficient manner. This is the form we will mostly use for the rest of the cases described in this lecture. 


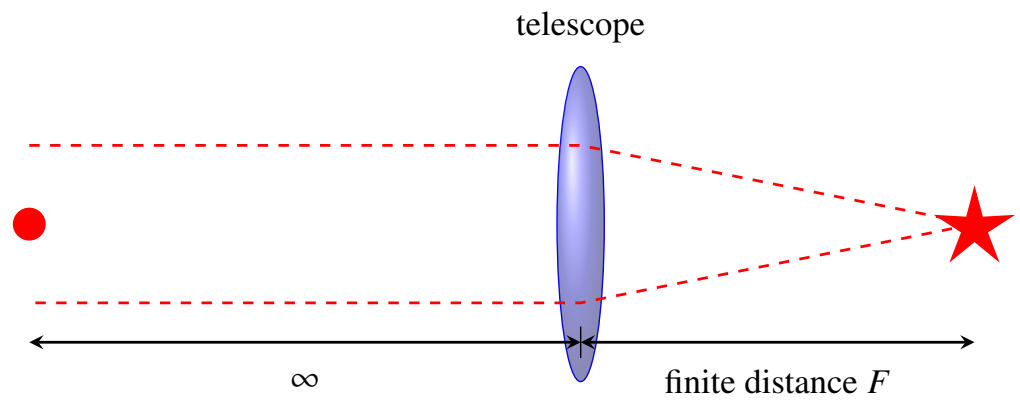

Figure 14. The impact of geometric optics on diffraction: a powered optical element conjugates an object located at infinity to an image at a finite distance in the focal plane. To compute the effect of the diffraction by the telescope in the focal plane, one can safely use the Fraunhofer diffraction relying on the computation of Fourier Transforms. To compute the effect of diffraction at any plane located in between the image and the diffractive aperture, one must use the more general Fresnel diffraction.

Equipped with this quantitative description of the diffraction and our previous observations on the coherent properties of astronomical sources, we can outline a recipe for the formation of an image:

1. an extended source can be described as a finite discrete collection of self-coherent point sources. The object function can be written as $O=\sum_{k} O_{k}$.

2. each point source uniformly illuminates the diffractive aperture. On axis, the complex amplitude $\left(A_{p}\right)$ is constant. The complex amplitude of each off-axis source includes a phase slope that is proportional to how far off-axis that source is.

3. because each point source is perfectly self-coherent, in the focal plane, the complex amplitude $A_{f, k}$ associated to each point source is the result of the Fourier Transform of the complex amplitude of the field intercepted by the aperture: $A_{f, k}=\mathcal{F}\left(A_{p, k}\right)$.

4. a detector only records the intensity associated to this point source: $I_{k}=\left|\mathcal{F}\left(A_{p, k}\right)\right|^{2}$. The effect of the phase slope associated to the off-axis source of index $k$ translates the resulting intensity pattern.

5. due to the spatial incoherence property of astronomical sources, the intensity patterns of all point sources add up: $I=\sum_{k} I_{k}$.

Since the light associated to each source is intercepted by the same aperture, the shape of the intensity pattern associated to each source (i.e. the PSF) is the same: the PSF is translation-invariant ${ }^{1}$. It is only modulated by the brightness of individual sources that acts as a scaling factor. The image can therefore be formally described as the weighted sum of PSFs. Figure 15 illustrates this property, which was given as early as Eq. 1 in this presentation but that we can now explain as the direct consequence of the coherence properties of astronomical sources.

Given the importance of the PSF in the shaping of the final image (see Figure 15), we need to see how the shape and size of the aperture, also known as the pupil, will impact

\footnotetext{
${ }^{1}$ When a single diffractive element is present only. In practice, the atmosphere, the relay optics inside the telescope and the instrument can render the PSF no-longer translation invariant. Over the small field of view we are dealing with here, these subtleties can be neglected.
} 


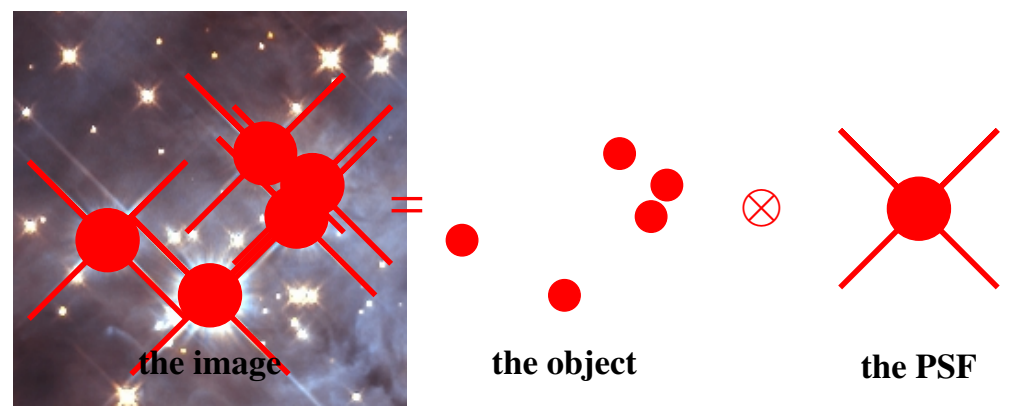

Figure 15. The image-object convolution relation illustrated: it is because of the spatial incoherence properties of astronomical sources present in the field (here extracted from a HST/NICMOS image of the Trapezium), that the image can be written as the result of a convolution product between the object function $O$ and the PSF of the telescope and its instrument.

the PSF. The theory of diffraction outlined earlier showed that the PSF can conveniently be computed as the result of the square modulus of the Fourier Transform of the illumination of the pupil:

$$
\operatorname{PSF}=\mid\left.\mathcal{F}(\text { pupil })\right|^{2}
$$

Real telescopes unfortunately have fairly complex pupils, featuring at least a central obstruction induced by the presence of a secondary mirror and spider vanes that give support to this secondary mirror. The primary mirror itself can also be made of several segments whose edges induce further diffraction. The PSF of a circular unobstructed telescope (known as the Airy function), only relevant for on-axis refractive telescopes or for an off-axis reflective one, is a useful reference to compare a real telescope to. The circular aperture is one of the few geometries for which the PSF has an analytical expression. Its radial profile is described by:

$$
\operatorname{Airy}(r)=4 \times\left|\frac{J_{1}(\pi r)}{\pi r}\right|^{2}
$$

where $r$ is the angular distance expressed in units of the ratio between the wavelength and the diameter of the aperture $(\lambda / D)$ and $J_{1}$ a Bessel function. This Airy pattern, represented in Figure 17 (using a logarithmic scale) features diffraction rings that extend very far away from its core. The Airy function meets its first zero for $r=1.22 \lambda / D$, which is often used to estimate the order of magnitude for the angular resolution of an observing setup. Regardless of the details of the aperture, the ratio $\lambda / D$, where $D$ is the characteristic dimension of the diffractive system ${ }^{2}$, will always be the right order of magnitude to consider to characterize the angular resolution of an optical setup. For an 8-meter diameter telescope operating in the near infrared, the ratio $\lambda / D$ is of the order of $\sim 10^{-7}$ radians. Such a small value makes the radian a inconvenient unit to manipulate. In practice, instrument plate scales for imagers at the focus of space-borne or ground based AO-corrected telescopes are usually expressed in milli-arc seconds per pixel. The conversion from radians to arcseconds given by:

\footnotetext{
${ }^{2}$ It can be the diameter of a single telescope or the distance separating two sub-apertures when dealing with interferometry.
} 


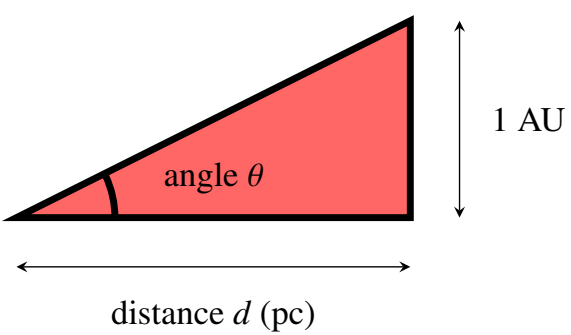

Figure 16. The parsec is the distance at which a projected distance of $1 \mathrm{AU}$ (astronomical unit) corresponds to an angular distance of one arc second. One parsec is therefore roughly equal to $2 \times 10^{5} \mathrm{AU}$ (see text).

$$
\begin{aligned}
\theta\left[{ }^{\prime \prime}\right] & =\frac{180 \times 3600}{\pi} \times \theta[\mathrm{rad}] \\
& \simeq 206264.8 \times \theta[\mathrm{rad}]
\end{aligned}
$$

gave us the short-hand formula of Eq. 2 for the angular resolution in mas. The 206,264.8 conversion factor (often rounded to $2 \times 10^{5}$ ) is an order of magnitude that is good to keep in mind. It is indeed the scaling factor between phenomena occuring inside a planetary system (where distances are measured in astronomical units or AU) and phenomena occuring over interstellar distances (for which distances are measured in parsecs). Since the parsec was defined as the distance at which a projected distance of $1 \mathrm{AU}$ corresponds to an angle of one arc second (see Fig. 16):

$$
\begin{aligned}
\tan 1^{\prime \prime} \sim 1^{\prime \prime} & =1 \mathrm{AU} / 1 \mathrm{pc} \\
\theta\left[^{\prime \prime}\right] & =1 / d[\mathrm{pc}] \\
1 \mathrm{pc} & =206264.8 \mathrm{AU} .
\end{aligned}
$$

\section{High-contrast imaging}

From the rather large sample of extrasolar planets known at the time of this writing, only a dozen systems featuring planetary candidates have been imaged by space-borne and groundbased telescopes. Why is this task so difficult?

For a nearby planetary system, i.e. located $\sim 20$ parsecs away from our own Solar system, planets on orbital distances between 1 and $10 \mathrm{AU}$ will appear at angular separations ranging from 50 mas to $0.5^{\prime \prime}$ which seems to be within the angular resolution reach of modern telescopes, even when observing in the near-infrared. The difficulty in the direct imaging of extrasolar planets lies in the very large difference of luminosity between a faint planet and its bright host star. The brightness ratio, also known as the contrast ratio, of a mature Earth-like planet in a $1 \mathrm{AU}$ orbit around an equally mature Sun-like star would be characterized by an incredibly large $10^{-10}$ contrast ratio. A more favorable scenario is that of a self-luminous giant planet like Jupiter in orbit around a young star for which the contrast ratio could stay as high as $10^{-6}$ for a few million years. The right panel of Figure 17 illustrates the difficulty of the situation, by comparing these two scenarios to the ideal PSF profile of a circular aperture. Even at the largest plotted angular separation $(10 \lambda / D)$, the signal one would like to detect 

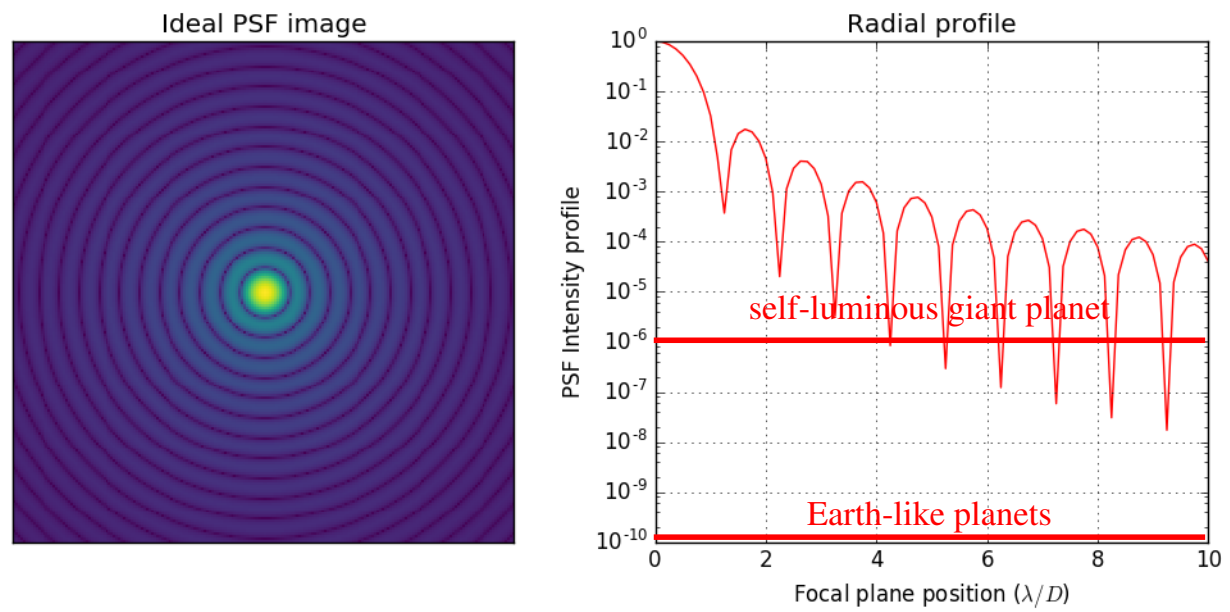

Figure 17. Left: 2D representation of a the perfect point spread function (PSF) of a circular aperture (using a non-linear color scale). Right: radial profile of the same PSF, over a $10 \lambda / D$ range of angular separation. Two horizontal lines mark the expected relative brightness (contrast) of two types of planets: self-luminous Jupiter-like at the $10^{-6}$ level and reflective Earth-like at the $10^{-10}$ level. Both lines lay several orders of magnitude below the PSF.

is still orders of magnitude fainter than the photon noise of the local diffraction structures of the on-axis bright star. When the pupil of the telescope features additional structures such as a central obstruction and spider vanes, the situation is even less favorable.

Simply masking out the PSF in the focal plane does not contribute much: it can help avoid saturation problems on the brightest parts of the PSF but the photon noise of the light present in the diffraction rings will still be the dominant source of noise. To facilitate the detection of faint structures present in the neighborhood of a bright object, one needs to reduce overall on-axis transmission so as to reduce the bright object's photon noise. The need for high-contrast imaging gave birth to a wide number of techniques amongst which two major families emerge: apodization and coronagraphy. Since the early 2000s, this still active area of research has generated a lot of enthusiasm and become extremely sophisticated. The goal of this presentation is not to give the readers a detailed description of the state of the art, but rather to introduce the important ideas that will help understand how the challenge can be addressed. This will require the application of the diffraction theory that was described earlier.

\subsection{Pupil apodization}

We know that the properties of the PSF of a diffractive aperture are directly related to the Fourier transform of the illumination of that aperture. The diffraction rings observed in the PSF of a circular telescope can be attributed to the sharp transmission edge of the pupil. By tuning the transmission profile of the aperture, one can expect to be able to alter the PSF and its diffraction features. This procedure is referred-to as an apodization ${ }^{3}$ and it can result in a PSF featuring no diffraction rings. Figure 18 shows how this apodization effect

\footnotetext{
${ }^{3}$ apodization litterally refers to the process of removing something's (or someone's!) foot
} 

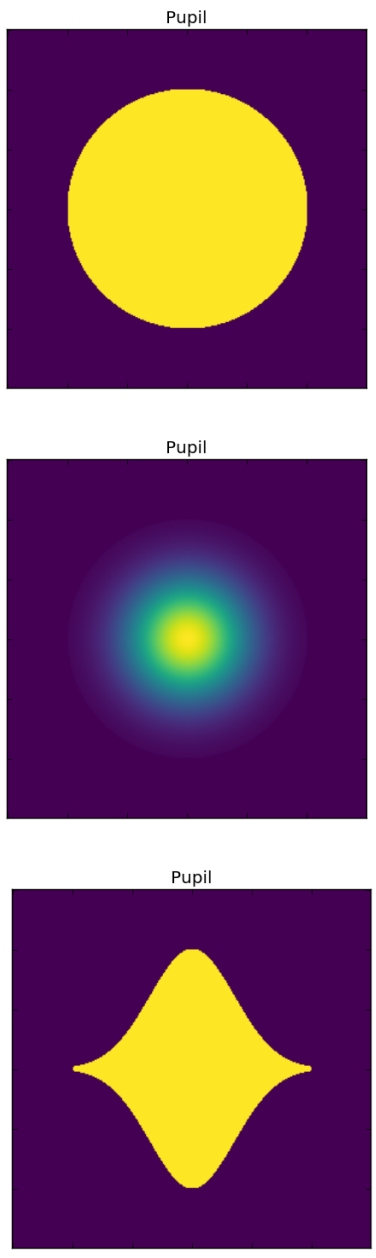

PSF

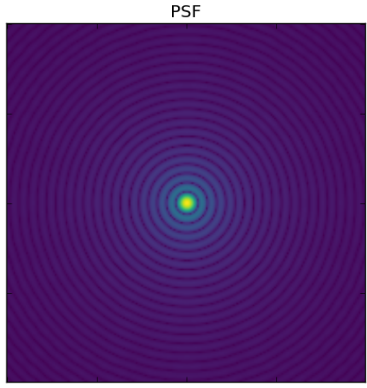

PSF

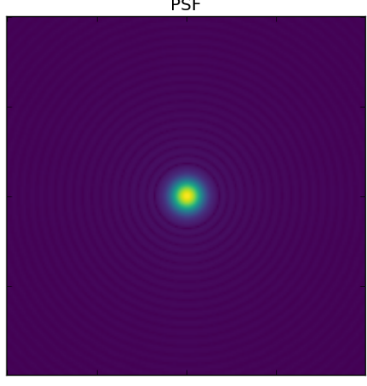

PSF

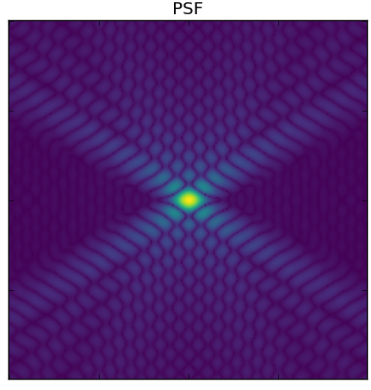

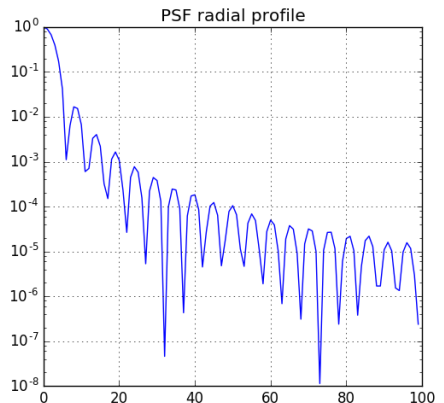
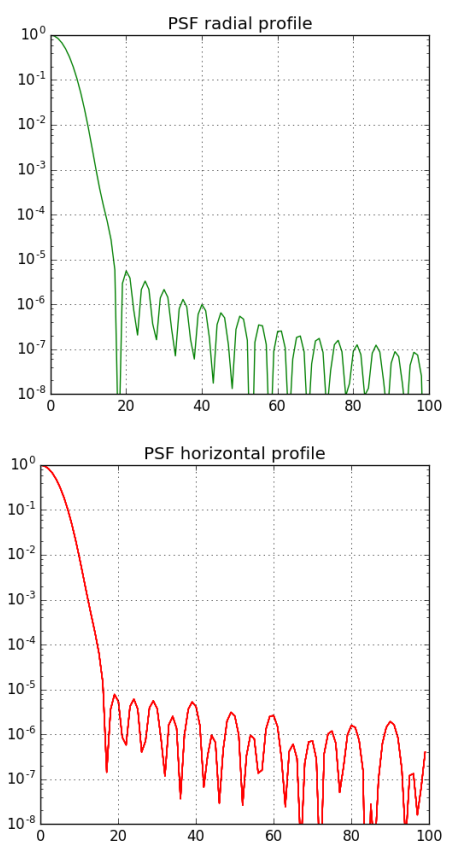

Figure 18. Pupil apodization: the diffraction features of the unobstructed circular aperture (top row) are compared to those produced by two apodization techniques, using either a variable transmission radial mask (middle row) or a binary wave-shaped mask (bottom row). The throughput is reduced from 100 $\%$ to $\sim 25 \%$ for the continuous apodization presented in the middle row and to $\sim 56 \%$ for the binary apodization presented in the bottom row. In both cases, the original PSF (top row) is replaced by a PSF better suited to the detection of high-contrast companions. The PSF profiles visible in the right-hand column of panels, using a logarithmic scale, reveal that both apodization approaches can produce PSFs with diffraction features with a contrast that rapidly drops below $10^{-5}$ offering a high-contrast detection advantage over the full aperture for angular separations as low as three resolution elements $(\sim 3 \lambda / D)$.

can be achieved using a fairly simple shaped-pupil mask placed over the original aperture of the telescope. At the cost of some throughput (corresponding to the original aperture surface now covered by the apodization mask), and some angular resolution (the effective aperture size shrinks because of the mask), the PSF features two symmetric dark regions at an orientation that can be adjusted by rotating the apodization mask. The comparison of the PSF profiles represented along the horizontal axis for both apertures shows that the apodization contributes to reducing the brightness of the diffraction by more than two orders 


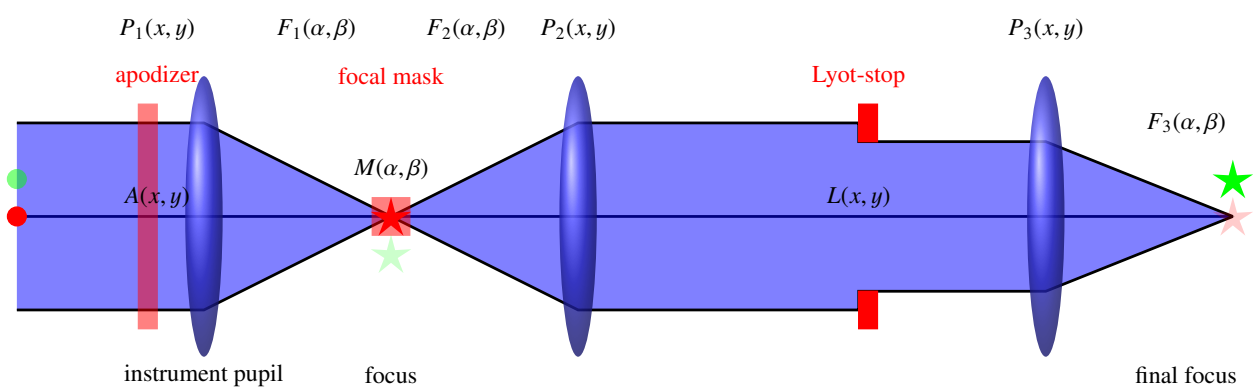

Figure 19. Schematic representation of a coronagraph. The light enters from the left hand side of the diagram and the final (high-contrast) image plane is on the right hand side. The important components of the coronagraph are highlighted in red. From left to right: the apodizer, the focal plane and the Lyotstop, placed in a relayed pupil. The relay optics (represented by lenses in this diagram) located after the first focus (where the focal plane mask is inserted) are required to form a conjugate pupil plane. The final lense is an imaging optics that produces the final high-contrast image with the appropriate f ratio.

of magnitude. The energy previously present in the diffraction rings now contributes to a wider PSF core, of radius $\sim 3 \lambda / D$. The size of the new PSF core defines what is now often referred-to as the inner working angle (IWA) of the high-contrast imaging system.

The solution presented here is by no means optimal: the apodization profile chosen to produce these figures follow more or less gaussian shapes. In the litterature, a special class of functions called spheroidal prolates $[1,2]$ features properties that make them ideal for highcontrast imaging, able to deliver theoretical contrast ratios five orders of magnitude better than those presented in Figure 18. Because the properties of the apodized PSF only depend on the modified pupil shape, the apodized PSF is not more wavelength dependent that its non-apodized counterpart (see the $1 / \lambda$ scaling factor in Eq. 21 ), and will be weakly sensitive to pointing errors. Implemented as it was just described, it however results in throughput and angular resolution loss as it reduces both the effective collective surface area and the effective diameter of the aperture.

Apodization can be achieved using as suggested above, with an aperture mask that suppresses part of the original aperture, or by redistribution of the light which preserves preserves both throughput and resolution [3]. The price to pay for one such remapping of the aperture is a PSF that is no longer position invariant (for which the image - object convolution relation of Eq. 1 is no longer valid), at least until that remapping can be undone [4].

To bring its full benefit, the apodization must be adapted to the features of the aperture [5]: the presence of a central obstruction and spider vanes in the pupil would render the simple solutions provided in Fig. 18 useless. The high-performance apodization of real life telescopes is in practice a complex optimization problem requiring a trade-off between IWA, overall transmission, and extinction.

\subsection{Coronagraphy}

Whereas apodization aims at shaping the PSF so as to reduce the impact of the diffraction rings and spikes, coronagraphy aims at suppressing the light of a bright source from the focal plane. The technique is slightly more complex than straightforward apodization as it requires intervention in at least two optical planes. Figure 19 provides a schematic representation of the elements constituing a coronagraph. Three elements are highlighted in red. Going from 
left to right, we have: the apodizer that was described earlier, the focal plane mask, located as its name aptly suggests, in the image plane and the so-called Lyot-stop, located in a optical plane that is conjugated with the entrance pupil, after the focal plane mask. While not a part of the original design of the coronagraph, the benefit of apodization described earlier also contributes to improving the coronagraph and both techniques are now used simultaneously [6].

The bulk of the light associated to the on-axis bright source (represented in Figure 19 by the left-hand side red dot) encounters the focal plane mask that can either occult it (by absorption or reflection), or dephase it. It was pointed out earlier that masking out the central part of the PSF alone does not result in a suppression of the diffraction features outside of this mask. But if one uses optics to relay the pupil (which is the role of the second lens in the diagram), the use of a second mask, the so called Lyot-stop, completes the effect of the focal plane mask and increases the contrast in the final focal plane. Since it misses the focal plane, the light of an off-axis source (represented by the green dot located next to the star) is almost entirely transmitted by the coronagraph and becomes visible in the final focal plane.

\subsection{Coronagraphic formalism}

Using our recently acquired diffraction computation skills, we can complete the schematic representation of the coronagraph with a formal description of what is happening at its different stages. Above the different elements represented in Figure 19, a few labels are provided that will be used and referred-to, to describe the complex amplitude of the electric field of starlight going through the coronagraph.

To better distinguish what is taking place in the pupil from what is happening in the focal plane, two sets of coordinates are used: linear $(x, y)$ position coordinates in the aperture and angular $(\alpha, \beta)$ coordinates in the image. The impact of the three elements of the coronagraph is described by the apodizing function $A(x, y)$, the focal plane mask function $M(\alpha, \beta)$ and the Lyot-stop function $L(x, y)$. We now know (see Eq. 21) that a Fourier transform $\mathcal{F}$ ) relates the complex amplitude in the pupil to the one in the focus: each time the optical system goes back and forth between pupil and image, a Fourier transform is at work. While cumulating the effect of consecutive Fourier transform may sound like a terrible idea at first, it turns out to be fairly simple since:

$$
\mathcal{F}(\mathcal{F}(f(x, y)))=f(-x,-y)=\mathcal{R}(f(x, y)),{ }^{4}
$$

where $\mathcal{R}$ represents the coordinate flip (or reverse) operator. As long as we don't land on a detector that records the square modulus of the complex amplitude (see Eq. 10), the elements of the coronagraph directly interact with the local complex amplitude. This interaction is modeled by a multiplication by a complex amplitude gain $g$, with a modulus $0<|g| \leq 1$ (these elements do not amplify the signal) and possibly a phasor $e^{i \phi}$ term if the component introduces a phase delay $\phi$. Another nice property of the Fourier transform that helps understand how the different components affect the final focal plane electric field (and ultimately the intensity), is the convolution property:

$$
\mathcal{F}(f(x, y) \times g(x, y))=\mathcal{F}(f(x, y)) \otimes \mathcal{F}(g(x, y)),
$$

that says that the Fourier transform of a product is equal to the convolution product of individual Fourier transforms. Thus since the impact of an element of the coronagraph is locally modeled by a multiplication, in the next plane, it results in a convolution.

\footnotetext{
${ }^{4}$ The flip of the $(x, y)$ coordinates observed after going from pupil to focus and then back to focus, reproduces the effect of a lens that produces an inverted image.
} 
With these elements in mind, we can finally describe formally what is taking place in the coronagraph, using the following sequence of operations:

- $P_{1}=A$

( $A$ and $P_{1}$ have the same support)

- $F_{1}=\mathcal{F}\left(P_{1}\right)$

(going to focus $\rightarrow$ Fourier transform)

- $F_{2}=M \times F_{1}$

(focal plane mask multiplies the complex amplitude)

- $P_{2}=\mathcal{F}\left(F_{2}\right)$ (going back to pupil plane $\rightarrow$ Fourier transform)

$$
P_{2}=\mathcal{F}\left(M \times F_{1}\right)
$$

(explicit $F_{2}$ )

$P_{2}=\mathcal{F}(M) \otimes \mathcal{F}\left(F_{1}\right)$

(convolution property)

$P_{2}=\mathcal{F}(M) \otimes \mathcal{R}\left(P_{1}\right) \quad\left(P_{2}=\right.$ input pupil convolved by the mask Fourier-transformed $)$

- $P_{3}=P_{2} \times L$

(the Lyot-stop blocks parts of the pupil)

- $F_{3}=\mathcal{F}\left(P_{3}\right)$

(going to final focal plane $\rightarrow$ Fourier Transform)

$$
\begin{aligned}
& F_{3}=\mathcal{F}\left(P_{2}\right) \otimes \mathcal{F}(L) \\
& F_{3}=\mathcal{R}\left(M \times \mathcal{F}\left(P_{1}\right)\right) \otimes \mathcal{F}(L)
\end{aligned}
$$

(convolution property)

(explicitation of terms)

- $I=\left|F_{3}\right|^{2}$

(intensity is square modulus of complex amplitude)

Figure 20 illustrates these different steps by representing the light of an on-axis (top row) and an off-axis (bottom row) source as it goes through the different planes of a coronagraph, using no apodization and a simple focal plane mask occulting the core of the PSF along with its first Airy ring (radius $\sim 2 \lambda / D$ ). The most important property to observe is the transition from the second to the third column: in the pupil plane that follows the focal plane mask, the light of the on-axis source is no longer uniformly distributed but tends to concentrate near the contours (sharp edges) of the input pupil that features here a central obstruction and spider vanes. To filter this light that would otherwise find its way back to the final focal plane, the Lyot-stop masks out these regions, resulting in a slightly undersized output pupil, with a larger central obstruction and thicker spider vanes. In the final focal plane, the light of this on-axis source is considerably attenuated.

The same operations can be applied to the electric field associated to an off-axis source. The off-axis position will result in a phase slope across the aperture. It it is sufficiently far off-axis (here $\geq \sim 2 \lambda / D$ ), the bulk of this electric field off-axis misses the focal plane. In the output pupil, the light of this source remains mostly uniformly distributed and the Lyot-stop only induces a reduction of the throughput. In the final focal plane, the light of this off-axis source is almost integrally transmitted: the on-axis attenuation combined with a good off-axis transmission results in images revealing faint structures in the bright star's neighborhood, that would otherwise remain invisible.

The coronagraph used to produce the images of Figure 20 uses an occulting mask, a configuration known as the classical Lyot-coronagraph [7] as it replicates (with a smaller occulting mask) the elements that enabled Bernard Lyot to reveal the corona of the Sun in the early 1930s. A review of the litterature will reveal the existence of a wide variety of coronagraphs that use different types of masks that can also induce phase differences $[8,9]$, include subwavelength gratings [10] and feature geometries that split the focus into quadrants $[11,12]$. The combination of the coronagraph with an apodizer [13] increases the number of possibilities.

\section{Atmospheric turbulence and Adaptive Optics}

The purpose of high-contrast imaging devices is to suppress from an image the on-axis static diffraction signature of an optical system that includes the telescope, the beam transfer and 

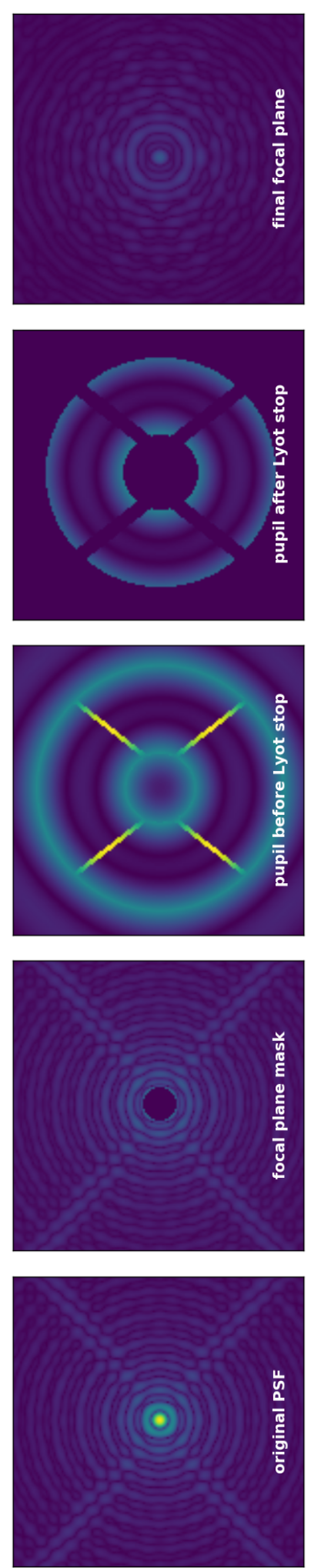
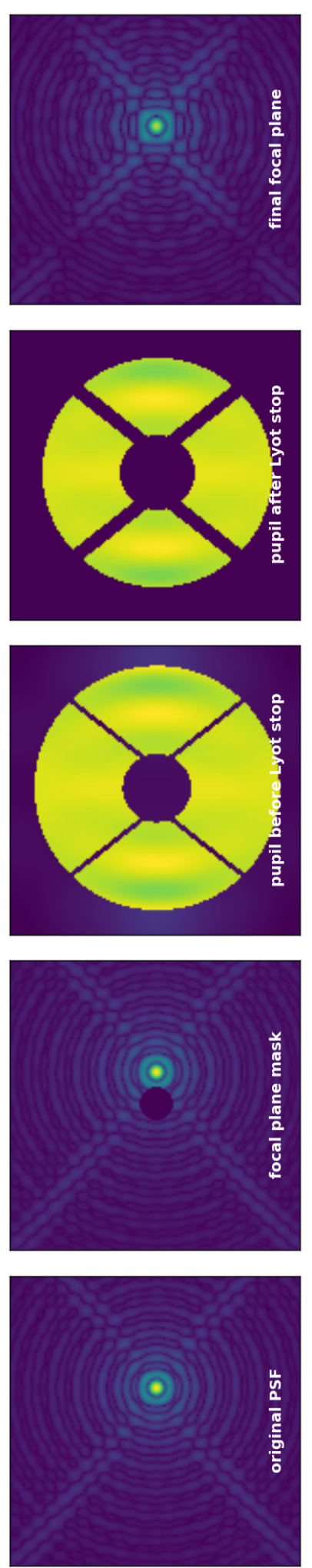

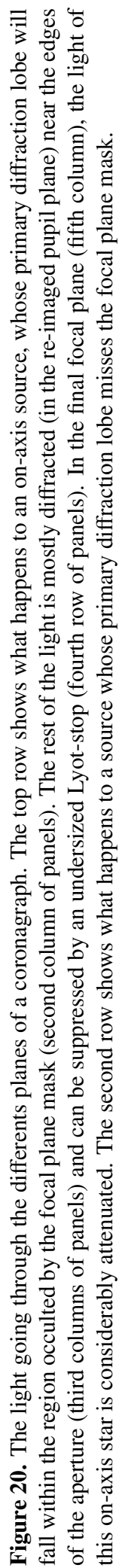


the instrument optics. The higher the design performance of the retained solution (often quantified by a level of contrast at a given separation), the more sensitive that solution ends up being to changes in the expected system configuration. One important optical element has however thus far not been taken into consideration: for ground based observations, the atmosphere ends up being a very important element that can quickly wreak havoc on the effective coronagraphic performance. One of the first descriptions of the effect of what we now call atmospheric turbulence can be found as early as 1704 in Isaac Newton's Opticks:

"If the Theory of making Telescopes could at length be fully brought into Practice, yet there would be certain Bounds beyond which Telescopes could not perform. For the Air through which we look upon the Stars, is in a perpetual Tremor [...] The only Remedy is a most serene and quiet Air, such as may perhaps be found on the tops of the highest Mountains above the grosser Clouds.” Book I, Prop. VIII, Prob. II.

The formation of images through a turbulent atmosphere is a complex process, so much that atmospheric optics is a research topic on its own. The three dimensional nature of the atmosphere results in multiple types of degradations: agitation of the image, spreading of the point spread function due to high-order wavefront aberrations and scintillation induced by high altitude turbulence resulting in intensity fluctuations. Figure 21 illustrates the typical effect of turbulence for a 1-meter diameter telescope observing in the visible: the original PSF on the left, with most of the light $(\sim 84 \%)$ concentrated over a $\sim 2 \lambda / D$ disk is replaced by a random speckle pattern that extends over a much larger area, suggesting the existence of smaller diffractive structures (the atmospheric turbulence cells) [14]. The typical dimension $r_{0}$ of these cells is called the Fried parameter [15] and the turbulence characteristic evolution time $t_{0}$ depends on the ratio between $r_{0}$ and the velocity $v$ of the turbulent layers. A good observing site is characterized by a large $r_{0}$, meaning that the turbulence is weak and a large $t_{0}$, meaning that it moves slowly. Typical turbulence properties for an average site in the visible $(\lambda=500 \mathrm{~nm})$ are:

- $r_{0} \sim 10 \mathrm{~cm}$

- $v \sim 10 \mathrm{~m} / \mathrm{s}$

- $t_{0} \sim 3 \mathrm{~ms}$

What this means is that the high angular resolution potential is no longer just set by the size of the aperture, but also by the properties of that turbulence. In the diffraction scenarios discussed so far, the distribution of complex amplitude for an on-axis source was assumed to be constant across the diffractive aperture: the wavefront was assumed to be perfectly flat. The atmospheric turbulence drastically alters this situation and introduces random phase delays that corrugate the wavefront (see the middle panel of Figure 21 for an example of phase screen).

The structure of the wavefront is not entirely random and is driven by thermodynamics [14]. One example of Kolmogorov phase screen is represented in the middle panel of Fig. 21. The variance between two parts of the wavefront separated by the distance $\rho$ :

$$
D_{\Phi}(\rho)=\left\langle\left|\Phi_{a}(r)-\Phi_{a}(r+\rho)\right|^{2}\right\rangle_{r}
$$

is a $2^{\text {nd }}$ order structure function characterized by one single parameter $r_{0}$ introduced earlier as Fried's parameter, so that:

$$
D_{\Phi}(\rho)=6.88\left(\frac{|\rho|}{r_{0}}\right)^{5 / 3} \text {. }
$$

The power spectrum of the phase deduced for a Kolmogorov phase screen [16, 17]: 

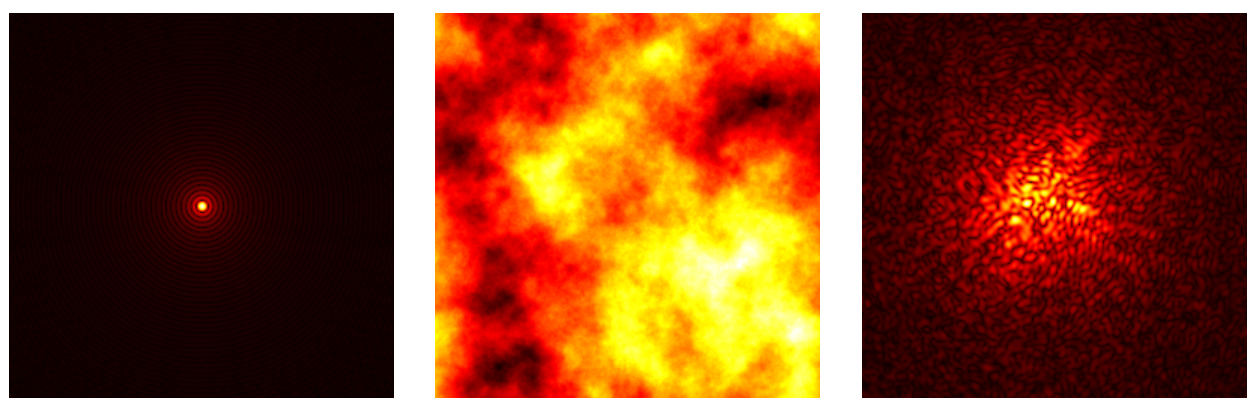

Figure 21. Left: theoretical Airy pattern produced by an unobstructed telescope. Middle: example of Kolmogorov phase screen induced by atmospheric turbulence. Right: instantaneous seeing-limited point spread function experienced when observing through one such atmospheric phase screen.

$$
W_{\Phi}(f)=<|\mathcal{F}(\Phi(\rho))|^{2}>=0.0228 r_{0}^{-5 / 3} f^{-11 / 3},
$$

shows that the distribution of phase follows a power law with a negative coefficient, which means that the atmosphere introduces more low order aberrations (associated to a low $f$ ) such as tip-tilt (pointing), focus, astigmatism and coma, than high spatial frequencies. The computation of the diffraction by the aperture (see Eq. 21) is still possible in the presence of turbulence, but the complex amplitude in the aperture $A(X, Y)$ must now include the atmosphericinduced phase delay $\Phi$, so that: $A(X, Y)=e^{i \Phi(X, Y)}$.

The impact of the Kolmogorov phase screen is visible on the right hand side panel of Figure 21 that features a short exposure image that keeps changing with a characteristic time $t_{0}$. One can see that while the PSF spreads out, it is still made of small structures called speckles whose characteristic size remains of the order of $\lambda / D$, suggesting that some highorder spatial frequency content can be recovered from the images if one is able to acquire them with an exposure time of the order of $t_{0}$. This is the object of speckle interferometry $[18,19]$ which won't be discussed here. A long exposure image through turbulence would wash out these speckles and result in an extended smooth PSF, characterized by a full width half-max of the order of $\lambda / r_{0}$.

Under such observing conditions, a high-contrast imaging device like a coronagraph, originally designed to take out the static component of the aberration, has very little chance of contributing to a contrast improvement in the image. The energy associated to the flux of the bright star, previously concentrated in the central diffraction feature $(\sim 84 \%)$ is now spread out over a wide number of fainter speckles. The same thing is also happening to the image of any other source in the field, resulting in an even lower chance of detecting any faint structure near the bright target. Corrective measures have to be taken to restore the wavefront entering the coronagraph and make it as flat as possible again.

This real-time compensation of the wavefront is the goal of the technique known as adaptive optics (AO). First described in the 1950s [20], and deployed by civilian astronomers in the early 1980s [21], AO is now a tool available at all major ground based observing facilities that exists in a wide variety of flavors: single or multi-conjugated, involving natural guide stars (NGS) or artificial (laser) guide stars (LGS). For the applications discussed here, AO is used in its simplest possible form: NGS - SCAO. Indeed, because it is focused on a very small field of view (of the order of one arc-second), high-contrast imaging requires singleconjugated adaptive optics (SCAO) and its targets, which are nearby stars, are bright enough to serve as the guide star for the adaptive optics. 


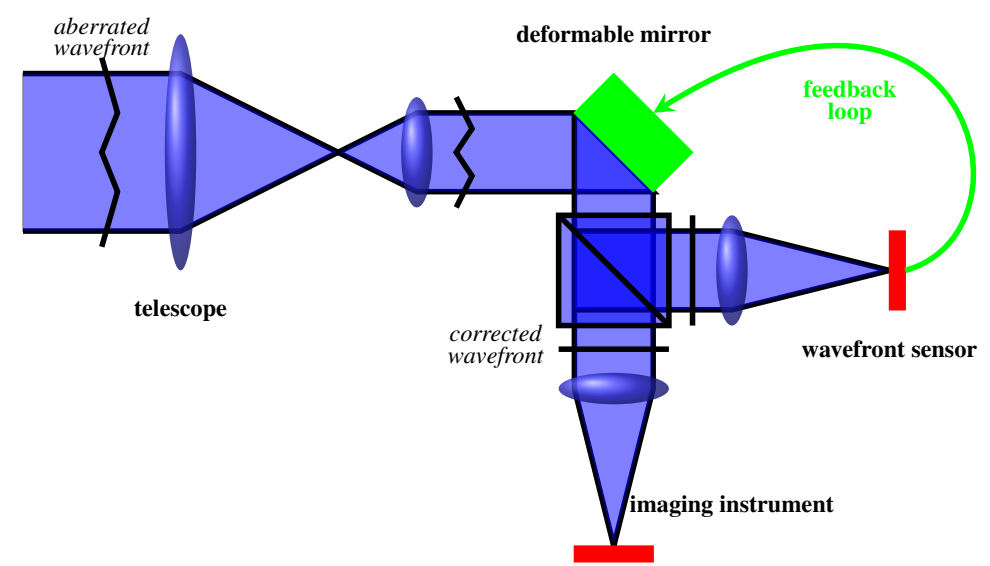

Figure 22. Schematic representation of the different elements of a closed-loop adaptive optics-fed imaging system. Starlight enters from the left-hand side of the diagram via the telescope, represented by a single lens. The impact of the atmospheric turbulence is represented by the $\mathrm{W}$-shaped wavefront, that propagates through the system. Relay optics make it possible to project the pupil of the telescope onto a deformable mirror (DM) whose shape can be adjusted to compensate the atmospheric effect on the current wavefront before it feeds the imaging instrument. To determine the shape the DM should take, some light is sampled before the imaging instrument and fed to the wavefront sensor (on the righthand side of the diagram). The analysis of the information collected by the wavefront sensor will drive a feedback loop that acts on the DM and results in an improved image.

An AO system requires two basic functionalities. The first is wavefront control, that is the ability to act on the wavefront of sources present inside the field of view, typically (but not only, as we will see later), to flatten it so as to improve image quality. The second is wavefront sensing, that is the ability to diagnose what is wrong with the current input wavefront, and to determine what can be done in order to correct for it. Figure 22 provides a schematic representation of how these two elements are combined to make up an AO system. It should be pointed out that wavefront sensing can take a wide variety of flavors such as the Shack-Hartmann, the curvature sensor [22] or the pyramid sensor [23]. The ideal wavefront sensor simultaneously combines good sensitivity, ie. the ability to operate on faint guide stars; linearity, ie. the ability to run an unambiguous diagnosis of the wavefront; and a large capture range, ie. the ability to operate in the presence of large or small wavefront errors. Real life sensors all seem to be able to only simultaneously gather two of these qualities at a time [24], which means that the choice of the wavefront sensor will have consequences on the final outcome. This topic will not be further discussed here and readers interested in this topic are invited to refer to textbooks dedicated to the topic of adaptive optics [25, 26]. We however need to take a closer look at the wavefront control to be able to understand some important features of AO-corrected images.

It was shown earlier, that the atmospheric turbulence is characterized by a power spectrum with a $-11 / 3$ power law coefficient (see Eq. 28). While the negative sign ensures that less power is contained in the high-spatial frequencies, there is no limit to how fine the turbulence structures get in this description: to correct for everything would require a deformable mirror with an infinite number of active elements, which is not a realistic solution. In practice, a DM 


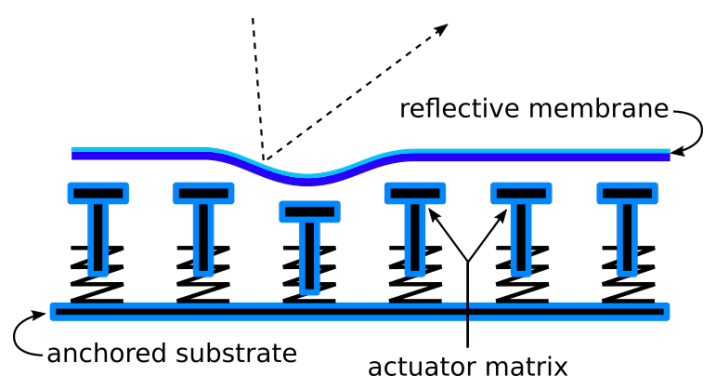

Figure 23. Schematic representation of the layout of a row of actuators pushing or pulling on a continuous reflective membrane. The combined effect of the motion of all actuators gives the ability to generate complex shapes to the deformable surface to compensate the effect of upstream aberrations.
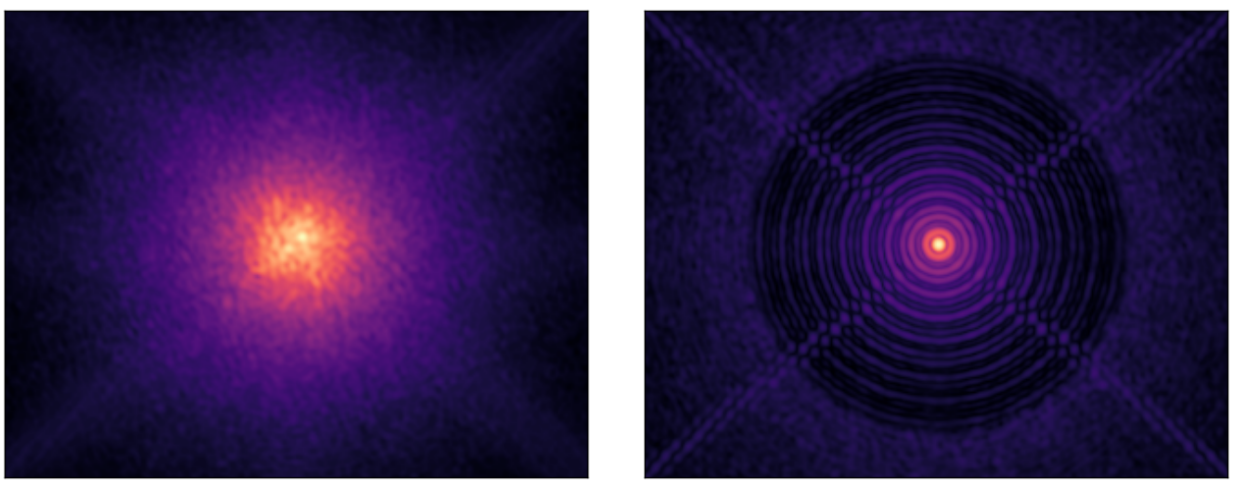

Figure 24. Long exposure acquired through the atmosphere. Left: long exposure acquired in the absence of adaptive optics. Unlike the sharp speckle pattern presented in Fig. 21, the features of this image are washed out by the many turbulence realisations forming a wide halo. Right: long exposure at the focus of an XAO system. The circular region surrounding the now clearly defined and well corrected PSF core at the center of the image marks the domain of spatial frequencies that the DM is able to compensate.

is made of a finite number of actuators used either to deform a thin reflective membrane or to push and orient non-deformable mirror segments. For the DMs we are concerned with here, the actuators are laid out on a regular grid. Figure 23 proposes a schematic representation for the implementation of a row of actuators. The total number of actuators distributed across the pupil of the instrument will determine the finesse of the correction one can expect to produce: the DM will act like a filter that can attenuate the atmospheric phase screen up until a cut-off spatial frequency $f_{c}$ imposed by the number of actuators across aperture. For the high-contrast imaging application, the wavefront quality requirement drives the need for a large number of actuators, of the order of a few thousand for an 8-meter aperture. With such a large number of actuators, one sometimes talk about extreme adaptive optics or XAO. The effect of this cut-off spatial frequency is visible in the right panel of Figure 24: a clean circular area surrounds the well corrected PSF core around which one can distinguish the diffraction rings. The DM used to produce this simulated image features $N=50$ actuators across the aperture, pushing the correction radius to $r_{c}=N / 2=25 \lambda / D$. For an 8 -meter 
aperture observing in the H-band, this translates into a control region that is $\sim 1$ arc second. Beyond this correction radius, the power contained in the high-spatial frequency content of the PSF is no-longer compensated and contributes to the formation of another halo.

\section{Extreme adaptive optics}

The high quality wavefront correction required for high-contrast imaging pushes for AO systems with a large number of actuators, tightly integrated with the coronagraph: the integration of the high contrast imaging constraint to the wavefront control loop marks the specificity of what is now known as extreme adaptive optics. When deforming the mirror, the distribution of complex amplitude $A$ across the aperture of the instrument is given by:

$$
A(x, y)=P(x, y) \times e^{i \Phi(x, y)}
$$

where $P(x, y)$ describes the shape of the telescope aperture and $\Phi(x, y)$ the distribution of phase from the combined effect of the atmosphere and the correction by the DM. In the XAO regime, the amplitude of the residual phase is small enough to justify linearizing the complex amplitude:

$$
A(x, y) \approx P(x, y) \times(1+i \Phi(x, y)) .
$$

Perfect control of the aberrations would mean $\Phi(x, y)=0$ over the entire aperture, leaving only the unity factor resulting in the static diffraction pattern. This linearized form makes it possible to separate the static and the dynamic components of the diffraction, respectively corresponding to the real and imaginary parts of Eq. 30. This form can in turn be used to compute an approximation for the PSF in the low-aberration regime:

$$
\operatorname{PSF}(\alpha, \beta)=\|\mathcal{F}(P(x, y))\|^{2}+\|\mathcal{F}(P(x, y) \times \Phi(x, y))\|^{2} .
$$

Figure 20 showed how the Lyot-coronagraph manages to attenuate the static diffraction of an on-axis source, but nevertheless leaves a residual: this coronagraph is not perfect. However, other more recent coronagraphic solutions like the vortex [27] and the PIAA [3] coronagraphs are closer to being able to completely get rid of this diffraction term [28]. With such designs, the post-coronagraphic residuals for the on-axis source are dominated by the wavefront errors. Coronagraph designs can therefore be benchmarked against the so-called perfect coronagraph, a theoretical design producing the following on-axis coronagraphic image:

$$
\mathrm{I}(\alpha, \beta)=\|\mathcal{F}(P(x, y) \times \Phi(x, y))\|^{2},
$$

for which the static diffraction term originally present (see Eq. 31) has deliberately been removed. In the absence of aberrations, the perfect coronagraph provides a perfect extinction of the on-axis source. In the presence of aberrations, the perfect coronagraph leaks and some residual starlight finds its way to the final focal plane. Figure 25 shows one example of application of this perfect coronagraph formula, for an AO-corrected wavefront residual of 50 $\mathrm{nm}$. The details of this computation will depend on the statistics of the residual aberrations. In Eq. 32, we see the phase $\Phi$ squared appearing as a scaling factor for the wavefront aberration residual light in the post-coronagraphic focal plane. A close look at the well-corrected area of the coronagraphic image shown in Fig. 25 shows that the coronagraphic leak does look like a scaled-down copy of the original PSF. For a given RMS level of residuals $\alpha$ (expressed in nanometers) one can therefore predict a focal contrast improvement factor $c_{b}$ (for contrast boost) at any place in the focal plane: 

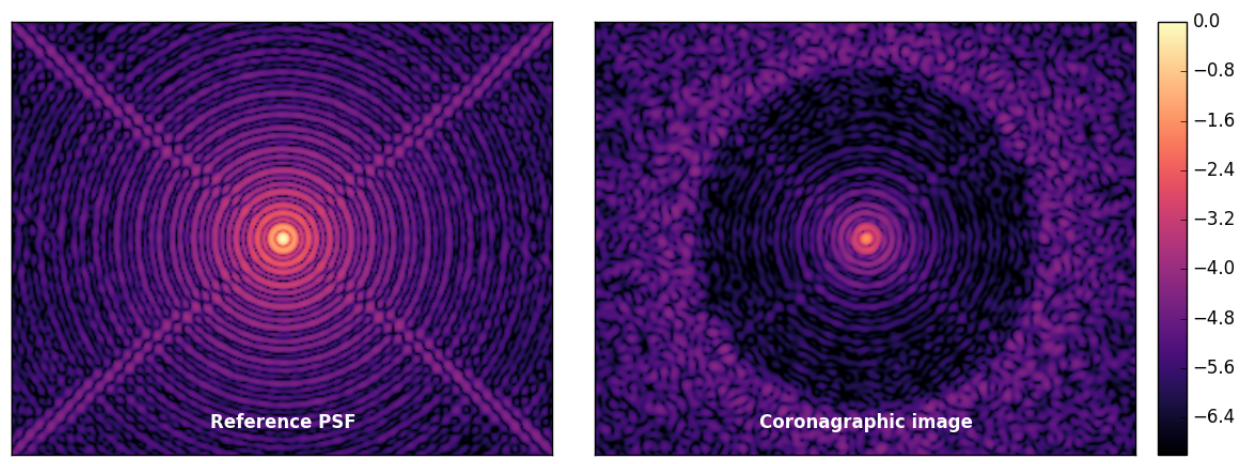

Figure 25. Example of image computation using the perfect coronagraph formula. Left panel: a noncoronagraphic PSF, affected by $50 \mathrm{~nm}$ RMS residual wavefront errors. Right panel: a coronagraphic image computed for the same instrument pupil aberration. Both images share the same logarithmic stretch and colorbar.

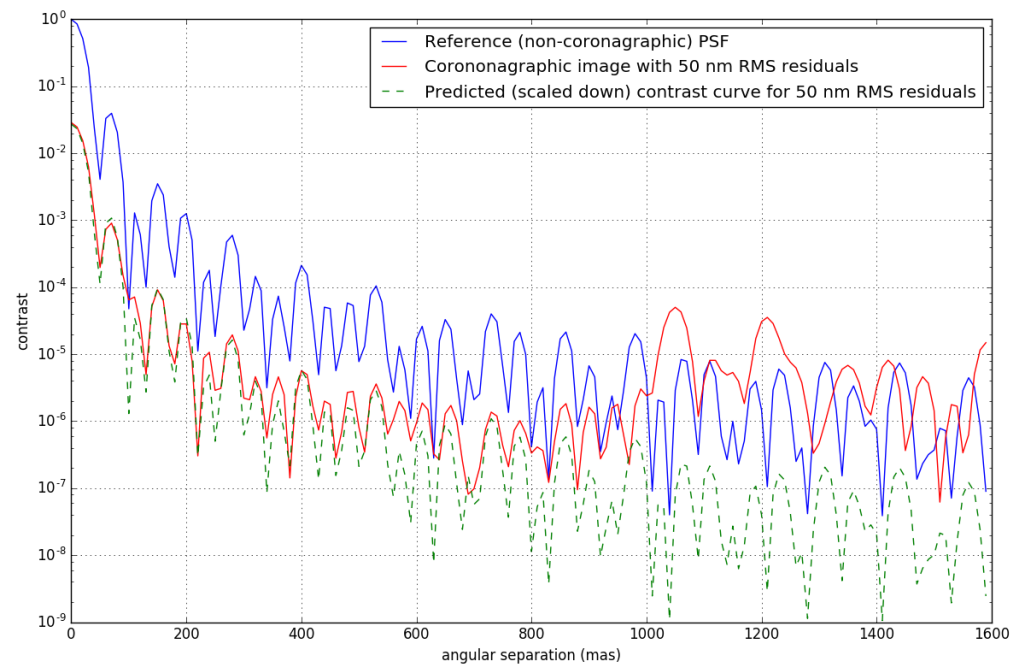

Figure 26. Contrast curves for the perfect coronagraph, in the presence of $50 \mathrm{~nm}$ RMS residual wavefront aberration. Three curves are represented, corresponding to radial profiles of the images provided in Fig. 25: the reference (non-coronagraphic) PSF in blue and the corresponding coronagraphic equivalent in red. The green dashed line is a copy of the blue curve, scaled down by the factor $c_{b}$. Over the first $\sim 500$ mas of the plot, the match between the red and the dashed green curves is quite good.

$$
c_{b}=\frac{1}{\sqrt{2}}\left(\frac{2 \pi \alpha}{\lambda}\right)^{2} .
$$

For the simulated $50 \mathrm{~nm}$ RMS residual wavefront error shown in Fig. 25, one therefore expects a $c_{b} \approx 0.027$ contrast improvement over the original non-coronagraphic PSF. On Fig. 26 , one can verify that over the first $\sim 500$ mas of the corrected area, this approximation does match reasonably well the simulated image. 
This model can be further refined [29] and used to derive the statistics of the wave amplitude at each point in the focal plane [30], and evaluate the relevance of high-contrast devices in general. What this kind of study shows is that it is not useful to design a coronagraph that attenuates the on-axis PSF of a bright star further than the amount of residual speckles expected for a given amount of residual wavefront aberrations. It is the performance of the AO that will drive how far a coronagraph can help you go, and we will now look into ways the AO performance can unfortunately throw you off target.

\section{Calibration of biases}

So far, the description has been assuming that the AO system was doing the right thing: to flatten the wavefront so as to help the coronagraph effectively erase the on-axis static component of the diffraction of a bright star. This turns out to be a somewhat naive assumption, due to a simple but fundamental limitation. The wavefront sensor (refer back to Fig. 22) can indeed only sense the aberrations introduced by the instrument optics all the way down to the optics that splits the light between the sensor and the downstream instrument. The $\mathrm{AO}$ is therefore understandably oblivious to anything affecting the light on the instrument path after this split which results in practice in a non-common path error. Much care is obviously taken while initially setting up instruments, to minimize any non-common path error however, ground based instruments are not immune to minute temperature changes and mechanical flexures. Given the very strong dependence of the coronagraphic rejection on input wavefront quality, the least amount of non-common path error will considerably reduce the discovery potential of any high-contrast imaging instrument.

These techniques are victims of their own success: before the generalized use of $\mathrm{AO}$, the overall quality of astronomical images produced by a well built instrument was dominated by random atmospheric induced errors. The progressive deployment of $\mathrm{AO}$ and the improvement of its performance has reduced the contribution of random errors, resulting in an improved precision. But when the amplitude of random errors is reduced, the effect of small but systematic biases affecting our accuracy become more apparent (see Fig. 27). XAO-fed high contrast imaging and long baseline interferometry make it possible to enter the realm of very high-precision observations: the search and compensation of instrumental biases is becoming more important than ever.

Despite the very high quality wavefront control (50 nm RMS only) used to produce the simulated coronagraphic image in Fig. 25, the control region features a large number of speckles amongst which a faint genuine companion to the bright star could hide. If induced by random AO residual errors, the amplitude noise induced by these structures can be reduced simply by accumulating enough data. However if some static or quasi-static speckle structures induced by an aberration that is not seen by the wavefront sensor persist over long time-scales, the detection of faint planetary companions is compromised. We are going back to the important question highlighted in the introduction of this paper: any speckle-like feature present in the image can either be a diffraction induced artefact or a genuine structure of the target being observed.

The non-common path error turns out to be one of the dominant limitations of highcontrast imaging instruments as faint systematic structures are reported to survive in images over timescales stretching as far as $\sim 1$ hour. The simplest calibration procedure employed in astronomy consists in using images acquired on a reference object of known characteristics (ideally a featureless single star), observed under conditions as identical as possible to those used for the object of interest, and to subtract the calibration image from the image of the target of interest. Using the shooting analogy used in Fig. 27, we would use a series of shots aiming at the center of the target to figure out how off our aim really is, before going for the 


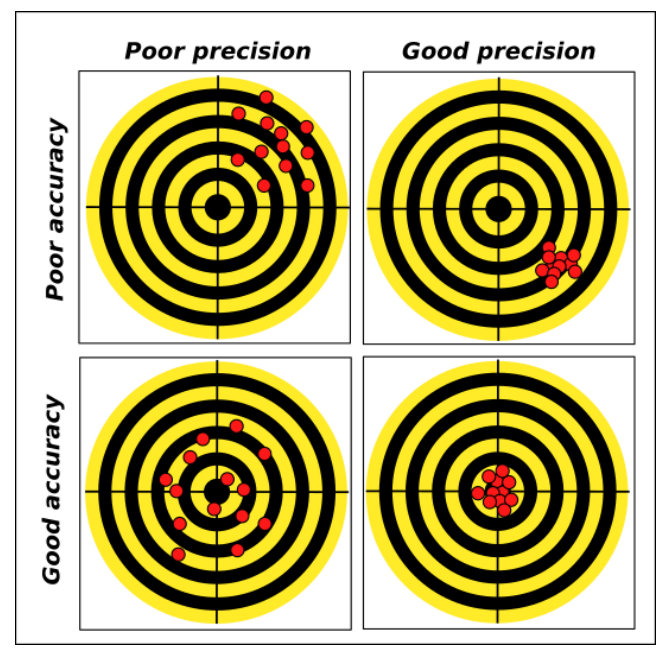

Figure 27. Precision and accuracy are two important statistical concepts that characterize all measurements. As we improve the quality of our instrumentation, usually reducing the precision of the measurements they produce, the impact of systematic bias affecting the accuracy of our conclusions becomes an essential element to take into consideration. Much energy should be spent on understanding the origin of biases and figuring out how to calibrate them out.

big game. This strategy is common for the interpretation of AO-corrected images as well as for optical interferometry for which it is often advised to observe more calibrators than targets of interest.

While reasonably effective the calibrating potential of this method remains limited: it is difficult to guarantee that observing conditions are indeed strictly identical when going from a science target to a calibrator. The repointing of the telescope, the small differences in elevation, spectral type and evolutions of the atmospheric seeing will eventually translate into biases of their own. For high-contrast imaging, other approaches are available that do not require to alternate observations on a target interest with those of one or more calibration stars: it is indeed possible to take advantage of the field rotation experienced when an instrument is installed at the focus of an alt-azimuthal telescope. When following a target as it transits across the local meridian with one such instrument, the target appears to rotate while the residual diffraction induced by the instrument corrected by the AO but still affected by the non-common path error, remains stable. The relative rotation between the observed scene and the residual diffraction pattern can be used to distinguish spurious diffraction features from genuine structures in a series of images. This approach is referred-to as angular differential imaging or ADI [33] and has led to a wide variety of algorithms such as LOCI [34]. Any type of observation that include some form of diversity such simultaneous imaging in two spectral bands (spectral differential imaging or SDI) or in two polarization states (polarized differential imaging or PDI), theoretically makes it possible to calibrate out systematic effects that bias observations. One must however remain attentive to the implementation details, as these techniques end up relying on further splits of the light path, which can become a source of non-common path. To account for all possible biases during such observations requires a multiple tier calibration procedure, that includes the ability to swap light paths, an example of which is given in [35]. 

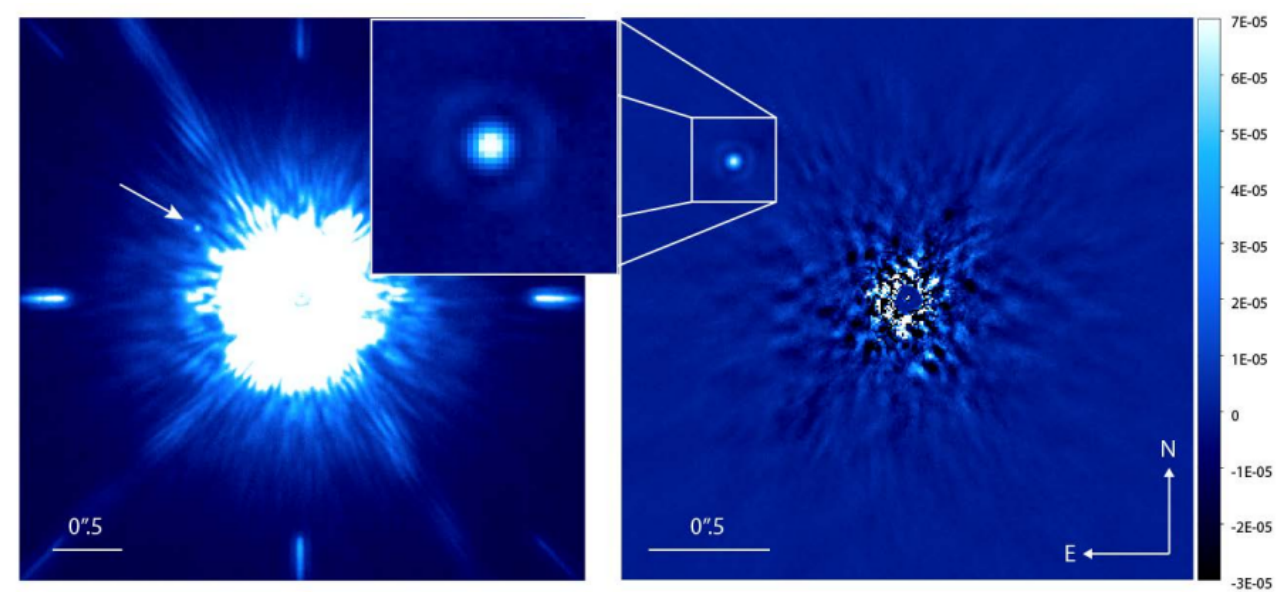

Figure 28. Illustration of the impact of post-processing (from [31]) on high-contrast observations with a vortex-coronagraph by the SCExAO instrument [32]. The left hand side panel features shows a raw 15 second exposure of $\kappa$-And and the right hand side panel shows the impact of the ADI quasi-static speckle pattern subtraction. The highlighted companion, that could be mistaken for a speckle visible in the raw image, is clearly above the noise level after the post processing has been applied.

More recent approaches give a new spin to the idea of using calibrators, by relying on the principal component analysis of a library of reference PSFs [36], which provides performance comparable to ADI-inspired approaches. Finally, it is also possible to take the information contained in AO-corrected images (albeit not coronagraphic ones), and to project it onto a sub-space (called the Kernel) that filters out aberrations [37]. The approach is reminiscent of the closure-phase technique used in interferometry $[38,39]$, but is now applicable to AOcorrected images [40], and is particularly relevant for detection near the diffraction limit (around $\sim 1-2 \lambda / D$ ). Regardless of the algorithmic details at work, Figure 28 shows the impact the post-processing has on high-contrast imaging by comparing a single raw coronagraphic image to the result of post-processing of a 10-minute series of images: the impact of the speckle subtraction is spectacular and sometimes contribute as much if not more than the coronagraph itself.

\section{Focal-plane based wavefront control?}

Unless high-contrast imaging solutions that are intrinsically robust to weak amounts of aberrations do emerge, better calibration stategies must be employed if a performance improvement is desired. The importance for a good calibration of systematic effects in coronagraphic images will grow as the quality of the upstream AO correction keeps on improving. One needs to find, at the level of the focal plane, a discrimination criterion that will make it possible to distinguish a genuine struture in the focal plane from a spurious diffraction induced speckle. The introduction to this paper already hinted at one possibility, relying on the ability to measure the degree of coherence of the structure in question. Section 2 introduced the idea of coherence as the ability of light to interfere. Given the two important coherence properties of astronomical sources: the fact that the light of an unresolved point source is perfectly coherent while the light of distinct point sources is incoherent, can be used to discriminate speckles from planets in an image. 

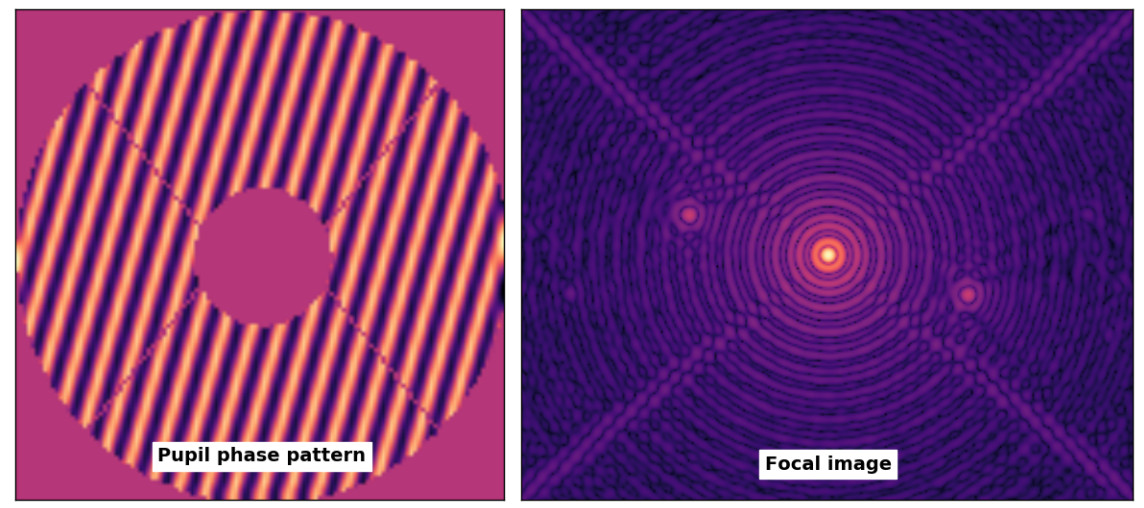

Figure 29. Left: sinusoidal modulation of the wavefront across the instrument pupil by the high-order deformable mirror. Right: the resulting point spread function. The original on-axis diffraction pattern is flanked by two high-contrast replicas at a distance of $\sim 18 \lambda / D$, given by the number of cycles across the aperture.

Deliberate modulation of the starlight synchronized with acquisitions by the focal plane camera form the basis for an ideal coherence test that will discriminate the true nature of the high-contrast features present in an image. The deformable mirror can indeed be used to send additional light atop of whatever is currently in the focal plane and the camera can be used to diagnose the degree of coherence of the light recorded in the live image.

The grid structure of the DM actuators (see Figure 23) used in XAO systems makes them particularly suited to the generation of sinusoidal modulation patterns. When one such modulation is applied, the DM behaves like a diffraction pattern and displaces some of the starlight that would otherwise be transmitted on-axis (and possibly attenuated by the coronagraph) at a distance that is proportional to the number of cycles across the aperture.

With $N$ actuators across one aperture diameter, the highest spatial frequency one can reach corresponds to a state where every other actuator is pushed up with the others pushed down: the sinusoidal wave thus generated contains $n_{c}=N / 2$ cycles across the aperture. This is what sets the cut-off spatial frequency of AO introduced in Section 6. A deformation $\Delta$ of respectively $k_{x}$ and $k_{y}$ cycles (both $\leq n_{c}$ ) along the $x$ and $y$ directions of the image is equal to:

$$
\Delta(x, y)=a \times \sin \left(2 \pi\left(k_{x} x+k_{y} y\right) / D+\phi\right)
$$

where $a$ is the amplitude of the modulation (typically expressed in microns or nanometers) and $\phi$ the phase of that modulation. For a small modulation amplitude $\alpha$, the complex amplitude $A(x, y)$ induced by this deformation can be linearized (like for Eq. 30) $)^{5}$ :

$$
\begin{aligned}
A(x, y) & =P(x, y) \times \exp (i 4 \pi / \lambda \times \Delta(x, y)) \\
& \approx P(x, y) \times\left(1+i 4 \pi a / \lambda \times \sin \left(2 \pi\left(k_{x} x+k_{y} y\right) / D+\phi\right)\right)
\end{aligned}
$$

\footnotetext{
${ }^{5}$ The global scaling factor is here $4 \pi / \lambda$ and not $2 \pi / \lambda$ as one might have expected. This $\times 2$ factor is there to take into account the fact that we are dealing with a reflection off a mirror: a $\Delta$ mechanical deformation of the surface induces a $2 \Delta$ deformation of the wavefront.
} 
We know that a Fourier transform relates the distribution of complex amplitude in the pupil to that in the focal plane, and can relate values of $a$ and $\phi$ to the properties of speckles in the focal plane. If one knows the Fourier transform of the sine function:

$$
\mathcal{F}(\sin (2 \pi k x))=\frac{1}{2 i}(\delta(u-k)-\delta(u+k)),
$$

where $\delta(u)$ is the Dirac distribution, then the Fourier transform of Eq. 36 will write as:

$$
\hat{A}(\alpha, \beta)=\hat{P}(\alpha, \beta) \otimes\left(\delta(\alpha, \beta)+\frac{2 \pi a}{\lambda}\left(e^{i \Phi} \delta\left(\alpha-k_{x}, \beta-k_{y}\right)-e^{-i \Phi} \delta\left(\alpha+k_{x}, \beta+k_{y}\right)\right)\right),
$$

using angular coordinates $\alpha$ and $\beta$ expressed in units of $\lambda / D$. A detector located in the focal plane will record the square modulus of this expression. Each $\delta$ function (convolved by the Fourier transform of the aperture $\hat{P}$ ) marks the location of one PSF. Eq. 38 therefore allows you to predict that the focal plane will feature two replicas of the original on-axis PSF, at positions given by the number of cycles $k_{x}$ and $k_{y}$. The two replicas are characterized by reference complex amplitudes: $v_{1}=(2 \pi a / \lambda) \times e^{i \Phi}$ and $v_{2}=(2 \pi a / \lambda) \times e^{i(\pi-\Phi)}$. An example of image showing this is shown in Fig. 29 for $\sim 18$ cycles, resulting in a pair of symmetrical replicas of the on-axis PSF at $\sim 18 \lambda / D$. Whereas the number of cycles imposes the location of the replicas, the amplitude of the modulation $a$ sets the contrast relative to the original PSF, which is given by:

$$
c=(2 \pi a / \lambda)^{2} .
$$

Plugging in a sinusoidal phase modulation of amplitude $a=50 \mathrm{~nm}$ therefore produces in the $\mathrm{H}$-band $(\lambda \approx 1.6 \mu \mathrm{m})$ a pair of replicas of contrast $c \approx 4 \times 10^{-2}$ which may seem surprisingly bright to the reader. Figure 26 indeed presented for the perfect coronagraph in the presence of a similar level of RMS error, considerably more favorable contrasts. The difference between the two scenarios lies in the structure of the residual phase noise: near random in the case presented in Fig. 26 which distributes the total amount of light associated to the RMS over the control region, or highly structured in the sinusoidal modulation scenario, that focuses the diffracted light onto two specific locations. In addition to the residual RMS given by an $\mathrm{AO}$ or XAO system, it is therefore also important to understand how the residuals are distributed across the aperture.

So far, we've accounted for the number of cycles $k_{x, y}$ and the modulation amplitude $a$ but not for the $\Phi$ and $\pi-\phi$ phase of the replicas remains: if one were to ignore the convolution operation by $\hat{P}$, taking the square modulus of second term of Eq. 38 would make those phase terms disappear as the $e^{i \Phi}$ factor disappears, suggesting that the phase of the replicas does not matter. The convolution will however bring diffracted light over the area covered by the replica. We have starlight landing atop of starlight: the two contributions will interfere with one another. If the light of an incoherent source is present (ie. a planet or one local disk structure), then the added light will not interfere with this structure: the two intensitise will simply add. Depending on the phase difference between the replica and the speckles or diffraction features already present in the focal plane, the interference can either be constructive or destructive, which leads to an interesting prospect: the possibility of improving the raw contrast of images by tweaking the shape of the deformable mirror. Earlier, it was pointed out that the job of AO feeding a classical imaging system is to flatten the wavefront so as to improve the overall image quality: for a high-contrast imager, the optimal strategy is no longer to flatten the wavefront but to improve the contrast in the focal plane, which can drive the DM to shape the DM quite far from flat. This idea was first envisioned for space [41] and has led to a 
series of sophisticated algorithms such as speckle-nulling [42], electric field conjugation [43] or stroke minimization [44]. If the wavefront sensing systems used in modern AO systems tolerate the idea of being driven away from a flat reference wavefront, then this approach becomes implementable on ground based high-contrast imaging instruments.

To produce a fully destructive interference that would result in a local reduction of the local intensity in the image, the complex amplitude of the added replica must match that of the already present structure, with the same amplitude but with opposite phase.

In practice, one does not direcly measure the contrast of speckles: one will primarily access to a local intensity $I_{0}$ which we know will be proportional to the square modulus of the speckle complex amplitude:

$$
I_{0}=\gamma \times\left\|a_{0} e^{i \Phi_{0}}\right\|^{2}=\gamma \times\left\|a_{0}\right\|^{2},
$$

where the proportionality constant $\gamma$ will depend on the brightness of the target and the exposure time and will therefore have to be regularly estimated and controlled. While the intensity associated to speckle gives us a proxy for its amplitude $a_{0}$, its phase $\Phi_{0}$ remains unknown. To determine it, one can use a probing approach, which consists in following the evolution of the local intensity as the speckle interferes with a probe speckle of known, stable amplitude $a$ and variable phase $\Phi$. The intensity of the coherent sum of these two complex amplitudes is the result of a classical two-wave interference equation:

$$
\begin{aligned}
I(\Phi) & =\gamma \times\left\|a_{0} e^{i \Phi_{0}}+a e^{i \Phi}\right\|^{2} \\
& =\gamma \times\left(a_{0}^{2}+a^{2}+2 a_{0} a \cos \left(\Phi_{0}-\Phi\right)\right) .
\end{aligned}
$$

This interference function is evaluated for an adaptable number of probes with a phase $\Phi$ uniformly sampled between 0 and $2 \pi$ radians and at least three distinct values of $\Phi$ are required to constrain the values of $a_{0}$ and $\Phi_{0}$. In practice, a finer sampling minimizes the sensitivity to high temporal frequency phase noise (overall jitter and AO dynamic residuals). An example of modulation is represented in Figure 30. It compares the original intensity level marked by the horizontal red line to the blue curve recording the evolution of the phase modulation. When the probe is in phase with the original speckle $\left(\Phi=\Phi_{0}\right)$, the local intensity is quadrupled. When the probe is in phase opposition with the original speckle $\left(\Phi=\Phi_{0}+\pi\right)$, the local intensity can be brought to zero.

With four probes, with phases $0, \pi / 2, \pi$ and $3 \pi / 2$, an analytical solution exists to directly measure the complex amplitude of the speckle: this is the so-called ABCD-method. A more general solution is however possible, that is compatible with an arbitrary number $N$ of phases (with $N \geq 3$ ). It boils down to a parametric model fit of the modulation curve. In addition to $\mathbf{I}_{S}$ the vector of $N$ intensities recorded during the probing sequence, one can precompute a separate vector $\mathbf{W}$ that contains the consecutive powers of the $N^{\text {th }}$ root of unity $w_{N}=e^{i 2 \pi / N}$. The value of the phase $\Phi_{0}$ is directly given by the argument of the dot product between these two vectors:

$$
\Phi_{0}=\arg \left(\mathbf{I}_{S}^{\top} \cdot \mathbf{W}\right)
$$

while the visibility modulus $\Gamma(0<\Gamma<1)$ characterizing the modulation described by Eq. 30 is related to the modulus of the dot product:

$$
\Gamma=\frac{2 a a_{0}}{a^{2}+a_{0}^{2}}=\frac{2}{N}|| I_{S}^{\top} \cdot W||
$$




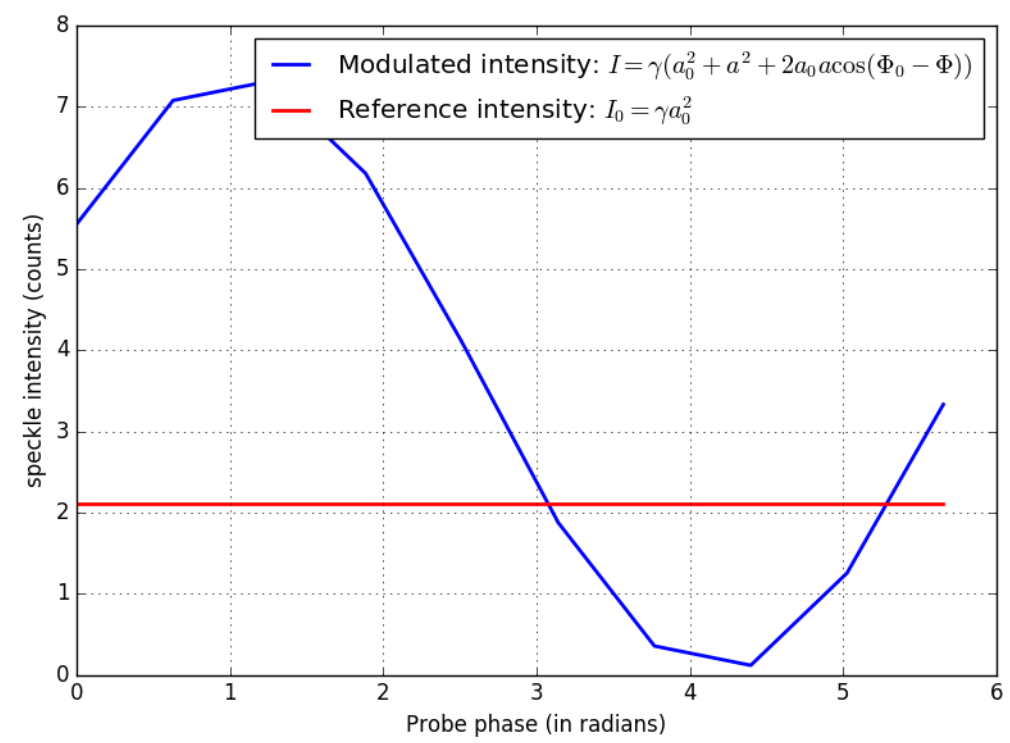

Figure 30. Modulation of the speckle intensity (expressed in units of detector counts) as a function of speckle probe phase. The red curve marks the intensity of the original speckle for which the phase is unknown. The blue curve is the result of the addition of a probe speckle of variable phase $\Phi$. The observed modulation confirms that the speckle is a coherent (ie. contains starlight) structure: one phase of the added speckle ( $\Phi \sim 4.5$ radians) attenuates the local intensity, resulting in an improved local raw contrast.

The amplitude $a_{0}$ of the original speckle is one of the two roots of the following quadratic equation:

$$
a_{0}^{2}-\frac{2 a}{\Gamma} a_{0}+a^{2}=0
$$

which are given by:

$$
a_{0}=\frac{a}{\Gamma}\left(1 \pm \sqrt{1-\Gamma^{2}}\right) .
$$

The amplitude $a$ of the probe is selected to be as close as possible to the amplitude of the speckle $a_{0}$, so as to maximize the visibility modulus $\Gamma$, which results in an improved sensitivity to the properties of the speckle. Because we can't afford to make a mistake that will amplify the speckle present if we pick the wrong amplitude, one solution is to systematically buff up the probe (for instance by $5 \%$ ): some sensitivity is lost but we can be sure that the solution (from Eq. 46) with the minus sign will always be the right one.

Using this algorithm, it is possible to create a closed-loop focal-plane based wavefront control loop that modifies the reference position of the DM to create a higher contrast area within the control region. Note that while the description of this technique looked at a single speckle, the algorithm can be multiplexed and simultaneously probe dozens or even hundreds of speckles (the exact number depends on the number of actuators available), making it much more efficient. Note that instead of a temporal modulation of the coronagraphic speckle field inside the control region, spatial modulation is possible: the self-coherent camera (SCC) [45] relies on this idea. Instead of acquiring a sequence of images before applying a correction, 


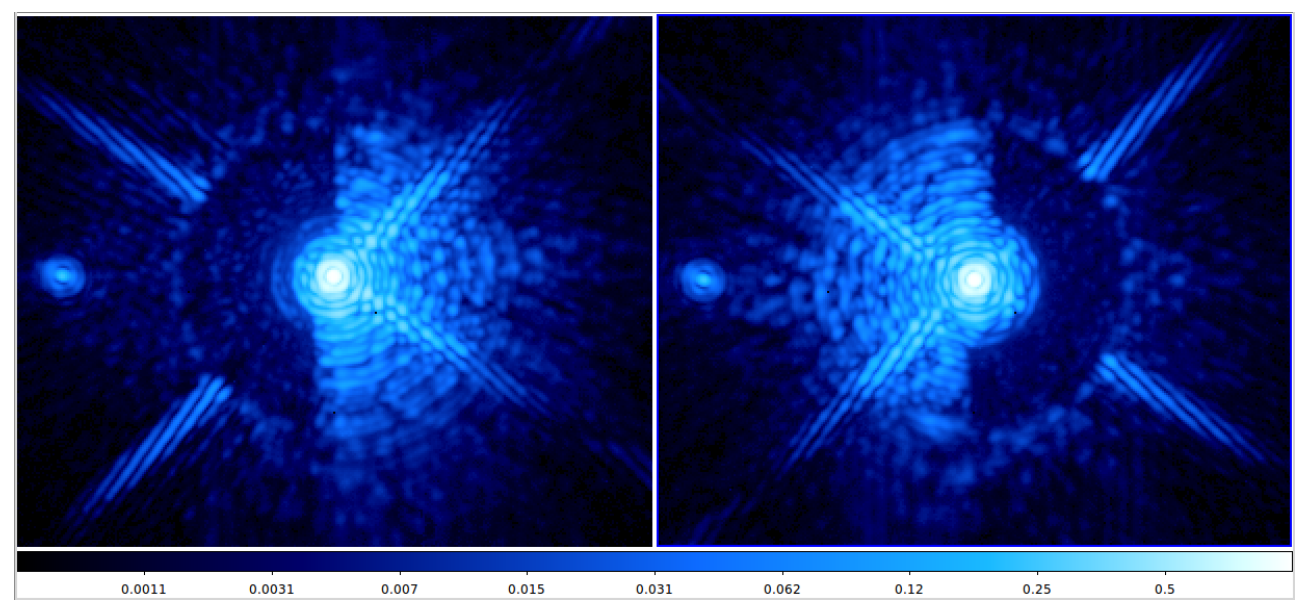

Figure 31. Result of two speckle nulling experiments carried out on the SCExAO instrument on an internal calibration source without a coronagraph. For each image, one can see a high-contrast region was created by the speckle nulling loop on one side of the field of view only. Although it is less obvious on these images, on the opposite side, the speckles and diffraction features of the PSF are amplified as a result of the speckle nulling.

the focal plane must be oversampled while a reference beam of starlight is uniformly projected over the control region: the speckles feature fringes that directly encode the complex amplitude properties of the speckles.

Speckles in the focal plane have two origins: they can either be induced by pupil phase aberrations, or can be induced by the geometry of the aperture itself if the coronagraph is absent or if it is imperfect: one can always think of diffraction rings and spikes as being made up of a coherent sum of individual speckles: we can refer to these as induced by pupil amplitude aberrations. It sounds fair game to try and compensate for phase aberration induced speckles by phase modulations, but what of pupil amplitude induced ones? By bending the wavefront, the DM can only redistribute the energy in the focal plane and not make it disappear: when it corrects phase induced speckles, the energy associated to these speckles gets injected back into the original PSF; when it corrects an amplitude induced speckle on one side of the field, it amplifies the speckle on the opposite side. Figure 31 shows the result of two speckle nulling experiments done on the SCExAO instrument in a non-coronagraphic mode. The high-contrast region created by the successive attenuation of speckles covers half of the control region. The appropriate DM modulation produces a high-contrast region in the focal plane, an effect that is similar to what apodization achieves (see Sec. 5.1). The use of static apodizing phase plates achieving a similar effect [46] has been successfully exploited on-sky. These phase plates benefit from advantages that render them fairly achromatic [47]. But like all static high-contrast imaging devices, they are not immune to biases and a focal plane feedback loop remains essential.

If the same deformable mirror is simultaneously driven by the upstream $\mathrm{AO}$ that tends to flatten the wavefront and this focal plane based control that deliberately pushes it away from the flat, to improve the raw contrast in the image, conflicts may occur. One (easy but expensive!) solution may be, in future implementations, to rely on two distinct mirrors for the $\mathrm{AO}$ and the high-contrast. The other (cheaper but more difficult) requires the upstream AO system to agree with the idea of stabilizing the wavefront away from flat. This is the object 

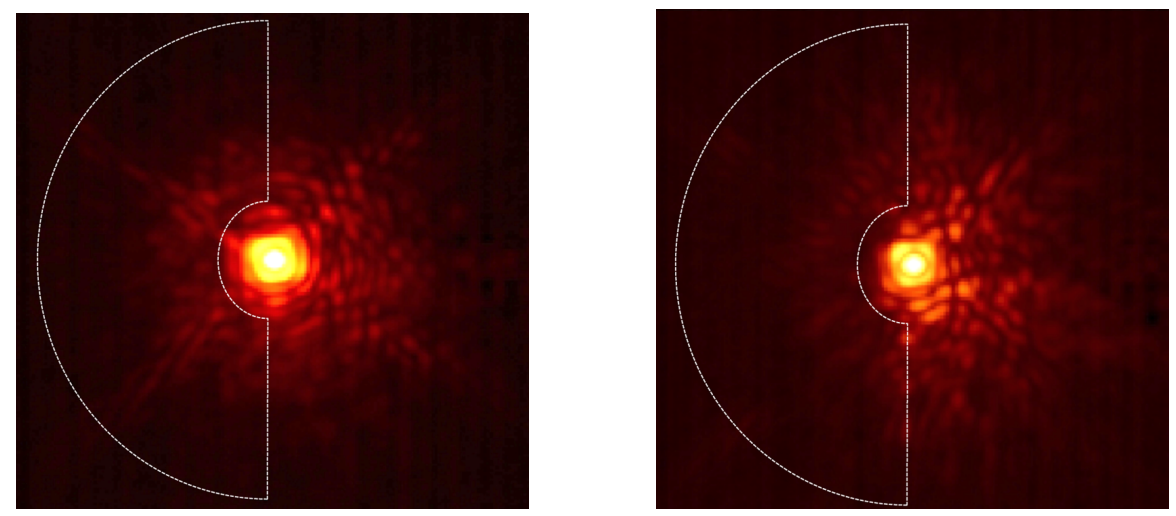

Figure 32. Example of on-sky speckle nulling experiment with the SCExAO instrument without a coronagraph (from [32]). Left: the starting point of the algorithm, after the initial lock of the upstream AO system. Right: after a few minutes of speckle nulling closed loop operation, the left hand side of the field is effectively darker. The D-shaped control region over which the loop is operating is highlighted.

of ongoing work: an exemple of partial speckle nulling correction obtained on sky also with the SCExAO instrument is presented in Figure 32.

\section{Conclusion}

One of the goals of this lecture was to highlight the formalism and properties that optical interferometry and high-contrast imaging have in common: we first focused on the notion of coherence, most often invoked in the sole context of interferometry, since this technique directly aims at measuring it. The fundamental coherence properties of astronomical sources however also make it possible to explain how images acquired by telescopes form. They also explain what information can be extracted from images dominated by diffraction features. The link between the two techniques runs strong: the Van Cittert Zernike theorem, used at the very heart of interferometry to relate the measurements of complex visibilities to the properties of astrophysical sources (refer to the lecture by Prof. Jean Surdej in this book) can be understood as a Fourier-centric equivalent of the image - object convolution relation.

Equipped with this formal background, we took a closer look at high-contrast imaging and the principles behind the optical techniques of pupil apodization and coronagraphy that attempt to beat down the photon noise of the bright star and improve the detectability of high-contrast sources in their vicinity. We now know that these solutions can only reduce the photon noise associated to the static diffraction figure of the instrumental chain. In the presence of residual aberrations, their performance rapidly degrades and their benefit becomes marginal. State of the art extreme adaptive optics systems, manage to bring the wavefront residual errors down to a few tens of nanometers, but systematic biases, mostly associated to non-common path errors do survive over long time-scales and limit the discovery potential of high-contrast imaging instruments. Sophisticated post-processing techniques do manage to calibrate some of these systematics and have considerably contributed to the direct imaging of a few planetary systems featuring bright planets. There are still orders of magnitude to overcome to directly image the large number of mature planets theoretically within the grasp of ground based telescopes. Before having to resort to post-processing, closed-loop feedback from the focal plane while carrying out the observations seems like a reasonable 
way to compensate for biases, and design systems that better answer the question: speckle or planet? Going full circle back to the notion of coherence, the example of iterative speckle nulling was described in higher details. Other techniques and algorithms are also possible and may prove more efficient to implement as they mature and adapt to the tough telescope environment. The robustness of the speckle nulling approach however makes it an attractive next step in the elimination of biases for high-contrast imaging.

\section{References}

[1] N.J. Kasdin, R.J. Vanderbei, D.N. Spergel, M.G. Littman, ApJ, 582, 1147 (2003)

[2] R. Soummer, C. Aime, P.E. Falloon, A\&A, 397, 1161 (2003)

[3] O. Guyon, A\&A, 404, 379 (2003), arXiv: astro-ph/0301190

[4] O. Guyon, E.A. Pluzhnik, R. Galicher, F. Martinache, S.T. Ridgway, R.A. Woodruff, ApJ, 622, 744 (2005), arXiv: astro-ph/0412179

[5] A. Carlotti, R. Vanderbei, N.J. Kasdin, Optics Express 19, 26796 (2011)

[6] G. Guerri, J.B. Daban, S. Robbe-Dubois, R. Douet, L. Abe, J. Baudrand, M. Carbillet, A. Boccaletti, P. Bendjoya, C. Gouvret et al., Experimental Astronomy 30, 59 (2011)

[7] B. Lyot, ZAp, 5, 73 (1932)

[8] F. Roddier, C. Roddier, PASP, 109, 815 (1997)

[9] R. Soummer, K. Dohlen, C. Aime, A\&A, 403, 369 (2003)

[10] D. Mawet, P. Riaud, O. Absil, J. Surdej, ApJ, 633, 1191 (2005)

[11] D. Rouan, P. Riaud, A. Boccaletti, Y. Clénet, A. Labeyrie, PASP, 112, 1479 (2000)

[12] N. Murakami, R. Uemura, N. Baba, J. Nishikawa, M. Tamura, N. Hashimoto, L. Abe, PASP, 120, 1112 (2008)

[13] R. Soummer, ApJL 618, L161 (2005), astro-ph/0412221

[14] A. Kolmogorov, Akademiia Nauk SSSR Doklady 30, 301 (1941)

[15] D.L. Fried, Journal of the Optical Society of America (1917-1983) 56, 1372 (1966)

[16] V.I. Tatarskii, Wave Propagation in Turbulent Medium (McGraw-Hill, 1961)

[17] V.I. Tatarskii, The effects of the turbulent atmosphere on wave propagation (1971)

[18] A. Labeyrie, A\&A, 6, 85 (1970)

[19] C. Aime, European Journal of Physics 22, 169 (2001)

[20] H.W. Babcock, PASP, 65, 229 (1953)

[21] G. Rousset, J.L. Beuzit, The COME-ON/ADONIS systems (1999)

[22] F. Roddier, Appl. Opt.27, 1223 (1988)

[23] R. Ragazzoni, Journal of Modern Optics 43, 289 (1996)

[24] O. Guyon, ApJ, 629, 592 (2005), arXiv: astro-ph/0505086

[25] F. Roddier, Adaptive Optics in Astronomy (2004)

[26] J.W. Hardy, Adaptive Optics for Astronomical Telescopes (1998)

[27] D. Mawet, E. Serabyn, K. Liewer, R. Burruss, J. Hickey, D. Shemo, ApJ, 709, 53 (2010), 0912.2287

[28] O. Guyon, E.A. Pluzhnik, M.J. Kuchner, B. Collins, S.T. Ridgway, ApJS, 167, 81 (2006), arXiv: astro-ph/0608506

[29] O. Herscovici-Schiller, L.M. Mugnier, J.F. Sauvage, MNRAS, 467, L105 (2017), 1701.08633

[30] C. Aime, R. Soummer, ApJ, 612, L85 (2004)

[31] J. Kühn, E. Serabyn, J. Lozi, N. Jovanovic, T. Currie, O. Guyon, T. Kudo, F. Martinache, K. Liewer, G. Singh et al., PASP, 130, 035001 (2018), 1712.02040 
[32] N. Jovanovic, F. Martinache, O. Guyon, C. Clergeon, G. Singh, T. Kudo, V. Garrel, K. Newman, D. Doughty, J. Lozi et al., PASP, 127, 890 (2015), 1507.00017

[33] C. Marois, D. Lafrenière, R. Doyon, B. Macintosh, D. Nadeau, ApJ, 641, 556 (2006), arXiv: astro-ph/0512335

[34] D. Lafrenière, C. Marois, R. Doyon, D. Nadeau, É. Artigau, ApJ, 660, 770 (2007), arXiv: astro-ph/0702697

[35] B. Norris, G. Schworer, P. Tuthill, N. Jovanovic, O. Guyon, P. Stewart, F. Martinache, MNRAS, 447, 2894 (2015)

[36] R. Soummer, L. Pueyo, J. Larkin, ApJL 755, L28 (2012), 1207.4197

[37] F. Martinache, ApJ 724, 464 (2010), 1009. 3933

[38] R.C. Jennison, MNRAS, 118, 276 (1958)

[39] J.E. Baldwin, C.A. Haniff, C.D. Mackay, P.J. Warner, Nature, 320, 595 (1986)

[40] B. Pope, P. Tuthill, S. Hinkley, M.J. Ireland, A. Greenbaum, A. Latyshev, J.D. Monnier, F. Martinache, MNRAS, 455, 1647 (2016), 1510.06406

[41] F. Malbet, J.W. Yu, M. Shao, PASP, 107, 386 (1995), arXiv: astro-ph/9502042

[42] P.J. Bordé, W.A. Traub, ApJ, 638, 488 (2006), arXiv: astro-ph/0510597

[43] A. Give'On, N.J. Kasdin, R.J. Vanderbei, Y. Avitzour, Journal of the Optical Society of America A 23, 1063 (2006)

[44] L. Pueyo, J. Kay, N.J. Kasdin, T. Groff, M. McElwain, A. Give'on, R. Belikov, Appl. Opt.48, 6296 (2009)

[45] R. Galicher, P. Baudoz, G. Rousset, AEAA, 488, L9 (2008), 0807. 2467

[46] M.A. Kenworthy, J.L. Codona, P.M. Hinz, J.R.P. Angel, A. Heinze, S. Sivanandam, ApJ, 660, 762 (2007), astro-ph/0702324

[47] G.P.P.L. Otten, F. Snik, M.A. Kenworthy, M.N. Miskiewicz, M.J. Escuti, Optics Express 22, 30287 (2014), 1412.0660 


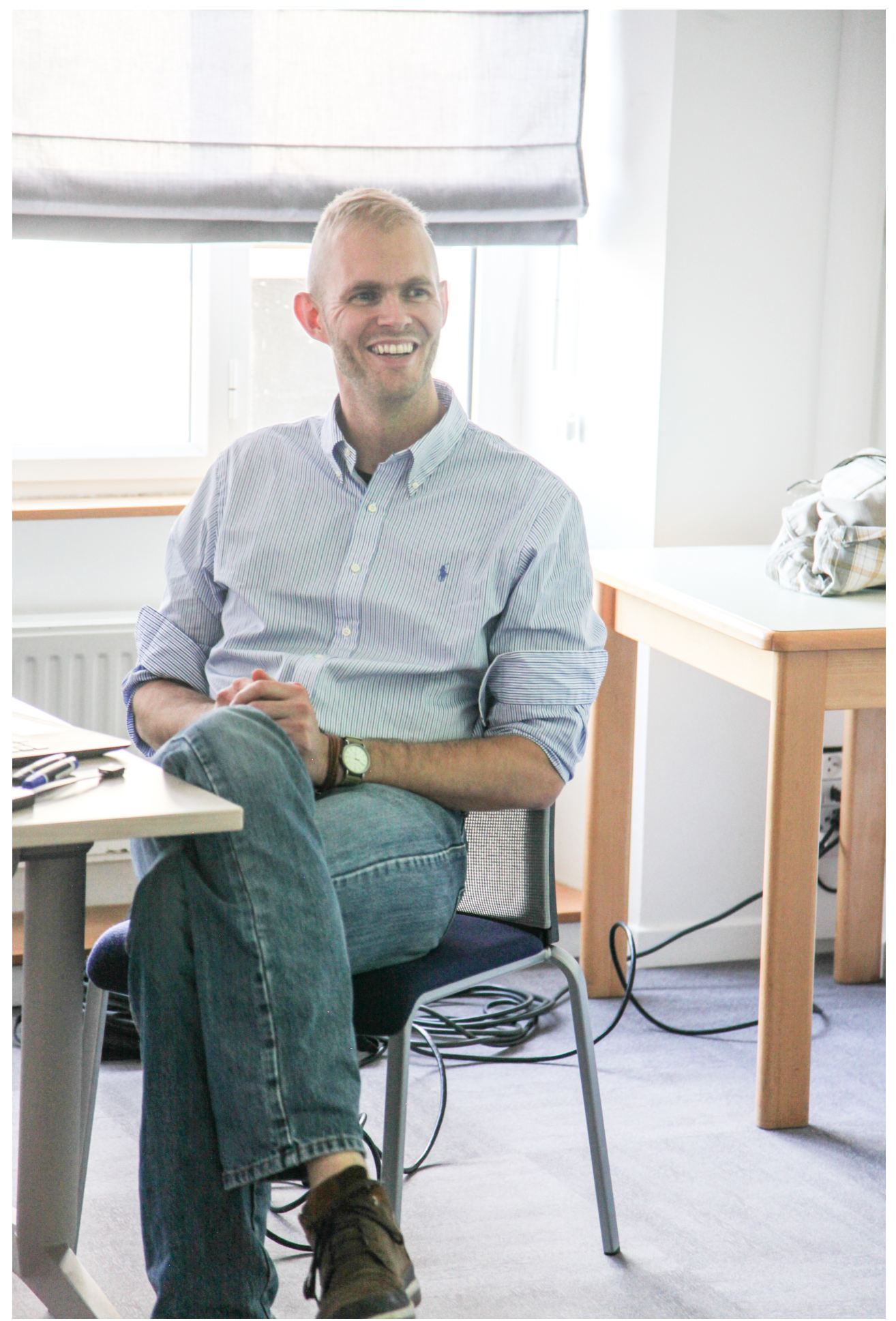

Figure 33. Frantz Martinache, relaxing after his excellent lecture. 


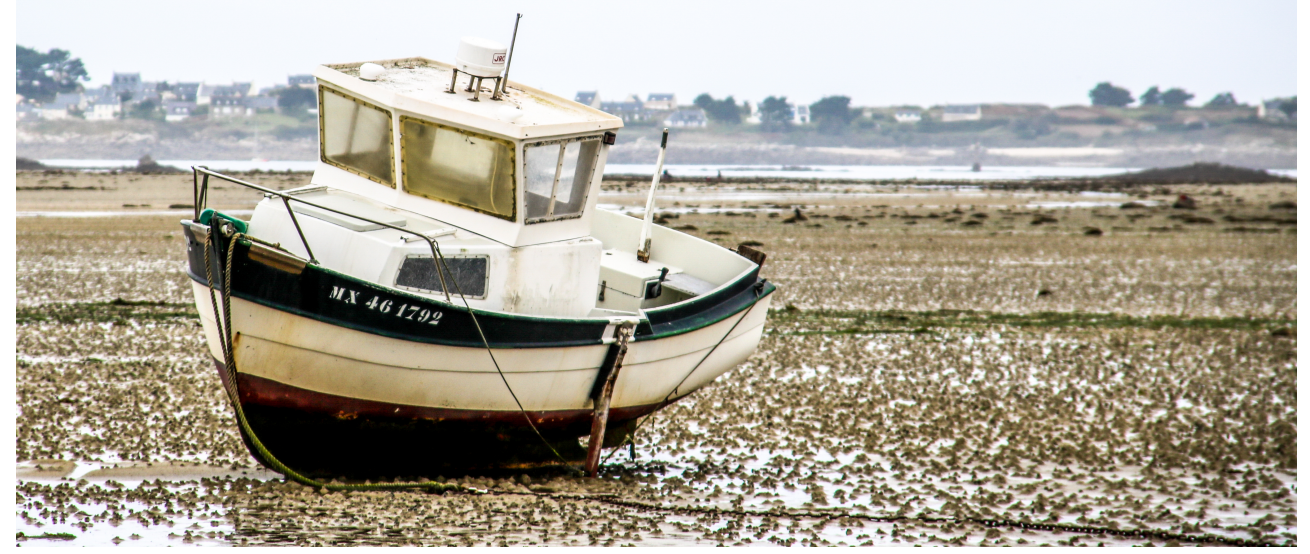

Figure 34. Lonely boat at low tide by the school's hotel.

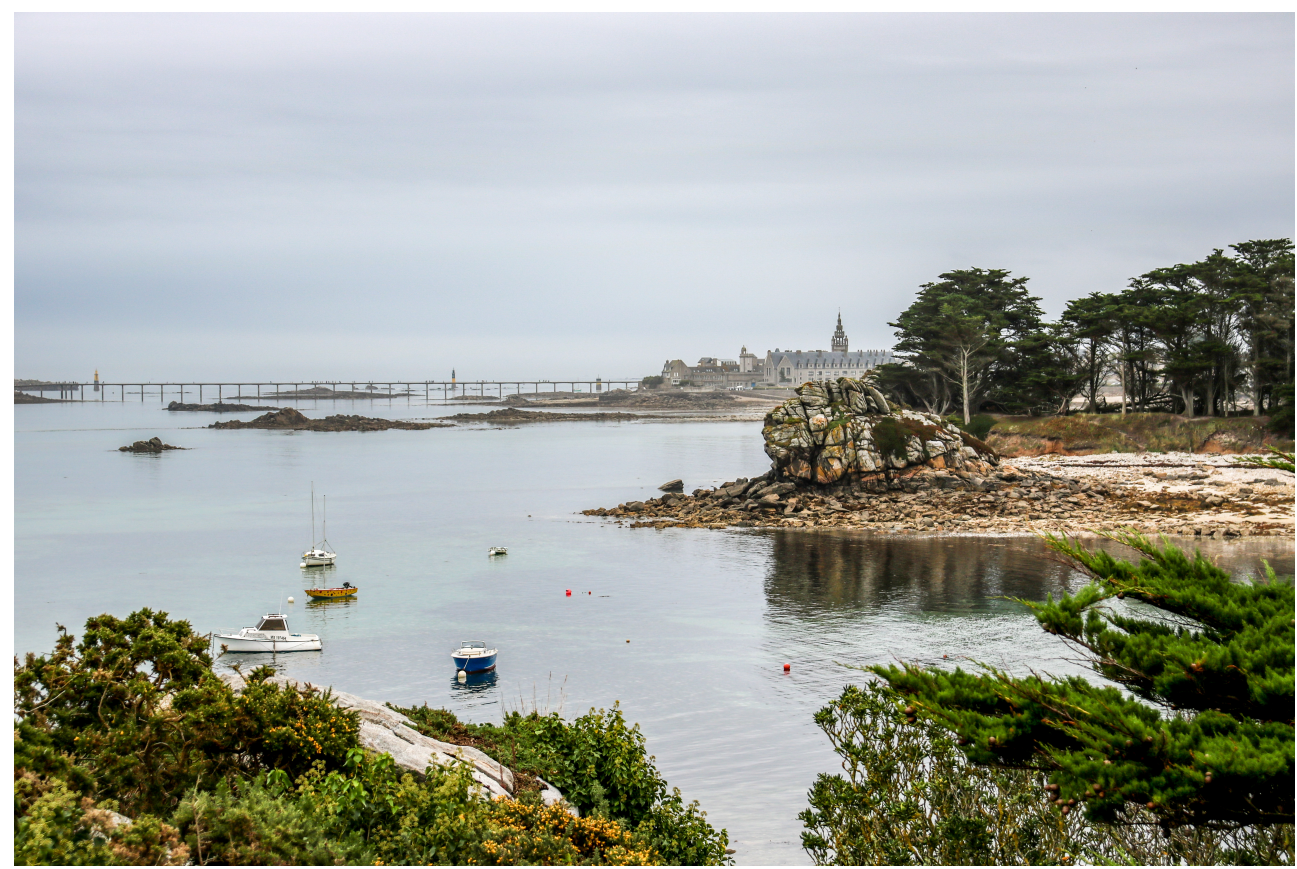

Figure 35. View of the picturesque town of Roscoff, traditional departure point for French Johnnies, selling onions in England: the origin of the cliché for English people, the Frenchmen with onions around their neck. 
Introduction to optical/IR interferometry: history and basic principles 


\title{
Introduction to optical//R interferometry: history and basic principles
}

\author{
Jean Surdej ${ }^{1,3, \ddagger}$ \\ ${ }^{1}$ Institute of Astrophysics and Geophysics, Liège University, Allée du 6 Août 19c, 4000 Liège, Belgium
}

\begin{abstract}
The present notes refer to a 3-hour lecture delivered on 27 September 2017 in Roscoff during the 2017 Evry Schatzman School. It concerns a general introduction to optical/IR interferometry, including a brief history, a presentation of the basic principles, some important theorems and relevant applications. The layout of these lecture notes is as follows. After a short introduction, we proceed with some reminders concerning the representation of a field of electromagnetic radiation. We then present a short history of interferometry, from the first experiment of Fizeau and Stefan to modern optical interferometers. We then discuss the notions of light coherence, including the theorem of Zernickevan Cittert and describe the principle of interferometry using two telescopes. We present some examples of modern interferometers and typical results obtained with these. Finally, we address three important theorems: the fundamental theorem, the convolution theorem and the Wiener-Khinchin theorem which enable to get a better insight into the field of optical/IR interferometry.
\end{abstract}

\section{Introduction}

In the absence of the Earth atmosphere above a ground-based telescope equipped with a mirror having a diameter $D_{1}$, Figure 36 illustrates the image one would observe from a point-like star recorded in the focal plane in monochromatic light at a wavelength $\lambda$. It is a dot of light, the well-known Airy disk, which angular radius measured in radian is simply given by 1.22 $\lambda / D_{1}$. Unfortunately, the Airy disk does not contain any information relative to the star being imaged, irrespective of its size, shape, effective temperature, luminosity, distance, etc. A larger telescope with a diameter $D_{2}>D_{1}$, would similarly lead to a smaller Airy disk (1.22 $\lambda / D_{2}$ ) of light for the star being imaged (see Fig. 37), providing a slightly better angular resolution image but with no more specific information related to the star. While observing an extended celestial source (cf. a distant resolved Earth-like planet as shown in Fig. 38), more details are seen with the telescope having a larger diameter. The dream of astronomers is therefore to construct always larger telescopes but presently there is a limit ( $D \sim 40 \mathrm{~m}$ ) over which it is technologically difficult to construct a single mirror telescope (cf. the ELT, TMT, GMT projects).

Fortunately, in 1868 Fizeau and Stephan just realized that "In terms of angular resolution, two small apertures distant of $B$ are equivalent to a single large aperture of diameter $B$." (see Fig. 39). This is actually the subject of the present lecture: to understand how it is possible to reconstruct high angular resolution images of a distant celestial source using modern optical/IR interferometers such as VLTI, CHARA, etc. In fact, the image of a distant star that

\footnotetext{
†e-mail: jsurdej@ulg.ac.be
} 
one would see in the focal plane of a Fizeau-type interferometer is no longer just an Airy disk due to each single telescope aperture but a brighter Airy disk superimposed with a series of interference fringes, alternately bright and dark, perpendicularly oriented with respect to the line joining the two telescopes and with an inter-fringe angular separation equal to $\lambda / B$, where $B$ is the baseline of the interferometer (see Fig. 40). This naturally leads to the hope that it will be possible to retrieve along the direction of the baseline having a length $B$ an angular resolution that is equivalent to that of a single dish telescope having a diameter $B$. As a summary, figure 41 illustrates the improvement expected in angular resolution while observing an extended celestial source with telescopes of increasing size $\left(D_{2}>D_{1}\right)$ and with an interferometer composed of two telescopes separated by a distance $B>D$.
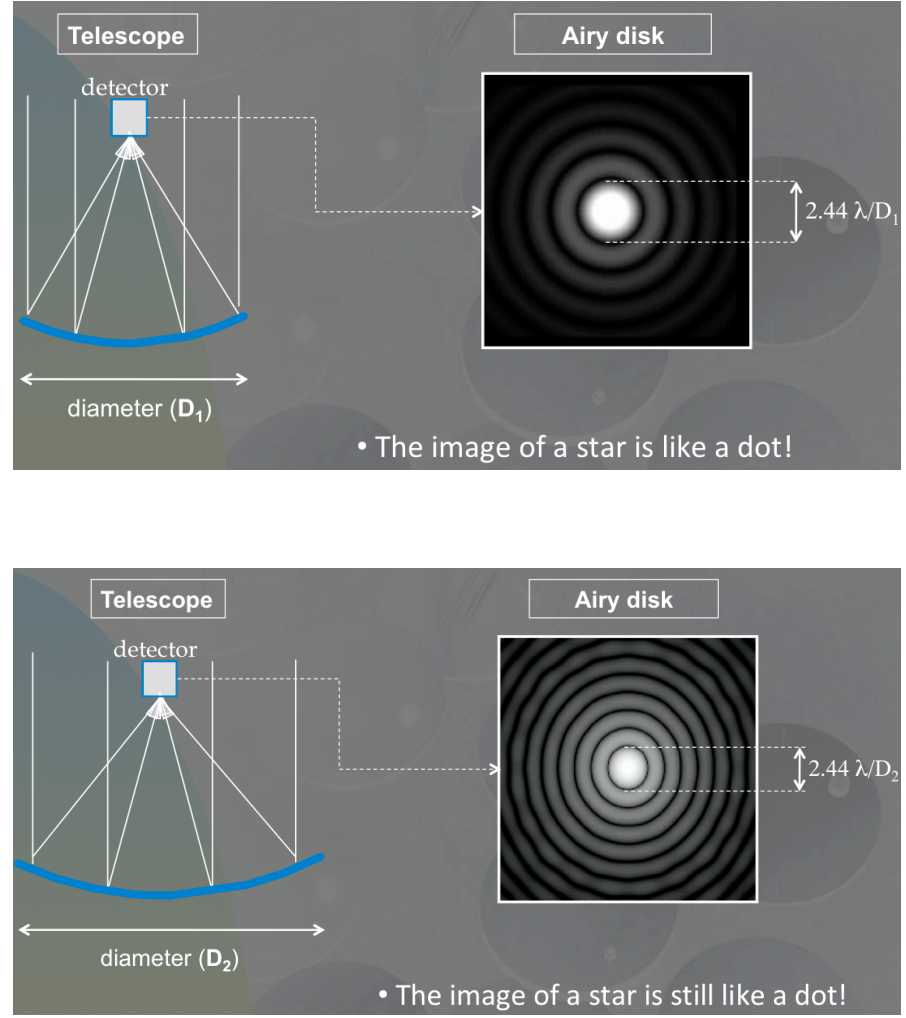

Figure 36. Airy disk of a point-like star recorded in the focal plane of a telescope with diameter $D_{1}$. The angular diameter of the Airy disk is $2.44 \lambda / D_{1}$.

Figure 37. As the diameter of a telescope increases $\left(D_{2}>D_{1}\right)$, the Airy disk of a point-like star gets smaller.

In mathematical terms, the convolution theorem states that the image $\mathrm{I}(\zeta, \eta)$ we observe in the focal plane of an instrument (single dish telescope or interferometer) from a distant extended source as a function of its angular coordinates $\zeta, \eta$ is the convolution product of the real source image (cf. the extended Earth-like planet), $\mathrm{O}(\zeta, \eta)$ by the point spread function $\operatorname{PSF}(\zeta, \eta)$ of the telescope (i.e. the Airy disk, see Fig. 42) or of the interferometer (i.e. the Airy disk crossed by the interference fringes).

While taking the Fourier transform (FT) of the first expression given in Fig. 42, we find that $F T[I(\zeta, \eta)](u, v)$ is simply equal to the natural product of $F T[P S F(\zeta, \eta)](u, v)$ and $F T[O(\zeta, \eta)](u, v)$ where $u, v$ represent the angular space frequencies defined as $u=B_{u} / \lambda$ and $v=B_{v} / \lambda$, respectively, where $B_{u}$ and $B_{v}$ correspond to the projected baselines of the interferometer along the directions parallel to the angles $\zeta, \eta$. One can then expect that by just taking the inverse Fourier transform $F T^{-1}$ of $F T[O(\zeta, \eta)](u, v)$, it will become possible to retrieve high angular resolution information about the extended source with an angular resolution 

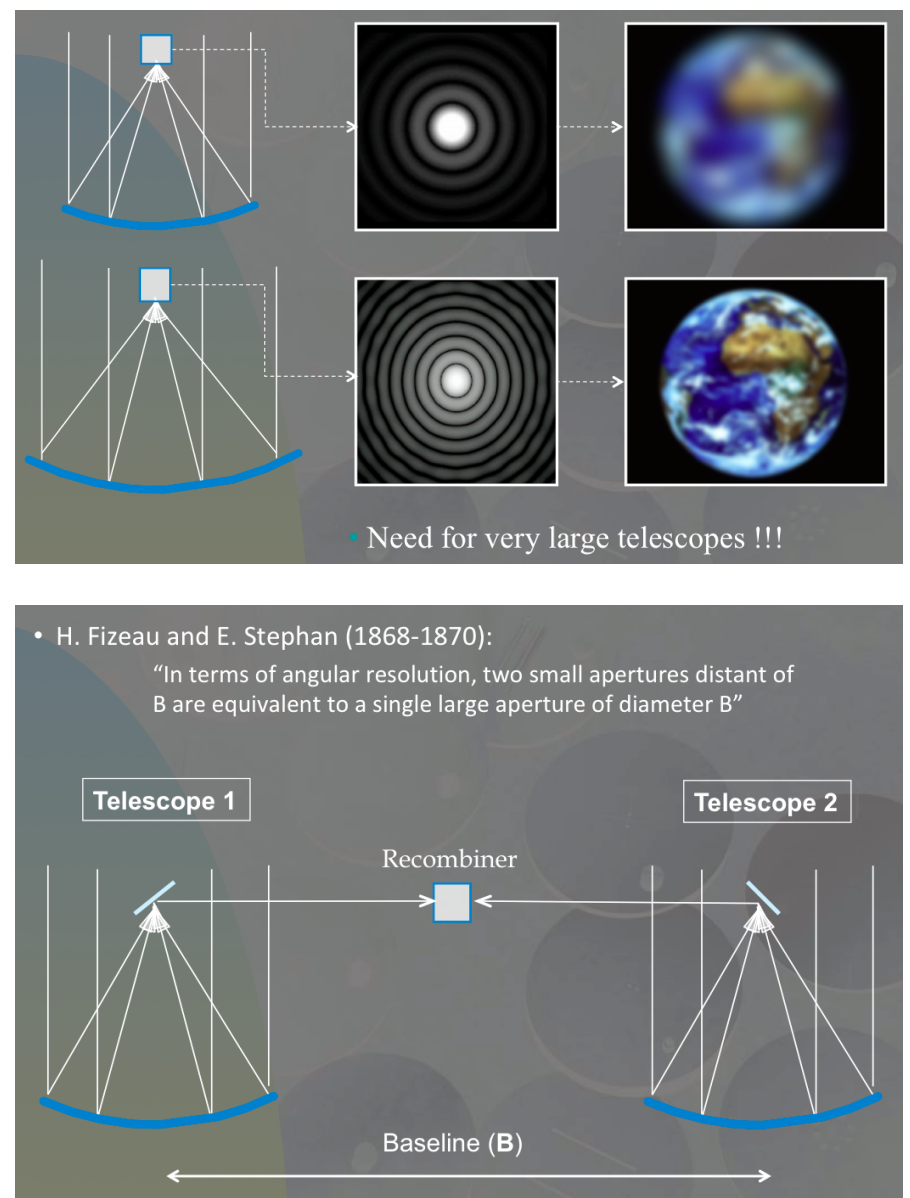

Figure 38. While observing an extended celestial object (cf. an Earth-like planet) above the atmosphere, we see more details as the diameter of the telescope increases.

Figure 39. Fizeau and Stephan proposed to recombine the light from two independent telescopes separated by a baseline $B$ to recover the same angular resolution as that given by a single dish telescope having a diameter $B$.

equivalent to $1 / u=\lambda / B_{u}$ and $1 / v=\lambda / B_{v}$, respectively:

$$
O(\zeta, \eta)=F T^{-1}[F T[O(\zeta, \eta)](u, v)](\zeta, \eta)=F T^{-1}\left[\frac{F T[I(\zeta, \eta)](u, v)}{F T[P S F(\zeta, \eta)](u, v)}\right](\zeta, \eta) .
$$

The quantity $F T[I(\zeta, \eta)](u, v)$ can be directly derived from the observation of the extended source with the optical/IR interferometer while the other quantity $\operatorname{FT}[\operatorname{PS} F(\zeta, \eta)](u, v)$ can be obtained from the observation of a point-like (unresolved) star. During this lecture, we shall see that the Wiener-Khinchin theorem states that the latter quantity is also merely given by the auto-correlation function of the distribution of the complex amplitude of the radiation field in the pupil plane of the observing instrument being used (single dish telescope or interferometer). The goal of the present lecture is to establish relations such as Eq. (47).

\section{Some reminders}

With a few exceptions (cf. the Moon, the Sun, the Andromeda Galaxy, etc.), all the celestial objects that we see in the sky appear to us, with the naked eye, as point-like objects. Apart from their apparent motion with respect to the fixed stars on the celestial sphere, we are not even able to distinguish between the images of Jupiter, Saturn or even Venus from those of ordinary stars. We describe in this course an observation method based on the principle of a 


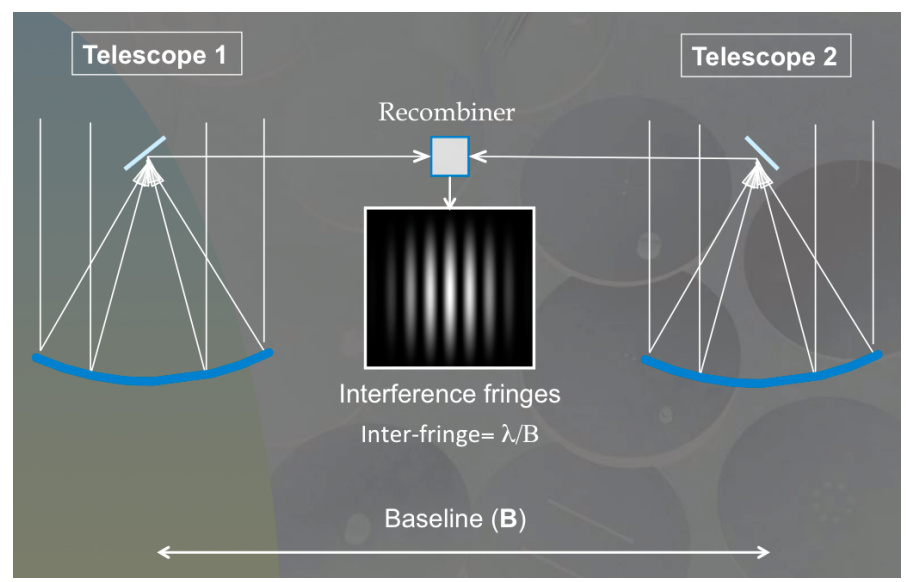

Figure 40. When recombining the monochromatic light of two independent telescopes, there results the formation of a pattern of bright and dark fringes superimposed over the combined Airy disk. The angular inter-fringe separation is equal to $\lambda / B$.

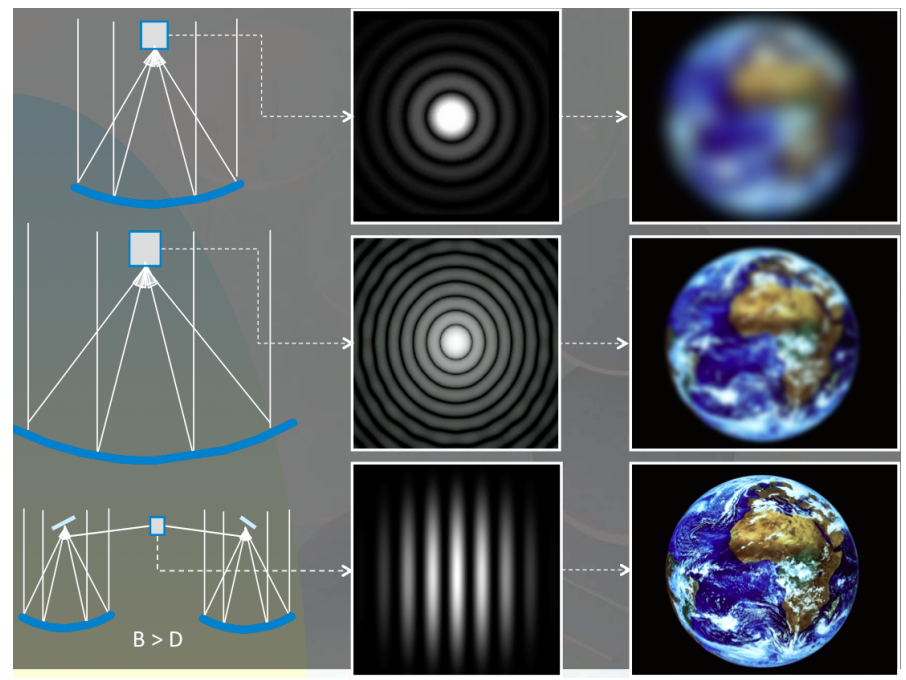

Figure 41. Improvement expected in angular resolution while observing an extended celestial source (cf. an Earth-like planet) with telescopes of increasing size $\left(D_{2}>D_{1}\right)$ and with an interferometer composed of two telescopes separated by a baseline $B>D$.

Fizeau-type interferometer, which allows with just some basic cooking equipment to resolve angularly a planet such as Venus, when it is at its maximum apparent brightness $(V \sim-4.4)$.

If we assimilate for a moment the disc of a star, or even that of Venus, to the filament of a light bulb, the object of the present lecture can still be formulated as follows: given a common electric light bulb inside which is a filament, having a certain thickness $T$ (measured perpendicularly to the line-of-sight) and which is incandescent (cf. a star), how to measure the thickness $T$ of this filament (diameter of the star) not only without breaking the bulb but also assuming that it is so far away from us, at a distance $z$, that it is not possible for us to angularly resolve the filament with the naked eye (see Fig. 43a)?

Let us now recall that knowledge of the angular radius $(\rho=R / z)$ of a star located at a distance $z(z>>R)$ and having a linear radius $R$ allows the direct determination of its flux $F$ at the stellar surface from the flux $f$ observed on Earth (as a reminder $F=f / \rho^{2}$, see Fig. 43b). If we can measure the absolute distance $z$ of the star, we can also determine its linear radius $R$ from its angular radius $\rho(R=\rho z)$. Moreover, knowledge of the intrinsic flux $F$ of the star allows an immediate determination of its effective temperature $T_{\text {eff }}$, thanks to the application of the Stefan-Boltzmann law $\left(F=\sigma T_{e f f}^{4}\right)$. It then results that $T_{e f f}=\left(f / \sigma \rho^{2}\right)^{1 / 4}$. The measurements of the angular radius and of the flux of a star measured on Earth thus lead to the 


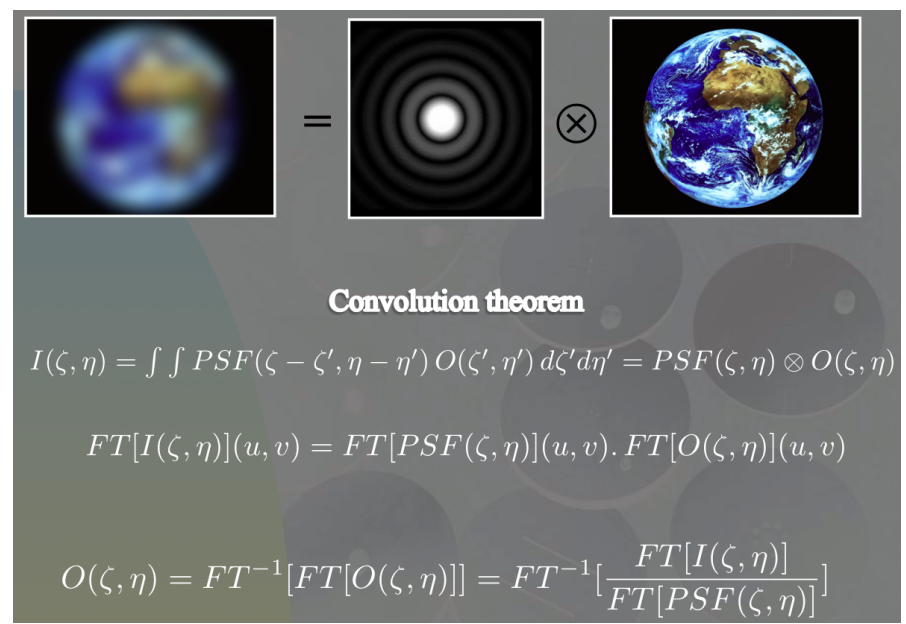

(a)

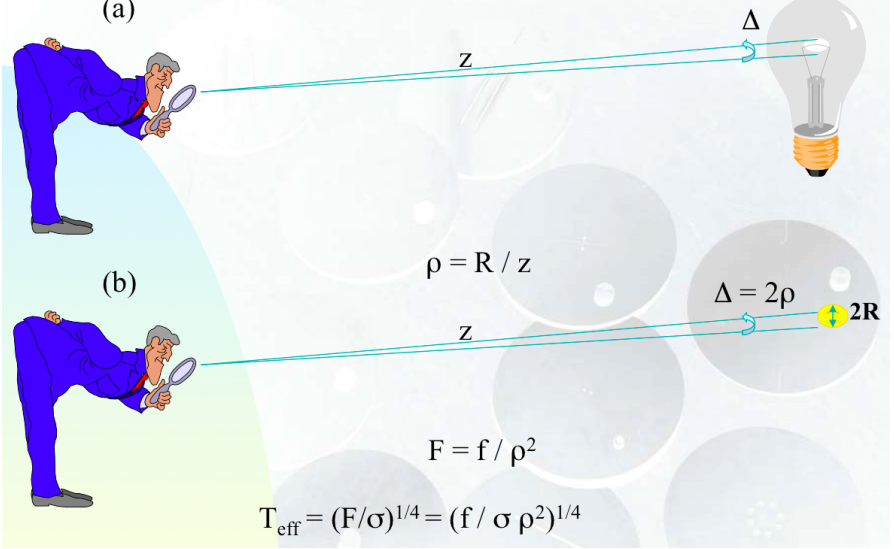

Figure 42. The image $\mathrm{I}(\zeta, \eta)$ we observe in the focal plane of an instrument (cf. single dish telescope) from a distant extended source as a function of its angular coordinates $\zeta, \eta$ is the convolution product of the real source image (cf. the extended Earth-like planet, $\mathrm{O}(\zeta, \eta))$ by the point spread function $\operatorname{PSF}(\zeta, \eta)$ of the telescope.
Figure 43. Resolving the angular diameter of a star (b) is alike trying to estimate the angular size of the filament of a light bulb (a).

determination of the effective temperature $T_{\text {eff }}$ of that star. As a reminder, this temperature is directly involved in the construction of stellar atmosphere models and stellar evolution. We will also show that Fizeau-type stellar interferometry literally allows direct imaging with very high angular resolution of distant bodies by the method of aperture synthesis. Let us now proceed with a few theoretical reminders about the description of a field of electromagnetic light radiation.

\subsection{Complex representation of an electromagnetic wave}

Let us first remind that a beam of light radiation can be assimilated to the propagation of a multitude of electromagnetic waves at the speed of $299,792 \mathrm{~km} \mathrm{~s}^{-1}$ in the vacuum. If, for the sake of simplicity, we assume that we deal with a plane monochromatic wave, linearly polarized, propagating along the direction of abscissa $z$, the electric field $E$ at any point in space and at time $t$, can be represented by a sinusoidal type function taking for example the shape

$$
E=a \cos (2 \pi(v t-z / \lambda))
$$

where 


$$
\lambda=c T=c / v
$$

$c, \lambda, v, T$ and $a$ representing the speed of light, the wavelength, the frequency, the period and the amplitude of the electromagnetic vibrations, respectively (see Figure 44).

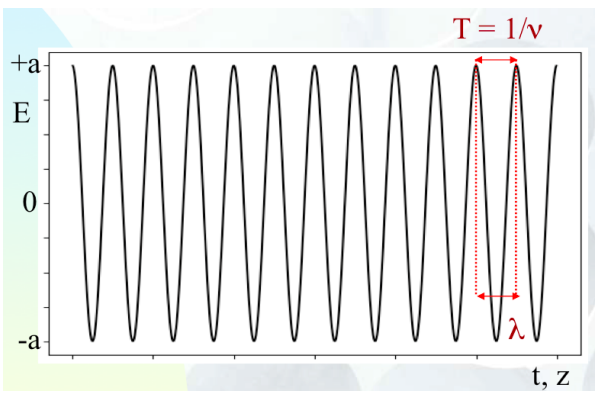

Figure 44. Representation of an electromagnetic wave.

We know how convenient it is to rewrite the previous equation in complex notation:

$$
E=\operatorname{Re}\{a \exp [i 2 \pi(v t-z / \lambda)]\}
$$

where $R e$ represents the real part of the expression between the two curly braces. This complex representation of an electromagnetic wave has the great advantage that the exponential function can now be expressed as the product of two functions depending separately on the spatial and temporal coordinates

$$
E=a \exp (-i \phi) \exp (i 2 \pi v t)
$$

where

$$
\phi=2 \pi z / \lambda
$$

If we suppose that all the operations that we carry out on the electric field $E$ are linear, it is of course very convenient to use in our calculations its complex representation (see Eq.(51)) and to take at the end the real part of the result obtained.

We can then rewrite the previous equation as follows:

$$
E=A \exp (i 2 \pi v t)
$$

where

$$
A=a \exp (-i \phi)
$$

with A representing the complex amplitude of the vibration.

Because of the extremely high frequencies of electromagnetic waves corresponding to visible radiations $\left(v \sim 610^{14} \mathrm{~Hz}\right.$ for $\lambda=5000 \AA$ ), we recall that it is not normally possible to make direct observations of the electric field $E$ (the situation is different in the radio domain). The only measurable quantity is the intensity $I$, which is the time average of the amount of energy passing through a unit surface element, per unit of time and solid angle, placed perpendicularly to the direction of propagation of the light beam.

The intensity $I$ is therefore proportional to the temporal average of the square of the electric field:

$$
\left\langle E^{2}\right\rangle=\lim _{T \rightarrow \infty} \frac{1}{2 T} \int_{-T}^{+T} E^{2} d t
$$


which is reduced to (e.g. replace in the previous relation $E$ by Eq.(48))

$$
\left\langle E^{2}\right\rangle=\frac{a^{2}}{2}
$$

where $a$ is the real amplitude of the electric field.

By convention, the intensity of the radiation is defined by the following relation:

$$
I=A A^{*}=|A|^{2}=a^{2} .
$$

\subsection{Principle of Huygens-Fresnel}

We recall that, according to Huygens, each point of a wavefront can be considered as being the centre of a secondary wave leading to the formation of spherical wavelets, and that the main wavefront, at any subsequent moment can be considered as the envelope of all these wavelets (see Fig. 45).

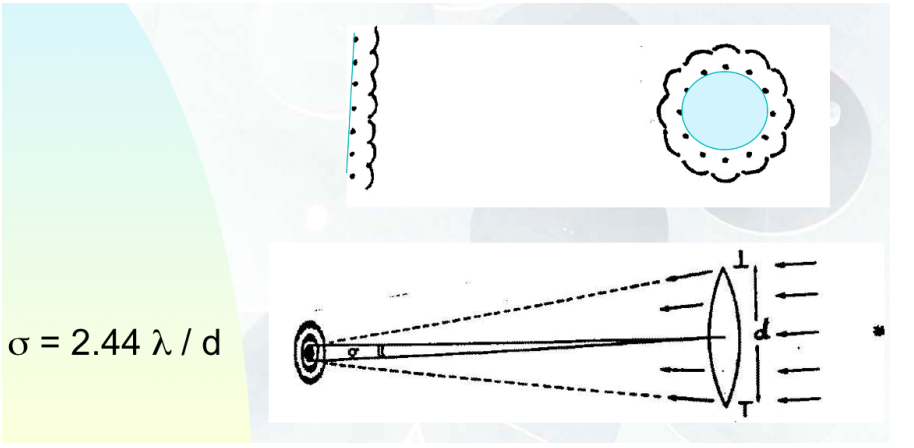

Figure 45. Illustration of the Huygens-Fresnel principle during the propagation of a plane or circular wavefront and diffraction of light which encounters a converging lens.

Using this model, Fresnel was the first to account for the observed effects of light diffraction, assuming that secondary wavelets interfere with each other. This combination of the Huygens construction method and the Fresnel interference principle is called the HuygensFresnel principle. This is the basis of the concept of the Fourier transform. Let us remind a direct application of this principle when studying the formation of the image of a distant object at the focus of a telescope having a linear diameter $d$. Following the diffraction of the waves at the passage of the opening of the telescope (as if the waves were trying to spread and bypass the obstacles), we observe a phenomenon of redistribution of the energy of the light wave: the image of a point-like source produced by a converging circular objective (lens or mirror) is not a point but spreads in a diffraction pattern called the "Airy disk" (see Fig. 45). The angular diameter of the central spot is (in radian):

$$
\sigma=2.44 \lambda / d
$$

where $\lambda$ is the wavelength of light and $d$ is the linear diameter of the aperture.

We can resolve an extended source by direct imaging, if and only if, its angular diameter $\Delta$ $(=2 \rho)$ is somewhat larger than $\sigma$. For example, our pupil whose approximate diameter varies between 1 and $5 \mathrm{~mm}$, allows us to angularly resolve nearby objects separated by more than 138 " and 28", respectively. In the visible range, a telescope, with a diameter of $14 \mathrm{~cm}$, will allow us to resolve objects with an angular dimension larger than 1", and for diameters larger than $14 \mathrm{~cm}$, their collecting area will naturally be enhanced but their angular resolution will remain limited to (more or less) 1" because of the atmospheric agitation (see Fig. 46). In fact, under the influence of temperature and pressure gradients, a regime of eddies establish itself 
in the Earth atmosphere which, at low altitude $(\sim 10 \mathrm{~km})$, have dimensions of the order of 20 $\mathrm{cm}$ (sometimes only a few $\mathrm{cm}$, sometimes 30 or $40 \mathrm{~cm}$ ) and evolution periods of the order of a few milliseconds. Optically, these changes manifest themselves by an inhomogeneity in the refractive index distribution. The amplitude and the relative phase shift of the electromagnetic field in the pupil plane thus get disturbed in a random manner.

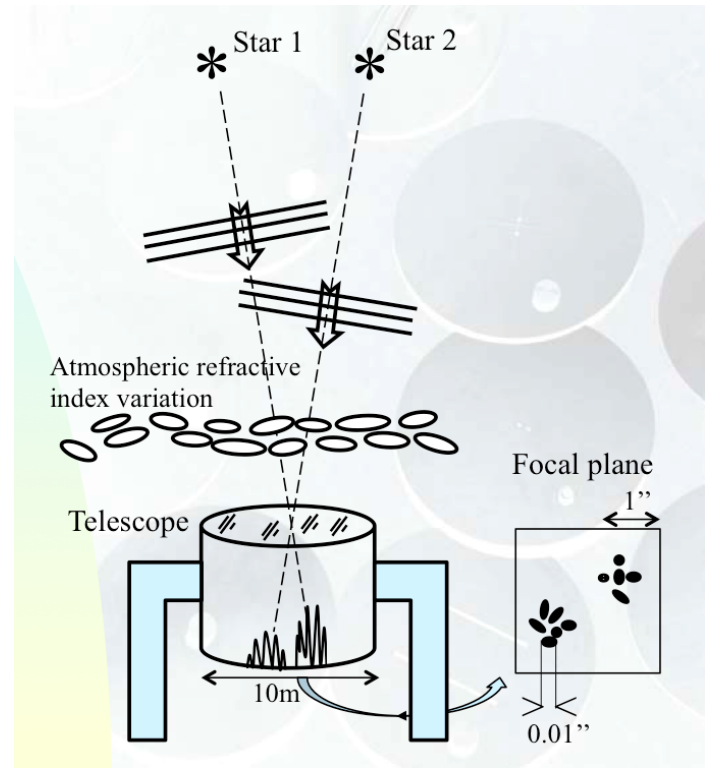

Figure 46. Atmospheric agitation above the objective of a large telescope causing the seeing effects seen in its focal plane.

It follows that if we observe the Moon, Jupiter, etc. either with the largest telescope in the world with a diameter of $10 \mathrm{~m}(\sigma=0.014$ ") or with an amateur telescope with a diameter of $14 \mathrm{~cm}(\sigma=1$ "), we will see the same details under good seeing conditions. The brightness will of course be larger with the $10 \mathrm{~m}$ telescope ... but probably too bright for the eye not to be blinded by the image of the Moon or Jupiter.

The diffusion indicator of the atmosphere is defined as being the average inclination perturbation of the wave surfaces. This tilt disturbance reaches values that vary between 1" and 10 ", depending on the site and the moment. This phenomenon is detected differently according to the dimensions of the instrument used. The eye, which has an angular resolution close to the minute of arc, will be sensitive only to variations of amplitude: we then see stars flickering. An instrument of 10 to $20 \mathrm{~cm}$ in diameter will detect tilt variations and the focal image will oscillate around an average position. For larger instruments, a large number of eddies will, at the same time, be involved in the formation of the focal image. This will therefore have the dimensions of the diffusion indicator of the atmosphere. The spatial coherence of the entrance pupil will allow, for a point-like source, the realization of interference phenomena between the radiations passing through different points of the pupil. A statistical study makes it possible to show that the resulting focal image, delimited by the diffusion indicator, consists of a set of granules (called 'speckles') which have the size of the Airy disk of the instrument (see Fig. 46). These granules swarm in the diffusion spot at the rhythm of the change of the atmospheric eddies. The stability of the focal image is therefore also of the order of the millisecond. The technique of speckle interferometry, developed by the French astronomer Antoine Labeyrie, allows to re-construct the images of the stars observed with the angular resolution given by the true diameter of the telescope. 


\section{Brief history about the measurements of stellar diameters}

In the past, there have been numerous attempts to measure angular diameters of stars, and we will first recall three of these approaches that clearly show the difficulties encountered.

\subsection{Galileo}

A first experimental attempt to measure the angular diameter of stars was made by Galileo (1632). He proceeded as follows: placing himself behind a rigid wire (whose thickness $D$ was known, see Fig. 47) suspended vertically, he determined the distance $z$ to which he had to move in so that the image of the star Vega ( $\alpha$ Lyrae) of magnitude zero got completely obscured by the wire. Galileo deduced that the angular diameter of Vega, equal to that of the wire, was about 5", which was in itself a rather revolutionary result, since the value adopted at that epoch for the angular diameter of the stars was close to 2'. As we saw earlier, the value of 2 ' is certainly the result of the low angular resolution of our eye, while the 5" angular diameter measured by Galileo was the result of the effects of the atmospheric agitation (seeing effects) at the time of his observations.

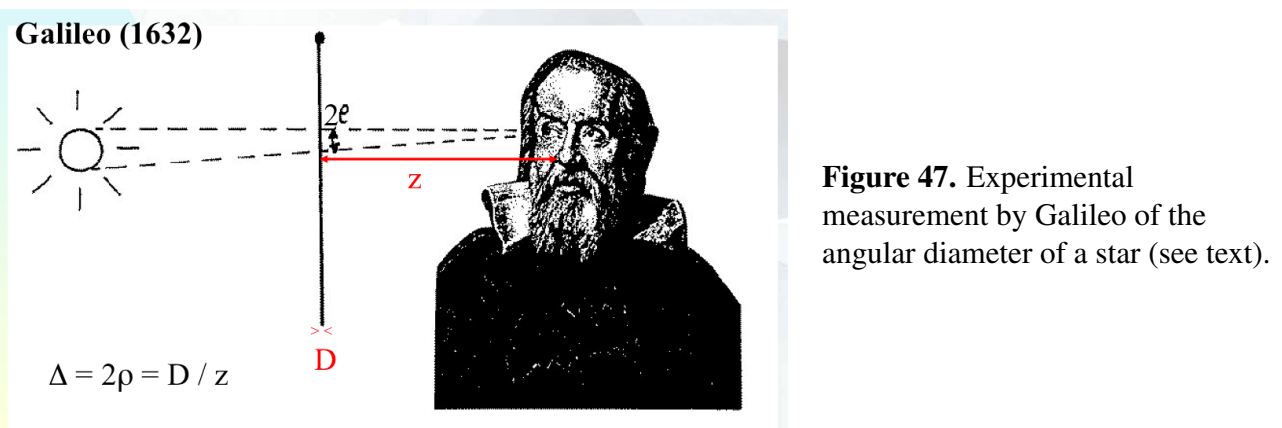

\subsection{Newton}

A theoretical estimate of the angular dimension of a star of magnitude zero was performed by Newton. His approach was as follows: if we suppose that the Sun is a star similar to the stars situated on the celestial sphere and if we place our star of the day at a distance $z$ such that its apparent brightness $V_{\odot}$ becomes comparable to that of a star of magnitude equal to zero, then its angular diameter $\Delta$ should be of the order of $210^{-3}$ " (with the current value of the visual apparent magnitude of the Sun, $V_{\odot}=-26.7$, we find $\sim 810-3$ "). It should be noted that the value currently established for the star Vega with modern interferometers is $310^{-3}$ ". The formula to be used to establish this result can be obtained as follows: the angular diameter of the Sun $\Delta$ placed at the distance of Vega $(V=0)$ is given by the product of the apparent angular diameter of the Sun $\Delta_{\odot}$ times the factor $10^{V_{\odot} / 5}$. As a reminder, the apparent diameter of the Sun is about 30 '.

\subsection{Fizeau-type interferometry}

The third experimental attempt of measuring stellar diameters, based on Fizeau-type interferometry, is in fact the work of prominent scientists such as Young, Fizeau, Stephan, Michelson and Pease. These last two having measured the first angular diameter of a star in 1920. Although other methods of interferometric measurements of stellar angular diameters appeared 
later (cf. the interferometry in intensity of Brown and Twiss in 1957, speckle interferometry by Antoine Labeyrie in 1970, etc.), we will only describe in detail the Fizeau-type interferometry, which is still the most powerful technique used and the most promising measurement of angular diameters of stars and imagery at very high angular resolution of distant bodies by the aperture synthesis method.

Let us first remind the results obtained in the Young double hole experiment (1803, see Fig. 48).

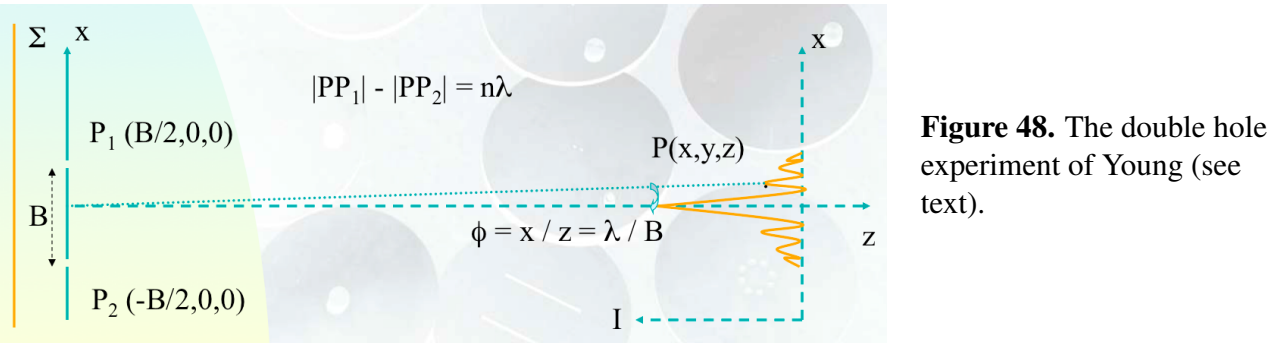

A monochromatic plane wave coming from a distant point-like source is falling on a screen drilled with two holes $\left(P_{1}\right.$ and $\left.P_{2}\right)$ separated along the $x$ axis by a baseline $B$. In accordance with the Huygens-Fresnel principle, the two holes will emit spherical waves that will interfere constructively whenever the difference in their propagation lengths is a multiple of $\lambda$ (see Eq. (59) below), and destructively if it corresponds to an odd number of half wavelengths.

The locus of points $P(x, y, z)$ with cartesian coordinates $x, y, z$ (see Fig. 48) where there will be a constructive interference is thus given by

$$
\left|P_{1} P\right|-\left|P_{2} P\right|=n \lambda
$$

with $n=0, \pm 1, \pm 2$, etc.

Let the points $P_{i}\left(x_{i}, y_{i}, 0\right)$ in the screen plane and $P(x, y, z)$ in the observer plane be such that $\left|x_{i}\right|,\left|y_{i}\right|,|x|,|y|<<|z|$. We then find that

$$
\left|P_{i} P\right|=\sqrt{\left(x-x_{i}\right)^{2}+\left(y-y_{i}\right)^{2}+z^{2}}
$$

which can be simplified at first order (given the above conditions) as follows:

$$
\left|P_{i} P\right|=z\left\{1+\frac{\left(x-x_{i}\right)^{2}+\left(y-y_{i}\right)^{2}}{2 z^{2}}\right\}
$$

Considering the two points $P_{1}$ and $P_{2}$ in the Young's screen, Eq. (59) reduces to

$$
z\left\{1+\frac{\left.(x+B / 2)^{2}+y^{2}\right)}{2 z^{2}}\right\}-z\left\{1+\frac{(x-B / 2)^{2}+y^{2}}{2 z^{2}}\right\}=n \lambda
$$

and finally

$$
\frac{x B}{z}=n \lambda
$$

or else

$$
\Phi=\frac{x}{z}=n \frac{\lambda}{B} .
$$

Since the angular separation $\Phi$ between two successive maxima (or minima) does not depend on the coordinate $y$, there results a pattern of bright and dark fringes, oriented perpendicularly 
with respect to the line joining the two holes, and with an inter-fringe angular separation $\Phi=\lambda / B$. In case the two holes are not infinitely small, the observed interference pattern will naturally overlap the combined Airy disks produced by each single hole. For $\lambda=5500 \AA$ and $B=1 \mathrm{~mm}$, we find that $\Phi=113$ ", just at the limit of our eye visual resolution. In 1868, the French optician Hyppolite Fizeau realized that in the Young's hole experiment presented above, the contrast of the interference fringes decreased as the diameter of the light source widened. Similarly, it decreased when the distance $B$ between the two holes was extended. Was there a simple relation between the angular diameter $\Delta$ of the source and the spacing $B$ between the two holes corresponding to the disappearance of the fringes? Before establishing such a rigorous relationship, let us try to understand this observation intuitively on the basis of simple geometrical considerations (see Fig. 49). Indeed, if instead of considering the diffraction pattern given by Young's holes for a single point-like source, we consider a composite source made of two incoherent point-like sources separated by an angle $\Delta$, that is to say between which there is no interference between their light, it will result in the plane of the observer a superposition of two systems of Young fringes, separated by an angle $\Delta$. If $\Delta \sim \Phi / 2$, there will result a total scrambling of the fringes. The bright fringes of one source will overlap the dark fringes of the second one and their contrast will totally vanish.

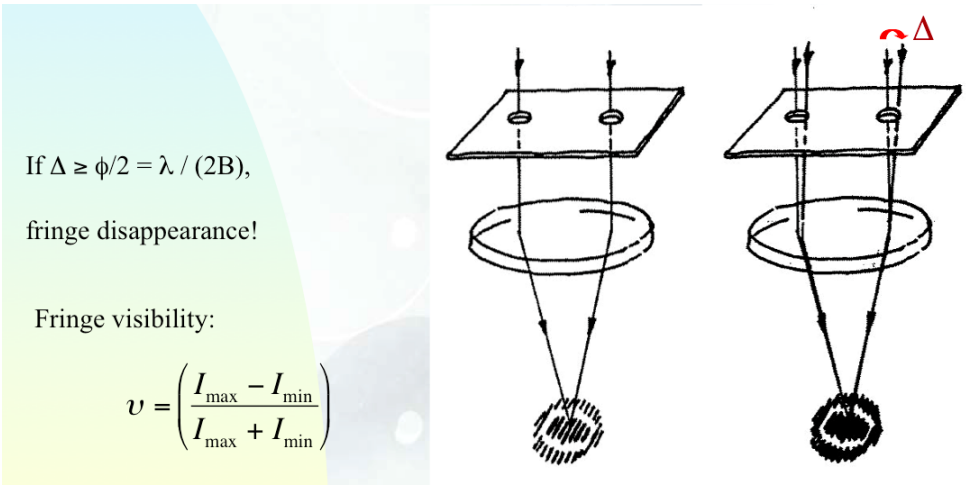

Figure 49. Fizeau experiment: for the case of a single star (left drawing) and for the case of a double star with an angular separation $\Delta$ (right drawing, see text).

From Fig. 49, it is clear that the visibility of the fringes will significantly decrease whenever the following condition takes place

$$
\Delta>\frac{\Phi}{2}=\frac{\lambda}{2 B} .
$$

A quantity that objectively measures the contrast of the fringes is called the visibility. It is defined by the following expression:

$$
v=\left(\frac{I_{\max }-I_{\min }}{I_{\max }+I_{\min }}\right) .
$$

Whenever a star is not resolved, we have $I_{\min }=0$, and thus the visibility $v=1$. If the star is being resolved, $I_{\max }=I_{\min }$ and thus the resulting visibility $v=0$.

Fizeau proposed in 1868 to apply this method to stellar sources. He found sufficient to place a screen drilled with two elongated apertures at the entrance of a telescope pointed 
towards a star and to look in the focal plane by means of a very powerful eyepiece the Airy disk crossed by the Young's fringes and to increase the distance between the two apertures until the visibility of the fringes vanishes.

This experiment is attempted in 1873 by Stephan with the $80 \mathrm{~cm}$ telescope of the Marseille Observatory. All the bright stars visible in the sky are observed. The two openings at the entrance were actually in the form of crescents but one may demonstrate that the contrast of the fringes is independent of the shape of the two openings if they are identical. The result was disappointing: with a maximum base separation of $65 \mathrm{~cm}$ between the openings, no attenuation of the contrast of the fringes was observed for any star. This proved that no star could be resolved using that instrument. Stephan concluded that the angular diameter of the stars is much smaller than 0.16" (see Fig. 50). From these observations, it is of course possible to set a lower limit on the effective temperature $T_{\text {eff }}$ of all those stars (see Section 2). Figure 51 illustrates the $80 \mathrm{~cm}$ Marseille telescope used by Stephan and Fizeau.
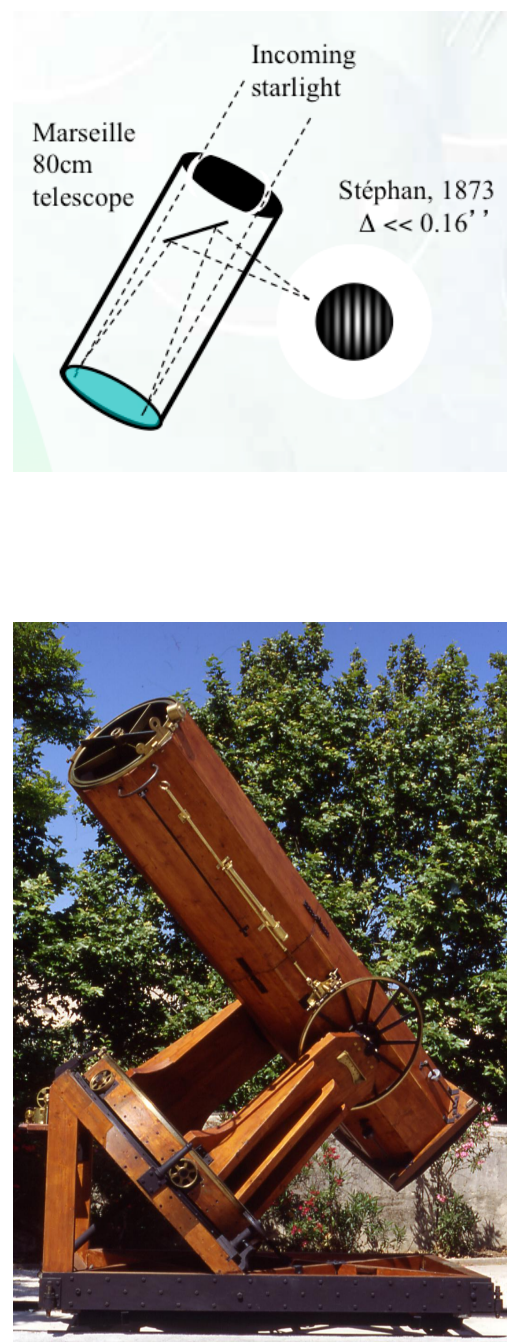

Figure 50. Diagram illustrating the way Fizeau and Stephan proceeded in order to measure the angular diameters of stars with the interferometric technique.
Figure 51. The $80 \mathrm{~cm}$ Marseille telescope used by Fizeau and Stephan. (C) Michel Marcelin. 


\subsection{Home experiments: visualization of the Airy disk and the Young interference fringes}

We propose hereafter two simple experiments that can be carried out at home in order to visualize with our own eyes the Airy disk and the interference fringe patterns. To do this, take a rectangular piece of cartoon $(\sim 5 \mathrm{~cm} \mathrm{x} 10 \mathrm{~cm})$ and fold it in the middle (see Fig. 52). Perforate through the two sides of this piece of cartoon two well separated circular holes having an approximate diameter of $1 / 2 \mathrm{~cm}$. Cut a thin sheet of aluminium paper into two small squares $(\mathrm{cf} .1 \mathrm{~cm} \times 1 \mathrm{~cm}$ ). In one of the two squares, drill with a thin metallic pin a $0.5 \mathrm{~mm}$ (or smaller) diameter circular hole near its centre. Glue inside the folded cartoon the first aluminium piece in such a way that the very small hole is centered with respect to one of the big holes (cf. the lower one) of the cartoon.

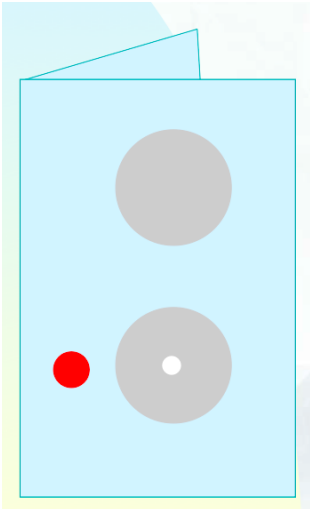

1st experiment!

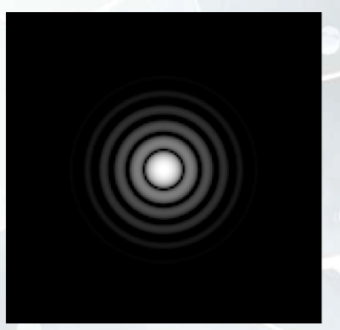

2nd experiment!

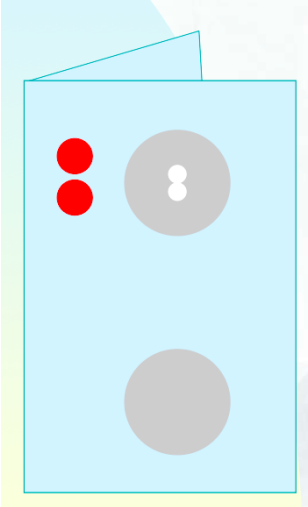

Figure 52. The one hole screen experiment: the small circular hole drilled in the aluminium paper is visible inside the lower bigger hole perforated in the cartoon screen. When looking through this hole at a distant light bulb, you perceive a nice Airy disk (cf. right image).

Take the second square of aluminium and drill with the thin metallic pin two $0.5 \mathrm{~mm}$ (or smaller) circular holes near its centre separated by about $0.5-1 \mathrm{~mm}$ (see Fig. 53). Glue now near the second circular hole (cf. the upper one) inside the folded cartoon this second aluminium square. After, you should glue the two sides of the cartoon in such a way that you can hold it with ease in one hand. Of course you can do this on a single cartoon or on two separate ones. Place at a distance of about $10 \mathrm{~m}$ a small light bulb (cf. the light of a cell phone) and look at it through the single hole drilled in the aluminium. You should see a nice Airy disk which angular diameter is $2.44 \lambda / D$, with $D$ being about $0.5 \mathrm{~mm}$ and $\lambda$ the wavelength of the ambient light $(\sim 5000 \AA)$. Now looking at the distant light source 
through the double hole drilled in the other square of aluminium, you should see an Airy disk superimposed by a series of white and dark fringes, oriented perpendicularly with respect to the line joining the two holes, with an angular inter-fringe separation $\lambda / D$, where $D \sim$ $0.5-1 \mathrm{~mm}$. If you rotate azimuthally the screen, you will observe that the fringes also rotate since they constantly remain perpendicular to the line joining the two small holes of the milliinterferometer. While getting closer to the small light bulb, you will notice that the visibility of the fringes decreases. Let Dist be the distance around which the latter totally vanishes. The product of Dist by $\lambda / D$ corresponds to the linear diameter of the light bulb. Instead of changing the distance between the light bulb and the milli-interferometer, one could change the separation between the two holes and determine the separation for which the interference fringes disappear. Adopting the same approach as Stephan, Abraham Michelson used in 1890 the Lick $30 \mathrm{~cm}$ telescope to resolve the four Galilean satellites of Jupiter. Their angular sizes were of the order of 0.8 " - 1.4 " while the resolving power provided by the largest baseline he used was about 0.5 ". An excellent agreement was found for the angular diameters of the satellites with the classical measurements made at the same time. To resolve the biggest stars, much longer baselines are needed. Michelson and Pease built a $7 \mathrm{~m}$ metal beam carrying four $15 \mathrm{~cm}$ flat mirrors that they installed at the top of the Mount Wilson telescope, having a diameter of $2.5 \mathrm{~m}$ (see Fig. 54).

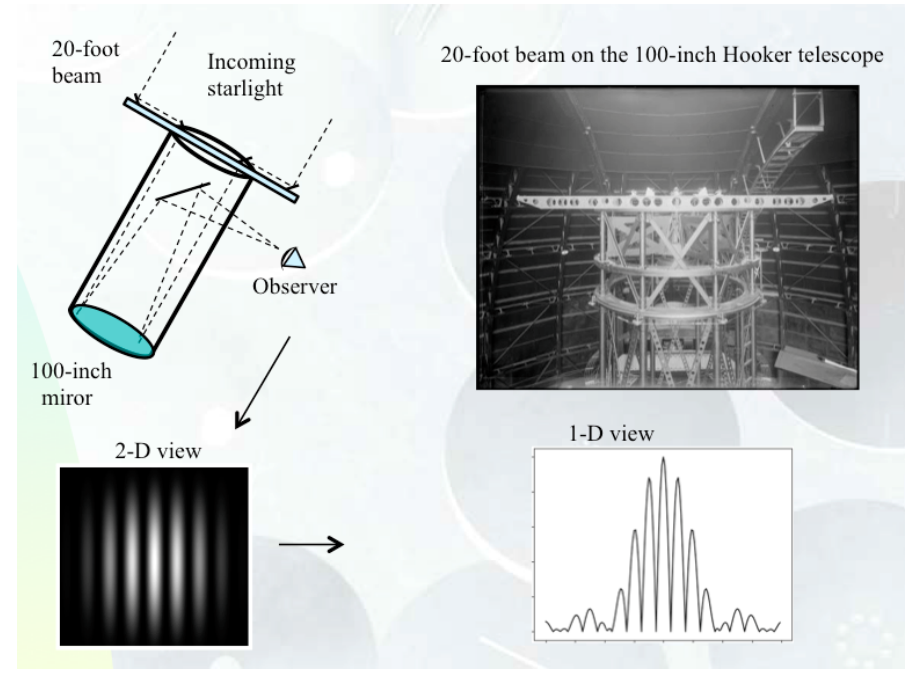

Figure 54. The stellar interferometer of Michelson and Pease set on top of the $2.5 \mathrm{~m}$ Mount Wilson telescope. (C) The Observatories of the Carnegie Institution.

The two mobile exterior mirrors formed the basis of the interferometer and the two fixed interior mirrors returned the star's light into the telescope. With a maximum baseline of $7 \mathrm{~m}$, the smallest measurable angular diameter was 0.02". Use of this first stellar interferometer was very delicate because the visualization of the Young fringes was only possible if the two optical paths from the star passing through the two exterior mirrors and reaching the observer eyes were equal to an accuracy of about 2 microns (see discussion below). Michelson and Pease finally obtained the first measurement of a stellar diameter during the winter of 1920, that of Betelgeuse ( $\alpha$ Orionis), a red supergiant. They found an angular diameter of 0.047", that is a linear diameter 400 times larger than that of the Sun, given the distance of Betelgeuse (650 light years). Five more bright stars were also resolved. Anderson used the same observing technique with the $2.5 \mathrm{~m}$ telescope at Mount Wilson to resolve very tight spectroscopic binaries (cf. Capella). Michelson and Pease did not stop there: they undertook the construction of a $15 \mathrm{~m}$ optical beam based on the same principle, and began to use it in 1929. Unfortunately, the mechanical vibrations and deformations were such that this instru- 
ment was too delicate. It was abandoned in 1930, without having reached its limiting angular resolution of 0.01 ". It was not until 1956 that optical stellar interferometry was reborn and again, according to a principle different from that of Fizeau. Fizeau-type interferometry had indeed acquired a reputation of great operational difficulty. The intensity interferometry by the two radar manufacturers Hanbury Brown and Twiss (Australia) was then set up, based on an entirely new approach: the measurement of the space correlation of the stellar intensity fluctuations. Their interferometer made it possible to measure the diameter of 32 blue stars with a very high precision $(<0.0005$ ") and to detect a few very tight binaries. But it is in the field of radio astronomy that the development of interferometry with independent telescopes became the most spectacular in the 1950s. At optical wavelengths, we had to wait until 1975 when Prof. Antoine Labeyrie and his close collaborators succeeded in combining the light from two independent telescopes. A boost then took place in the successful development of optical/IR interferometry.

\section{Light coherence}

When we previously established the relationship between the angular diameter $\Delta$ of a source and the separation $B$ between the two apertures of the interferometer for which the interference fringes disappear, we made two approximations that do not really apply to usual conditions of observation. We first assumed that the waves falling on Young's screen were planes, that is to say coming from a very distant point-like source and also that they were purely monochromatic. In addition, the holes through which light is being scattered should have finite dimensions. Therefore, we shall later take into account the finite dimensions of the apertures (see Section 6) but let us first consider the effects due to the finite dimension of the source, also considering a spectral range having a certain width and to do so, we shall make use of some elements of the theory of light coherence. This theory consists essentially in a statistical description of the properties of the radiation field in terms of the correlation between electromagnetic vibrations at different points in the field.

\subsection{Quasi-monochromatic light}

The light emitted by a real source (see Fig. 55) is of course not monochromatic. As in the case of a monochromatic wave, the intensity of such a radiation field at any point in space is defined by

$$
I=\left\langle V(t) V(t)^{*}\right\rangle .
$$

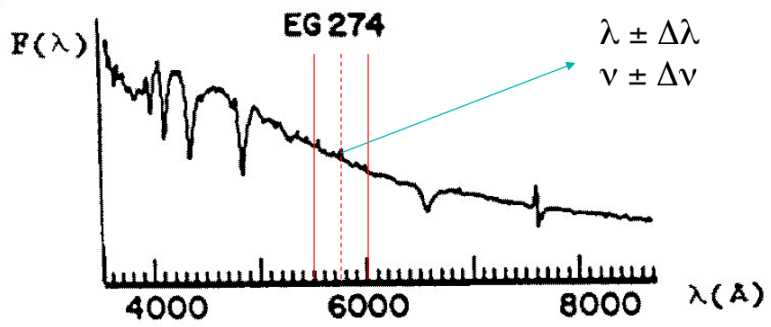

Figure 55. Stars do not emit monochromatic light. Quasi monochromatic light is assumed to be emitted at the wavelength $\lambda$ (resp. the frequency $v$ ) within the bandwidth $\pm \Delta \lambda$ (resp. $\pm \Delta v$ ).

In order to determine the electric field created by such a source, emitting within a certain frequency range $\pm \Delta v$, we must sum up the fields due to all the individual monochromatic 
components such that the resulting electric field $V(z, t)$ is given by the real part of the following expression:

$$
V(z, t)=\int_{v-\Delta v}^{v+\Delta v} a\left(v^{\prime}\right) \exp \left[i 2 \pi\left(v^{\prime} t-z / \lambda^{\prime}\right)\right] d v^{\prime} .
$$

While a monochromatic beam of radiation corresponds to an infinitely long wave train, it can easily be shown that the superposition of multiple infinitely long wave trains, with nearly similar frequencies, results in the formation of wave groups. Indeed, the expression of the electric field established in Eq. (68) can be reduced as follows. Insert in the integral of Eq. (68) the following factors: $\exp (i 2 \pi(v t-z / \lambda)) \exp (-i 2 \pi(v t-z / \lambda))$. We then find that Eq. (68) may be rewritten as

$$
V(z, t)=A(z, t) \exp [i 2 \pi(v t-z / \lambda)]
$$

where

$$
A(z, t)=\int_{v-\Delta v}^{v+\Delta v} a\left(v^{\prime}\right) \exp \left\{i 2 \pi\left[\left(v^{\prime}-v\right) t-z\left(1 / \lambda^{\prime}-1 / \lambda\right)\right]\right\} d v^{\prime} .
$$

Expression (69) represents that of a monochromatic wave of frequency $v$ whose amplitude $A(z, t)$ varies periodically with a much smaller frequency $\Delta v$ (cf. beat phenomenon). As an exercise, it is instructive to set $a\left(v^{\prime}\right)$ constant in Eq. (70) and establish that indeed $A(z, t)$ varies as a function of time with a frequency $\Delta v$. This modulation therefore effectively splits the monochromatic wave trains having different but nearly similar frequencies into wave groups whose length is of the order of $\lambda^{2} / \Delta \lambda$, with $\Delta \lambda=-c \Delta v / v^{2}$ and the frequency of the order $\Delta v$ (see Figure 56).

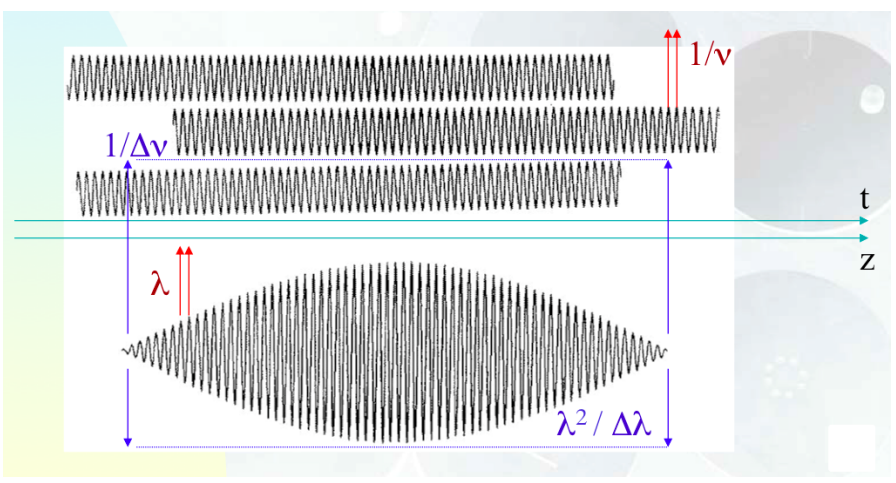

Figure 56. Superposition of long wave trains having quite similar frequencies $v^{\prime}$ in the range $v \pm \Delta v$ (resp. wavelengths $\lambda^{\prime}$ in the range $\lambda \pm \Delta \lambda$ ) results in the propagation of long wave trains with the frequency $v$ (resp. wavelength $\lambda$ ) but which amplitude $A(z, t)$ is varying with a lower frequency $\Delta v$ (resp. longer wavelength $\lambda^{2} / \Delta \lambda$ ).

\subsection{Visibility of the interference fringes}

What becomes the visibility of the interference fringes in the Young's hole experiment for the case of a quasi-monochromatic source having a finite dimension?

We can re-write the expression of the intensity $I_{q}$ at point $q$ as indicated below (see Eqs. (71)-(74)). It is assumed that the holes placed at the points $P_{1}, P_{2}$ in the Young plane have the same aperture size (i.e. $V_{1}(t)=V_{2}(t)$ ) and that the propagation times of the light between $P_{1}$ (resp. $P_{2}$ ) and $q$ are $t_{1 q}$ (resp. $t_{2 q}$, see Fig. 57) :

$$
I_{q}=\left\langle V_{q}^{*}(t) V_{q}(t)\right\rangle
$$




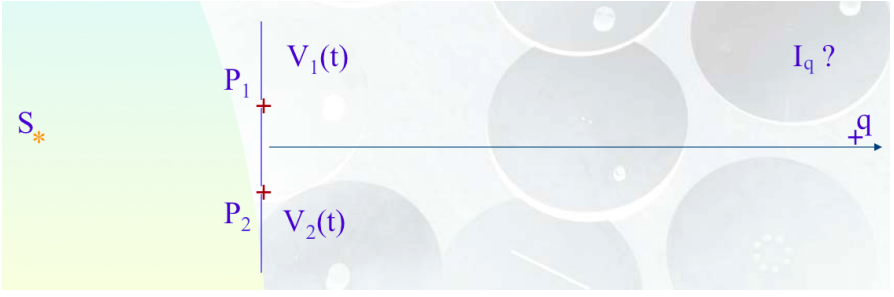

$$
V_{q}(t)=V_{1}\left(t-t_{1 q}\right)+V_{2}\left(t-t_{2 q}\right)
$$

and after a mere change of the time origin

$$
V_{q}(t)=V_{1}(t)+V_{2}(t-\tau)
$$

where we have defined

$$
\tau=t_{2 q}-t_{1 q} .
$$

It follows that Eq. (71) can be easily transformed into (75) where (76) represents the complex degree of mutual coherence, and the intensity $I=\left\langle V_{1} V_{1}^{*}\right\rangle=\left\langle V_{2} V_{2}^{*}\right\rangle$. Equation (75) is used to find what is the intensity distribution of the interference fringes in the observation plane. The complex degree of mutual coherence $\gamma_{12}(\tau)$ (see Eq. (76)) is a fundamental quantity whose significance will be highlighted when calculating the visibility of the interference fringes. By means of (69), this function $\gamma_{12}(\tau)$ can still be expressed as (77), and if $\tau<<1 / \Delta v$ (i.e. the difference between the arrival times of the two light rays is less than the beat period $1 / \Delta v$ of the quasi-monochromatic radiation), we can give it the form (78):

$$
\begin{gathered}
I_{q}=I+I+2 I \operatorname{Re}\left[\gamma_{12}(\tau)\right], \\
\gamma_{12}(\tau)=\left\langle V_{1}^{*}(t) V_{2}(t-\tau)\right\rangle / I, \\
\gamma_{12}(\tau)=\left\langle A_{1}^{*}(z, t) A_{2}(z, t-\tau)\right\rangle \exp (-i 2 \pi v \tau) / I,
\end{gathered}
$$

and if $\tau<<1 / \Delta v$

$$
\gamma_{12}(\tau)=\left|\gamma_{12}(\tau=0)\right| \exp \left(i \beta_{12}-i 2 \pi \nu \tau\right) .
$$

Equation (75) can then be rewritten as (79) and in this case the visibility $v$ of the interference fringes is $\left|\gamma_{12}(\tau=0)\right|$ (see Eq. (80)), $I_{\max }$ and $I_{\min }$ representing the brightest and weakest fringe intensities.

$$
I_{q}=I+I+2 I\left|\gamma_{12}(\tau=0)\right| \cos \left(\beta_{12}-2 \pi v \tau\right)
$$

and

$$
v=\left(\frac{I_{\max }-I_{\min }}{I_{\max }+I_{\min }}\right)=\left|\gamma_{12}(\tau=0)\right| .
$$

We will see in the next section that the module of $\gamma_{12}(\tau=0)$ is directly related to the structure of the source that we are observing. 
We propose hereafter to the reader to answer the two following questions. What is the value of $\left|\gamma_{12}(\tau=0)\right|$ in the Young's holes experiment for the case of a monochromatic wave, two point-like holes and an infinitely small point-like source? And what can we say about the source when $\left|\gamma_{12}(\tau=0)\right|=0$ ?

Let us now evaluate what $\gamma_{12}(\tau=0)$ is for the case we are interested in, namely an extended source emitting quasi-monochromatic light. This leads us directly to study the notion of the spatial coherence of light.

\subsection{Spatial coherence}

Let us thus evaluate Eq. (76) for the case $\tau=0$. We find

$$
\gamma_{12}(\tau=0)=\left\langle V_{1}^{*}(t) V_{2}(t)\right\rangle / I .
$$

If $V_{i 1}(t)$ and $V_{i 2}(t)$ represent the electric fields at $P_{1}$ and $P_{2}$ due to a small surface element $d S_{i}$ on the source $S$ (see Fig. 58), we find that the fields $V_{1}(t)$ and $V_{2}(t)$ can be expressed as

$$
\begin{aligned}
& V_{1}(t)=\sum_{i=1}^{N} V_{i 1}(t), \\
& V_{2}(t)=\sum_{i=1}^{N} V_{i 2}(t) .
\end{aligned}
$$

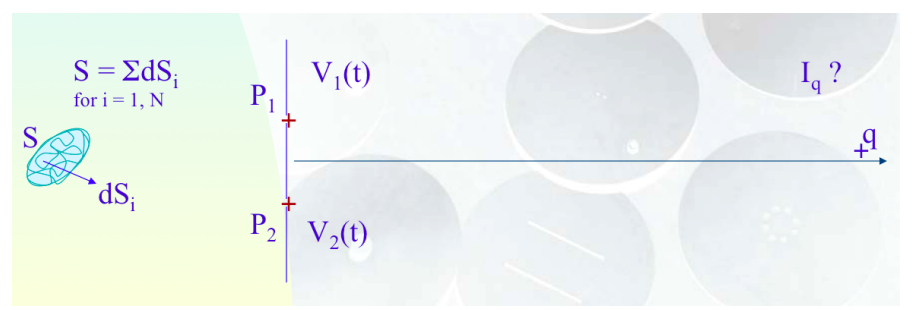

Figure 58. The extended source $S$ is assumed to be composed of a large number of infinitesimal surface elements $d S_{i}$.

It is assumed that the distinct points $i$ of the source are separated by small distances compared to the wavelength $\lambda$ of the light they emit in a mutually incoherent manner. Obtaining the expression (83) for $\gamma_{12}(0)$ is then immediate

$$
\gamma_{12}(0)=\left[\sum_{i=1}^{N}\left\langle V_{i 1}^{*} V_{i 2}\right\rangle+\sum_{i \neq j}^{N}\left\langle V_{i 1}^{*} V_{j 2}\right\rangle\right] / I .
$$

For an incoherent light source, the second summation appearing in (83) is obviously equal to zero. As a reminder, the contributions $V_{i j}(t)$ can be expressed as

$$
\begin{gathered}
V_{i 1}(t)=\frac{a_{i}\left(t-r_{i 1} / c\right)}{r_{i 1}} \exp \left[i 2 \pi v\left(t-r_{i 1} / c\right)\right], \\
V_{i 2}(t)=\frac{a_{i}\left(t-r_{i 2} / c\right)}{r_{i 2}} \exp \left[i 2 \pi v\left(t-r_{i 2} / c\right)\right]
\end{gathered}
$$

where $r_{i 1}$ and $r_{i 2}$ respectively represent the distances between the element $i$ of the source and the points $P_{1}$ and $P_{2}$. The products $V_{i 1}^{*}(t) V_{i 2}(t)$ simplify themselves as 


$$
\left.V_{i 1}^{*}(t) V_{i 2}(t)=\frac{\left|a_{i}\left(t-r_{i 1} / c\right)\right|^{2}}{r_{i 1} r_{i 2}} \exp \left[-i 2 \pi v\left(r_{i 2}-r_{i 1}\right) / c\right)\right],
$$

as long as the following condition is verified

$$
\left|r_{i 1}-r_{i 2}\right| \leq c / \Delta v=\lambda^{2} / \Delta \lambda=\ell .
$$

We thus see how to naturally introduce the coherence length $\ell$ which characterizes the precision with which we must obtain the equality between the optical paths in order to be able to observe interference fringes (typically 2.5 microns in the visible for $\Delta \lambda=1000 \AA$ ).

\subsection{Zernicke-van Cittert theorem}

To obtain the mutual intensity due to the whole source, it suffices to insert in the expression (83), the relation (85) using (87). The result is Eq. (88), also known as the Zernicke-van Cittert Theorem

$$
\begin{gathered}
I(s) d s=\left|a_{i}(t-r / c)\right|^{2}, \\
\left.\gamma_{12}(0)=\int_{S} \frac{I(s)}{r_{1} r_{2}} \exp \left[-i 2 \pi\left(r_{2}-r_{1}\right) / \lambda\right)\right] d s / I .
\end{gathered}
$$

When the distance between the source and the screen is very large, the expression of this theorem can be simplified as follows. Let us adopt the orthonormal coordinate system $(x, y, z)$ shown in Fig. 59 such that the coordinates of the two elements $P_{1}$ and $P_{2}$ of the interferometer are respectively $(X, Y, 0)$ and $(0,0,0)$ and those of an infinitesimal element $d S_{i}$ of the source $\left(X^{\prime}, Y^{\prime}, Z^{\prime}\right)$. It is then easy to find, by means of a relation analogous to (61), that

$$
\left|r_{2}-r_{1}\right|=\left|P_{2} P_{i}-P_{1} P_{i}\right|=\left|-\frac{\left(X^{2}+Y^{2}\right)}{2 Z^{\prime}}+(X \zeta+Y \eta)\right|
$$

where

$$
\zeta=\frac{X^{\prime}}{Z^{\prime}}, \eta=\frac{Y^{\prime}}{Z^{\prime}}
$$

represent the angular coordinates of the source measured from the interferometer. Using the two last relations, one can easily transform the expression (88) into (91). The $X, Y$ coordinates in the first member of $\gamma_{12}(0, X / \lambda, Y / \lambda)$ represent the position of one element of the interferometer relative to the other. One often defines $u=X / \lambda$ and $v=Y / \lambda$ which are quantities having the dimensions of the inverse of an angle, thus of angular space frequencies.

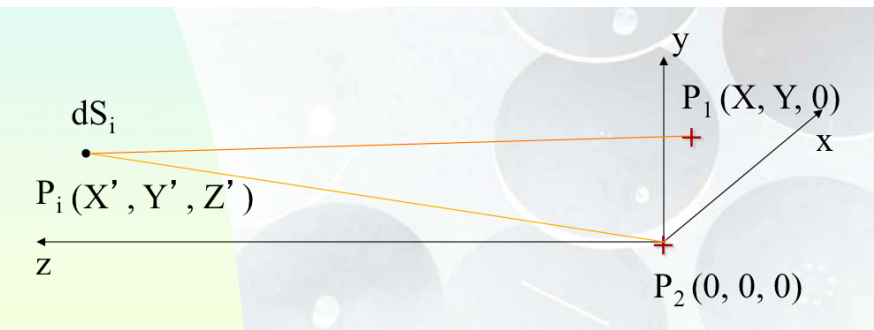

Figure 59. Positions of the two elements $P_{1}$ and $P_{2}$ of the interferometer and of the infinitesimal element $P_{i}$ of the source assuming that the distance $Z^{\prime}>>\left|X^{\prime}\right|,\left|Y^{\prime}\right|,|X|$ or $|Y|$.

Apart from a multiplicative factor, we thus find that the visibility of the fringes (the function $\left.\left|\gamma_{12}(\tau=0)\right|\right)$ is simply the modulus of the Fourier transform of the normalized surface brightness $I^{\prime}$ of the source (Eq. (92)). 


$$
\gamma_{12}(0, X / \lambda, Y / \lambda)=\exp \left(-i \phi_{X, Y}\right) \iint_{S} I^{\prime}(\zeta, \eta) \exp [-i 2 \pi(X \zeta+Y \eta) / \lambda] d \zeta d \eta
$$

with

$$
I^{\prime}(\zeta, \eta)=\frac{I(\zeta, \eta)}{\iint_{S} I\left(\zeta^{\prime}, \eta^{\prime}\right) d \zeta^{\prime} d \eta^{\prime}} .
$$

In terms of the angular space frequencies $u=X / \lambda, v=Y / \lambda$, Eq. (91) becomes

$$
\gamma_{12}(0, u, v)=\exp \left(-i \phi_{u, v}\right) \iint_{S} I^{\prime}(\zeta, \eta) \exp [-i 2 \pi(u \zeta+v \eta)] d \zeta d \eta
$$

By a simple inverse Fourier transform, it is then possible to recover the (normalized) surface brightness of the source with an angular resolution equivalent to that of a telescope whose effective diameter would be equal to the baseline of the interferometer consisting of two independent telescopes

$$
I^{\prime}(\zeta, \eta)=\iint \gamma_{12}(0, u, v) \exp \left(i \phi_{u, v}\right) \exp [i 2 \pi(\zeta u+\eta v)] d u d v .
$$

Equations (93) and (94) thus clearly highlight the power of the complex degree of mutual coherence since they make it possible to link the visibility and the normalized intensity distribution of the source by means of the Fourier transform $v=\left|\gamma_{12}(0)\right|=\left|F T\left[I^{\prime}\right]\right|$, and its inverse. Aperture synthesis consists in observing a maximum number of visibilities of the source, thus trying to cover as well as possible the $(u, v)$ plane from which we shall try, sometimes with some additional assumptions, to determine the structure of the source from the inverse Fourier transform (94) in which the integrant is not the visibility (i.e. the module of the complex degree of mutual coherence) but the complex degree of mutual coherence itself, within the factor $\exp \left(i \phi_{x, y}\right)$. It is now good to remind some specific properties of the Fourier transform.

\subsection{Some remarkable properties of the Fourier transform and applications}

Let us remind that the Fourier transform of the function $f(x)$, denoted $F T[f(x)](s)$, where $x \in \mathbb{R}$, is the function

$$
F T[f(x)](s)=\int_{-\infty}^{\infty} f(x) \exp (-2 i \pi s x) d x
$$

where $s \in \mathfrak{R}$. The functions $f$ and $F T[f]$ form a Fourier pair. The function $F T[f]$ exists if the function $f(x)$ is bounded, summable and has a finite number of extrema and discontinuities. This does not necessarily imply that the inverse Fourier transform, denoted $F T^{-1}[F T[f]]$ transform is $f$. For the Fourier transformation to be reciprocal,

$$
f(x)=\int_{-\infty}^{\infty} F T[f](s) \exp (2 i \pi x s) d s,
$$

it suffices $f$ to be of summable square, i.e. that the following integral exists

$$
\int_{-\infty}^{\infty}|f(x)|^{2} d x
$$


The definition of $F T$ can be extended to the distributions. The $F T$ of a distribution is not necessarily of summable square. Let us also note that the functions $f$ and $F T[f]$ can be real or complex.

We can generalize the $F T$ to several dimensions, by defining $f$ on the space $\mathfrak{R}^{n}$. Let $\mathbf{r}$, $\mathbf{w} \in \mathfrak{R}^{\mathbf{n}}$, we then have

$$
F T[f](\mathbf{w})=\int_{-\infty}^{\infty} f(\mathbf{r}) \exp (-2 i \pi \mathbf{w r}) d \mathbf{r} .
$$

As a reminder, if $f(t)$ designates a function of time, $F T[f](s)$ represents its content as a function of time frequencies. Similarly, if $f(\mathbf{r})$ is defined on $\mathfrak{R}^{2}$, where $\mathfrak{R}^{2}$ represents a twodimensional space, the function $F T[f](\mathbf{w})$ represents the space frequency content of $f(\mathbf{r})$, where $\mathbf{w} \in \mathfrak{R}^{2}$.

Among the interesting properties of the Fourier transform, let us remind:

\subsubsection{Linearity:}

$$
F T[a f]=a F T[f],
$$

with the constant $a \in \mathfrak{R}$,

$$
F T[f+g]=F T[f]+F T[g] .
$$

\subsubsection{Symmetry and parity:}

The considerations of symmetry are very useful during the study of the Fourier transform. Let $P(x)$ and $I(x)$ be the even and odd parts of $f(x)$ such that

$$
f(x)=P(x)+I(x)
$$

we find that

$$
F T[f](s)=2 \int_{0}^{\infty} P(x) \cos (2 \pi x s) d x-2 i \int_{0}^{\infty} I(x) \sin (2 \pi x s) d x .
$$

From this result, we can deduce for instance that if $f(x)$ is real, the real part of $F T[f](s)$ will be even and its imaginary part will be odd whereas if $f(x)$ is complex, the imaginary part of $F T[f](s)$ will be even and its real part will be odd.

\subsubsection{Similarity:}

The relationship of similarity is the following one

$$
F T[f(x / a)](s)=|a| F T[f(x)](a s)
$$

where $a \in \mathfrak{R}$, is a constant. The dilation of a function causes a contraction of its Fourier transform. This very visual property is very useful to understand that a function whose support is very compact, has a very spread transform. In the analysis of temporal frequencies, one would state that a pulse of very short duration results in a very broad frequency spectrum, that is to say, contains frequencies all the higher as the pulse is brief. This is the classical relation of the spectrum of a wave packet, according to which the knowledge of the properties of a signal cannot be arbitrarily precise both in time and in frequency. 


\subsubsection{Translation:}

The translation relation is written as

$$
F T[f(x-a)](s)=\exp (-2 i \pi a s) F T[f(x)](s) .
$$

A translation of the function in its original space corresponds to a phase rotation of its Fourier transform in the transformed space.

\subsubsection{Door function:}

The door function, denoted $\Pi(x)$, is defined by (see Fig. 60)

$$
\Pi(x)=1 \quad \text { if } x \in[-0.5,0.5], \quad \text { and } \quad \Pi(x)=0 \quad \text { otherwise. }
$$

It is easy to find that

$$
F T[\Pi(x)](s)=\operatorname{sinc}(s)=\frac{\sin (\pi s)}{\pi s} .
$$

Applying the similarity relation, we also find that

$$
F T[\Pi(x / a)](s)=|a| \operatorname{sinc}(a s)=|a| \frac{\sin (\pi a s)}{\pi a s} .
$$

The door function is also sometimes called the window function or simply window.

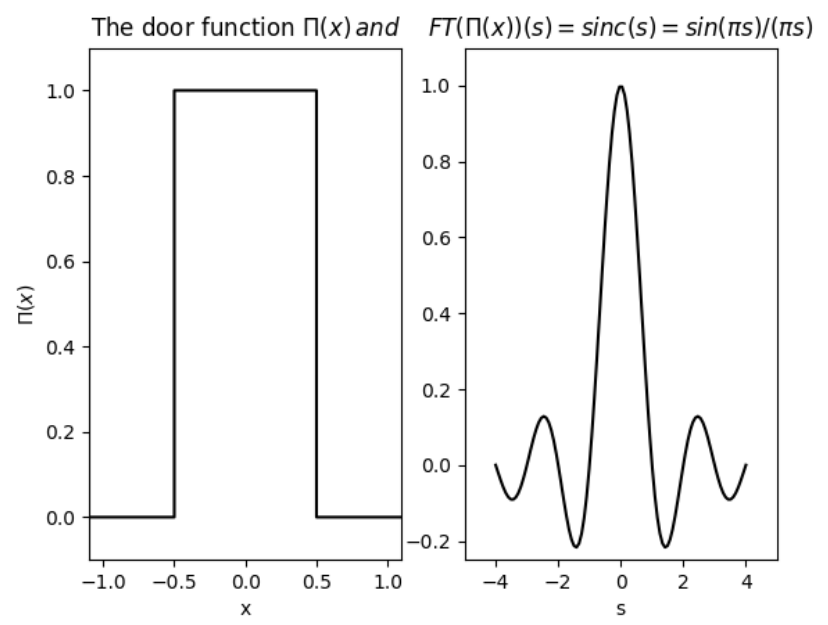

Figure 60. The door function and its Fourier transform (cardinal sine).

\subsubsection{Distribution of Dirac:}

The Dirac distribution, also called Dirac peak, is noted $\delta(x)$. It is defined by the following integral, which exists only in the sense of the distributions

$$
\delta(x)=\int_{-\infty}^{\infty} \exp (2 i \pi x s) d s .
$$

Its Fourier transform is therefore 1 in the interval $]-\infty,+\infty[$ since $\delta(x)$ appears above as the inverse Fourier transform of 1. 


\subsubsection{Applications:}

We propose hereafter several astrophysical applications that make use of the previous remarkable properties of the Fourier transform.

Let us first consider the case of a double star which two point-like components are equally bright and separated by an angle $2 \zeta_{0}$. Making use of Eqs. (80), (92) and (93), one may easily establish using the properties (104) and (108) that the normalized intensity $I^{\prime}(\zeta)$ takes the simple form

$$
I^{\prime}(\zeta)=\frac{\delta\left(\zeta-\zeta_{0}\right)+\delta\left(\zeta+\zeta_{0}\right)}{2}
$$

and that the visibility $v$ measured with an interferometer composed of 2 telescopes separated by the baseline $X$ is given by the expression

$$
v=\left|\gamma_{12}(0)\right|=\left|\cos \left(2 \pi \zeta_{0} u\right)\right|
$$

where $u=X / \lambda$.

A second nice application consists in deriving the visibility of the interference fringes measured with the same interferometer of a $1-D$ Gaussian star which intensity $I(\zeta)$ distribution is given by the following expression

$$
I(\zeta)=\exp \left(\frac{-4 \ln (2) \zeta^{2}}{\mathrm{FWHM}^{2}}\right)
$$

where $F W H M$ represents the angular full width at half maximum of the $1-D$ Gaussian star. The expression of the corresponding visibility is then easily found to be

$$
v=\left|\gamma_{12}(0, u)\right|=\exp \left(\frac{-\pi^{2} u^{2} \mathrm{FWHM}^{2}}{4 \ln (2)}\right),
$$

and we notice that narrower is the angular size of the star, broader is its visibility content in angular space frequencies.

In the third proposed application, we ask to establish the expression of the visibility of a $2-D$ uniformly bright square star which each angular side is $\zeta_{0}$, i.e. $I(\zeta)=$ Cte $\Pi\left(\zeta / \zeta_{0}\right) \Pi\left(\eta / \zeta_{0}\right)$.

The expression to be derived is the following one

$$
v=\left|\gamma_{12}(0, u, v)\right|=\left|\frac{\sin \left(\pi \zeta_{0} u\right)}{\pi \zeta_{0} u} \frac{\sin \left(\pi \zeta_{0} v\right)}{\pi \zeta_{0} v}\right| .
$$

Finally, a generalization of the previous application consists in deriving the visibility of a star which is seen as a projected $2-D$ uniform circular disk which angular radius is $\rho_{U D}$ and its angular diameter $\theta_{U D}$.

Due to the circular symmetry of the problem, it is convenient to make use of polar coordinates in Eq. (91) as follows:

$$
\begin{aligned}
u & =X / \lambda=R \cos (\psi) / \lambda \\
v & =Y / \lambda=R \sin (\psi) / \lambda,
\end{aligned}
$$

where $R$ denotes the baseline between the two telescopes of the interferometer, and

$$
\begin{aligned}
\zeta & =\theta \cos (\phi) \\
\eta & =\theta \sin (\phi) .
\end{aligned}
$$


Eq. (91) then transforms into

$$
\left|\gamma_{12}(0, R / \lambda, \psi)\right|=\left|\frac{1}{\pi \rho_{U D}^{2}} \int_{0}^{\rho_{U D}} \theta \int_{0}^{2 \pi} \exp [-i 2 \pi \theta R / \lambda(\cos (\phi) \cos (\psi)+\sin (\phi) \sin (\psi))] d \phi d \theta\right| .
$$

Making use of the additional changes of variables

$$
\begin{array}{r}
z=2 \pi \theta R / \lambda \\
\Phi=\pi / 2-\phi+\psi,
\end{array}
$$

Eq. (116) becomes

$$
\left|\gamma_{12}(0, R / \lambda, \psi)\right|=\left|\left(\frac{\lambda}{2 \pi R}\right)^{2} \frac{1}{\pi \rho_{U D}^{2}} \int_{0}^{2 \pi \rho_{U D} R / \lambda} \theta \int_{-3 \pi / 2+\psi}^{\pi / 2+\psi} \cos (z \sin (\Phi)) d \Phi d \theta\right| .
$$

Reminding the definition of the zero order Bessel function $J_{0}(x)$

$$
J_{0}(x)=\frac{1}{\pi} \int_{0}^{\pi} \cos [x \sin (\theta)] d \theta,
$$

and the relation existing between $J_{0}(x)$ and the first order Bessel function $J_{1}(x)$, namely

$$
x J_{1}(x)=\int x^{\prime} J_{0}\left(x^{\prime}\right) d x^{\prime}
$$

Eq. (118) successively reduces to

$$
\left|\gamma_{12}(0, R / \lambda)\right|=\left|\left(\frac{\lambda}{2 \pi R}\right)^{2} \frac{1}{\pi \rho_{U D}^{2}} 2 \pi \int_{0}^{2 \pi \rho_{U D} R / \lambda} z J_{0}(z) d z\right|
$$

and

$$
\left|\gamma_{12}(0, R / \lambda)\right|=\left|2 \frac{J_{1}\left(2 \pi \rho_{U D} R / \lambda\right)}{2 \pi \rho_{U D} R / \lambda}\right| .
$$

We thus find that the expression (80) of the fringe visibility for the case of a star seen as a projected 2-D uniform circular disk with an angular dimater $\theta_{U D}=2 \rho_{U D}$ is

$$
v=\left(\frac{I_{\max }-I_{\min }}{I_{\max }+I_{\min }}\right)=\left|\gamma_{12}(0, u)\right|=\left|\frac{2 J_{1}\left(\pi \theta_{U D} u\right)}{\pi \theta_{U D} u}\right|,
$$

where we have set $u=R / \lambda$. As a reminder, the Bessel function has the following properties

$$
\begin{array}{r}
J_{1}(x=3.8317 \ldots)=0 \\
\lim _{x \rightarrow 0} \frac{J_{1}(x)}{x}=1 / 2,
\end{array}
$$

which allow us to easily understand the behavior of the visibility function illustrated in Fig. 61.

One could then wonder whether it is possible to observe interferometric fringes from our nearest star, i.e. the Sun? Figure 62 illustrates such fringes in while light obtained on 9th 


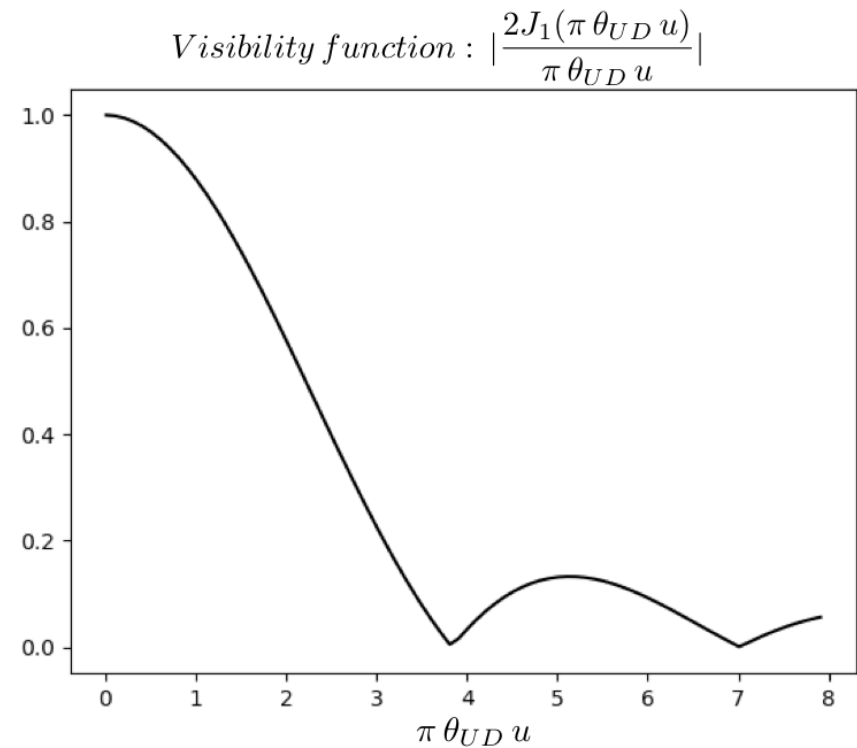

Figure 61. Visibility function expected for a star consisting of a uniformly bright circular disk with an angular diameter $\theta_{U D}$.

of April 2010 using a micro interferometer consisting of 2 holes with a diameter of 11.8 $\mu$ separated by a baseline of $29.4 \mu$. This micro-interferometer was placed in front of the objective of an EOS 5D Canon camera. Since the picture was taken in white light, it is possible to see the effects due to color dispersion. It is then easy to get an estimate of the fringe visibility, using Eq. (123), assuming that the Sun is a uniform disk with an angular diameter of 30 '.
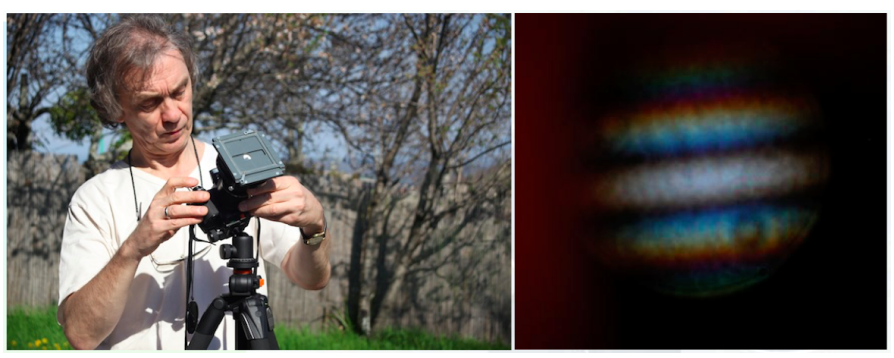

Figure 62. Solar fringes photographed with an EOS $5 \mathrm{D}$ Canon camera in front of which was set a micro-interferometer consisting of two holes having a diameter of $11.8 \mu$ separated by a baseline of $29.4 \mu$.

\section{Some examples of interferometers}

One of the most respected sanctuaries of optical interferometry is located on the plateau of Caussols, north of Grasse, in the south of France. The I2T (in French, "Interféromètre à 2 Télescopes"), made of 2 telescopes with an aperture of $26 \mathrm{~cm}$ each and separated by a baseline of up to $144 \mathrm{~m}$ was characterized by an angular resolution $\Phi \sim 0.001^{\prime \prime}$ attainable for objects with an apparent magnitude brighter than $V_{\text {lim }} \sim 6$ (see Figure 63, left). First interference fringes were obtained on Vega in 1975 (Fig. 63, right). About twenty angular diameters of stars have been measured using the same I2T by Prof. Antoine Labeyrie and his close collaborators. In order to equalize the light paths collected from the stars passing through the two telescopes, optical delay lines are mandatory. These have been successfully used for the first time in 1975 (see Fig. 64 for an illustration of how delay lines work and Figs. 65 and 66 for views on modern optical delay lines in use at the VLTI, ESO, Chile). 


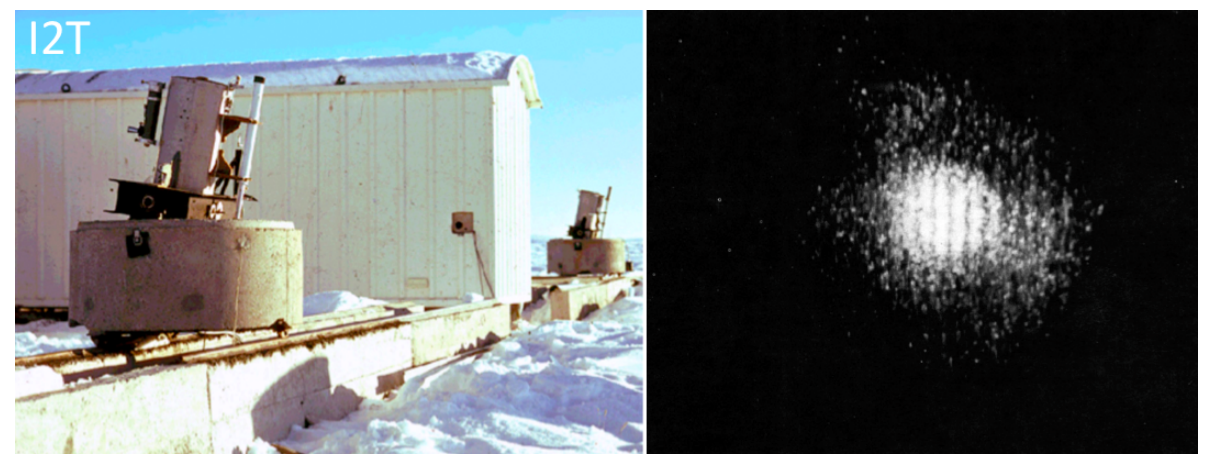

Figure 63. First fringes obtained with the I2T on Vega (Labeyrie et al. 1975, (c) Observatoire de la Côte d'Azur ).

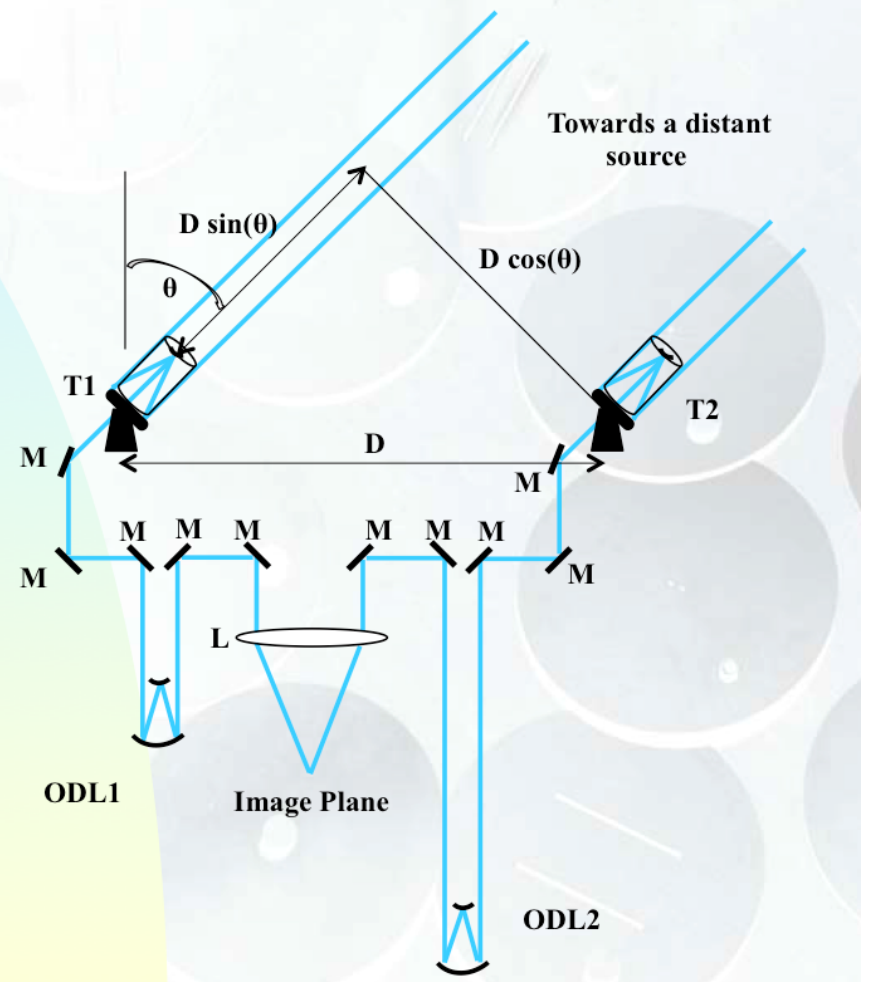

Figure 64. Use of optical delay lines to compensate for the continuous change in the lengths of the two light paths as the Earth rotates.

The GI2T (in French, "Grand Interféromètre à 2 Télescopes") composed of two $1.5 \mathrm{~m}$ telescopes was subsequently used by the same team. The two big telescopes could in principle be set $2 \mathrm{~km}$ apart, corresponding to an angular resolution $\Phi \sim 0.0001^{\prime \prime}$ (see Fig. 67).

Since the beginning of the $21^{\text {st }}$ century, the modern sanctuary of stellar interferometry and aperture synthesis is undoubtedly the Very Large Telescope Interferometer (VLTI) of ESO (Southern European Observatory), located in Chile on Mount Paranal (see Fig. 68). The VLTI is a European interferometer that can re-combine the signal from 2, 3 or 4 telescopes 


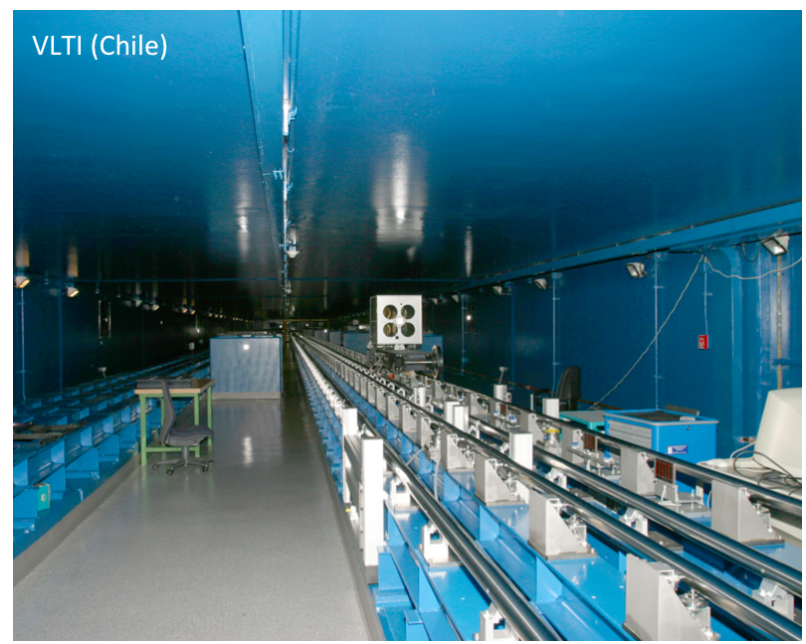

Figure 65. View inside the optical delay line tunnel of the VLTI at ESO, Paranal, Chile. (C) ESO.

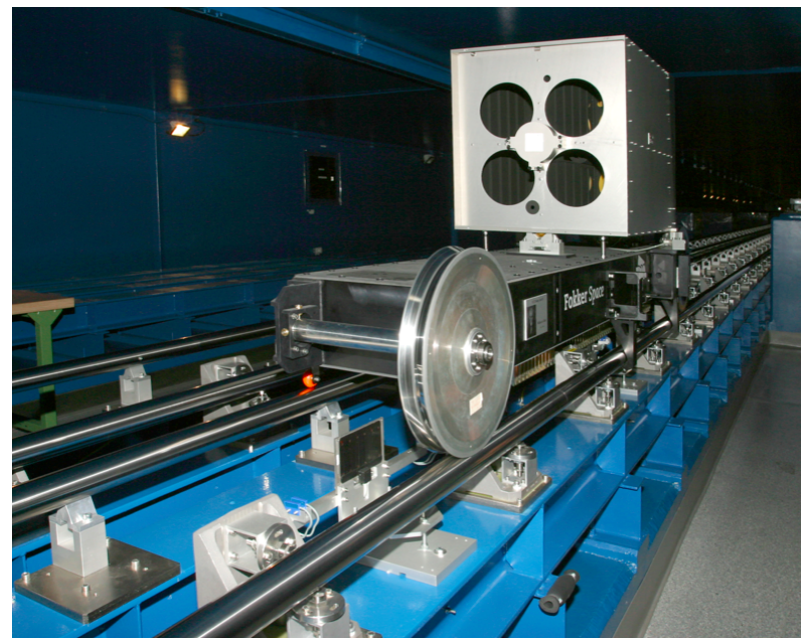

Figure 66. Zoom on one of the optical delay lines used in the tunnel of the VLTI at ESO, Paranal, Chile. (c) ESO.

depending on the instrument used. It has 4 telescopes of $8.2 \mathrm{~m}$ and 4 mobile telescopes of $1.8 \mathrm{~m}$. Only telescopes of the same size can be re-combined together. The auxiliary telescopes of $1.8 \mathrm{~m}$ can be easily moved allowing a better coverage of the $u, v$ plane. The maximum base length of this interferometer is about $200 \mathrm{~m}$.

CHARA is another very performing interferometer located on the heights of Los Angeles, California (see Fig. 69). It is installed on the historic observatory of Mount Wilson. Remember that it was with the $2.5 \mathrm{~m}$ telescope of this observatory that the first measurement of a stellar diameter was made by Michelson and Pease by installing a beam of $7 \mathrm{~m}$ at the top of the telescope. The CHARA interferometric array, operational since 1999 is composed of 6 telescopes of $1 \mathrm{~m}$ in diameter. These 6 telescopes can be either re-combined by 2 , by 3 since 2008 and recently the 6 together. The maximum base length of this interferometer is $330 \mathrm{~m}$ allowing to achieve an angular resolution of $200 \mu$ arcsec.

It is mainly used for angular diameter measurements but also for the detection and characterization of tight binary stars as well as for the detection of exo-zodiacal clouds (clouds of dust gravitating around the stars). Another famous optical/IR interferometer is the Keck 


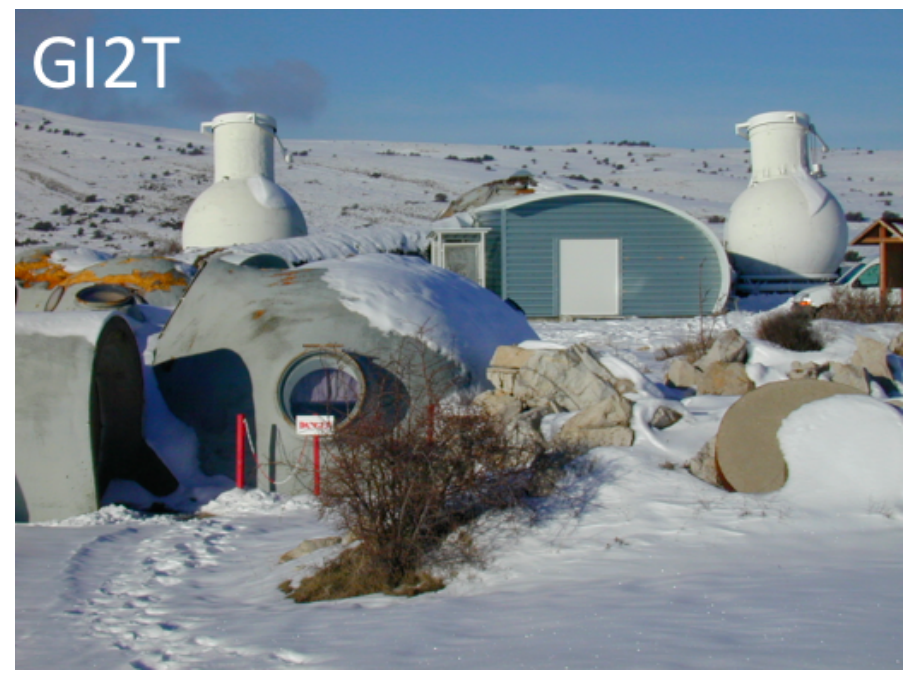

) .

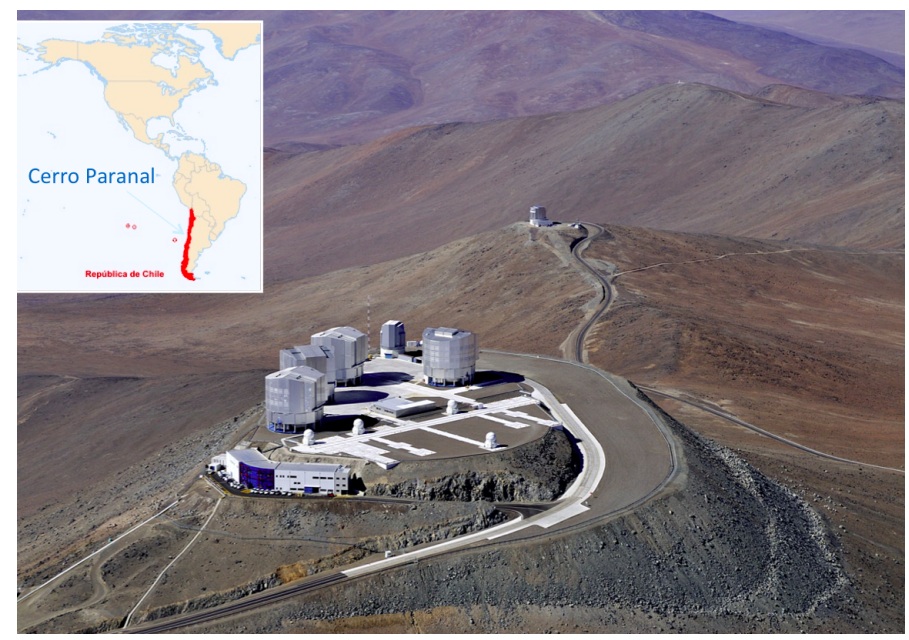

Figure 67. The GI2T constructed by Antoine Labeyrie and his close collaborators on the plateau of Caussols, north of Grasse, near Nice (France, (c) Observatoire de la Côte d'Azur

Figure 68. The Very Large Telescope Interferometer (VLTI) at the top of Paranal (Chile, (c) ESO).

Interferometer made of two 10m telescopes separated by a fixed baseline of $85 \mathrm{~m}$ (see Fig. 70) on top of Mauna Kea (Hawaii, USA).

\section{Three important theorems and some applications}

When we previously established the relation existing between the structure of a celestial source and the visibility of the fringes observed with an interferometer (Sections 3.3 and 4.4), we implicitly assumed that the size of the apertures was infinitely small (pinhole apertures). Use of the fundamental theorem allows one to calculate the response function of an interferometer equipped with finite size apertures. This theorem actually formalizes, in mathematical terms, the physical connection existing between the focal plane and the pupil plane of an optical instrument (telescope, interferometer, grating, etc.). Use of the convolution theorem will then enable us to establish the relation between a celestial source that is extended and its observed image in the focal plane of an optical instrument. Finally, the 


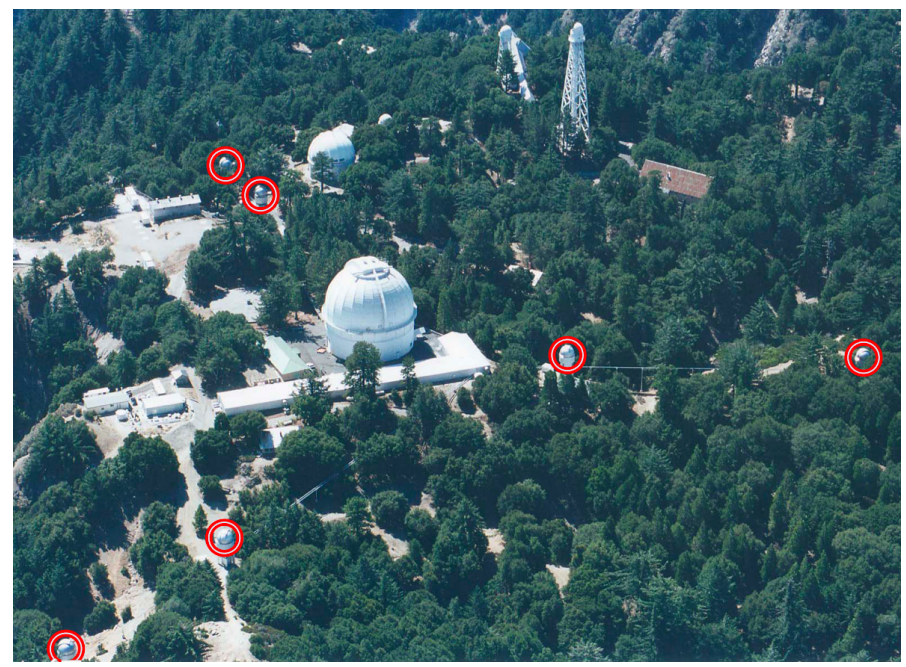

Figure 69. The CHARA

interferometer composed of six $1 \mathrm{~m}$ telescopes at Mount Wilson Observatory (California, USA). (c) The Observatories of the Carnegie Institution.

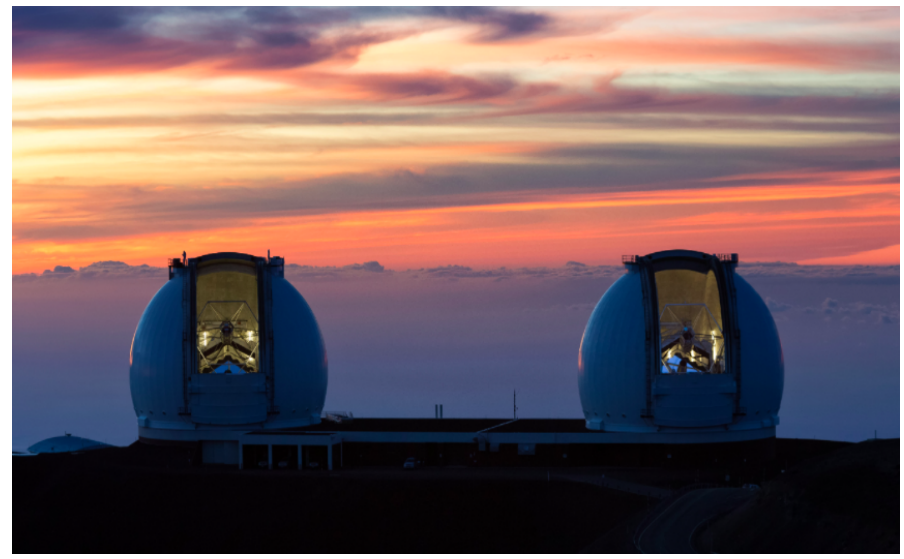

Figure 70. The Keck interferometer on top of Mauna Kea (Hawaii, USA). (c) Ethan Tweedie.

Wiener-Khinchin theorem establishes the relation between the frequency content of the point spread function of an optical instrument and its pupil plane characteristics.

\subsection{The fundamental theorem: relation between the pupil and focal planes}

The fundamental theorem that we shall demonstrate here merely stipulates that given a converging optical system which can be assimilated to the lens or to the mirror of a telescope, or of an optical interferometer, the complex amplitude distribution $a(p, q)$ of the electromagnetic field of radiation in the focal plane is the Fourier transform of the complex amplitude distribution $A(x, y)$ of the electromagnetic field in the pupil plane, i.e.

$$
a(p, q)=\int_{R^{2}} A(x, y) \exp [-i 2 \pi(p x+q y)] d x d y,
$$

or in a more compact form

$$
a(p, q)=F T[A(x, y)](p, q),
$$

with 


$$
p=\frac{x^{\prime}}{\lambda f} \quad \text { and } \quad q=\frac{y^{\prime}}{\lambda f},
$$

where $x^{\prime}, y^{\prime}$ refer to the Cartesian coordinates in the focal plane, $\lambda$ to the wavelength of the monochromatic light under consideration and $f$ to the effective focal length of the converging system.

Figure 71 represents a convergent optical system, its focal point $F^{\prime}$, its principal planes $P, P^{\prime}$ and its principal points $H$ and $H^{\prime}$. The latter degenerate with the optical center in the case of a thin lens or with the bottom of the dish in the case of a single mirror. The two orthonormal coordinate systems $(O, x, y, z)$ and $\left(F^{\prime}, x^{\prime}, y^{\prime}, z^{\prime}\right)$ make it possible to locate the input pupil plane and the image focal plane of the optical system. The term 'pupil plane' serves as the support for the definition of the vibration state at the entrance of the collector while the 'focal plane' serves as the support for the definition of the image that the collector gives of the source located at infinity. Defining the action of the collector is thus to establish the transformation that it operates on the radiation between these two planes.

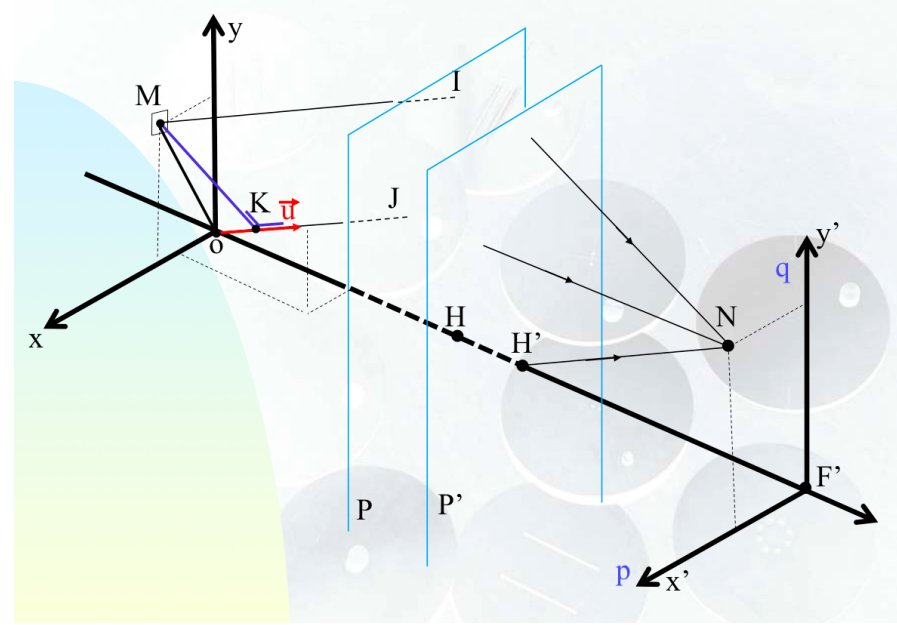

Figure 71. Fourier transform by a focusing optical system represented by its main planes $P$ and $P^{\prime}$. For the case of a thin lens, the latter would be degenerated into a single plane passing through its center.

The hypotheses underlying this theorem are:

H1. The optical system is free from any geometric aberration.

$\mathrm{H} 2$. The edges of the diaphragm do not disturb the electromagnetic field of radiation, that is to say that the diaphragm behaves as an "all (1) or nothing (0)" function with respect to this field. This is equivalent to assume that the dimensions of the collecting aperture(s) are large with respect to the wavelength of the light.

H3. No disturbance, other than those imposed by the optical system, intervenes between the pupil and the focal planes. The optical elements are thus assumed to be perfectly transparent or reflective.

H4. The light source is located at an infinite distance from the optical system and can thus be considered to be point-like.

H5. The disturbances occurring between the source and the pupil plane are weak and have very long evolution times relative to the period (i.e. $T=1 / v=\lambda / c$ ) of the radiation. 
H6. The radiation is monochromatic and has a fixed polarization plane.

Theorem statement:

Within a multiplicative coefficient of the variables, the amplitude distribution in the focal plane is the Fourier transform of the amplitude distribution in the pupil plane.

\section{Demonstration:}

Consider the different points $(x, y)$ of the pupil plane. H6 (i.e. the previous hypothesis 6 ) makes it possible to represent the electrical component of the electromagnetic field by the real part of the vibration distribution

$$
A(x, y) \exp (i 2 \pi v t)
$$

with the very general representation of the expression of the complex amplitude $A(x, y)$

$$
A(x, y)=\mathcal{A}(x, y) \exp (i \Phi(x, y)) P_{0}(x, y),
$$

where $\mathcal{A}(x, y)$ and $\Phi(x, y)$ represent the amplitude and phase of the electric field and $P_{0}(x, y)$ the input pupil function which is 1 inside the pupil and 0 outside (in agreement with $\mathrm{H} 2$ and $\mathrm{H} 3)$.

In agreement with the Huygens-Fresnel principle, we will consider in the following that every point reached by a wave can be considered as a secondary source re-emitting a vibration with the same amplitude, the same frequency $v$, the same polarization and the same phase (within a constant phase shift of $\pi / 2$ ) as those of the incident vibration at this point. The point $N\left(x^{\prime}, y^{\prime}\right)$ of the focal plane will thus receive vibrations emitted by all the points of the pupil plane. The laws of geometrical optics, deduced from the Fermat principle, make it possible to write that the rays which, after the optical system, converge at the point $N$ of the image focal plane, were, before the optical system, parallel to $H^{\prime} N$. Having assumed that the source is at infinity (in agreement with H4), the amplitude will be preserved between the pupil plane and the focal plane. From the point $M(x, y)$ of the pupil plane, the point $N\left(x^{\prime}, y^{\prime}\right)$ of the focal plane will thus receive the vibration

$$
A(x, y) \exp (i 2 \pi v t+i \Psi) .
$$

Let us take as the zero phase shift reference that of the ray passing through the point $O$ along the direction $O J N$. The phase shift $\Psi$ can then be expressed using the difference between the optical paths

$$
\delta=d(M I N)-d(O J N),
$$

where $d()$ refers to the distance along the specified path, and the relation

$$
\Psi=2 \pi \delta / \lambda
$$

If the point $K$ corresponds to the orthogonal projection of $M$ onto $O J, M$ and $K$ belong to the same wave plane which, after the optical system, will converge at the $N$ point of the focal plane.

The Fermat principle, according to which the optical path between a point and its image is constant (rigorous stigmatism) or extremum (approximate stigmatism) makes it possible to write that the difference in optical path $(M I N)-(K J N)$ behaves in the neighborhood of zero as an infinitely small second order with respect to the $d(I, J)$ and thus also with respect to $d(O, M)$ and $d(O, K)$, which are of the same order as $d(I, J)$. As a result (see Fig. 71),

$$
\delta=-d(O, K)=-|(\mathbf{O M} \mathbf{u})|,
$$


$\mathbf{u}$ designating the unit vector along the direction $H^{\prime} N$ and $(\mathbf{O M} \mathbf{u})$ the scalar product between the vectors $\mathbf{O M}$ and $\mathbf{u}$. If the angle that $H^{\prime} N$ makes with the optical axis is small, the vector of components $\left(x^{\prime} / f, y^{\prime} / f, 1\right)$ is the vector director of $H^{\prime} N$ and has a norm close to 1 (at first order because $\left.f>>\left|x^{\prime}\right|,\left|y^{\prime}\right|\right)$. Moreover, $\mathbf{O M}$ has for components $(x, y, 0)$. Using Eq. (133) in (132), the expression (130) becomes

$$
A(x, y) \exp \left(i 2 \pi v t-x x^{\prime} / \lambda f-y y^{\prime} / \lambda f\right) .
$$

Choosing as new variables in the focal plane those defined in (127), we get

$$
A(x, y) \exp (-i 2 \pi(x p+y q) \exp (i 2 \pi v t) \text {. }
$$

The resulting vibration at the point $N$ will be the resultant of the vibrations transmitted towards $N$ by all the points of the pupil plane.

The equi-phase wave surfaces which reach the pupil plane are not planes if the radiation has been disturbed between the source and the entrance pupil. But the hypotheses $\mathrm{H} 4$ and $\mathrm{H} 5$ make it possible to affirm that the pupil plane is spatially coherent, that is to say that at the time scale of the vibration periods, the relative phase shift of its different points is constant. Consequently, to calculate the resulting vibration at the point $N(p, q)$ of the focal plane, it is necessary to sum the amplitudes that $N$ receives from the different points of the pupil plane. The amplitude distribution $a(p, q)$ in the focal plane then becomes

$$
a(p, q)=\int_{R^{2}} A(x, y) \exp (-i 2 \pi(x p+y q) d x d y,
$$

that is, the complex amplitude distribution in the focal plane $a(p, q)$ is the Fourier transform of the complex amplitude distribution $A(x, y)$ in the pupil plane, i.e.

$$
a(p, q)=F T[A(x, y)](p, q) .
$$

\subsubsection{Applications of the fundamental theorem: the case of a single square aperture}

Considering first the case of a single square aperture as depicted in Fig. 72 (left) and a pointlike source perfectly located at zenith, i.e. the plane wavefronts arrive parallel to the aperture with a constant and real amplitude $A(x, y)=A_{0}$, we find that the calculation of the amplitude in the focal plane is very simple

$$
a(p, q)=A_{0} F T[\Pi(x / a) \Pi(y / a)](p, q) .
$$

Making use of the separation of the variables $x, y$ and of the relation (107), Eq. (126) successively transforms into

$$
\begin{gathered}
a(p, q)=A_{0} F T[\Pi(x / a)](p) F T[\Pi(y / a)](q), \\
a(p, q)=A_{0} a^{2} \frac{\sin (\pi a p)}{\pi a p} \frac{\sin (\pi a q)}{\pi a q} .
\end{gathered}
$$

This is the impulse response, in amplitude, for a square pupil and in the absence of any external disturbance. Adopting the definition (57) for the intensity of the vibrations, we find that (see Fig. 72, at right)

$$
i(p, q)=a(p, q) a^{*}(p, q)=|a(p, q)|^{2}=i_{0} a^{4}\left[\frac{\sin (\pi a p)}{\pi a p}\right]^{2}\left[\frac{\sin (\pi a q)}{\pi a q}\right]^{2} .
$$




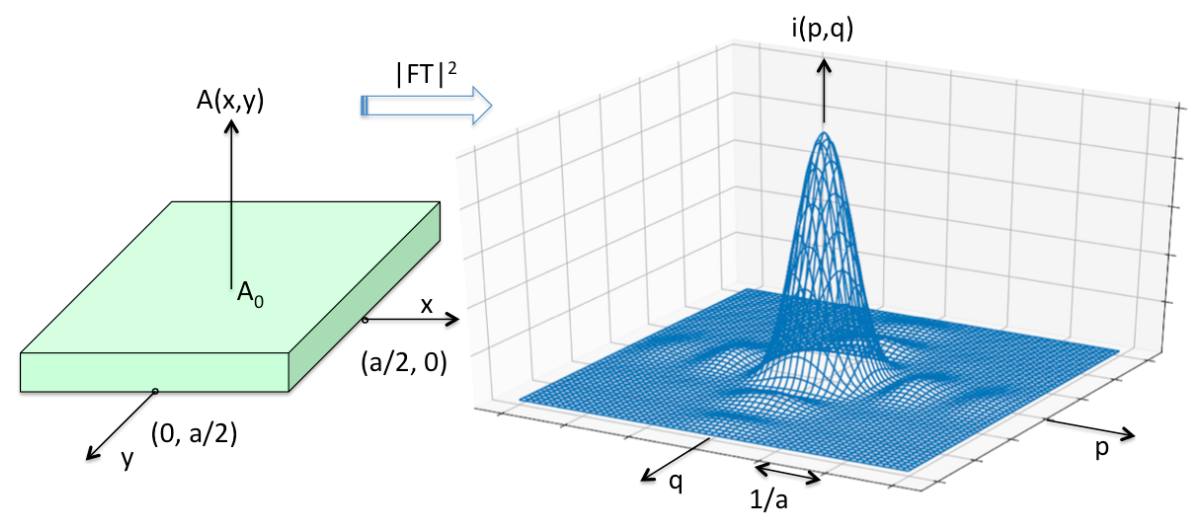

Figure 72. Complex amplitude distribution $A(x, y)$ in the plane of a single square aperture (left) and resulting response function in intensity $i(p, q)$ (right).

Defining the angular resolution $\Phi$ of an optical system as being the angular width of the response function in intensity inside the first minima, we obtain for the values of $\pi p a= \pm \pi$ (resp. $\pi q a= \pm \pi$ ), i.e. $p= \pm 1 / a($ resp. $q= \pm 1 / a$ ) and with the definition (127) for $p, q$

$$
\begin{gathered}
\frac{x^{\prime}}{\lambda f}= \pm \frac{1}{a} \quad\left(\text { resp. } \quad \frac{y^{\prime}}{\lambda f}= \pm \frac{1}{a}\right), \\
\Phi=\frac{\Delta x^{\prime}}{f}=\frac{\Delta y^{\prime}}{f}=\frac{2 \lambda}{a} .
\end{gathered}
$$

The angular resolution is thus inversely proportional to the size a of the square aperture, and proportional to the wavelength $\lambda$. Working at short wavelength with a big size aperture thus confers a better angular resolution.

Up to now, we have considered that the source $S$, assumed to be point-like and located at an infinite distance from the optical system, was on the optical axis of the instrument. Suppose now that it is slightly moved away from the zenith direction by a small angle. Let $(b / f, c / f, 1)$ be the unit vector representing the new direction of the source, the previous one being $(0,0,1)$. The plane wavefront falling on the square aperture will not have anymore a constant amplitude $A_{0}$ because each point of the pupil touched by such a wavefront will experience a phase shift given by the angle

$$
\Psi=\frac{2 \pi \delta}{\lambda}=\frac{2 \pi(x b / f+y c / f)}{\lambda}
$$

and consequently the correct expression of the complex amplitude $A(x, y)$ to be inserted in Eq. (126) becomes

$$
A(x, y)=A_{0} \Pi(x / a) \Pi(y / a) \exp \left[\frac{i 2 \pi(x b / f+y c / f)}{\lambda}\right] .
$$

Proceeding as previously, we easily find that

$$
a(p, q)=A_{0} F T\left[\Pi\left(\frac{x}{a}\right)\right]\left(p-\frac{b}{\lambda f}\right) F T\left[\Pi\left(\frac{y}{a}\right)\right]\left(q-\frac{c}{\lambda f}\right)
$$


and finally

$i(p, q)=a(p, q) a^{*}(p, q)=|a(p, q)|^{2}=i_{0} a^{4}\left[\frac{\sin \left(\pi a\left(p-\frac{b}{\lambda f}\right)\right)}{\pi a\left(p-\frac{b}{\lambda f}\right)}\right]^{2}\left[\frac{\sin \left(\pi a\left(q-\frac{c}{\lambda f}\right)\right)}{\pi a\left(q-\frac{c}{\lambda f}\right)}\right]^{2}$.

The resulting intensity response function in the focal plane is nearly the same as the one previously calculated for the case $b=0, c=0$. It is being merely translated by a linear offset $(b, c)$ in the $x^{\prime}, y^{\prime}$ focal plane and implies the invariance of the response function for a reference star that is being slightly offset from the optical axis of the system.

\subsubsection{Applications of the fundamental theorem: the case of a circular aperture}

Considering now a circular aperture with radius $R$, the complex amplitude $A(x, y)$ in the pupil plane may be represented as a circular symmetric distribution, i.e. $A(\rho, \varphi)=A_{0}$ for $\rho<R, \varphi \in[0,2 \pi]$ and $A(\rho, \varphi)=0$ for $\rho>R$ (see Figure 73, at left). We naturally expect the distribution of the complex amplitude in the focal plane to be also circular symmetric, i.e.

$$
a\left(\rho^{\prime}\right)=F T[A(\rho, \varphi)]\left(\rho^{\prime}\right) .
$$

It is here interesting to note that performing the above Fourier transform is quite alike deriving the expression of the visibility of a $2-D$ uniform circular disk star which angular diameter is $\theta_{U D}$ (see the last application in Section 4.5). We may just make use of the result (123) with appropriate changes of the corresponding variables. We easily find that

$$
a\left(\rho^{\prime}\right)=A_{0} \pi R^{2}\left[2 J_{1}\left(\frac{2 \pi R \rho^{\prime} /(\lambda f)}{2 \pi R \rho^{\prime} /(\lambda f)}\right)\right] .
$$

The resulting intensity response function is thus given by

$$
i\left(\rho^{\prime}\right)=a\left(\rho^{\prime}\right)^{2}=A_{0}^{2}\left(\pi R^{2}\right)^{2}\left[2 J_{1}\left(\frac{2 \pi R \rho^{\prime} /(\lambda f)}{2 \pi R \rho^{\prime} /(\lambda f)}\right)\right]^{2} .
$$

This is the very expression of the famous Airy disk (see Fig. 73, at right).

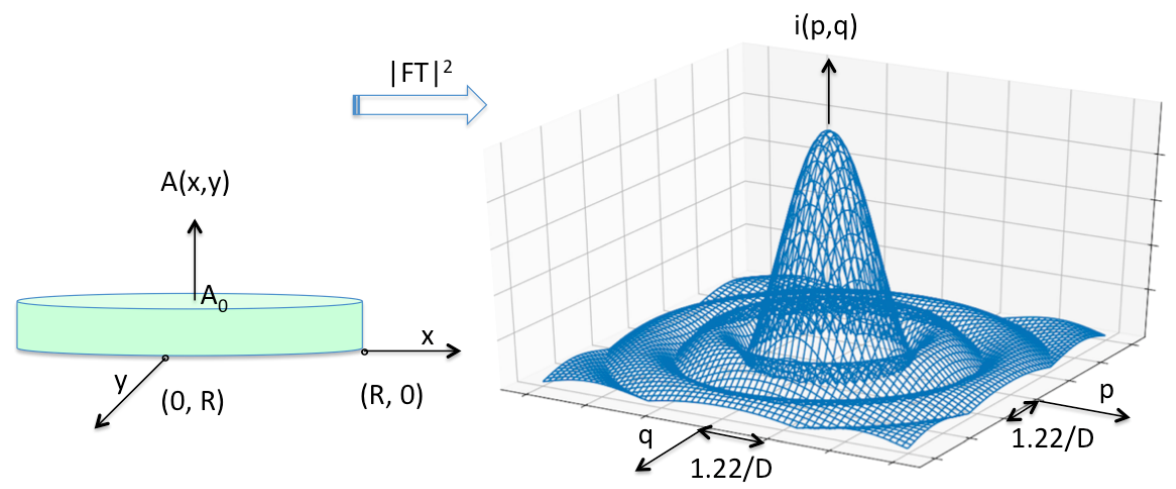

Figure 73. The Airy disk: complex amplitude distribution $A(\rho, \varphi)=A_{0}$ in the plane of a circular aperture (left) and the resulting response function in intensity $i\left(\rho^{\prime}\right)($ right). 
Knowing that the first order Bessel function $J_{1}(x)=0$ for $x \sim 3.96$, it is easy to deduce that the angular resolution $\Phi$ of a telescope equipped with a circular objective which diameter is $D=2 R$ is given by

$$
\Phi=\frac{\Delta \rho^{\prime}}{f}=\frac{2.44 \lambda}{D}
$$

\subsubsection{Applications of the fundamental theorem: the two telescope interferometer}

Figure 74 (upper left) illustrates the principle of optically coupling two telescopes. Such a system is equivalent to a huge telescope in front of which would have been placed a screen pierced with two openings corresponding to the entrance pupils of the two telescopes. The pupil function $A(x, y)$ of this system is shown in that same Figure for the case of two square apertures.

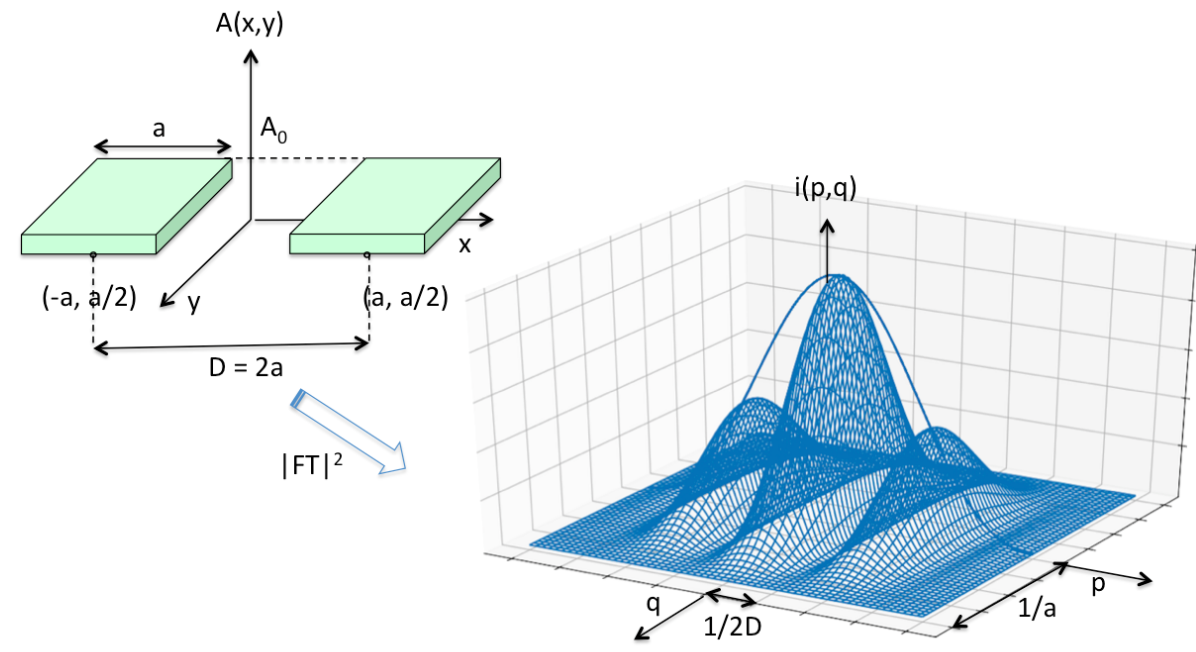

Figure 74. The two telescope interferometer: distribution of the complex amplitude for the case of two square apertures (upper left) and the corresponding impulse response function (lower right).

Let us now calculate the impulse response function $a(p, q)$ of such a system. Representing the distribution of the complex amplitude over each of the individual square apertures by means of the function $A_{0}(x, y)$ and assuming that the distance between their optical axes is $\mathrm{D}$, we find that

$$
a(p, q)=F T\left[A_{0}(x+D / 2, y)+A_{0}(x-D / 2, y)\right](p, q) .
$$

Making use of the relation (104), the previous equation reduces to

$$
\begin{gathered}
a(p, q)=[\exp (i \pi p D)+\exp (-i \pi p D)] F T\left[A_{0}(x, y)\right](p, q), \\
a(p, q)=2 \cos (\pi p D) F T\left[A_{0}(x, y)\right](p, q)
\end{gathered}
$$

and finally 


$$
i(p, q)=a(p, q) a^{*}(p, q)=|a(p, q)|^{2}=4 \cos ^{2}(\pi p D)\left\{F T\left[A_{0}(x, y)\right](p, q)\right\}^{2} .
$$

Particularizing this intensity distribution to the case of two square apertures, or circular apertures, and making use of relations (141) or (150) leads to the respective results

$$
i(p, q)=A_{0}^{2}\left(2 a^{2}\right)^{2}\left[\frac{\sin (\pi p a)}{\pi p a}\right]^{2}\left[\frac{\sin (\pi q a)}{\pi q a}\right]^{2} \cos ^{2}(\pi p D)
$$

or

$$
i\left(p, \rho^{\prime}\right)=A_{0}^{2}\left(2 \pi R^{2}\right)^{2}\left[\frac{2 J_{1}\left(2 \pi R \rho^{\prime} /(\lambda f)\right)}{2 \pi R \rho^{\prime} /(\lambda f)}\right]^{2} \cos ^{2}(\pi p D) .
$$

Figure 74 (lower right) illustrates the response function for the former case. We see that the impulse response of each individual telescope is modulated by the $\cos (2 \pi p D)$ function and that the resulting impulse response function shows consequently a more detailed structure along the $p$ axis, leading to a significantly improved angular resolution $\Phi$ along that direction. The angular width $\Phi$ of the bright central fringe is equal to the angular width separating the two minima located on its two sides. We thus find successively

$$
\begin{gathered}
\pi p D= \pm \frac{\pi}{2}, \\
p= \pm \frac{1}{2 D}, \\
\Delta p=\frac{1}{D}
\end{gathered}
$$

and making use of relation (127)

$$
\Phi=\frac{\Delta x^{\prime}}{f}=\frac{\lambda}{D}
$$

The angular resolution of the interferometer along the direction joining the two telescopes is approximately equivalent to that of a single dish telescope which diameter is equal to the baseline $D$ separating them, and not any longer to the diameter of each single telescope (see Eqs. (143) or (151)).

\subsubsection{Other types of beam recombination}

When establishing the expression for the response function of an interferometer composed of two single square or circular apertures (see Section 6.1.3, Eqs. (156-157)), we implicitly assumed that the exit pupil perfectly matched the entrance pupil (see Figs. 49, 50,53 and 75). The baseline $B$ between the two entrance pupil apertures was indeed equal to the baseline $B^{\prime}$ between the two exit pupil apertures.

This type of recombination of the two beams is referred to as the Fizeau-type or homothetic one. As we have seen in Section 3.4, Michelson and Pease have used another type of beam recombination, known as the Michelson Stellar Interferometer or still, the densified recombination type (see Fig. 76).

When the two exit beams are being superimposed, resulting in the baseline $B^{\prime}=0$, the recombination is referred to as being co-axial, or the Michelson Interferometer type (see Fig. 77). 


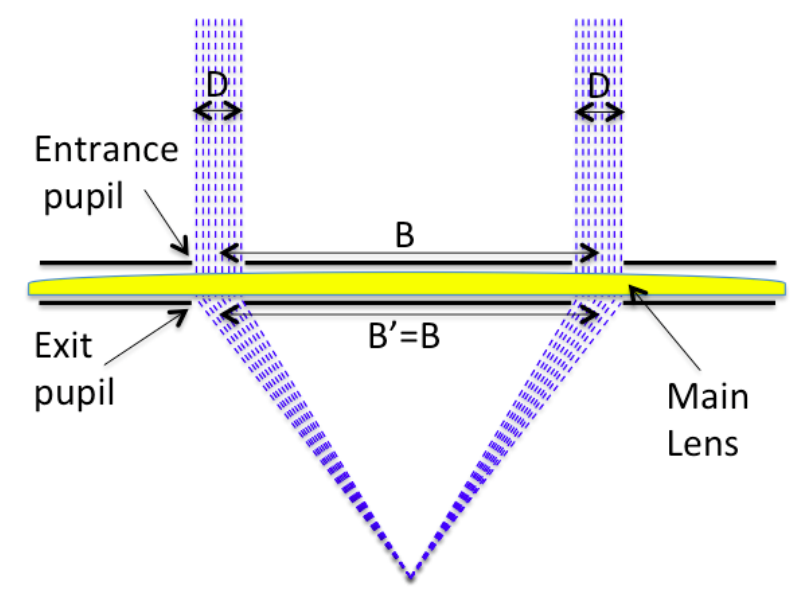

Figure 75. The two beams of light rays, represented with blue dashed lines, collected by the two entrance pupil apertures are separated by a baseline $B$ which is identical to the baseline $B^{\prime}$ between the two apertures in the exit pupil plane of the main converging lens.

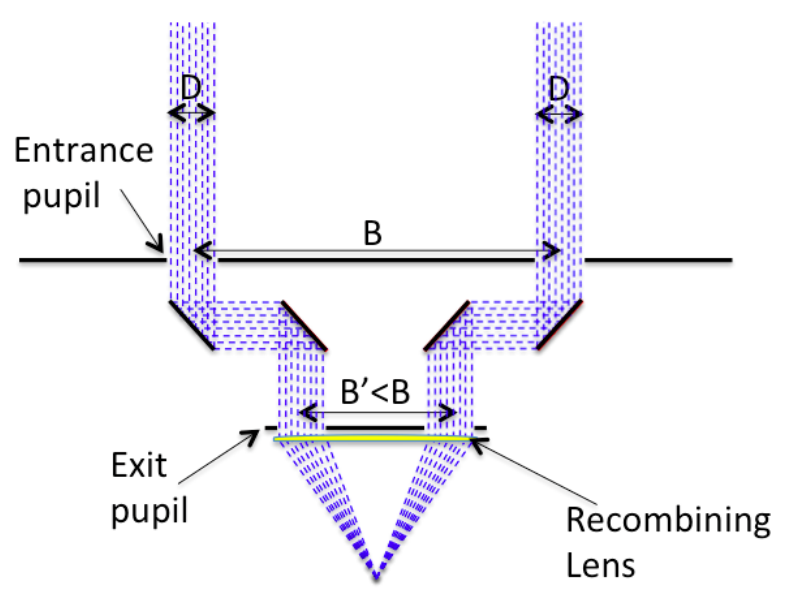

Figure 76. Sketch of the Michelson Stellar Interferometer. The baseline $B$ between the two entrance pupil apertures is much larger than the baseline $B^{\prime}$ between the two apertures in front of the recombining lens. The $45^{\circ}$ inclined black lines symbolize reflective plane mirrors. In the case of the Michelson-Pease experiment, these four mirrors were set on a $7 \mathrm{~m}$ beam just above the $2.5 \mathrm{~m}$ Wilson telescope (see Fig. 54).

A more general model of beam recombination, that includes the three previously described ones, is illustrated in Fig. 78. Two main collectors receive the light beams from a distant celestial source. While passing through the beam reducers, the beams are compressed by a magnification factor $\mathrm{M}$, corresponding to the ratio between the focal lengths of the two lenses of the focal reducers. The two compressed beams are then relayed by means of a set of 4 mirrors, just like in the Michelson Stellar Interferometer. Before entering the exit pupil 


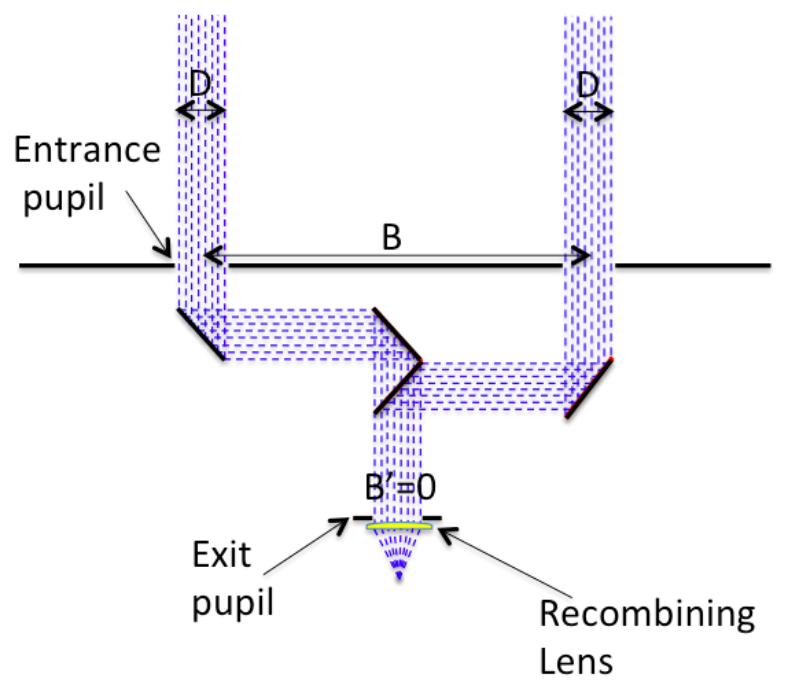

Figure 77. The Michelson Interferometer. In this case, the beam recombination is co-axial corresponding to the exit pupil baseline $B^{\prime}=0$.

of the recombining lens, their separation or baseline is $B^{\prime}<B$. To calculate the response function of such an interferometer, we just need to apply the fundamental theorem to this secondary Fizeau-type interferometer with a baseline $B^{\prime}$, taking into account the correct expression for the distribution of the complex amplitude of the electric field over the two exit pupil apertures.

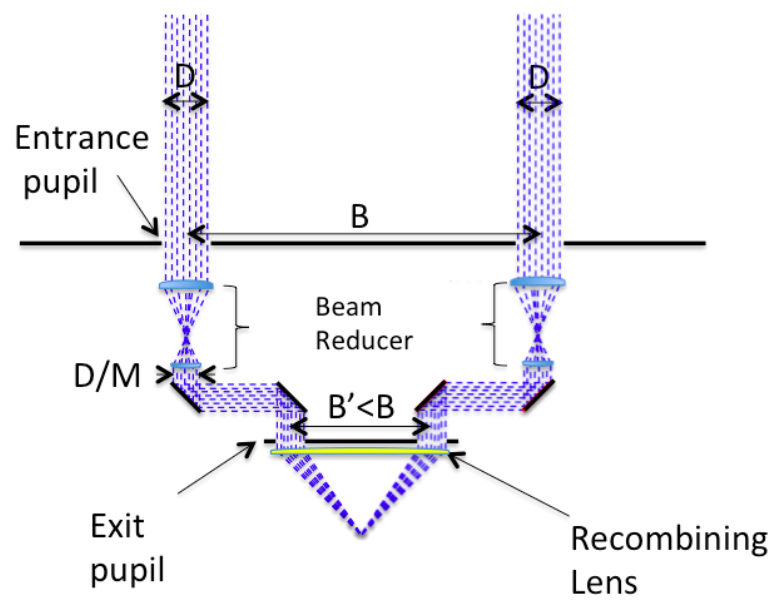

Figure 78. General case of beam recombination. The two beams of parallel light rays from a distant celestial source are first collected by two unit telescopes having a diameter $D$. The beams are then compressed by a magnification factor $\mathrm{M}$. They are subsequently relayed by a system of plane mirrors to the exit pupil of the recombining lens. At that stage, their separation (baseline) is $B^{\prime}<B$. 
Considering a point-like celestial source emitting a plane wave making an angle $\theta_{0}$ with respect to the line joining the two telescopes, the angle between the outcoming beam - compressed in size by the magnification factor $M\left(=f_{\text {in }} / f_{\text {out }}\right)$ - and the main axis of the optical system is $M \theta_{0}$ (since $\sin \left[\theta_{0}\right] \simeq \theta_{0}$, given that $\theta_{0}<<1$, see Fig. 79). The resulting complex amplitude in the focal plane of the recombining lens is along the $p$ direction, i.e. along the line joining the two exit pupil apertures (see Fig. 80 and Eq. (125))

$$
a(p)=F T\left[A_{1}(x)\right](p)+F T\left[A_{2}(x)\right](p)
$$

where $A_{1}(x)$ and $A_{2}(x)$ represent the distribution of the complex amplitude in the two exit pupil apertures along the $x$ axis.

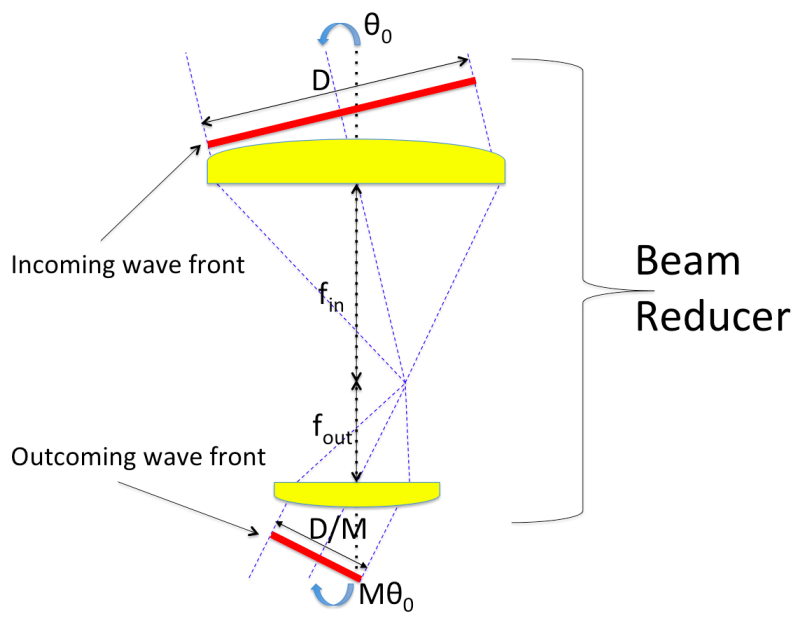

Figure 79. Propagation of an incoming plane wave from a distant celestial object with an inclination angle $\theta_{0}$ through a beam reducer. The beam size is being reduced by the magnification factor $M=$ $f_{\text {in }} / f_{\text {out }}$ while the outcoming direction of the beam has changed into $M \theta_{0}$.

We subsequently find that

$$
\begin{array}{r}
F T\left[A_{1}(x)\right](p)=\int_{-\left(B^{\prime}+D / M\right) / 2}^{-\left(B^{\prime}-D / M\right) / 2} M \exp [-2 i \pi(p x)] \exp \left[2 i \pi M \sin \left[\theta_{0}\right]\left(x-\left(B / M-B^{\prime}\right) / 2\right) / \lambda\right] d x, \\
F T\left[A_{2}(x)\right](p)=\exp [2 i \pi(d / \lambda)] \int_{\left(B^{\prime}-D / M\right) / 2}^{\left(B^{\prime}+D / M\right) / 2} M \exp [-2 i \pi(p x)] \exp \left[2 i \pi M \sin \left[\theta_{0}\right]\left(x+\left(B / M-B^{\prime}\right) / 2\right) / \lambda\right] d x .
\end{array}
$$

In this expression, we have taken into account the fact that most of existing interferometers are equipped with a delay line and we have assumed here that an extra length $d$ affects the path of the second beam. This explains the origin of the factor $\exp [2 i \pi(d / \lambda)]$ in the expression of $F T\left[A_{2}(x)\right](p)$. The limits of integration are straightforward to establish (see Fig. 80, Level 3). The presence of the factor $M$ merely accounts for the fact that when a beam is compressed, its constant amplitude is being multiplied by $\mathrm{M}$ (and the intensity $i(p)$ by $M^{2}$ in order to preserve energy conservation). The factor $\exp [-2 i \pi(p x)]$ merely accounts for the pupil-toimage relationship from Fourier optics (cf. the fundamental theorem). Since for the case of a co-phased array, the path differences affecting the arrival of the plane waves at the centres of the two apertures at Level 1 in Fig. 80 are $+\delta$ and $-\delta\left(= \pm(B / 2) \sin \left[\theta_{0}\right] \simeq \pm(B / 2) \theta_{0}\right)$, the 


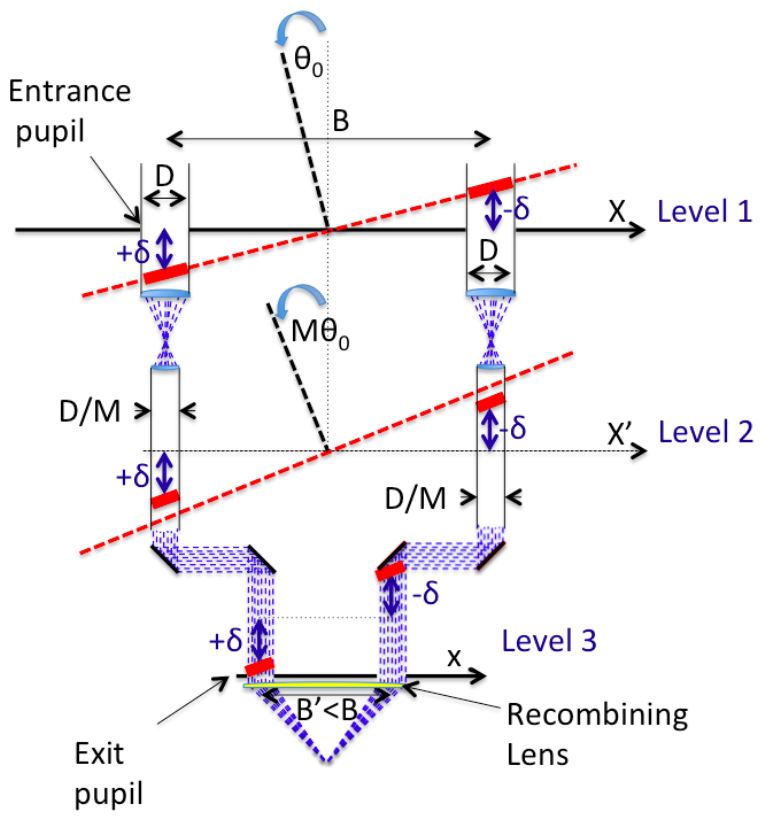

Figure 80. Propagation of an incoming plane wave from a distant celestial object with an inclination angle $\theta_{0}$ through two beam reducers (Level 1 - Level 2). When arriving in the exit pupil plane (Level 3), the delays $\pm \delta$ of the plane waves near the centres of the two apertures are the same but their inclination is now $M \theta_{0}$.

latter remain unaffected when reaching the centres of the two apertures in the exit pupil plane (Level 3). Nevertheless, their relative inclination has changed from $\theta_{0}$ to $M \theta_{0}$. Therefore, we easily understand the origin of the two factors $\exp \left[2 i \pi M \sin \left[\theta_{0}\right]\left(x-\left(B / M-B^{\prime}\right) / 2\right) / \lambda\right]$ and $\exp \left[2 i \pi M \sin \left[\theta_{0}\right]\left(x+\left(B / M-B^{\prime}\right) / 2\right) / \lambda\right]$ appearing in the two previous equations. After several successful changes of variables (see Appendix), Eq. (162) reduces to

$$
a(p)=2 D \exp [i \pi(d / \lambda)] \frac{\sin \left[(\pi D / M)\left(p-M \sin \left[\theta_{0}\right] / \lambda\right)\right]}{(\pi D / M)\left(p-M \sin \left[\theta_{0}\right] / \lambda\right)} \cos \left[\pi\left(B^{\prime} p+\left(d-B \sin \left[\theta_{0}\right]\right) / \lambda\right)\right]
$$

The corresponding expression for the intensity $i(p)=|a(p)|^{2}$ becomes

$$
i(p)=4 D^{2}\left[\frac{\sin \left[(\pi D / M)\left(p-M \sin \left[\theta_{0}\right] / \lambda\right)\right]}{(\pi D / M)\left(p-M \sin \left[\theta_{0}\right] / \lambda\right)}\right]^{2}\left[\cos \left[\pi\left(B^{\prime} p+\left(d-B \sin \left[\theta_{0}\right]\right) / \lambda\right)\right]\right]^{2} .
$$

The previous equations describe the response function of any interferometer having its entrance and exit baselines such as $0 \leq B^{\prime} \leq B$.

In the absence of an internal delay $d$, the previous expression for $i(p)$ can be rewritten as

$$
i(p)=4 D^{2}\left[\frac{\sin \left[(\pi D / M)\left(p-M \sin \left[\theta_{0}\right] / \lambda\right)\right]}{(\pi D / M)\left(p-M \sin \left[\theta_{0}\right] / \lambda\right)}\right]^{2}\left[\cos \left[\pi B^{\prime}\left(p-B \sin \left[\theta_{0}\right] /\left(B^{\prime} \lambda\right)\right]\right]^{2} .\right.
$$

Some nice features become outstanding: we first notice that the width of the envelope function is governed by the factor $\pi D / M$ which is related to the size of the beam after com- 
pression. The angular separation of the fringes $\left(\lambda / B^{\prime}\right)$ is essentially determined by the exit pupil baseline $B^{\prime}$. It does neither depend on the main baseline $B$ nor on the magnification (or beam compression) $M$. This last equation also reveals that for the response function to be field invariant, we must have $M=B / B^{\prime}$. In that case, the centre of the main envelope (cf. Airy disk for the case of a circular aperture) will always coincide with the central fringe peak, whatever the position $\left(\theta_{0}\right)$ of the source in the field of view.

Let us now consider the case of Fizeau-type interferometry for which we have $d=0$ (no delay line is being used) and in addition $M=1, B^{\prime}=B$, also $\sin \left[\theta_{0}\right] \simeq \theta_{0}$, Eq. (166) then reduces to

$$
i(p)=4 D^{2}\left[\frac{\sin \left[(\pi D)\left(p-\theta_{0} / \lambda\right)\right]}{(\pi D)\left(p-\theta_{0} / \lambda\right)}\right]^{2}\left[\cos \left[\pi\left(B\left(p-\theta_{0} / \lambda\right)\right)\right]\right]^{2} .
$$

Posing $\theta_{0}=b / f$ in the latter equation, we simply recover the result previously established for the case of Fizeau interferometry (see Eqs. (147) and (156)). We also note here that the response function of a Fizeau-type interferometer is field invariant.

Finally, the response function of a co-axial interferometer is easily derived by inserting the value $B^{\prime}=0$ in Eq. (165):

$$
i(p)=4 D^{2}\left[\frac{\sin \left[(\pi D / M)\left(p-M \theta_{0} / \lambda\right)\right]}{(\pi D / M)\left(p-M \theta_{0} / \lambda\right)}\right]^{2}\left[\cos \left[\pi\left(d-B \theta_{0}\right) / \lambda\right]\right]^{2} .
$$

We note here that the $\cos$ factor is only a function of $d$ and $\theta_{0}$, and not any longer of $p$.

In conclusion, we have established in this section a very general expression (see Eq. (165)) for the response function of an interferometer composed of two similar apertures separated by a baseline $B$ and which beams have been compressed by a magnification factor $M$. In the exit pupil plane, the new baseline between the two beams is $B^{\prime}\left(0<B^{\prime}<B\right)$ such that the fringe separation is essentially governed by the latter term.

\subsection{The convolution theorem}

The fundamental theorem has allowed us to take into account the finite size of the apertures of an optical system instead of considering that the apertures are made of pinholes. However, we have considered that the source is point-like. To treat the case of an extended source, we shall make use of the convolution theorem.

The convolution theorem states that the convolution of two functions $f(x)$ and $g(x)$ is given by the following expression

$$
f(x) * g(x)=(f * g)(x)=\int_{R} f(x-t) g(t) d t
$$

Figure 81 illustrates such a convolution product for the case of two rectangular functions $f(x)=\Pi(x / a)$ and $g(x)=\Pi(x / b)$ having the widths $a$ and $b$, respectively.

Every day when the Sun is shining, it is possible to see nice illustrations of the convolution product while looking at the projected images of the Sun on the ground which are actually produced through small holes in the foliage of the trees (see the illustration in Fig. 82). It is a good exercise to establish the relation existing between the observed surface brightness of those Sun images, the shape of the holes in the foliage of the trees, their distance from the ground and the intrinsic surface brightness distribution of the Sun. 


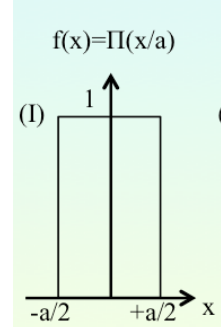

(a)

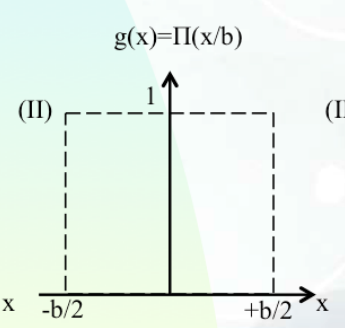

(b)

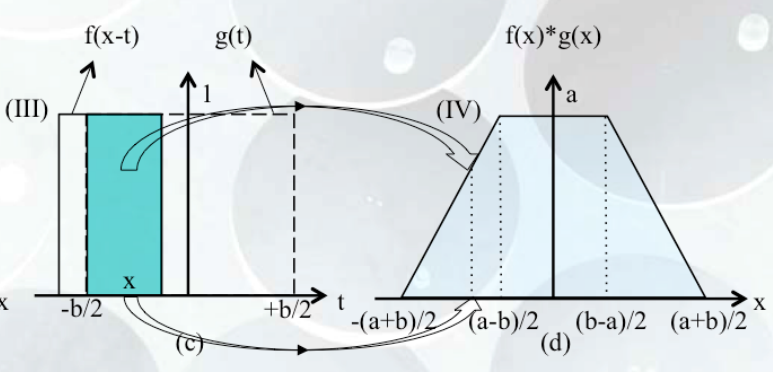

Figure 81. Convolution product of two 1-D rectangular functions. (a) $f(x)$, (b) $g(x)$, (c) $g(t)$ and $f(x-t)$. The dashed area represents the integral of the product of $f(x-t)$ and $g(t)$ for the given $x$ offset, (d) $f(x) * g(x)=(f * g)(x)$ represents the previous integral as a function of $x$.

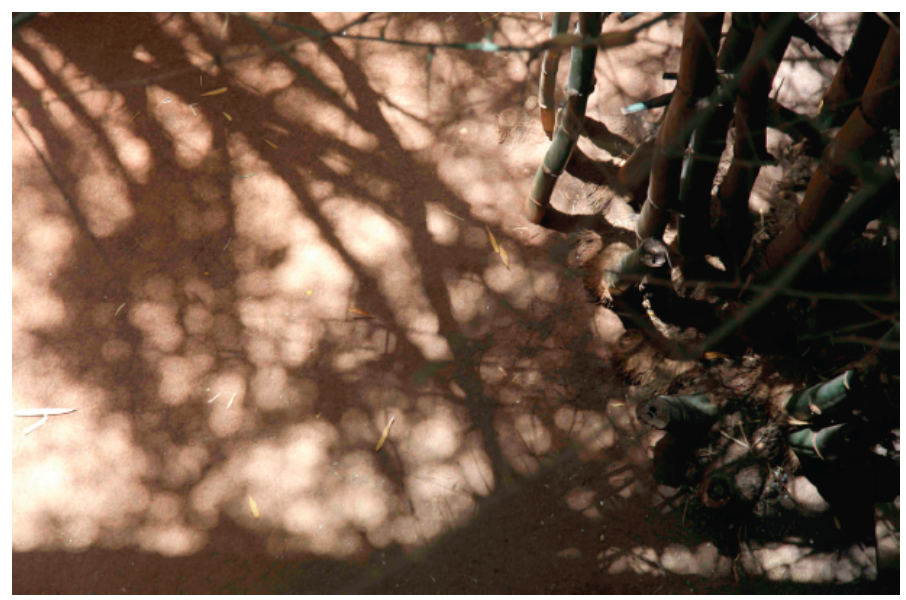

Figure 82. Projected images of the Sun on the ground actually produced through small holes in the foliage of trees (bamboo trees at IUCAA, Pune, India, June 2016). These images actually result from the convolution of the intrinsic Sun intensity distribution and the shapes of the holes in the trees.

\subsubsection{Application to the case of the two telescope interferometer}

We have previously seen that for the case of a point-like source having an intrinsic surface brightness distribution $O(p, q)=\delta(p) \delta(q)$, there results the formation of an image $e(p, q)$ in the focal plane which is the impulse response $e(p, q)=i(p, q)=|a(p, q)|^{2}$ of the optical instrument (see Eqs. (141), (150), (156), (157) for the case of a single square aperture, a single circular aperture, an interferometer composed of two square or circular apertures, respectively). Considering now an extended source represented by its intrinsic surface brightness distribution $O(p, q)$, application of the convolution theorem in two dimensions directly leads to the expression of its brightness distribution $e(p, q)$ in the focal plane of the optical system

$$
e(p, q)=O(p, q) *|a(p, q)|^{2}
$$

or more explicitly

$$
e(p, q)=\int_{R^{2}} O(r, s)|a(p-r, q-s)|^{2} d r d s .
$$

Since the Fourier transform of the convolution product of two functions is equal to the product of their Fourier transforms, we find that 


$$
F T[e(p, q)]=F T[O(p, q)] F T\left[|a(p, q)|^{2}\right]
$$

and also that the inverse Fourier transform of $F T[O(p, q)]$ leads to the result

$$
O(p, q)=F T^{-1}[F T[O(p, q)]]=F T^{-1}\left[\frac{F T[e(p, q)]}{F T\left[|a(p, q)|^{2}\right]}\right],
$$

namely, that it should be possible to recover interesting information on the intrinsic surface brightness distribution of the source $O(p, q)$ at high angular resolution provided that we get sufficient information at high frequencies in the $u, v$ plane on the object $F T[e(p, q)]$ itself as well as on a reference point-like object $F T\left[|a(p, q)|^{2}\right]$.

\subsubsection{Interferometric observations of a circular symmetric source}

Considering the case of a symmetric source around the $Y$ axis observed by means of an interferometer composed of two square apertures which size of their sides is $d$ separated along the $X$ axis by the baseline $D$, we find by means of Eqs. (141), (156) and (170) that

$$
e(p)=2 d^{2}\left(\frac{\sin (\pi p d)}{\pi p d}\right)^{2}\left[O(p) * \cos ^{2}(\pi p D)\right] .
$$

Making use of the relation $\cos (2 x)=2 \cos ^{2}(x)-1$, Eq. (174) reduces to

$$
e(p)=2 d^{2}\left(\frac{\sin (\pi p d)}{\pi p d}\right)^{2}\left[\frac{1}{2} \int_{R} O(p) d p+\frac{1}{2} O(p) * \cos (2 \pi p D)\right] .
$$

Since the function $O(p)$ is real, the previous relation may rewritten in the form

$$
e(p)=A\left[B+\frac{1}{2} \operatorname{Re}[O(p) * \exp (i 2 \pi p D)]\right]
$$

where

$$
A=2 d^{2}\left(\frac{\sin (\pi p d)}{\pi p d}\right)^{2} \text { and } B=\frac{1}{2} \int_{R} O(p) d p .
$$

Given the definition of the convolution product (cf. Eq. (169)), relation (176) can be rewritten as

$$
e(p)=A\left[B+\frac{1}{2} \operatorname{Re}\left[\int_{R} O(r) \exp (i 2 \pi(p-r) D) d r\right]\right]
$$

or

$$
e(p)=A\left[B+\frac{1}{2} \cos (2 \pi p D) F T[O(r)](D)\right],
$$

because $O(p)$ being real and even, its Fourier transform is also real. The visibility of the fringes being defined by (see Eq. (66))

$$
v=\left|\gamma_{12}(D)\right|=\left(\frac{e_{\max }-e_{\min }}{e_{\max }+e_{\min }}\right),
$$

we obtain 


$$
v=\left|\gamma_{12}(D)\right|=F T\left[\frac{O(r)}{2 B}\right](D)=F T\left[\frac{O(r)}{\int O(p) d p}\right](D) .
$$

We have thus recovered the important result (see Eq. (93), i.e. the Zernicke-van Cittert Theorem), first established for the case of two point-like apertures, according to which the visibility of the fringes is the Fourier transform of the normalized intensity distribution of the source. This result can be generalized to the case of a source that is not symmetric.

\subsection{The Wiener-Khinchin theorem}

Finally, the Wiener-Khinchin theorem allows one to easily figure out what is the space frequency content of the point spread function for a given entrance pupil of an optical instrument. We may then directly find out which information is recoverable in terms of space frequency when observing an extended source.

The Wiener-Khinchin theorem merely states that the Fourier transform of the response function of an optical system, i.e. the Fourier transform of the Point Spread Function in our case, is given by the auto-correlation of the distribution of the complex amplitude in the pupil plane. In mathematical terms, the theorem can be expressed as follows

$$
F T\left[|a(p, q)|^{2}\right](x, y)=F T[i(p, q)](x, y)=\int_{-\infty}^{+\infty} \int_{-\infty}^{+\infty} A^{*}\left(x^{\prime}+x, y^{\prime}+y\right) A\left(x^{\prime}, y^{\prime}\right) d x^{\prime} d y^{\prime}
$$

When establishing the expression (173), we wrote that the quantity $F T\left[|a(p, q)|^{2}\right]$ appearing in its denominator could be retrieved from the observation of a point-like star. The Wiener-Khinchin theorem states that it can also be retrieved from the auto-correlation of the distribution of the complex amplitude $A(x, y)$ in the pupil plane. Figure 83 illustrates the application of this theorem to the case of an interferometer composed of two circular apertures having a diameter $a$ and separated by the baseline $b$. We see that the autocorrelation of an interferometer gives access to high space frequencies.

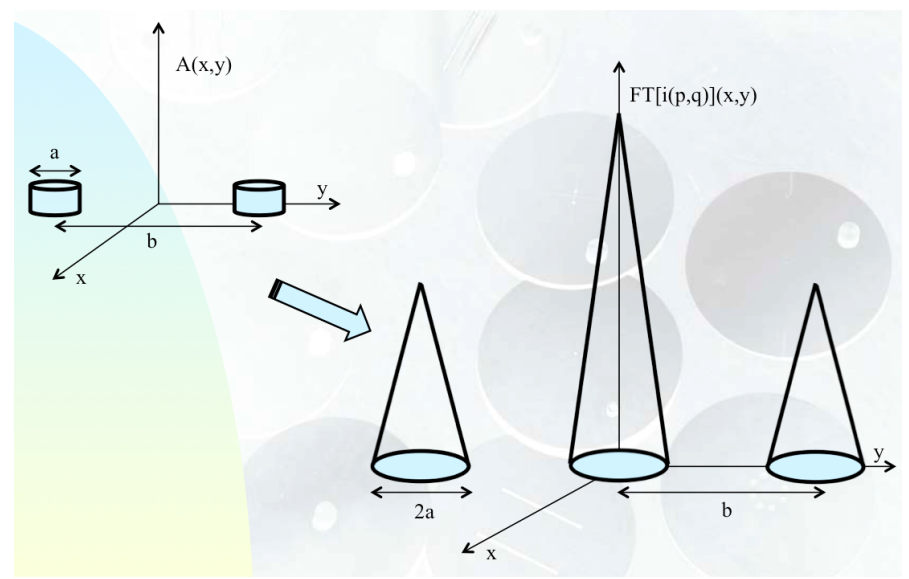

Figure 83. Diagram representing the autocorrelation function versus the space frequency, for a two telescope interferometer, each having a diameter $a$, separated by the baseline $b$.

A simple demonstration of the Wiener-Khinchin theorem (182) is given below. We may successively establish that

$$
F T[i(p, q)](x, y)=F T\left[|a(p, q)|^{2}\right](x, y)=F T\left[a^{*}(p, q) a(p, q)\right](x, y),
$$




$$
\begin{array}{r}
F T[i(p, q)](x, y)=\iint \exp [-2 i \pi(p x+q y)] \iint A^{*}\left(x^{\prime \prime}, y^{\prime \prime}\right) \exp \left[2 i \pi\left(p x^{\prime \prime}+q y^{\prime \prime}\right)\right] d x^{\prime \prime} d y^{\prime \prime} \\
\iint A\left(x^{\prime}, y^{\prime}\right) \exp \left[-2 i \pi\left(p x^{\prime}+q y^{\prime}\right)\right] d x^{\prime} d y^{\prime} d p d q,
\end{array}
$$

$$
\begin{aligned}
F T[i(p, q)](x, y)= & \iint \exp \left[\left(2 i \pi\left\{p\left[x^{\prime \prime}-\left(x^{\prime}+x\right)\right]+q\left[y^{\prime \prime}-\left(y^{\prime}+y\right)\right]\right\}\right)\right] \\
& \iint A^{*}\left(x^{\prime \prime}, y^{\prime \prime}\right) d x^{\prime \prime} d y^{\prime \prime} \iint A\left(x^{\prime}, y^{\prime}\right) d x^{\prime} d y^{\prime} d p d q
\end{aligned}
$$

and taking into account the definition (108) of the Dirac distribution

$$
F T[i(p, q)](x, y)=\iiint \int \delta\left[x^{\prime \prime}-\left(x^{\prime}+x\right)\right] \delta\left[y^{\prime \prime}-\left(y^{\prime}+y\right)\right] A^{*}\left(x^{\prime \prime}, y^{\prime \prime}\right) A\left(x^{\prime}, y^{\prime}\right) d x^{\prime} d y^{\prime} d x^{\prime \prime} d y^{\prime \prime}
$$

We finally find that

$$
\begin{array}{r}
F T[i(p, q)](x, y)=\iint A^{*}\left(x^{\prime}+x, y^{\prime}+y\right) A\left(x^{\prime}, y^{\prime}\right) d x^{\prime} d y^{\prime} d x^{\prime \prime} d y^{\prime \prime}= \\
\int_{-\infty}^{+\infty} \int_{-\infty}^{+\infty} A^{*}\left(x^{\prime}+x, y^{\prime}+y\right) A\left(x^{\prime}, y^{\prime}\right) d x^{\prime} d y^{\prime} d x^{\prime \prime} d y^{\prime \prime},
\end{array}
$$

i.e. the quoted result, namely that the Fourier transform of the impulse response function of an optical system can be represented by the autocorrelation of the distribution of the complex amplitude $A(x, y)$ in the pupil plane.

These lecture notes are based upon lectures on the same subject delivered by the author in French at the Liège University during the past ten years (see [1]). To get deeper into the field of interferometry, we highly recommend the following books: [2], [3], [4], [5].

Finally, I wish to thank the organizers of the 2017 Evry Schatzman School (Dr. N. Nardetto, Prof. Y. Lebreton, Dr. E. Lagadec and Dr. A. Meilland) for their invitation to give these lectures and for the warm hospitality and nice atmosphere in Roscoff during that event.

\subsection{Appendix}

In this appendix, we detail the calculations leading from Eqs. (162)-(163) to Eq. (164).

First of all, we proceed with the following change of variables in the expression of $F T\left[A_{1}(x)\right](p): y=-x, d y=-d x$. We then replace $y$ by $x$ and $d y$ by $d x$. Putting the factor $\exp [i \pi d / \lambda]$ in evidence, the summation of $F T\left[A_{1}(x)\right](p)$ and $F T\left[A_{2}(x)\right](p)$ leads to 


$$
\begin{array}{r}
a(p)=\exp [i \pi(d / \lambda)]\left\{\exp [-i \pi(d / \lambda)] M \exp \left[-i \pi M \sin \left[\theta_{0}\right]\left(B / M-B^{\prime}\right) / \lambda\right] .\right. \\
\int_{\left(B^{\prime}-D / M\right) / 2}^{\left(B^{\prime}+D / M\right) / 2} \exp \left[2 i \pi x\left(p-M \sin \left[\theta_{0}\right] / \lambda\right)\right] d x+ \\
\exp [i \pi(d / \lambda)] M \exp \left[i \pi M \sin \left[\theta_{0}\right]\left(B / M-B^{\prime}\right) / \lambda\right] . \\
\left.\int_{\left(B^{\prime}-D / M\right) / 2}^{\left(B^{\prime}+D / M\right) / 2} \exp \left[-2 i \pi x\left(p-M \sin \left[\theta_{0}\right] / \lambda\right)\right] d x\right\}
\end{array}
$$

and subsequently

$$
\begin{array}{r}
a(p)=M \exp [i \pi(d / \lambda)] \int_{\left(B^{\prime}-D / M\right) / 2}^{\left(B^{\prime}+D / M\right) / 2}\left\{\operatorname { e x p } \left[i \pi \left[2 x\left(p-M \sin \left[\theta_{0}\right] / \lambda\right)-\left(d+M \sin \left[\theta_{0}\right]\left(B / M-B^{\prime}\right)\right]+\right.\right.\right. \\
\exp \left[-i \pi\left[2 x\left(p-M \sin \left[\theta_{0}\right] / \lambda\right)-\left(d+M \sin \left[\theta_{0}\right]\left(B / M-B^{\prime}\right)\right] d x\right\}\right. \\
(189) \\
a(p)=2 M \exp [i \pi(d / \lambda)] \int_{\left(B^{\prime}-D / M\right) / 2}^{\left(B^{\prime}+D / M\right) / 2} \cos \left[\pi \left[2 x\left(p-M \sin \left[\theta_{0}\right] / \lambda\right)-\left(d+M \sin \left[\theta_{0}\right]\left(B / M-B^{\prime}\right)\right] d x .\right.\right.
\end{array}
$$

Let us now make use of the change of variables $z=\pi\left[2 x\left(p-M \sin \left[\theta_{0}\right] / \lambda\right)-\left(d+M \sin \left[\theta_{0}\right]\left(B / M-B^{\prime}\right)\right]\right.$ such that $d x=d z /\left[2 \pi\left(p-M \sin \left[\theta_{0}\right] / \lambda\right)\right]$, Eq. (190) then transforms into

$$
\begin{array}{r}
a(p)=\frac{2 M \exp [i \pi(d / \lambda)]}{2 \pi\left(p-M \sin \left[\theta_{0}\right] / \lambda\right)}\left\{\sin \left[\pi\left\{\left(B^{\prime}+D / M\right)\left(p-M \sin \left[\theta_{0}\right] / \lambda\right)-\left(d+M \sin \left[\theta_{0}\right]\left(B / M-B^{\prime}\right)\right) / \lambda\right\}\right]\right. \\
\left.-\sin \left[\pi\left\{\left(B^{\prime}-D / M\right)\left(p-M \sin \left[\theta_{0}\right] / \lambda\right)-\left(d+M \sin \left[\theta_{0}\right]\left(B / M-B^{\prime}\right)\right) / \lambda\right\}\right]\right\},
\end{array}
$$

and still

$$
\begin{array}{r}
a(p)=\frac{D \exp [i \pi(d / \lambda)]}{\pi D\left[p-M \sin \left[\theta_{0}\right] / \lambda\right] / M}\left\{\operatorname { s i n } \left[\pi\left\{B^{\prime}\left(p-M \sin \left[\theta_{0}\right] / \lambda\right)-\left(d+M \sin \left[\theta_{0}\right]\left(B / M-B^{\prime}\right)\right) / \lambda\right\}+\right.\right. \\
\left.\pi D\left(p-M \sin \left[\theta_{0}\right] / \lambda\right) / M\right]- \\
\left.\sin \left[\pi\left\{B^{\prime}\left(p-M \sin \left[\theta_{0}\right] / \lambda\right)-\left(d+M \sin \left[\theta_{0}\right]\left(B / M-B^{\prime}\right)\right) / \lambda\right\}-\pi D\left(p-M \sin \left[\theta_{0}\right] / \lambda\right) / M\right]\right\}
\end{array}
$$

Making use of the well known relation $\sin (A+B)-\sin (A-B)=2 \cos (A) \sin (B)$, the previous equation reduces to

$$
\begin{gathered}
a(p)=2 D \exp [i \pi(d / \lambda)] \frac{\sin \left[\pi D\left[\left(p-M \sin \left[\theta_{0}\right] / \lambda\right)\right] / M\right]}{\pi D\left[\left(p-M \sin \left[\theta_{0}\right] / \lambda\right)\right] / M} . \\
\cos \left[\pi\left\{B^{\prime}\left(p-M \sin \left[\theta_{0}\right] / \lambda\right)-\left(d+M \sin \left[\theta_{0}\right]\left(B / M-B^{\prime}\right)\right) / \lambda\right\}\right]
\end{gathered}
$$

and since $\pi\left\{B^{\prime}\left(p-M \sin \left[\theta_{0}\right] / \lambda\right)-\left(d+M \sin \left[\theta_{0}\right]\left(B / M-B^{\prime}\right)\right) / \lambda\right\}=\pi\left\{B^{\prime} p+\left(d-B \sin \left[\theta_{0}\right]\right) / \lambda\right\}$ we finally obtain 


$$
\begin{array}{r}
a(p)=2 D \exp [i \pi(d / \lambda)] \frac{\sin \left[\pi D\left[\left(p-M \sin \left[\theta_{0}\right] / \lambda\right)\right] / M\right]}{\pi D\left[\left(p-M \sin \left[\theta_{0}\right] / \lambda\right)\right] / M} . \\
\cos \left[\pi\left\{B^{\prime} p+\left(d-B \sin \left[\theta_{0}\right]\right) / \lambda\right\}\right]
\end{array}
$$

which is the same result as that quoted in Eq. (164).

\section{References}

1. J. Surdej, see https : //www.youtube.com/playlist?list = PLgbVWzVdxoUtI $23 J 1 Q E w I e K r z W M V M C 1$,

http://www.aeos.ulg.ac.be/teaching.php, https://sites.google.com/view/jeansurdej/teaching, http://www.jean-surdej.eu (2018)

2. H. Réboul, Introduction à la théorie de l'Observation en Astrophysique (Masson, 1979)

3. P. Léna, D. Rouan, F., Lebrun, F. Mignard, D., Pelat, D., Observational Astrophysics (Astronomy and Astrophysics Library, 2012)

4. A. Glindemann, Principles of Stellar Interferometry (Astronomy and Astrophysics Library, 2011)

5. D. Buscher, Practical Optical Interferometry: Imaging at Visible and Infrared Wavelengths (Cambridge University Press, 2015) 


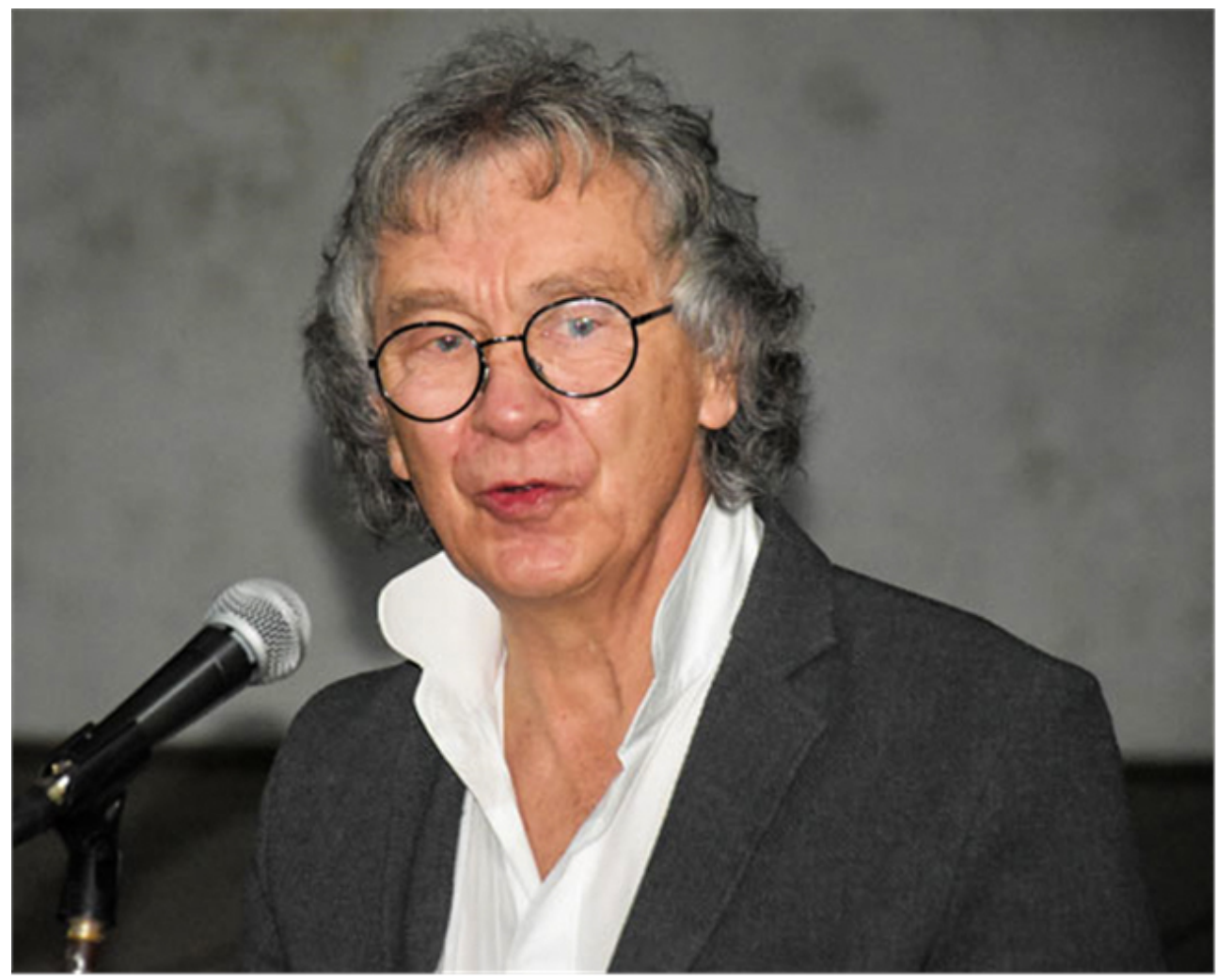

Figure 84. Jean Surdej, enlightning and entertaining us with interferometry. 


\section{Optical Long Baseline Interferometry}




\title{
Optical Long Baseline Interferometry
}

\section{Principles and Instrumentation}

\author{
${\text { Denis } \text { Mourard }^{1, *} \text { and Nicolas Nardetto }}^{1}$ \\ ${ }^{1}$ Université Côte d'Azur, OCA, CNRS, Lagrange, Parc Valrose, Bât. Fizeau, 06108 Nice cedex 02 , \\ France
}

\begin{abstract}
The principles of optical long baseline interferometry are presented firstly from the point of view of the coherence of the stellar wavefront and secondly from the point of view of the Object-Image relationship. These two complementary approaches permit to extract some important considerations for the actual implementation of this imaging technique. We also present a panorama of the currently operational optical interferometers and their most recent science programs. We conclude by a short prospective on the future steps.
\end{abstract}

\section{Introduction}

Researches in stellar physics have been, for a long time, dominated by well-established observational techniques like astrometry, photometry, polarimetry, and spectroscopy. But in all these techniques, except probably Doppler-imaging, the star is seen as a point-like source and the information on the spatial brightness distribution onto the sky is integrated. The star is considered as a source of light and the different properties of this light are sampled and analyzed. After the pioneering works at the beginning of the twentieth century by Michelson [1] and the original ideas by H. Fizeau [2], the rebirth of optical interferometry in 1974 by Antoine Labeyrie [3] has opened a completely new field of investigation: the high angular resolution permitting to reveal details at the surface or in the close environment of stars. As an imaging technique, optical interferometry requires a strict control of the quality of the optical transfer function and of the field of view. Moreover, the physical processes at work in stars dictate to combine the high spatial resolution with temporal or spectral resolution. Today, images of stellar's surface or detailed studies of the kinematic in atmospheres or disks are well established. Combined with the recent advent of exoplanet researches, and the instrumental progresses for asteroseismology or astrometry, stellar physics is clearly a central question among the top-priorities of today Astrophysics.

In this article, we will first present two complementary description of optical interferometry, either through the principles of coherence (Section 2) and those of imaging (Section 3). We then discuss, in Section 4, an important aspect of this observational technique that should be understood firstly as a direct imaging technique. Of course important practical limitations occur in reality, they will be presented in Section 5 and in Section 6. The paper will end with a panorama of the modern facilities in Section 7 and the most recent science programs in Section 8. We conclude by a few words on the future of this observational technique in Section 9.

\footnotetext{
*e-mail: denis.mourard@oca.eu
} 


\section{Angular diameter and coherence of the wavefront}

Optical interferometry is often described through the concept of interference fringes. It was indeed the driving principles expressed by $\mathrm{H}$. Fizeau [2] ${ }^{1}$ and was described on the basis of the coherence of the wavefront. The excellent book of Goodman [4] is probably one of the reference work for optical interferometry. I'm presenting first this principle with a very simple demonstration.

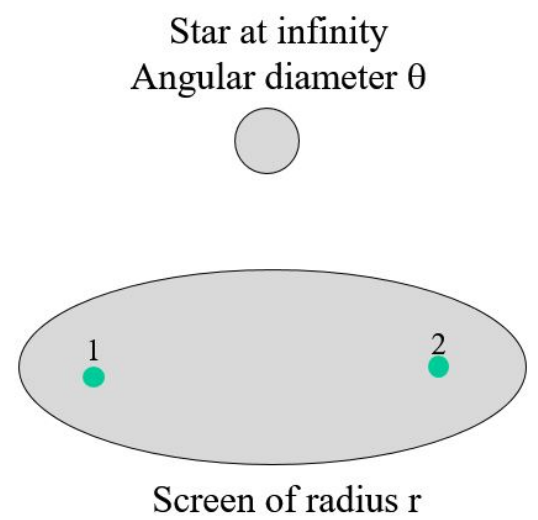

Figure 85. Schematic representation of an interferometer, from the point of view of the coherence of the wavefront.

In Fig. 85, I consider a star located at infinity and presenting an angular diameter $\theta$. This object defines a solid angle $\Omega$ defined by:

$$
\Omega=\pi\left(\frac{\theta}{2}\right)^{2} .
$$

A screen of radius $r$ and surface $S=\pi r^{2}$ intercepts the stellar wave. This defines a beam etendue $\epsilon$ that can be written as:

$$
\epsilon=S \Omega=\pi^{2} r^{2}\left(\frac{\theta}{2}\right)^{2}
$$

The principle of coherence, as defined by Goodman in his book, indicates that we can consider the wave as coherent if $\epsilon<\lambda^{2}$. This defines the so-called radius of coherence $r_{c}$ :

$$
r_{c}=\frac{\lambda}{\pi\left(\frac{\theta}{2}\right)}
$$

One can note that in the case of a star with an angular diameter $\theta=10$ mas and at a wavelength $\lambda=1 \mu \mathrm{m}$, this leads to a value of $r_{c} \simeq 13 \mathrm{~m}$. We thus understand that it exists a relation between the coherence of the wave and the angular diameter of the star. The coherence of the electromagnetic wave $\psi$ could be determined by the computation of the complex degree of

\footnotetext{
${ }^{1}$ Although the recognized reference for this discovery is dated from 1868 , it exists a note of $\mathrm{H}$. Fizeau at the French Academy of Sciences, dated from 1851, and showing that it could be possible to measure by interferometry, the apparent diameter of a source, and in particular of a star.
} 
mutual coherence $\left(\Gamma_{12}\right)$ between two points of the collecting screen separated by a distance B.

$$
\Gamma_{12}=\frac{\left|\psi_{1} \psi_{2}{ }^{*}\right|}{\sqrt{\left|\psi_{1}\right|^{2}\left|\psi_{2}\right|^{2}}} .
$$

By using the Van-Cittert Zernike theorem and the notation Õ for the Fourier Transform of the star brightness distribution, we can write the following relation:

$$
\Gamma_{12}=\frac{\tilde{O}\left(\frac{B}{\lambda}\right)}{\tilde{O}(0)} .
$$

Considering the star as a uniform disk, we finally obtain:

$$
\Gamma_{12}=\left|\frac{2 J_{1}(\pi B \theta / \lambda)}{\pi B \theta / \lambda}\right| .
$$

The definition of coherence by Goodman corresponds to the case where $\Gamma_{12}=0.5$ which corresponds to $\pi B \theta / \lambda=2$ and thus to $B=r_{c}=\frac{\lambda}{\pi\left(\frac{\theta}{2}\right)}$, which is an other way of defining the coherence $\left(\epsilon<\lambda^{2}\right)$.

This simple calculation shows that the coherence of the electromagnetic wave of stellar sources could be measured through a spatial sampling if one can access to very long baselines (B larger than 100m typically). The simplest instrumental setup dedicated to the measurement of the complex degree of mutual coherence is thus to consider the coherent addition of the two complex waves collected at point 1 and 2 with a phase shift on the second one dedicated to the necessary adjustment of the optical path between the two wave collectors. Thus we obtain the total intensity $I$ as:

$$
\begin{gathered}
I=\left|\psi_{1}+\psi_{2} e^{i \phi}\right|, \\
I=\psi_{1}^{2}+\psi_{2}^{2}+2 \psi_{1} \psi_{2} \times \cos (\phi) .
\end{gathered}
$$

Denoting $I_{i}$ the intensity of the wave at point i, we finally obtain:

$$
I=\left(I_{1}+I_{2}\right)\left(1+\frac{2 \sqrt{I_{1} I_{2}}}{I_{1}+I_{2}} \times \frac{\psi_{1} \psi_{2}{ }^{*}}{\sqrt{\left|\psi_{1}\right|^{2}\left|\psi_{2}\right|^{2}}} \times \cos (\phi)\right) .
$$

The term with the cosinus function represents, if one introduces variations of $\phi$ either by temporal or spatial sampling, a modulation in the measured intensity, which is also called interference fringes. The amplitude of the modulation is defined by the factor in front of the cosinus. It contains two parts: the photometric one $\left(\frac{2 \sqrt{I_{1} I_{2}}}{I_{1}+I_{2}}\right)$ and the coherence one $\left(\frac{\psi_{1} \psi_{2}{ }^{*}}{\sqrt{\left|\psi_{1}\right|^{2}\left|\psi_{2}\right|^{2}}}\right)$ where we recognize $\Gamma_{12}$ the complex degree of mutual coherence of the two collected waves.

These principles are mainly valid whatever the wavelengths. But many other aspects have to be taken into account, such as the spectral and temporal coherence of the electromagnetic waves. Again at $\lambda=1 \mu \mathrm{m}$, considering a spectral width $\Delta \lambda=0.1 \mu \mathrm{m}$ leads to a coherence time $t_{c}=3.10^{-14} \mathrm{~s}$ using the relation between the coherence time and the frequency domain $\left(t_{c} \times \Delta f=1\right)$. At larger wavelengths and with very fast detectors, it is possible to directly record the electromagnetic waves at two different locations and to correlate them, later, into a computer. This is usually done un radio-astronomy at millimeter and centimeter wavelengths 
for Very Long Baseline Interferometry or, by reducing the spectral width and generating beating waves with a local oscillator, through what is called heterodyne interferometry. It could also be shown that the coherence of the electromagnetic wave could be extracted from the correlation between the fluctuation of intensities at the two different locations [5]. In this paper we only consider the case of direct interferometry in the optical domain, which means that we use detectors sensitive to the intensity of the electromagnetic wave recording the intensity resulting from the coherent addition of the complex waves.

As a conclusion of this section, we see that we have indeed a way to measure complex degrees of mutual coherence of stellar waves allowing us to sample the Fourier transform of brightness distributions at very high spatial frequencies. We will see in Section 6 how this method is now implemented in reality but before coming into the instrumental part of this technique an other point of view is also very important for a correct understanding of this observing technique.

\section{Angular diameter and Object-Image relationship}

Astronomers have developed optical interferometry in order to improve the resolving power of the telescopes. Indeed image formation in a telescope is a standard diffraction problem and it is known for a long time that an image $I(\vec{\alpha})$ is obtained as the convolution of the brightness distribution of the source $O(\vec{\alpha})$ by the point spread function of the optical device $H(\vec{\alpha})$.

$$
I(\vec{\alpha})=O(\vec{\alpha}) \otimes H(\vec{\alpha})
$$

When this convolution relation is translated into the Fourier domain, it shows that the spatial frequency spectrum of an image $\widetilde{I}(\vec{u})$ is the spatial frequency spectrum of the object $\widetilde{O}(\vec{u})$ filtered by the optical transfer function $\widetilde{H}(\vec{u})$ of the optical device.

$$
\widetilde{I}(\vec{u})=\widetilde{O}(\vec{u}) \times \widetilde{H}(\vec{u})
$$

Thanks to the diffraction principle, it could be easily shown (see the paper by J. Surdej in this book) that the modulus of the optical transfer function, called the modulation transfer function (MTF), is equal to the autocorrelation of the pupil function, defining the entrance plane of the optical device.

$$
|\widetilde{H}(\vec{u})|=A C[P(\lambda \vec{u})]
$$

In the case of a monolithic telescope of diameter $D$, the MTF acts as a low pass filter transmitting the spatial frequencies of the object brightness distribution up to $D / \lambda$ (see Fig. 86). This corresponds to what is usually called the diffraction limit $\lambda / D$ of the telescope. We do not consider here the perturbations induced by the atmosphere and we just consider the ideal case of a perfect optical instrument.

In the case of an interferometer with two telescopes of diameter $D$ and separated by a vector $\vec{B}$, the support of the MTF (also called the (u,v) plane), is made of a low frequency peak of extent $\pm D / \lambda$ and two high frequency peaks of extent $\pm D / \lambda$ and located at $\pm \vec{B} / \lambda$ (see Fig. 86). The interferometer acts thus as a high frequency band pass filter, allowing to reach information at a resolution of $\lambda /|\vec{B}|$. 

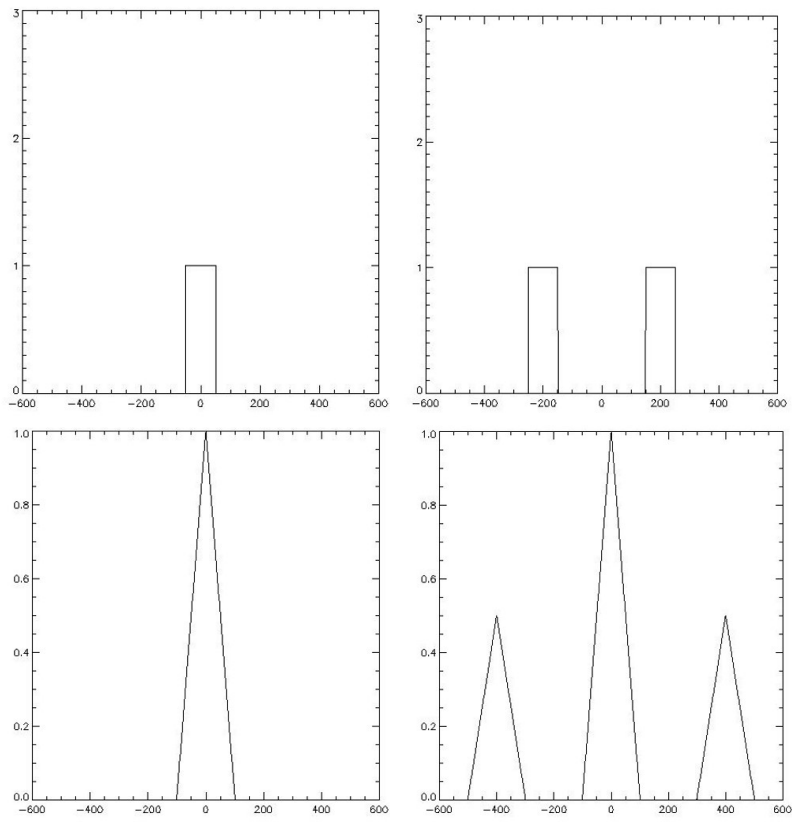

Figure 86. Modulation Transfer Function (bottom row) corresponding to the pupil (top row) of a single telescope (left) and of a 2-telescope interferometer (right). The units are arbitrary.

In the general case, the $(\mathrm{u}, \mathrm{v})$ plane (support of the MTF) is a function of the input baselines, of the latitude of the observatory, of the target coordinates, of the wavelength and of the time (because of the earth rotation). The (u,v) plane coverage defines the sampling of the Fourier transform of the object brightness distribution.

The properties of the image obtained directly at the focus of an interferometer clearly depend on the (u,v) plane coverage but it can also be shown [6] that the beam combination scheme plays also an important role in that domain. I refer the reader to the important papers published in that domain [7-10]. As an illustration I present in Fig. 87, some examples of $(\mathrm{u}, \mathrm{v})$ plane coverage and point spread function for different kind of optical interferometers.

Currently, no interferometer is working in a direct imaging scheme except the Large Binocular Telescope in its interferometric mode. The limitations of coherence for ground based projects in the optical domain are clearly difficult to overcome. Progresses are being made in that direction but for the moment, imaging at high angular resolution, is not working directly at the focus of the interferometer. Instead, astronomers are using the $(\mathrm{u}, \mathrm{v})$ plane coverage to sample the Fourier transform of the brightness distribution and then to reconstruct images. This method has made great progresses in the recent years. The quality of the reconstructed images highly depends of the $(\mathrm{u}, \mathrm{v})$ plane coverage and of the a priori information (regularization constraints) introduced in the reconstruction algorithm. I do not intend to describe this method in the present paper and I refer the reader to the practical sessions of the school. 


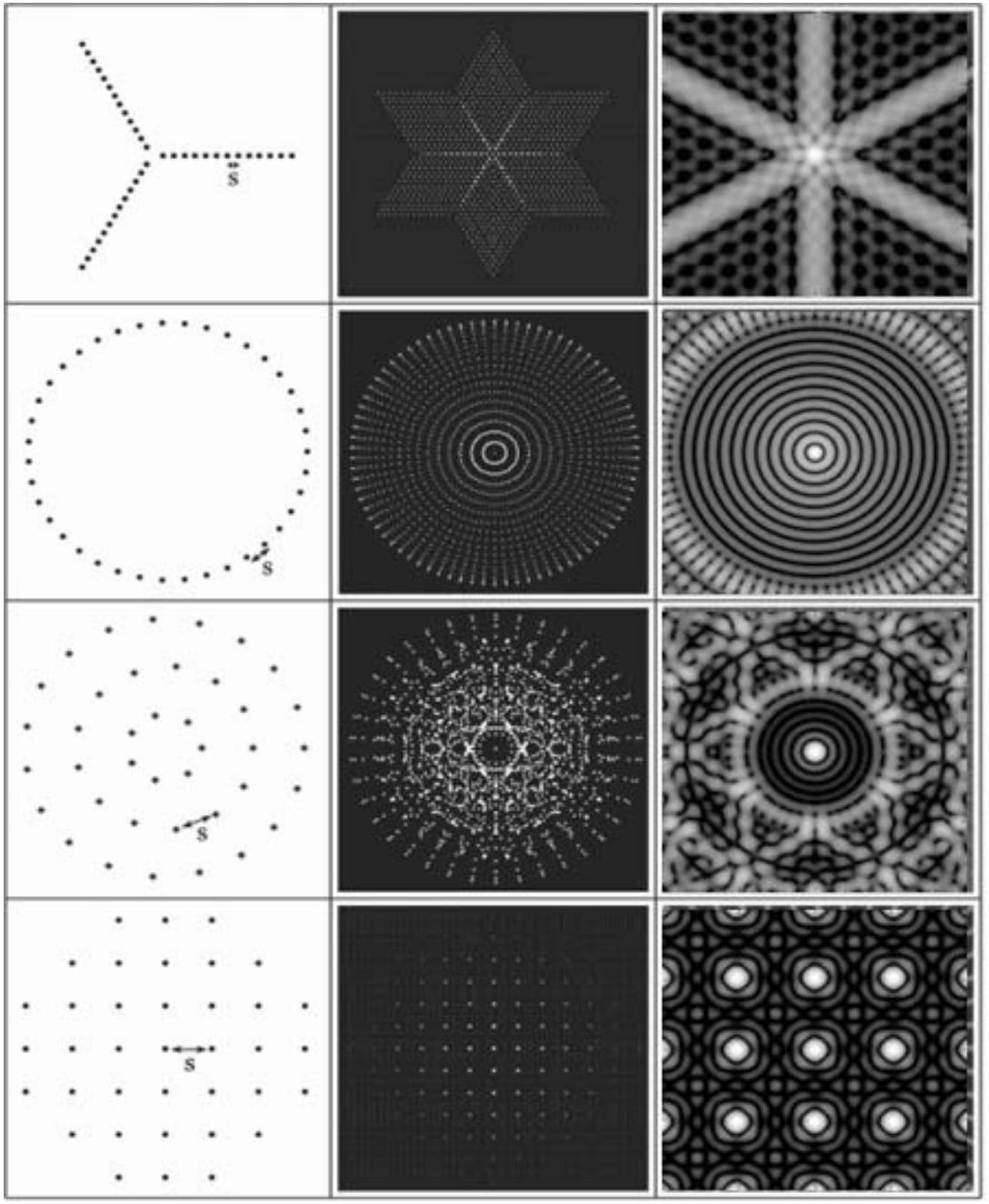

Figure 87. Examples of (u,v) plane coverage (middle column, autocorrelation of the pupil) and of the corresponding point spread function (right column, modulus of the Fourier transform of the autocorrelation of the pupil) for four different input pupil (left column) configurations.

I will conclude this section by giving some general considerations about image reconstruction with an interferometer. First of all, an interferometer made of $N$ telescopes produces $N(N-1) / 2$ baselines and thus samples $N(N-1) / 2$ frequencies in the Fourier transform of the brightness distribution of the object. We thus have a problem with $N(N-1) / 2$ complex unknowns. 


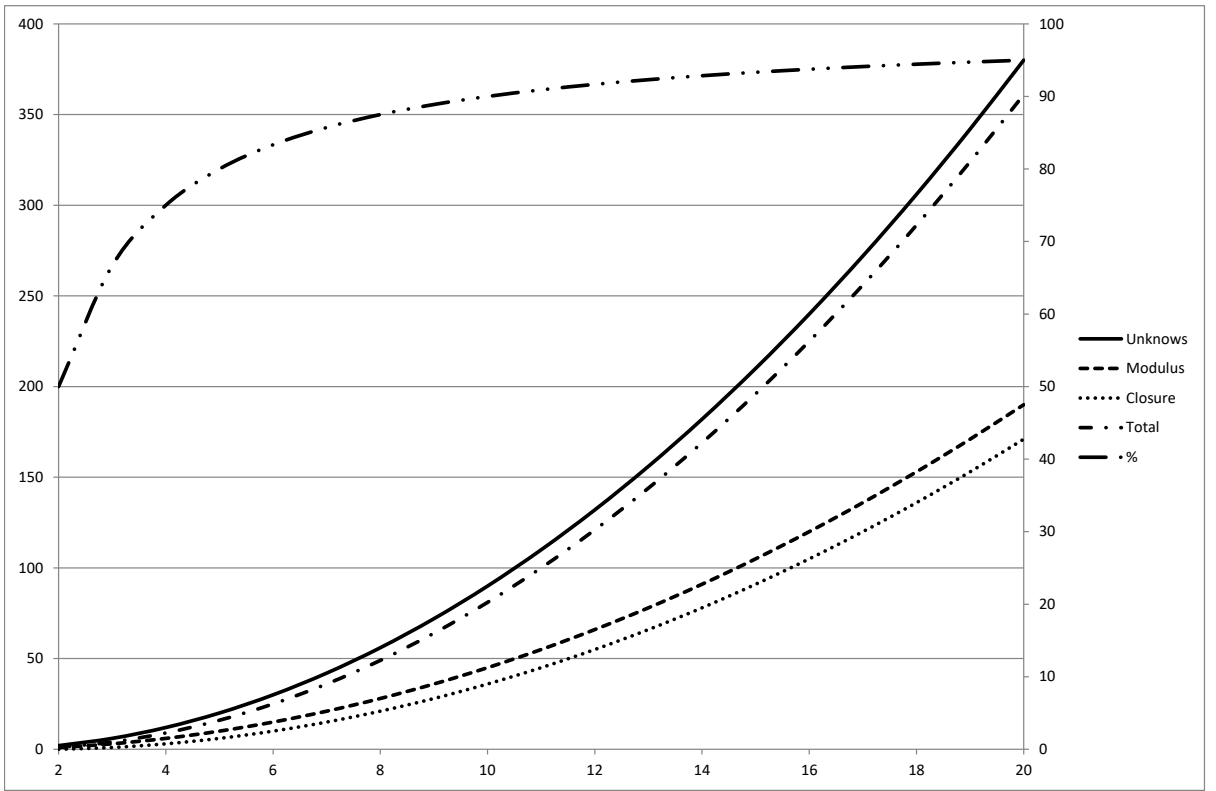

Figure 88. Number of unknowns, of modulus and closure phase measurements (left scale) as a function of the number of telescopes. The dark curve represents the percentage (right scale) of information measured as a function of the number of telescopes.

We have already indicated that the limitations of ground based interferometers are dominated by the phase effects introduced by the atmospheric turbulence. If it is easy to measure the modulus of the Fourier transform over the $N(N-1) / 2$ points, the phase measurements are however highly corrupted by the turbulence. As in radio interferometry, astronomers overcome this difficulty by computing closure phase measurements over triplets of apertures. It can be shown easily that the atmospheric phase terms are removed in the sum of the phase of three interference fringes over any triplet of telescopes. Thus closure phase measurements (see explanations in Section 6) give us access to $(N-1)(N-2) / 2$ additional measurements. With this in hand, we understand that the problem is not well constrained because the number of unknowns is always larger than the number of measurements. A representation of these numbers is presented on Fig. 88.

\section{A direct imaging technique}

We concluded the previous section on the idea that, ideally, an interferometer may have the same imaging properties as a single giant telescope. If this is true from the point of view of the optical system, in practice the encoding of the information is quite different and to reach this ultimate goal the implementation of interferometers should respect a certain number of conditions and some restrictions appear. A schematic representation of two generic configurations of interferometers is given in Fig. 89. The first case (A) represents what is usually 
called the Fizeau combination. The two segments are really identical to elements of the giant parabola and if the number of sub-elements is increased the imaging properties of the diluted parabola approach the ones of the giant mirror. The transfer function is not as rich as for the full pupil but the main property of the highest angular resolution could be easily preserved.

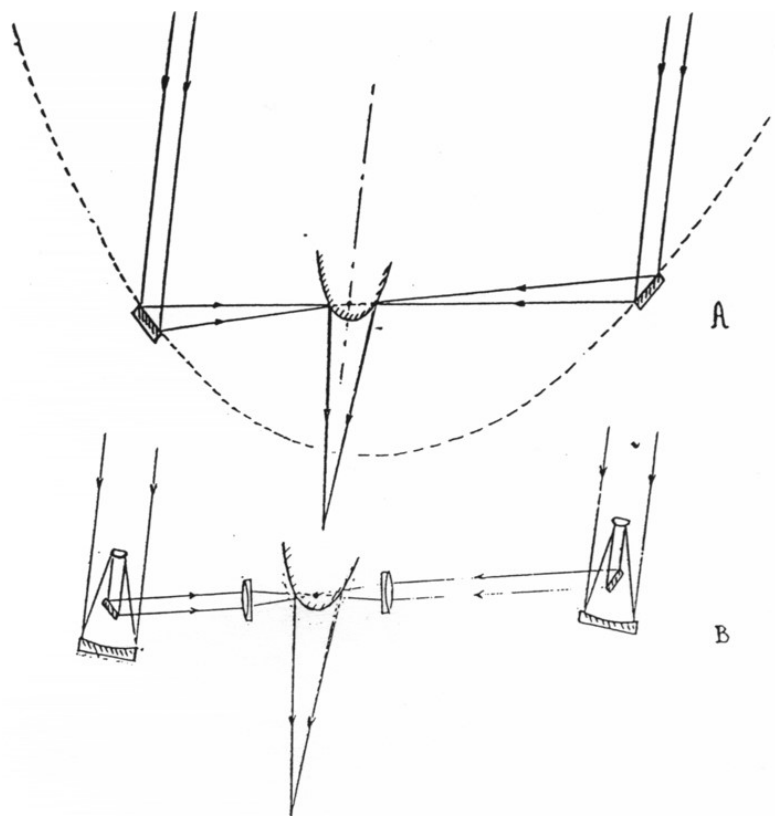

Figure 89. Schematic representation of an interferometer. A: the Fizeau type, B: the Michelson type.

This figure helps us to understand that the same formalism of image formation is in action when considering an interferometer and not only of single telescope. This property of convolution has been studied with many details by Labeyrie [6] and by Tallon\&Tallon-Bosc [11]. These works have generated the idea that an interferometer could work as a direct imager and not only as an aperture-synthesis experiment sampling the spatial frequency plane and allowing an, a posteriori, image reconstruction. A direct image could be obtained at the common focus of the interferometric array. This was known in the Fizeau-like mode but this one is not efficient as the sampling is far from being optimized. The pupil-densification scheme and the hypertelescope principle have permitted to develop a new way of thinking the interferometers, just as direct-imaging devices. In Fig. 90, I present the Fizeau Point Spread Function for different pupil configurations. If we can easily understand that with an increasing number of sub pupils, the point spread function is progressively converging towards the point spread function of the large monolithic telescope, we also identified that the interferometric Fizeau process generates also a large number of bright side peaks in the point spread function, degrading therefore the image quality. This poor imaging quality can be overcome by using the principle of pupil densification as this is done implicitly in fact in almost all aperture synthesis interferometers, and even in the Michelson experiment presented in Fig. 91. Thanks to this important aspect of the beam combination in an interferometer, it is possible to have a fixed sampling of fringes whatever the entrance pupil. The pupil densification adds an important constraint with respect to the standard pupil reconfiguration of current interferometers: the geometry of the input and output pupil has to be respected but each output pupil could be magnified to improve the direct imaging properties. Of course there is a price to pay because 
the convolution relation is broken in this densification process. The drawback is a reduction of the field of view but it can be shown that in the direct imaging field, a pseudo relation of convolution exists and that a true direct image is indeed formed. The properties of this direct imaging scheme (Clean Field, Direct Imaging Field, Interferometric Field, encircled energy) have been studied in many details by different authors [7, 8]. I present in Fig.92 various possible interferometric arrays and in Fig. 93 their imaging properties in a densified combination scheme.

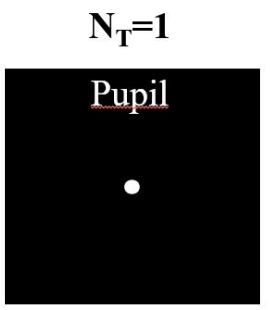

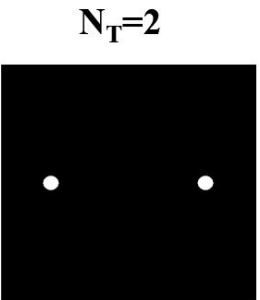

$\mathbf{N}_{\mathrm{T}}=\mathbf{3}$
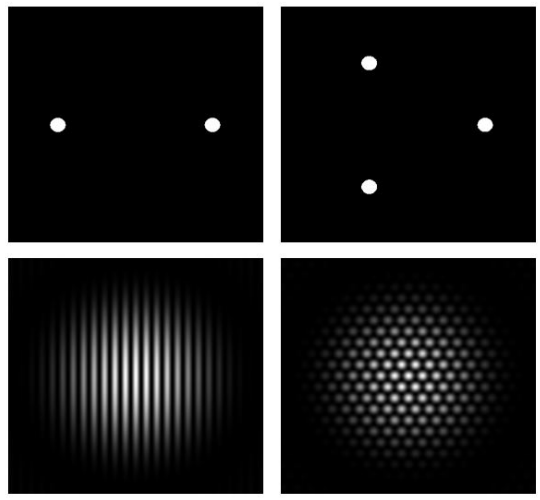

$\mathbf{N}_{\mathrm{T}}=8$
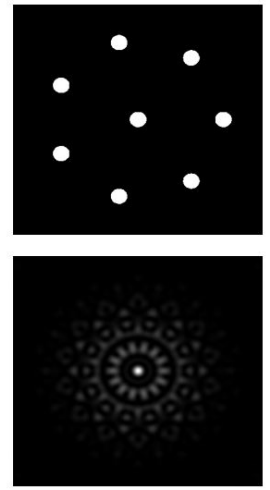

$\mathbf{N}_{\mathrm{T}}=1$
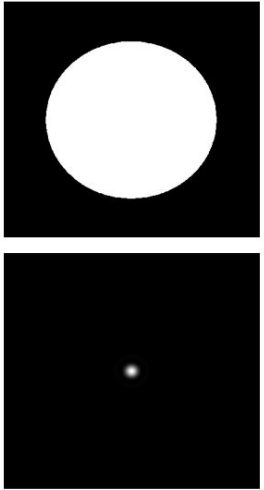

Figure 90. The upper row presents different pupils, from a small telescope to a large telescope, and with three different interferometric pupils. The bottom row presents the corresponding point spread function.

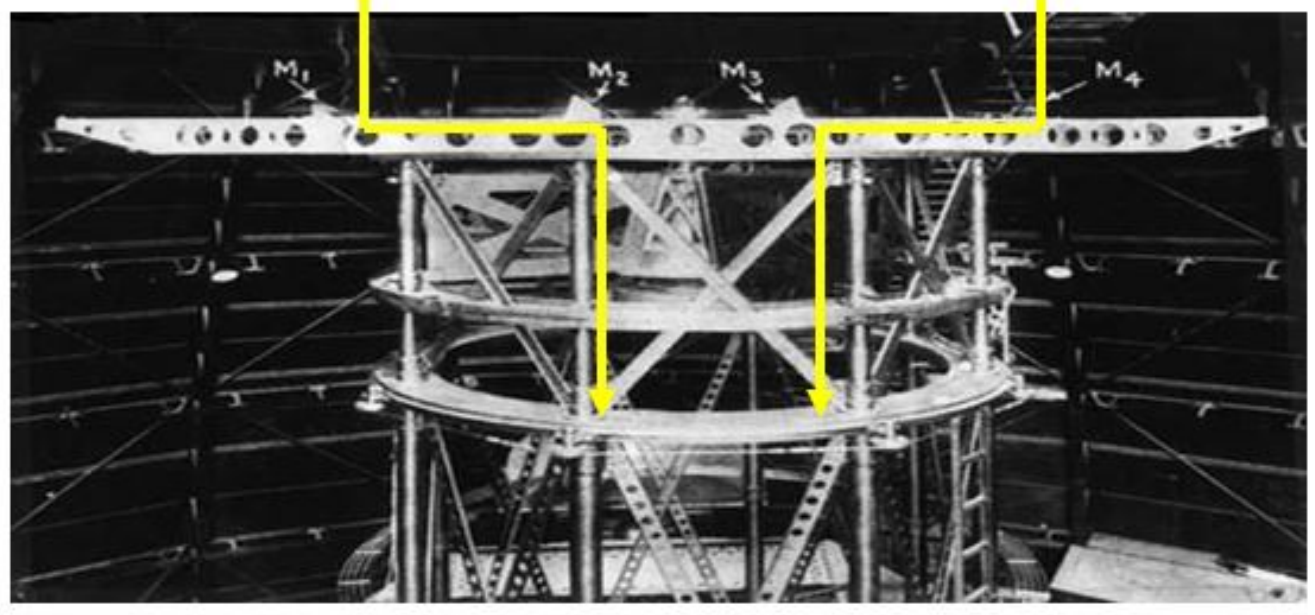

Figure 91. The Michelson interferometer mounted on top of the Hooker 100 inches telescope at Mount Wilson. The light path used 2 flat mirrors in each arm, generating an apparent pupil, as seen from the eyepiece, always the same, independently of the distance of the two entrance mirrors. This fixed output pupil generates a fixed fringe spacing and realizes in fact the first densification. 


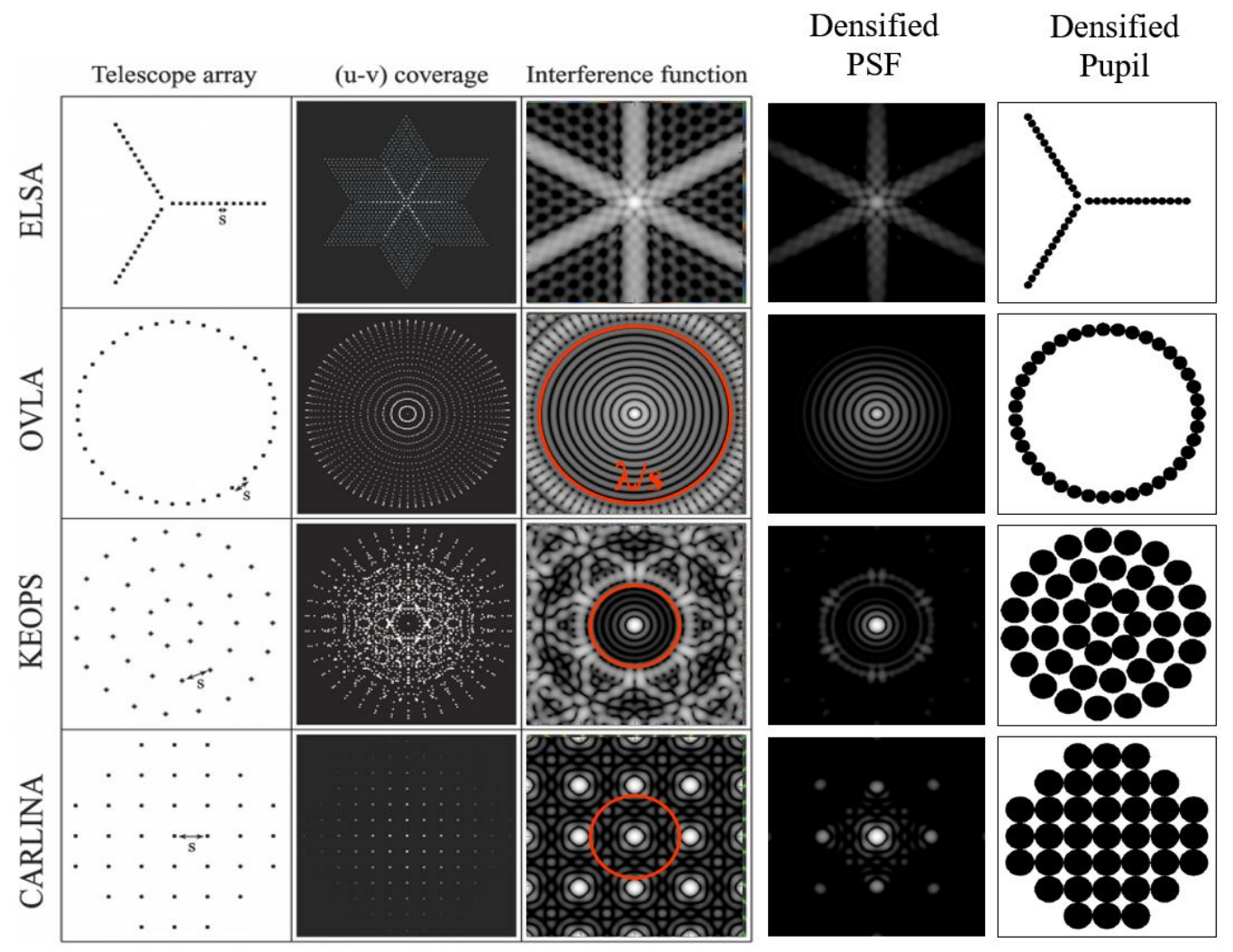

Figure 92. Four different possible interferometric arrays with their respective $(u, v)$ plane coverage, the representation of the interference function, the densified point spread function, and the corresponding densified pupil.

In this section, I have presented the principle of optical interferometry from the direct imaging point of view. With the limitation in the field of view generated by the beam combination and the violation of the strict convolution product in the image-object relationship, we can however consider that an hypertelescope is not different from any imaging telescope. But we must also recognize that it has taken a very long time to obtain images with interferometers and the hypertelescope principle is, today, still in its demonstration phase. The largest part of the astrophysical publications in optical interferometry continue to work with squared visibility, closure phase, triple product amplitude, and model reconstruction or parametric imaging. The main reason behind the current limitations is again a problem of coherence and its strong limitation because of the atmospheric turbulence and the actual implementation of long baseline interferometers. This is also a budgetary issue but we will not cover this aspect of the problem in the present paper.

\section{The reality of the coherence}

If we look again at Eq. 197 in Sec. 2, defining the radius of coherence $r_{c}$, we can easily deduce that for a star with an apparent angular diameter of $10 \mathrm{mas}$, at a wavelength of $1 \mu \mathrm{m}$, the radius of coherence is of the order of $13 \mathrm{~m}$. Considering a spectral bandwidth of $0.1 \mu \mathrm{m}$, 


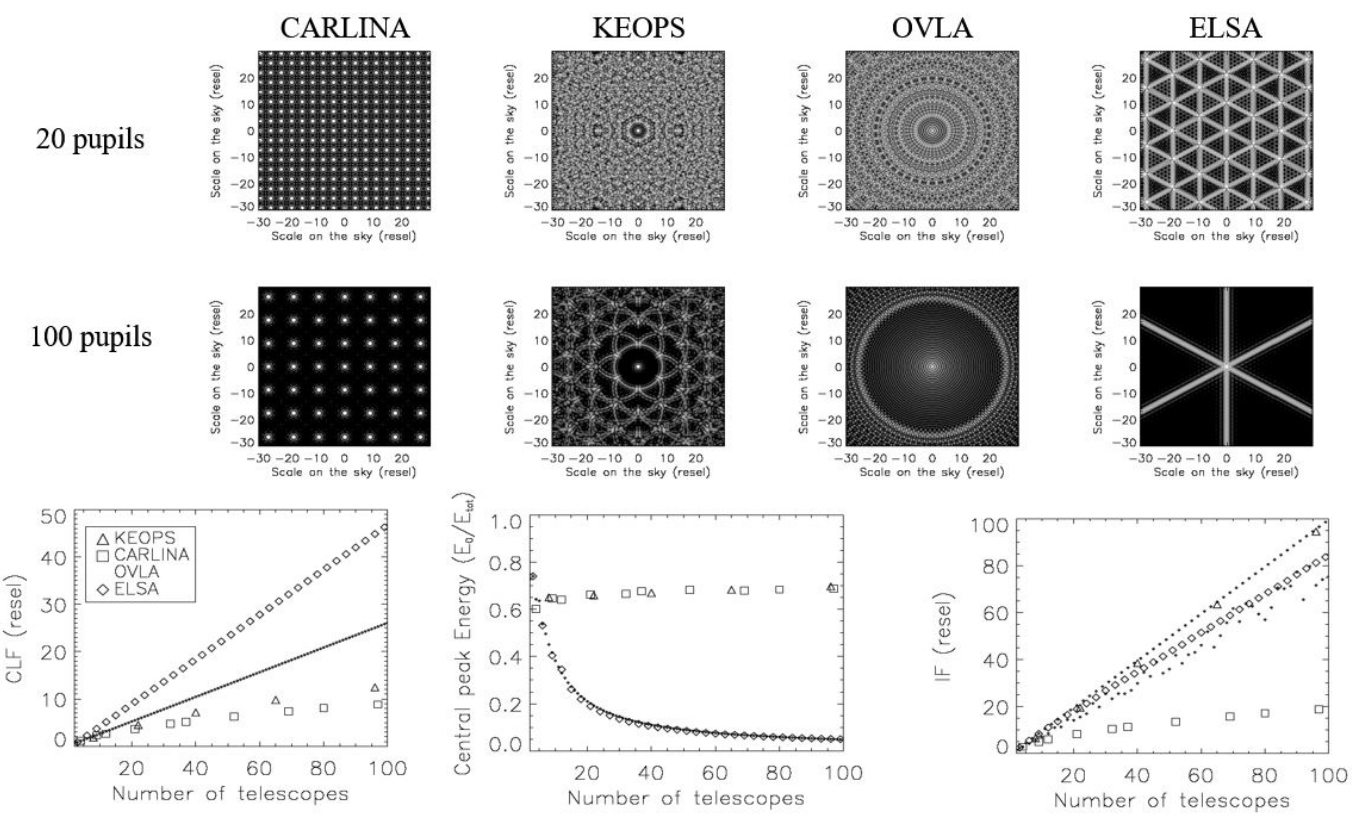

Figure 93. The four interferometric arrays are considered with a variable number of subpupils and the imaging properties are compared, in terms of Clean Field, of encircled energy, and of interferometric field of view.

this corresponds to a coherence duration of $3 \times 10^{-14} s$ and finally a coherence length of $10 \mu m$. It means that, independently of any other consideration, the optical paths between the different arms of the interferometer should be equalized at a level of a fraction of the coherence length if one wants to be able to detect the interferometric pattern and to measure it with good quality. For obvious reasons of correlation, the interferometric signal (the fringe contrast) depends on the residual error on the optical path and if this error is not stable the transfer function of the interferometer is degraded.

Interferometers have been equipped with different kinds of delay lines to compensate for the earth rotation and to guaranty the equalization of the optical paths. The usual setup of such delay lines is a mechanical carriage moving on rails thanks to a motorized wheel. The optical system, usually a cat's eye, is supported by a second carriage, whose position could be corrected precisely with a second level of control loop over a small stroke of one millimeter or less, usually made of a voice-coil. It permits to compensate the defaults of the main driving system. And finally the secondary mirror of the cat's eye is usually supported by a piezo translation stage able to correct the optical path at a very high frequency (a few kiloHertz) over a very small stroke.

The combination of these three control loops permits strokes of a few tens of meters with a precision less than 10 nanometers for speeds up to $10 \mathrm{~mm} / \mathrm{s}$. An example is presented in Fig. 94. Today the technology of delay lines is very mature and the performance is excellent, even at the shortest wavelengths.

There is however a second important difficulty in terms of coherence of the stellar wavefronts due to the atmospheric turbulence. The gradients of temperature and speed inside the turbulent medium generate important variations of the index of refraction of air. When the turbulence is developed, a statistical description of the residual coherence is usually made. 


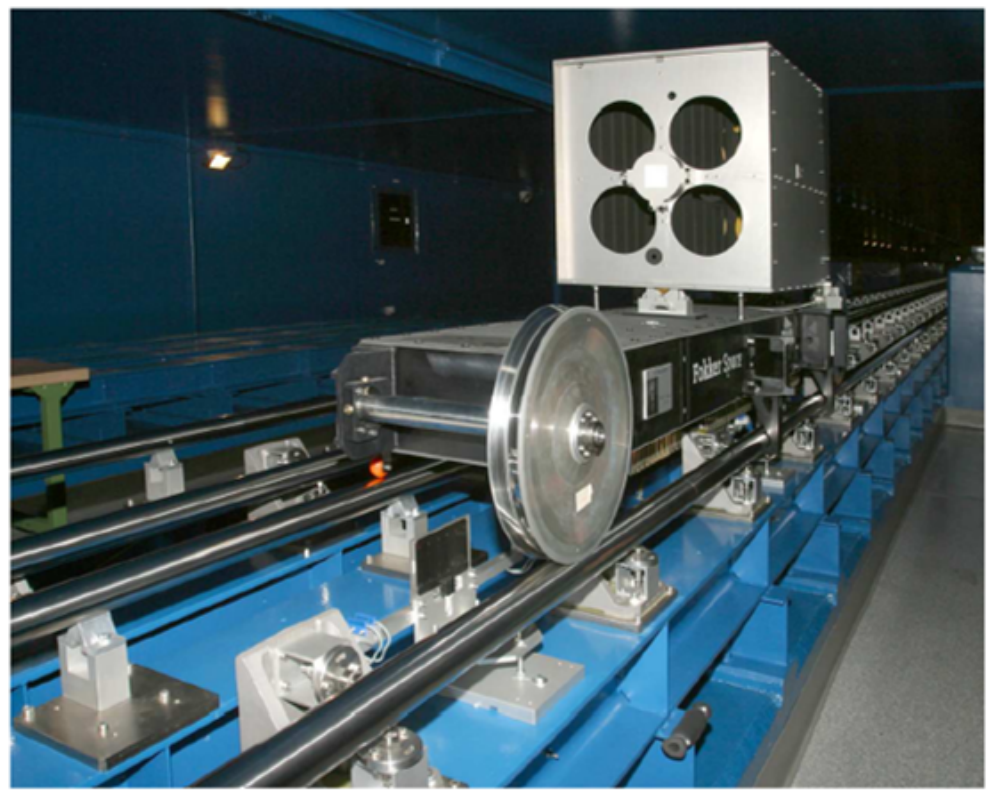

Figure 94. One of the VLTI delay line installed on its rails and supporting the cat's eye.

Astronomical sites are of course carefully studied in terms of seeing properties in complement with overall weather and transparency characteristics. The spatial coherence of the atmosphere is usually described by the Fried parameter $r_{0}$, of the order of $10 \mathrm{~cm}$ in good sites. The temporal coherence is designed by the coherence time $\tau_{0}$, of the order of $10 \mathrm{~ms}$. Finally, at least at visible wavelength, there is an additional characteristic that has to be taken into account which is called the spectral coherence of the atmosphere, of the order of $30 \mathrm{~nm}$ in the $\mathrm{R}$ band. This quantity corresponds to the decorrelation of the speckles and fringes between two distant spectral bands [12]. All these parameters are usually correlated, even if one can find sites with large $r_{0}$ and small $\tau_{0}$ but we consider that the hypervolume of coherence (the one that is directly related to the quality of the interferometric measurement) varies as the power 4 of the turbulence (two spatial dimensions, one temporal, and one spectral). Moreover, the spatial and temporal coherence $\left(r_{0}\right.$ and $\left.\tau_{0}\right)$ vary as $\lambda^{6 / 5}$ which also explains why near and mid infrared interferometry has been preferred for a long time, in addition of course to the simple consideration related to the size of the wavelength.

To avoid the effects of the atmospheric turbulence, one obvious solution is to implement interferometers in space. This has been pushed a lot twenty years ago, especially for the science case of the direct characterization of earth-like planets (TPF, Darwin) but the cost of these missions and, on certain aspects, the technological maturity have killed the efforts. Moreover, the maturity of the ground interferometers was also not well established at that time. Finally, today, the situation of space interferometers is not encouraging at all and no clear path forward could be established.

The progress of technology and of fast control loops on ground systems (see the paper by F. Martinache in this book) have permitted to develop cophasing devices permitting to compensate the negative aspects of the atmospheric turbulence. The individual telescopes could be equipped with adaptive optics (usually simple one based on the principle of Single Conjugated Adaptive Optics for a very narrow field). Coherencing devices have been developed 
for a long time [13] in order to stabilize the optical path difference within a small fraction of the coherence length. More recently, and after the pioneering work of FINITO [14] on the VLTI, cophasing devices permitting to stabilize the optical path difference within a fraction of the wavelength, have been successfully implemented within the GRAVITY instrument on the VLTI [15].

So, the coherence of the stellar wavefront could be measured with the current interferometers implemented on ground if different levels of active and adaptive compensation are implemented: delay lines for the compensation of the earth rotation, adaptive optics on each individual telescope to correctly use the full collecting area, and finally a cophasing device to correct for the difference of piston between the different arms of the interferometers. With the effective implementation of all these active systems, the different samples of the incoming stellar flat wavefront are fully recophased and the ultimate performance could be reached, almost like in a classical giant telescope with a diluted pupil.

\section{Practical considerations for the implementation of an interferometer}

Looking back now to the schematic representation of the two types of interferometers (see Fig. 89), it is clearly understandable that the Fizeau configuration (A) is not easy to implement on ground (because of the need for a giant driving system) and the classical way of doing interferometers on ground is the second scheme (case B), usually called the Michelson configuration. In this case the two segments of the giant parabola are replaced by independent telescopes and the light is recombined at the common focus.

We should focus first our attention on one general geometric particularity of this system. The entrance pupil plane is now made of independent subpupils that are separated by tens or hundreds of meter. Therefore the quality of the optical transfer function will highly depend on the control of the sub pupils, in terms of transverse and longitudinal position. Moreover the global pupil plane is no longer perpendicular to the direction of pointing, as it was the case in the Fizeau configuration or for any standard telescope. Optical delay appears between the two arms and delay lines are mandatory. An important atmospheric dispersion may appear because of the prism of air above the pupil, and finally the subpupils are at variable distance from the focus which may generate Fresnel diffraction [16]. The first conclusion is that a complex optical system is needed between the collection of the wavefronts and the interferometric focus (see Fig. 95).

Now that the beams are correctly transported to the common interferometric focus we should optically form the interferometric signal described in Eq. 201 of Sec. 2. Two options could be considered: a coaxial beam combiner with a temporal modulation (see left panel of Fig. 96) or a multi-axial combiner with a spatial modulation (right panel of Fig. 96). The first mode is well adapted to single pixel detectors and was, therefore, used for a long time at the beginning of infrared interferometry. The low noise and large number of pixels of the detectors in the visible have permitted to prefer the second scheme in the visible. The recent progresses of infrared detectors have progressively reduced the interest of the co-axial combination.

With these schemes of combination, the interferometric equation (Eq. 203 of Sec. 2) is formed with a dependence of the term $\phi$ in the cosinus function, either with time (co-axial scheme) or position (multi-axial scheme).

Pending the actual implementation of direct imaging interferometers, the current instruments are based on the measurements of the fringe properties, mainly contrast and phase. The first measurement is the contrast $\mathrm{C}$ of the fringes which is usually defined as: 

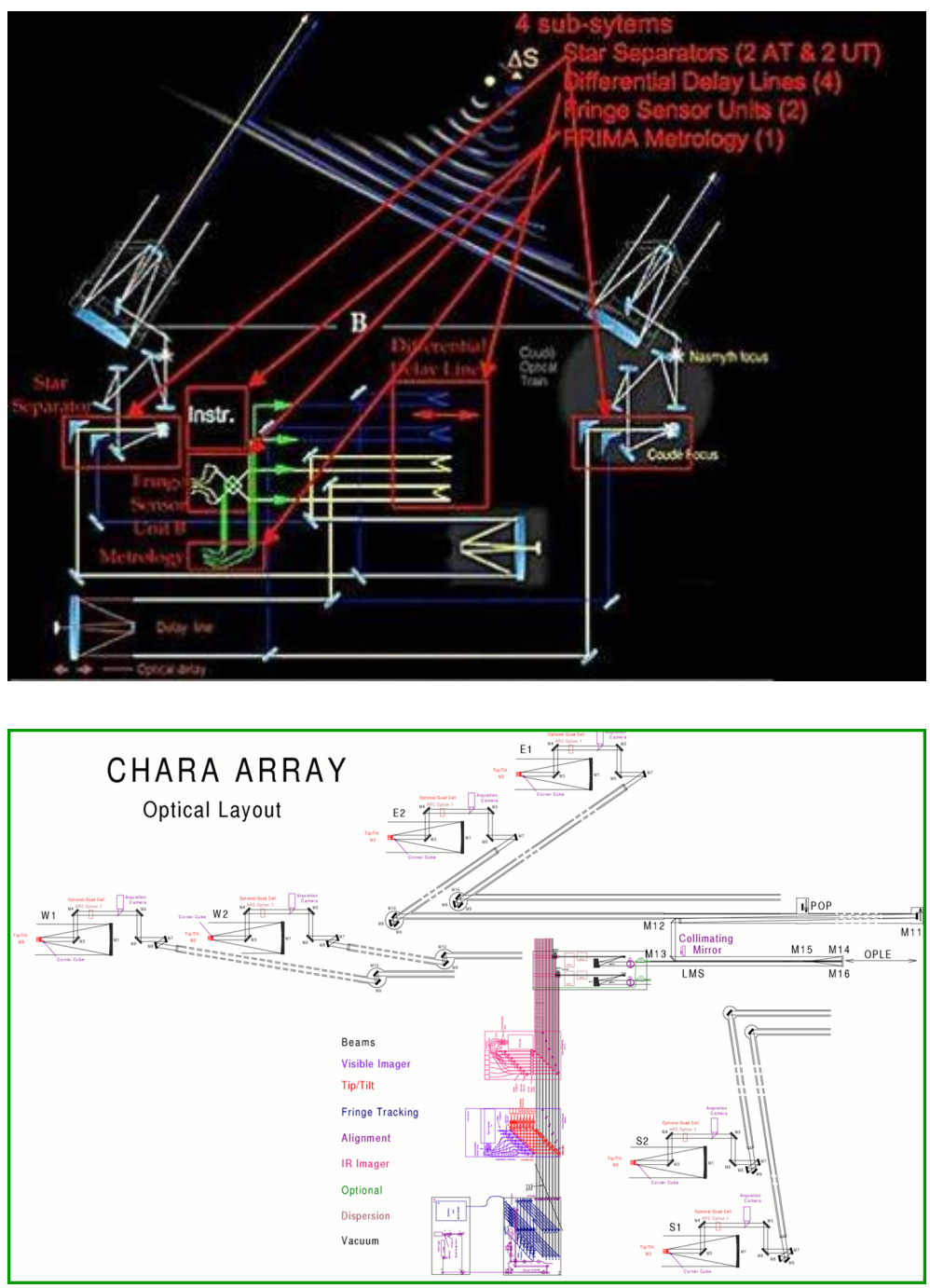

Figure 95. The complex optical Coude train of the VLTI (top) and CHARA (bottom) interferometers.

$$
C=\frac{I_{\max }-I_{\min }}{I_{\max }+I_{\min }}
$$

With the simplifying hypothesis $I_{2}=I_{1}$ meaning that no photometric difference exists in the two beams, we can easily deduce that the contrast is an estimation of the complex degree of mutual coherence, also called the squared visibility $V^{2}$.

$$
C=V^{2}=\frac{\left|\psi_{1} \psi_{2}{ }^{*}\right|}{\sqrt{\left|\psi_{1}\right|^{2}\left|\psi_{2}\right|^{2}}} .
$$

It can be shown also that extracting the squared visibility could be done by the so-called $\mathrm{ABCD}$ technique. The principle is to measure the intensity of the signal over four phase 
Coaxial

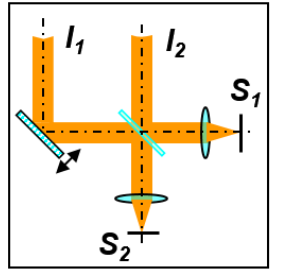

Temporal sampling

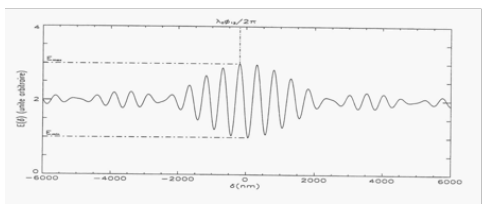

Multi-axial

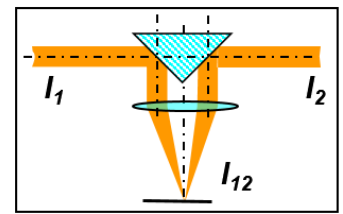

Spatial sampling

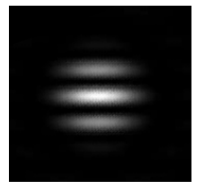

Figure 96. The two generic beam combiners for optical interferometry: (left) co-axial and temporal modulation, (right) multi-axial and spatial modulation. The bottom raw gives a representation of the fringe signal, as a function of time (left) or as a function of the position on the detector (right).

intervals covering: $A=[0 ; \pi / 2], B=[\pi / 2 ; \pi], C=[\pi, 3 \pi / 2]$, and $D=[3 \pi / 2,2 \pi]$. The contrast is obtained with the following formula:

$$
C=V^{2}=\frac{\pi}{\sqrt{2}} \frac{\sqrt{\left(I_{A}-I_{C}\right)^{2}+\left(I_{B}-I_{D}\right)^{2}}}{I_{A}+I_{B}+I_{C}+I_{D}}
$$

The $\mathrm{ABCD}$ technique is more usually used for measuring the phase of the fringes on a very efficient way and the first technique with $I_{\min }$ and $I_{\max }$ is not easily implementable because of the fast motion of the fringes due to the atmospheric turbulence. Mathematically speaking, these two techniques are in fact equivalent, from a numerical point of view, to discrete Fourier transform of the fringe equation. And this is in fact the usual way to measure the fringe contrast, through a Fourier transform of the raw images.

The measurement of the phase of the fringes is usually more complicated because of the atmospheric turbulence. Differential measurements between two different spectral bands are possible and permit to get rid of the atmospheric phase. This has been used a lot and it is usually estimated through the argument of the cross-spectrum:

$$
\operatorname{Arg}\left(<\tilde{I}_{\lambda_{1}} \tilde{I}_{\lambda_{2}}^{*}>\right)=\operatorname{Arg}\left(\tilde{O}_{\lambda_{1}}\left(\frac{B}{\lambda}\right)\right)-\operatorname{Arg}\left(\tilde{O}_{\lambda_{2}}\left(\frac{B}{\lambda}\right)\right) .
$$

Another possibility, inspired from what has been developed for a long time in radioastronomy [17], is the so-called closure phase, as mentioned in Section 3 and Fig. 4. One has to consider a triplet of telescopes as in Fig. 6:

The phases $\left(\psi_{12}, \psi_{23}\right.$, and $\left.\psi_{31}\right)$ of the fringes over the different baselines could be expressed as following:

$$
\begin{aligned}
& \psi_{12}=\Phi_{12}+\varphi_{1}-\varphi_{2} \\
& \psi_{23}=\Phi_{23}+\varphi_{2}-\varphi_{3} \\
& \psi_{31}=\Phi_{31}+\varphi_{3}-\varphi_{1}
\end{aligned}
$$




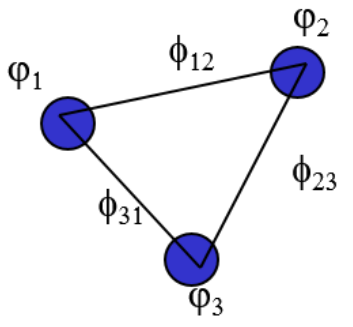

Figure 97. A triplet of telescopes used for measuring closure phase. The atmospheric phases on each telescope are indicated $\left(\varphi_{1}, \varphi_{2}\right.$, and $\left.\varphi_{3}\right)$. Over the three baselines (12-23-31), the phases $\left(\Phi_{12}, \Phi_{23}\right.$, and $\left.\Phi_{31}\right)$ of the Fourier Transform of the object brightness distribution are also indicated.

Getting rid of the atmospheric phase is possible by forming the following expression called the closure phase:

$$
\text { ClosurePhase }=\psi_{12}+\psi_{23}+\psi_{31}=\Phi_{12}+\Phi_{23}+\Phi_{31} .
$$

Summing the phase of the three fringes system on a closed triplet of telescopes permits to obtain an information on the phase of the Object without the perturbation of the atmospheric phase.

With the squared visibility, the differential phase and differential visibility, and the closure phase, we have now a complete description of the interferometric observables. We have seen at the end of Sect. 3, and in particular, in Fig. 88, how many of these quantities are accessible for an interferometer with a number $\mathrm{N}$ of telescopes. In the next Section, I will present a short overview of the existing facilities and of their main science drivers.

\section{A modern panorama of optical long baseline interferometry}

After a few decades of development, modern optical interferometry is now focused on four facilities: ESO/VLTI, CHARA, NPOI, and MROI.

\subsection{NPOI}

$\mathrm{NPOI}^{2}$ is a collaboration between US Naval Observatory (USNO), Naval Research Lab (NRL), and Lowell Observatory. NPOI has a long story and was part of the pioneering installations at the end of the previous century. The major part of its program is dedicated to global precision astrometry for the maintenance of the coordinates and proper motion of reference stars. But NPOI has also obtained very impressive results in stellar physics, on single stars or multiple systems. It is equipped with a Classic combiner based on a temporally modulating combiner and avalanche photodiodes. It covers the visible domain (550 to $850 \mathrm{~nm}$ ) with a resolution of 40 and offers 16 independent spectral channels. NPOI has a sensitivity limit of $m_{V} \simeq 5.5$ with the $6+4$ (imaging+astrometric) siderostats, $12 \mathrm{~cm}$ in diameter, equipping the array. Recently a new EMCCD-based spatially modulating combiner called VISION has been developed [18]. It can accommodate up to 6 beams and offers two spectral resolutions (200 and 1000) in the visible domain. NPOI is currently being upgraded with larger telescopes ( $1 \mathrm{~m}$ in diameter) and the infrastructure for enabling the $450 \mathrm{~m}$ baselines of the Anderson Mesa site.

\footnotetext{
${ }^{2}$ http://www2.lowell.edu/rsch/npoi/index.php
} 


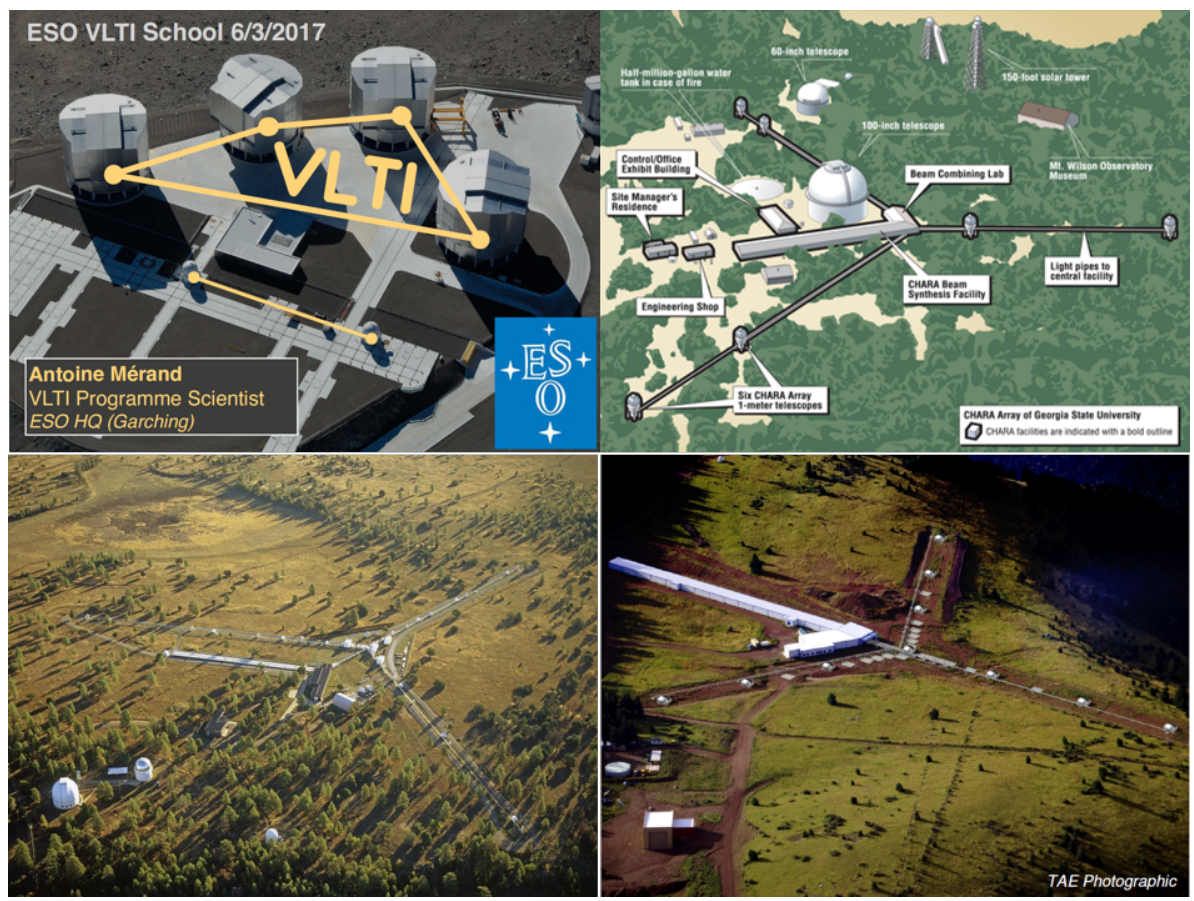

Figure 98. A panorama of the four optical interferometers existing today in the world. We recognize the Very Large Telescope Interferometer or VLTI (upper-left panel) installed at Cerro Paranal on the premises of ESO. The Center for High Angular Resolution in Astronomy or CHARA Array, developed by the Georgia State University (upper-right panel) is installed on Mount Wilson, California. In the bottom raw, the Navy Precision Optical Interferometer (NPOI) is located on Mesa Anderson, close to Flagstaff - Arizona (bottom-left panel). And finally the Magdalena Ridge Optical Interferometer (MROI) is today in construction phase on the site of the Magdalena Ridge Observatory - New Mexico (bottom-right panel).

\subsection{MROI}

The $\mathrm{MROI}^{3}$ is in construction phase. The baseline plan is to equip the Array in Y-shape with ten $1.4 \mathrm{~m}$ telescopes with the possibility of relocation on baselines between 8 and $350 \mathrm{~m}$. It is designed for imaging the central zone of active galactic nuclei, the study of the star and planet formation, and the physical processes of stellar accretion and mass loss. The first telescope has been delivered on site during Spring 2018 and many developments are still ongoing like innovative evacuated delay lines or a fringe tracker for phase stabilization. The implementation of the full facility is however funding dependent.

\subsection{The VLTI array}

The ESO/VLTI Array ${ }^{4}$ is installed on the ESO premises at Cerro Paranal in Chile. The four 8.2-m Unit Telescopes (UTs) and the four 1.8-m Auxiliary Telescopes (ATs) are the light collecting elements of the VLTI. The UTs are set on fixed locations while the ATs can be

\footnotetext{
${ }^{3}$ http://www.mro.nmt.edu/about-mro/interferometer-mroi/

${ }^{4}$ https://www.eso.org/sci/facilities/paranal/telescopes/vlti.html
} 
relocated on 30 different stations. After the light beams have passed through a complex system of mirrors and the light paths have been equalized by the delay line system, the light re-combination is performed for quadruplet of telescopes with the beam combiners PIONIER, GRAVITY, and MATISSE. The first generation instruments (VINCI, MIDI, AMBER) are now decommissioned. Due to its characteristics, the VLTI has become a very attractive means for scientific research on various objects like young pre-main sequence stars and their protoplanetary disks, post-main sequence mass-losing stars, binary objects and their orbits, solar system asteroids, our galactic center, and extragalactic objects such as active galactic nuclei.

PIONIER [19] is a H-band 4-beam combiner instrument. It was originally a visitor instrument that by agreement with IPAG is offered to the ESO community from P96 onwards as a facility instrument, i.e. without the need for collaborating with the instrument team, and is offered both in visitor and service mode for normal, large, and monitoring programs. PIONIER combines either beams from the 8.2-m Unit Telescopes or from the 1.8-m Auxiliary Telescopes to provide visibilities of six different baselines, as well as four closure phase measurements, simultaneously. PIONIER features low resolution spectroscopic optics to measure at six different wavelengths within the H-band, increasing the $(\mathrm{u}, \mathrm{v})$ coverage, or can work in integrated light for sensitivity enhancement on faint targets.

GRAVITY [15] is an interferometric instrument operating in the $\mathrm{K}$ band, between 2.0 and $2.4 \mu \mathrm{m}$. It combines 4 telescope beams and is designed to perform both interferometric imaging and astrometry by phase referencing. The instrument delivers spectrally dispersed interferometric quantities in low, medium, and high spectral resolution. GRAVITY's angular resolution is set by the maximum separation of the employed telescopes and the field of view of the instrument. In imaging mode, GRAVITY is able to resolve features between 4 mas (milli-arcsecond) and 50 mas with the UTs, and between 2 mas and 140 mas with the ATs. The astrometric accuracy is also given by the maximum separation of the telescopes and will be of the order of few 10 to $100 \mu$ as. GRAVITY is equipped with a dedicated fringe tracking subsystem and with a dedicated IR wavefront sensing system (CIAO) on the UTs.

MATISSE [20] is a mid-infrared spectro-interferometer combining the beams of up to $4 \mathrm{UTs} / \mathrm{ATs}$. The instrument sensitivity, sampling and throughput are optimized for L and N. The $\mathrm{L}$ band is specified from 3.2 to $3.9 \mu \mathrm{m}$ and the $\mathrm{N}$ band from 8.0 to $13.0 \mu \mathrm{m}$. MATISSE will operate also in $\mathrm{M}$ band, from 4.5 to $5.0 \mu \mathrm{m}$. The $\mathrm{L}, \mathrm{M}$ and $\mathrm{N}$ bands can be observed simultaneously. The instrument will be able to observe with different spectral resolutions. Two spectral resolutions are possible in $\mathrm{N}$ band $(\mathrm{R} \simeq 30, \mathrm{R} \simeq 200)$ and 3 in $\mathrm{L} \& \mathrm{M}$ bands $(\mathrm{R} \simeq$ $30, \mathrm{R} \simeq 500$ for $\mathrm{L}$ and $\mathrm{M}, \mathrm{R} \simeq 1000$ for $\mathrm{L}$ only). Due to readout time, the full simultaneous coverage of the L\&M bands in low and medium resolutions, and the full coverage of the $\mathrm{L}$ band in high spectral resolution require an external fringe tracker. MATISSE will measure: coherent flux, visibilities, closure phases and differential phases. Differential visibilities can also be derived. These quantities will be measured as a function of the wavelength in the selected spectral bands and resolutions. The objective of MATISSE is image reconstruction. It will extend the astrophysical potential of the VLTI by overcoming the ambiguities existing in the interpretation of simple visibility measurements.

\subsection{The CHARA array}

The Center for High Angular Resolution Astronomy $\left(\mathrm{CHARA}^{5}\right)$ optical interferometric array of six telescopes is located on Mount Wilson, California. Each telescope of the CHARA Array has a light-collecting mirror 1-meter in diameter. The telescopes are dispersed over

\footnotetext{
${ }^{5}$ http://www.chara.gsu.edu/
} 
the mountain to provide a two-dimensional layout that provides the resolving capability of a single telescope with a diameter of 330 meters. Light from the individual telescopes is transported through vacuum tubes to a central Beam Synthesis Facility in which the six beams are combined together. When the paths of the individual beams are matched to an accuracy of less than one micron, after the light traverses distances of hundreds of meters, the Array then acts like a single coherent telescope for the purposes of achieving exceptionally high angular resolution. The Array is capable of resolving details as small as 200 micro-arcseconds. The strength of the CHARA Array besides in the number and size of its individual telescopes, its ability to operate at visible and near infrared wavelengths, and its longest baselines of 330 meters.

The CLASSIC and CLIMB beam combiners [21] of the CHARA Array are open air, aperture plane, broadband, single spectral channel instruments optimized for sensitivity. CLASSIC is the original two beam combiner used for the first science at CHARA, and it still has the faintest magnitude limit. CLIMB is a three beam expansion of CLASSIC that also provides closure phase measurements. It is a very useful coherence tracker for the visible combiners.

JouFLU [22] is a two telescope beam combiner operating in the near infrared $\mathrm{K}$ band $(\lambda=$ $2.20 \mu \mathrm{m})$. JouFLU spatially filters the light through optical fibers to produce high precision visibility measurements.

The Michigan Infrared Combiner (MIRC [23]) combines the light from all six CHARA telescopes simultaneously. It disperses the light across 8 spectral channels in the near infrared H-band $(1.6 \mu \mathrm{m})$. MIRC can be used to create images of stellar surfaces. The precision closure phases are well suited to detecting faint binary companions.

PAVO [24] is an integral-field-unit for measuring spatially-modulated fringes in the pupil plane. It spectrally disperses light over a 630-950 nm bandwidth $(\mathrm{R}=30)$. Currently, PAVO can be used to combine the light from two telescopes.

The Visible spEctroGraph and polArimeter (VEGA [25, 26]) was installed in September 2007 at the CHARA array. VEGA measures spectrally dispersed interference fringes, providing a spatial resolution of 0.3 mas and spectral resolutions from $\mathrm{R}=6,000$ to 30,000 over a wavelength of 480-850nm. It can combine 2,3 , or 4 telescopes.

\section{The study of stellar surface and their close environment using interferometry}

In this section we will present some of the most exciting results obtained using interferometry in the recent years. We will focus on stellar physics, i.e. the study of stellar surface and their close environment, following the framework of the 2017 edition of the Evry Schatzman school of the French national program of stellar Physics (PNPS). One can see in Fig. 99 that the number of refereed publications using interferometry (considering all topics) has grown continuously to a maximum in 2009 , and remains a very active domain of research. The new generation of instruments that are just arriving now will probably continue this growth.

As we will show, interferometric, direct and Doppler imaging are very complementary approaches, while the synergy between interferometry and asteroseismology is powerful in the context of Gaia and PLATO space missions.

\subsection{Imaging at very high angular resolution}

Four centuries after Giordano Bruno, one of the dreams in astronomy is to make the image of an exo-Earth located at say 10 light years to find traces of life. However, to achieve this objective, it would be necessary, for example, to build a $150 \mathrm{~km}$ space hypertelescope (see 


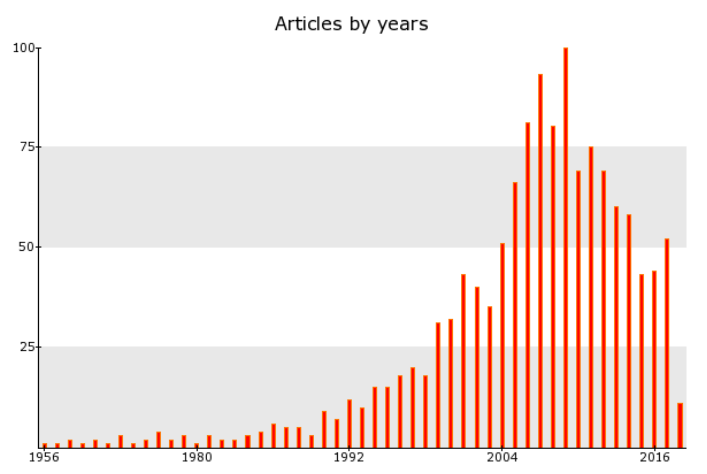

Figure 99. Histogram of refereed publications in interferometry (considering all topics). Source: statistics done by the Jean-Marie Mariotti Center (JMMC, http://apps.jmmc.fr/bibdb/).

Fig. 100). This example shows the difficulty of the task, with both problems of angular resolution and contrast. Thus, what can be done first, and is in fact absolutely necessary to understand the planet, is to image the stars and in particular, the most resolved stars, such as giant stars or red supergiants.

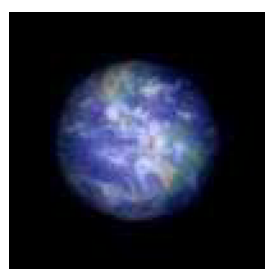

Figure 100. Simulated image of the planet Earth located 10 light-years away and observed by a 150 $\mathrm{km}$ hypertelescope consisting of a network of $1503 \mathrm{~m}$ mirrors in the visible domain [27].

\subsubsection{Giant and red supergiants stars}

We can for instance cite the interferometric image of the red giant $\pi^{1}$ Gruis (radius of 350 $\mathrm{R}_{\odot}$ ), obtained by the PIONIER instrument at VLTI, which reveals for the first time granulation patterns on the surface of a star [28] (see Fig. 101). Valuable information on the geometry and dynamics of the granulation is obtained from interferometric observations, even if the coverage of the spatial frequency plane is insufficient to produce an image. Several studies have been conducted, first on the red supergiant Betelgeuse [29], then Antares [30] and finally on the red giant $\beta$ Oph with the instrument MIRC on the interferometer CHARA [31]. Let's also mention a series of remarkable images obtained on a giant, $\zeta$ And, with the instrument MIRC on CHARA [32] (see Fig. 102) which made it possible to detect a dark spot during a cycle of rotation of the star. Note that these observations of red giants or supergiants stars generally require a heavy investment in terms of numerical computation time in order to produce a 3-dimensional model of the star [33] to interpret the visibility and phase closure measurements. 


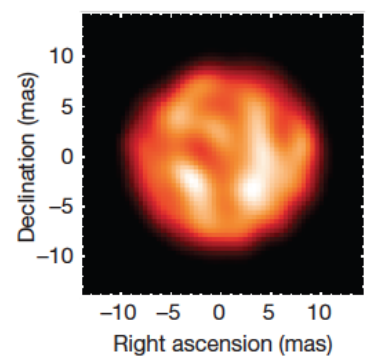

Figure 101. The surface of the red giant $\pi^{1}$ Gruis imaged by the PIONIER instrument on the VLT [28], i.e. with a hectometric base and in $\mathrm{K}$ band.

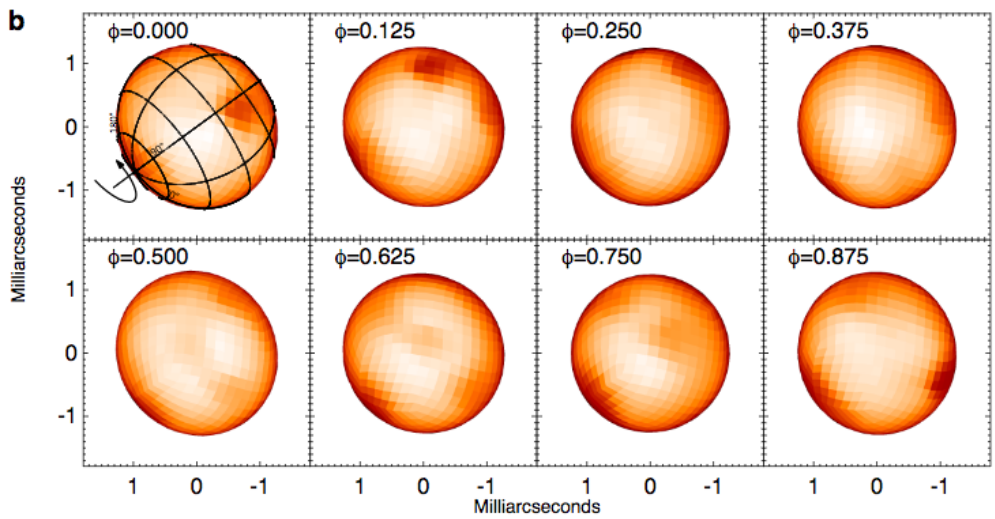

Figure 102. The surface of the giant $\zeta$ And obtained with the instrument MIRC on CHARA [32]. We can see a persistent spot at the pole of the star and a transient spot moving with the rotation of the star.

To go further in the analysis of this type of astrophysical objects, it becomes interesting to adopt precisely a multi-technique or multi-wavelength strategy. We will give two examples here.

- First, it should be noted that a strong linear polarization was detected in the Betelgeuse [34] atomic lines, in several other red supergiants [35], as well as in Mira or RV Tau pulsating stars [36]. This polarization in the spectral lines makes it possible to go back to the geometry of a possible bright spot on the disk of the star, a spot that can also be detected by interferometry. Thus, the bright spots observed on the interferometric images of Betelgeuse [29] have also been detected by spectro-polarimetry [34]. Thus, a remarkable synergy between interferometric and Doppler imaging appears. And it may be noted in this regard that the VEGA instrument on the CHARA interferometer has a polarimetric mode, thus allowing spectro-polaro-interferometry, but this mode is not used on a regular basis [37].

- Another example is given by a multi-wavelength AGB L2 Pup approach, observed in the visible with the ZIMPOL/SPHERE instrument of the VLT [38] on the one hand, and with the radio interferometer ALMA [39] on the other hand. What is remarkable here is that the angular resolution of a telescope of 8 meters in the visible (16 mas) is almost identical to 
that obtained by the kilometer bases of ALMA in the radio domain at $0.9 \mathrm{~mm}$ (14 mas). It thus becomes possible to superimpose two images at identical angular resolutions, but at two different wavelengths. While SPHERE gives indications on the geometry of the observed object, ALMA provides, because of its spectral resolution, constraints on the kinematics of the environment in the CO line (see Fig. 103). It is interesting to mention that we will obtain in the future the same type of synergy, i.e. images of equivalent angular resolution, between ZIMPOL/VLT in the visible and a K-band ELT, or between an ELT in the visible and MATISSE/VLTI in N-band (Fig. 104).

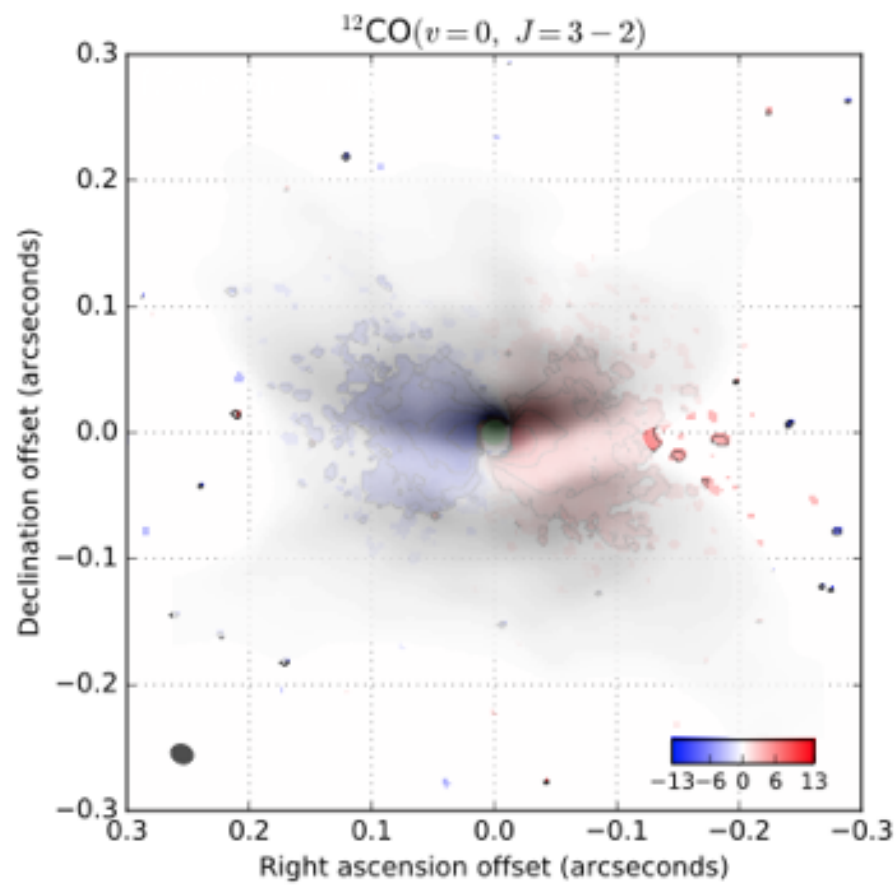

Figure 103. Superimposition of L2 Pup images obtained with ZIMPOL/SPHERE [38] and ALMA [39]. The image in gray color corresponds to the observations of ZIMPOL/SPHERE instrument on the VLTI. In color (blue / red) appears the velocity fields (in $\mathrm{km} . \mathrm{s}^{-1}$ ) as measured by ALMA. These two images have the same angular resolution, which perfectly illustrates the potential of a multi-wavelength approach.

Giant and supergiant imaging is thus a very dynamic field of research. But, failing to be able to image the exoplanets, another synergy appears around the detection of the exo-planets by imagery.

\subsubsection{The star/planet interaction}

The high angular resolution is a powerful tool that currently allows, especially with the SPHERE instrument on the VLT to detect exoplanets by imaging. An interesting example concerns the GJ504 system [40]. As the subtitle of this publication "Combining interferometric, radial velocity, and high contrast imaging data" indicates, this is a good example of synergy between IRDIS/SPHERE observations on the VLT which allow to detect the exoplanet, 


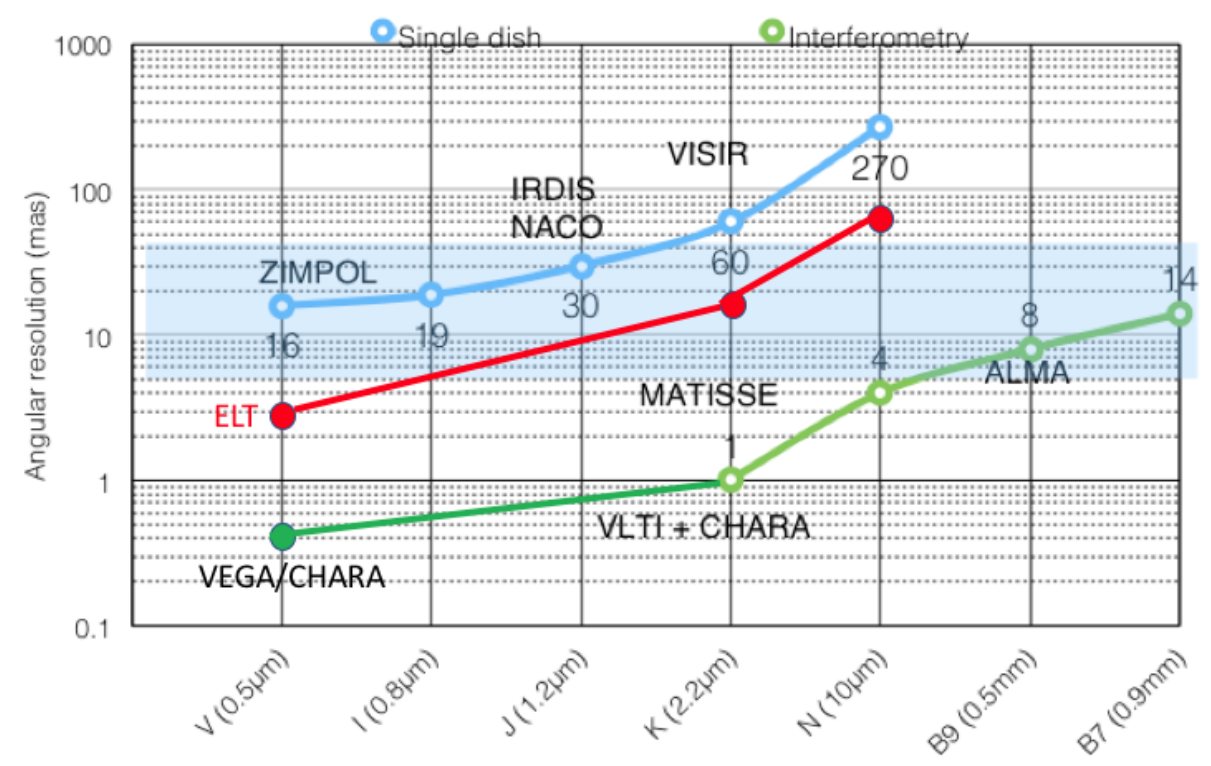

Figure 104. Comparison of the angular resolution of monolithic telescopes and existing interferometric arrays as a function of wavelength. It is thus possible to obtain images of equivalent angular resolution with ZIMPOL/SPHERE on the VLT in the visible and the interferometric ALMA interferometric array in the B9 band at $0.9 \mathrm{~mm}$ (see Fig. 103). In the same way, we can anticipate interesting future synergies between the ELT in the K band, ZIMPOL and ALMA (B9); or between the visible ELT and the MATISSE interferometric instrument on the N-band VLTI.

and interferometric observations VEGA/CHARA [41], which, in turn, make it possible to constrain the angular diameter of the central star. This angular diameter, combined with a measurement of parallax provides the radius of the star, which in turn provide strong constraints on the age of the system, a crucial information to better characterize the fundamental parameters of the star-planet pair.

\subsubsection{The rotating stars}

Fast rotators are generally smaller angularly, and therefore more difficult to image than the giant or supergiant stars, but significant efforts have been made in recent years in this field of research. We can mention for instance the image of the star Achernar, the closest and brightest rotating star of the sky, obtained with the instrument PIONIER on the VLTI. The ' $\mathrm{e}$ ' indicates that there is a Balmer emission line due to the circumstellar environment of the star, which appears and disappears periodically. Achernar's 2014 images show an excess of continuous emission in the $\mathrm{H}$ band with a spatial extension of typically 2 stellar radii [42] (see Fig. 105).

The study of Be's disks is also an active field of research and we can present here the remarkable results obtained on $\phi$ Per with the complementary contribution of the MIRC and VEGA instruments on CHARA. The MIRC instrument (in $\mathrm{H}$ band, with a resolution 4 times lower than in the visible range with VEGA) made it possible to image the disc of the star, as well as the orbit of the companion (Fig. 106). The VEGA data, with the combination of the spatial and spectral resolution of the instrument, provide images of the disk of the star 


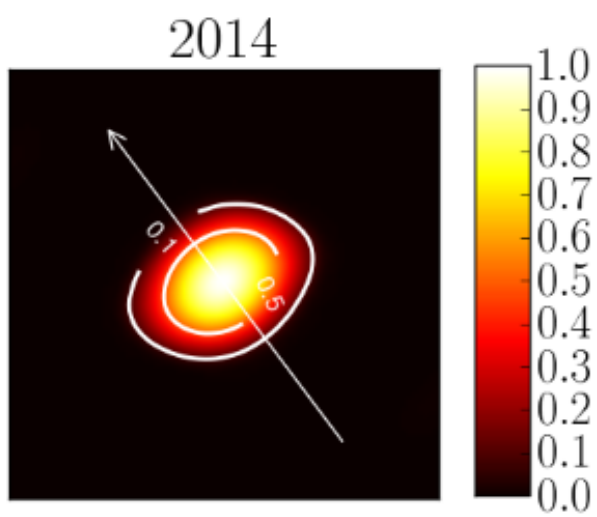

Figure 105. Pionier/VLTI image of Archenar secured in 2014. The arrow corresponds to the axis of rotation of the star whose equatorial angular diameter is close to 2 mas [42]

at different wavelengths in the hydrogen line $\mathrm{H} \alpha$, which gives access to the disc kinematics [43]. This example shows the need of combining interferometric instruments at different wavelengths, and, as such, the availability of the different instruments within CHARA is particularly profitable for many projects in stellar physics. This also applies to the VLTI, and even between the VLTI and CHARA for some objects close to the ecliptic.

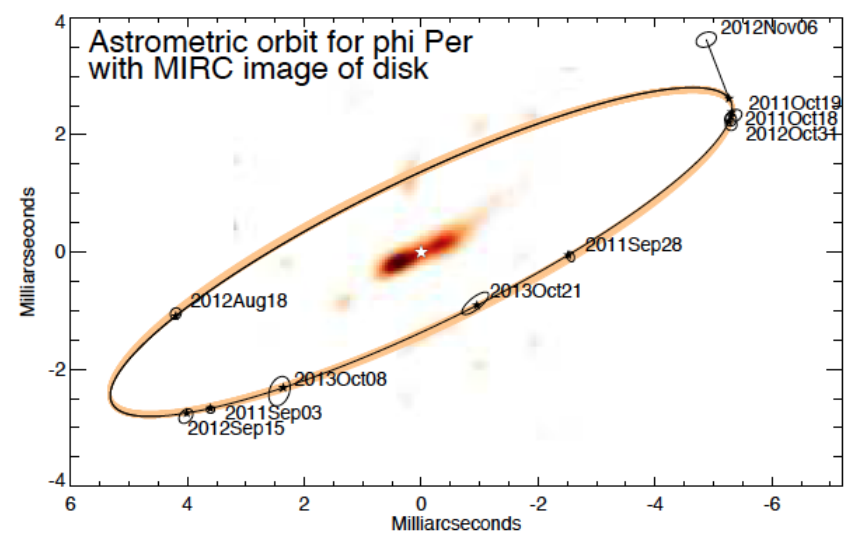

Figure 106. On this image obtained with the instrument MIRC on CHARA, we see the disc of the Be $\operatorname{star} \phi$ Per (the star itself has been removed) as well as the orbit of the companion.

In addition to the environment (disk) of Be-type stars, the surface of rapidly rotating stars is also interesting to study, since it exhibits a flattening effect, as well as a gravitational darkening due to a gradient of temperature and gravity. Interferometry is a technique adapted to the study of these geometric properties. Visibility and closure phase measurements are indeed used to constrain the parameters of the model of the rotating star. For instance, the CHARRON code was recently used to analyze the PIONIER data of Archenar [44]. Beside, it should be mentioned that the gravitational darkening is particularly well contrasted in the visible range, and that the first measurements in this wavelength domain although incomplete 


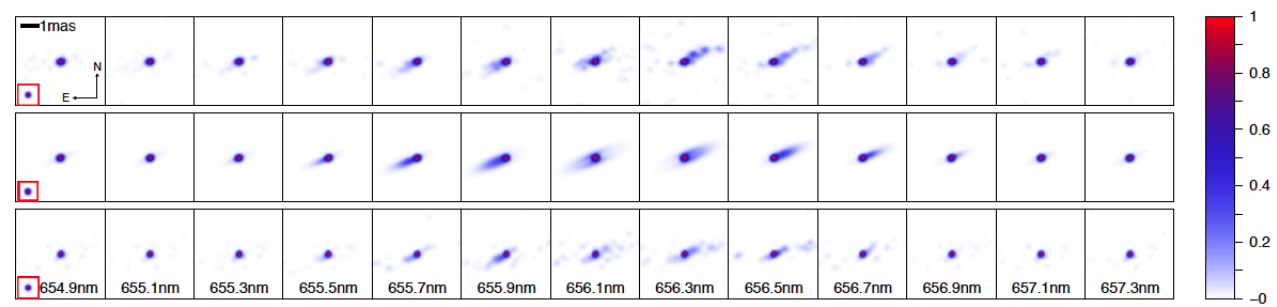

Figure 107. The polychromatic images of $\phi$ Per in the $\mathrm{H} \alpha$ line, a good tracer of the kinematics of the rotating disc) obtained by the VEGA / CHARA instrument (at the top) are compared to a model (medium), itself artificially degraded by a model of the instrument (below). This figure is taken from [43].

(without phase closure) remain valuable. Thus, [45] measured a flattening of $12 \%$ for $\delta$ Per in the visible domain, and deduced a speed of rotation of $57 \%$ of the critical speed (i.e. maximum speed allowed for the stability of the star) .

\subsection{Cepheids and eclipsing binaries as distance indicators in the universe}

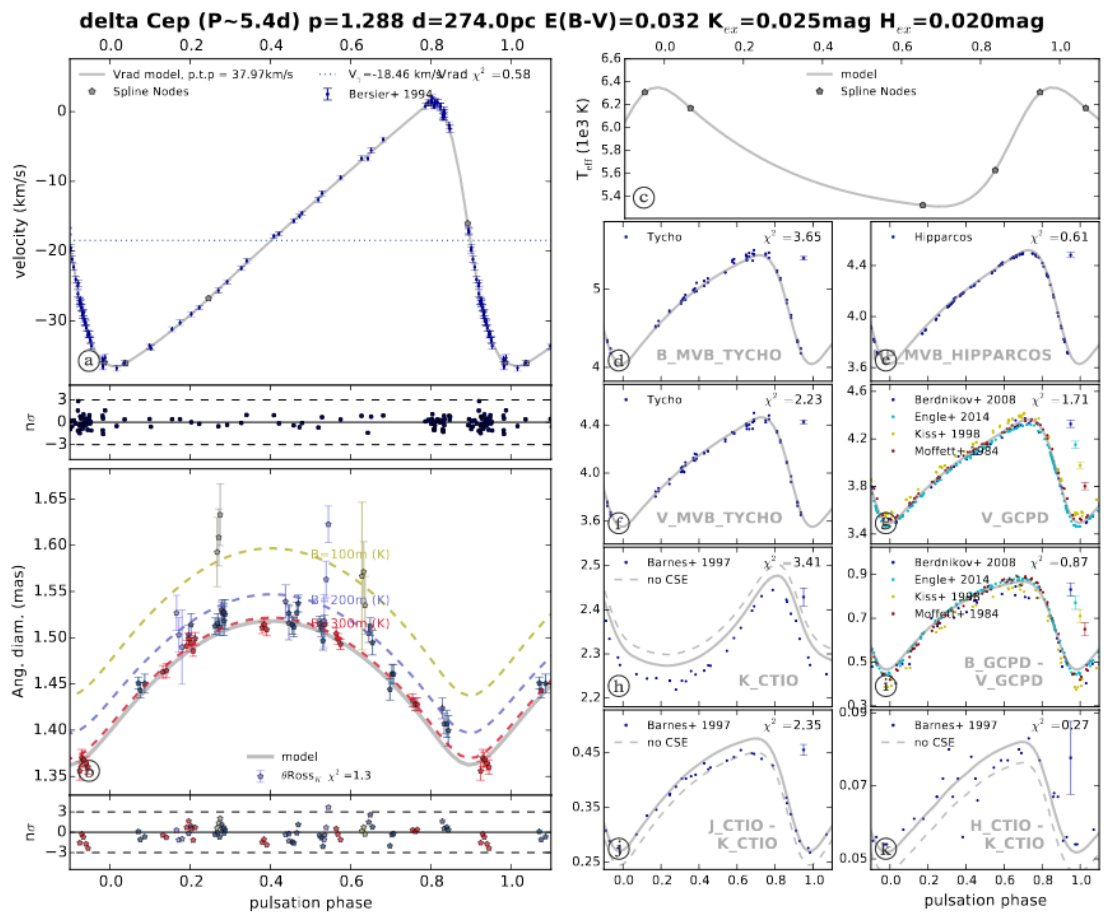

Figure 108. SpectroPhoto-Interferometry of Pulsating Stars (SPIPS) is a version of the BaadeWesselink distance determination method that combines observations of different types (photometry, velocimetry and interferometry) to constrain the parameters of a quasi-static model of pulsation of the Cepheid (here $\delta$ Cep), and in particular its distance [46]. 
Interferometry plays a key role in the calibration of distance scales in the universe, and this at different levels. Indeed, to understand the nature of dark energy, we must measure the rate of expansion of the universe, that is to say the Hubble constant $\left(H_{0}\right)$, with a good precision, i.e. better than $2 \%$. The two main methods for doing this, the study of the cosmic background radiation and the determination of distances in the universe show significant disagreements, which we refer to as the 'tension'. One of the ways to solve this tension is to better calibrate the period-luminosity relation (PL) of Cepheids: what is its zero point? Is it sensitive to metallicity? Can we reduce the dispersion of the relationship? The method based on eclipsing binaries has recently been used to reach the $2.2 \%$ precision on the LMC distance [47], which makes it possible to constrain the zero point of the PL relation and to study the effect of metallicity. The eclipsing binary method is based on a very simple concept: the linear radii of the two components of the eclipsing binary are deduced from photometric and spectroscopic observations, while the corresponding angular diameters are calculated from a surface brightness-color relationships, which is precisely calibrated by interferometry. As part of the international project Araucaria ${ }^{6}$, the current goal is to reach $1 \%$ on the accuracy on these relations [48, 49], especially for KIII-type giant stars (such as those found in LMC eclipsing binaries). This will allow to reach the $1 \%$ precision on the distance of LMC. Similar efforts aim to calibrate the surface-brightness color relationships for early types stars $(\mathrm{O}, \mathrm{A}$, B) [45], that we find in M31 and M33 eclipsing binaries, two key galaxies for the calibration of the $P L$ relationship.

Moreover, applying the Baade-Wesselink method (see the recent SPIPS version, Fig. 108) of distance determination to the Cepheids of the Magellanic clouds is now possible [50], but the projection factor and its dependence with the Cepheid pulsation period remains a keyissue, except perhaps for $\delta$ Cep, a well-understood prototypical star [51]. It is therefore crucial to refine our understanding of the atmospheric dynamics of Cepheids and their environment to determine distances in the universe. This is particularly true as the envelopes of the Cepheids could have an non-universal effect on the dispersion of the period-luminosity relation. Cepheid envelopes were detected by infrared interferometry [52], but rather surprisingly, an envelope around $\delta$ Cep was recently detected in the visible range by the VEGA/CHARA interferometer contributing to $7 \%$ of the flux of the star [53]. An effort is currently done to understand the structure and the physico-chemical nature of these envelopes, as well as their distribution in the instability strip.

\subsection{Young stellar objects}

Finally, we will discuss the contribution of imagery and high angular resolution to the study of young stars. These objects are complex with most of the time a circumstellar disc, whose material is accreted toward the central star, then ejected and collimated into a jet. We thus try to understand the geometrical structure of the disc, its physico-chemical nature, as well as the links with the planetary formation. For these young stellar objects the multi-wavelength approach is particularly suitable: the outer part of the disk is probed by radio interferometry, with ALMA for example (see the section on young objects), the medium-infrared allows to determine the molecular composition of the heart of the disc (soon with MATISSE / VLTI), while the near infrared ( $\mathrm{K}$ band) and the ionized gas ( $\mathrm{Br} \gamma$, AMBER/VLTI line) allow to prode the inner edge of the dust disc. Concerning the visible domain, it allows us to get even closer to the star (ionized gas probed by the line $\mathrm{H} \alpha$ with VEGA/CHARA), while the UV emission informs us about the magneto-spheric accretion and energy processes related to the jet. Here are some examples:

\footnotetext{
${ }^{6}$ https://araucaria.camk.edu.pl/
} 


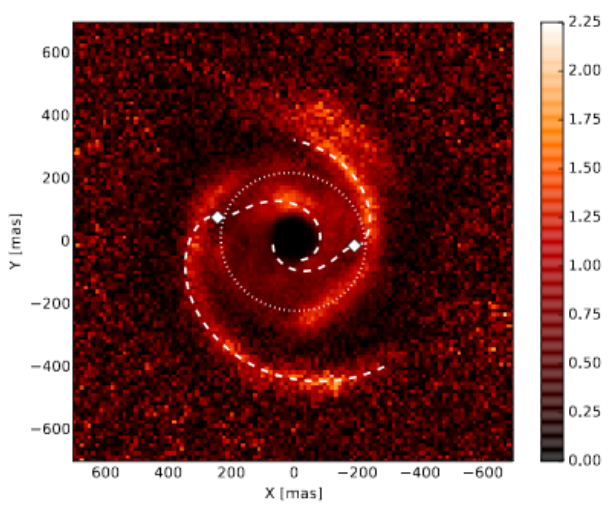

Figure 109. Polarized light image of the SPHERE/VLT protoplanetary disk MWC758 in the infrared. The two diamonds correspond to planets. The dotted ellipse corresponds to a projected circle with a radius of 250 mas [54].

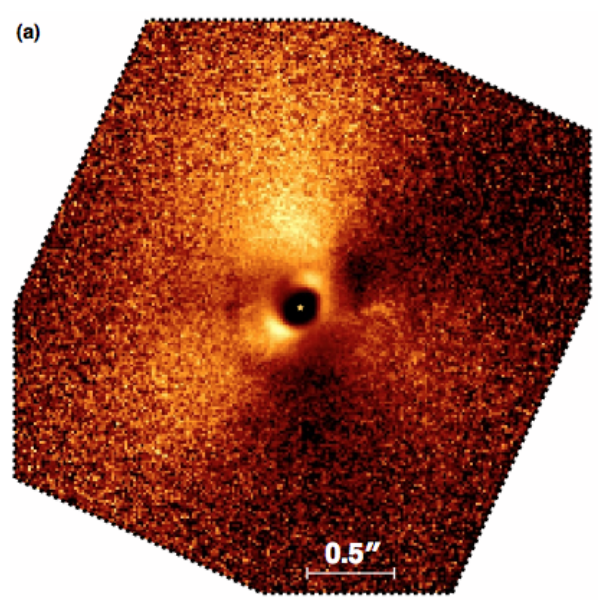

Figure 110. HD100546 observations of ZIMPOL/SPHERE in polarized light with a resolution for this object of 2 au. Structures in the discs are clearly detected and can be the understood as an interaction between the disk and planets [55].

- First, let us take the example of VEGA data on CHARA obtained in different spectral channels of the $\mathrm{H} \alpha$ emitting line of the Herbig Ae/Be star AB Aur [57], which is an excellent tracer of the geometry and kinematics of the ionized gas just below the inner edge of the disk of dust (0.05- $0.15 \mathrm{ua})$, and which indeed showed that there was an accretion wind on the innermost part of the disk.

- Then, let's continue our way out with [58] that probed the inner part of the HD50138 disk, a pre- or post-primary B[e] star using AMBER/VLTI observations in the Br $\gamma$ line. These observations show that the zone of emission of the ionized hydrogen gas is closer to the star ( $<3$ ua) than the emission due to the dust continuum, which helps to constrain the geometry of the object. These observations also show that the disk has a Keplerian rotation. 


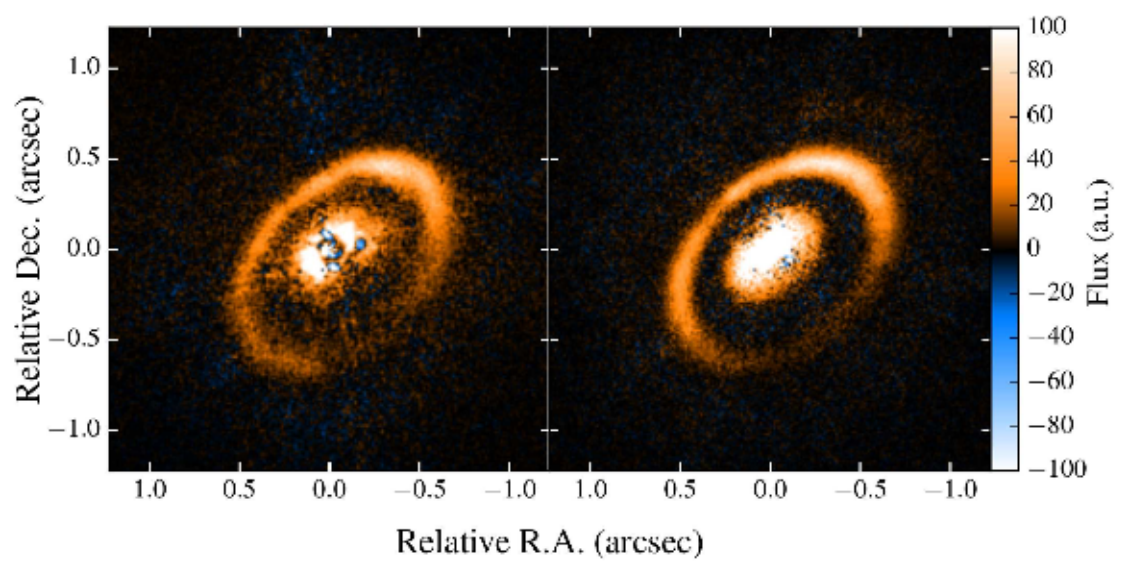

Figure 111. Left, observations ZIMPOL/SPHERE of HD163296 in polarized light, and corresponding model on the right (see [56] for more details).

- Recently, thanks to its sensitivity, the GRAVITY instrument of the VLTI [59] has been able to probe the emission $\mathrm{Br} \gamma$ at scales lower than the astronomical unit. [60] observed $\mathrm{S} \mathrm{CrA \text {, }}$ a double classical T Tauri star located at a distance of $130 \mathrm{pc}$. The continuum (K band) allows to resolve two disks of radius 0.1 ua each, having a tilt and a very similar position angle, which suggests that they stem from the fragmentation of a single disk. The visibility signature as a function of the wavelength makes it possible to detect and locate spatially the emissions of the $\mathrm{He} \mathrm{I}$ and $\mathrm{Br} \gamma$ lines, showing an inverse $\mathrm{P}$ Cygni profile, characteristic of an accreting gas. The $\mathrm{Br} \gamma$ emission is compact with a radius of $\sim 0.06$ ua, about twice as large as the truncation radius of the inner disk, and traces mainly a wind. In the coming years other observations of a sample of $\mathrm{T}$ Tauri stars with different masses, ages and disk properties are planned with GRAVITY.

- This type of statistical approach is already underway with the PIONIER/VLTI observation of $21 \mathrm{~T}$ Tauri type stars. [61] have shown that for 13 of them there is a disc, whose scattered light (in band $\mathrm{H}$ ) extends beyond 3 ua (at $150 \mathrm{pc}$ ). Each object looks different and an analysis job is in progress.

- Finally, on a larger scale, many results have been obtained by SPHERE/VLT. First, [54] unveiled an extremely complex structure within the MWC 758 protoplanetary disk between 26ua and 148ua, using observations in infrared polarized light. These SPHERE/VLT observations confirm the presence of two spirals, whose origin is attributed to two planets (see Fig. 109). Let us also mention the results obtained with SPHERE on the protoplanetary disk LkCa15 [62], as well as the results obtained by [55] on HD100546 (see Fig. 110), or HD163296 [56] (see Fig. 109) and HD169142 [41].

- Concerning the external parts of the protoplanetary disks, which are mainly probed by ALMA, we refer the reader to the following publications (to be specified).

\subsection{The prototypic star $\beta$ Lyrae}

In this short section, we would like to show a recent result [63] concerning the interacting binary $\beta$ Lyrae which perfectly illustrates the relevance of a global approach on a prototypical 
object. $\beta$ Lyrae is an extremely complex object studied since 1830 and which has made the subject of more than 850 publications. The combination of a rich bibliographic heritage, new photometric and interferometric data (NPOI, CHARA/MIRC and VEGA) associated with a complex model (SHELLPEC) allowed to provide a coherent understanding of the object, whose figure 112 gives a representation. The coordination of photometric, spectroscopic and interferometric observations has permitted a global modeling from UV to far IR. The structure of the accretting disk, its temperature profile demonstrate that it is not in hydrostaic equilibrium. A mass transfer is thus clearly established. Authors have also demonstrated that a hot spot appears on the disk and that it is clearly related to the mass transfer from the donor.

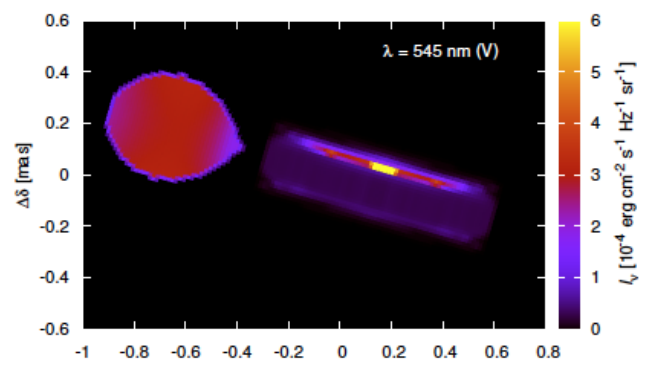

Figure 112. Three-dimensional model of $\beta$ Lyrae (SHELLPEC), constrained by photometric and interferometric data NPOI, MIRC and VEGA on CHARA [63]

So far we have been interested in 'stellar zoology' in high angular resolution, i.e. in their activity (granulation, rotation, pulsation) or their environment (Be and/or protoplanetary disks, envelope of AGB or Cepheids). We will now enter another field, that is the fundamental parameters of stars and planets.

\subsection{Interferometry, asteroseismology and fundamental stellar and planetary parameters}

The Figure 113 (shorty $R$. Ligi), illustrates the path and synergy between interferometry, asteroseismology and the precise and exact determination of stellar and planetary parameters.

Let's start at the top of the figure. Interferometry requires surface-brightness color relationships (or equivalent) to determine the angular diameter of its calibrators, and hence, to calibrate the visibility measurements corresponding to the star to be studied ${ }^{7}$. A remarkable work has been to provide a catalog (the JSDC: 'The Stellar JMMC Diameter Catalog' version 2) which contains the estimated angular diameter for 465877 stars, with a median error of $1.5 \%$ and possible bias of the order of $2 \%$ [64]. Nevertheless, it must be kept in mind that the activity of the star (wind, environment, spots, granulation, rotation) has an effect on the estimate of the diameter of the calibrator and/or the star science ${ }^{8}$. The rapid rotation has for example an effect on the estimate of the diameter of stars $\mathrm{O}, \mathrm{A}, \mathrm{B}$ of the order of a few percent $[45,65]$.

It should also be noted that the conversion between the "uniform" angular diameter $\theta_{\text {UD }}$ and "darkened" $\theta_{\mathrm{LD}}$, i.e. taking into account the limb-darkening of the star requires a model of the atmosphere of the star. Efforts are currently done to quantify the effect of a 1D or 3D

\footnotetext{
${ }^{7}$ These are precisely the same relationships that are used to determine the distance of eclipsing binaries, see section above

${ }^{8}$ It should be kept in mind that the accuracy on the diameter of the interferometric calibrator is not linearly related to the accuracy on the diameter of the star science
} 


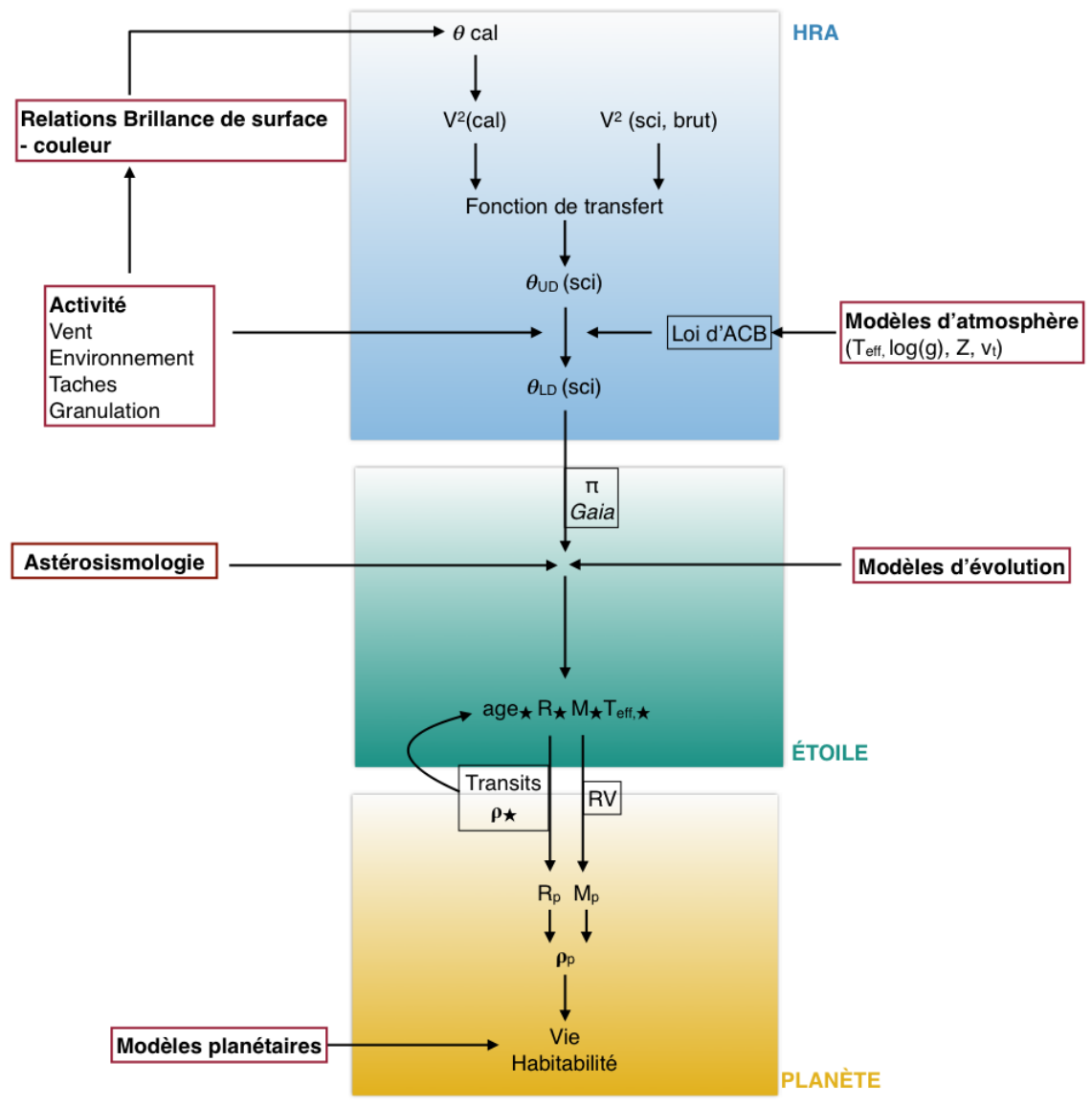

Figure 113. Interferometry, asteroseismology and fundamental stellar and planetary parameters. This figure illustrates how interferometric angular diameters, combined with precise and accurate parallax measurements (Gaia), constrain asteroseismic models, which in turn (via current and future planetary transient measurements with PLATO) allow to deduce the radius of the planet from the radius of the star.

model on the interferometric estimation of the angular diameter, especially for the giant [6668]. This work is particularly interesting as it is now possible, for only a few stars, to measure directly the limb-darkening of a star by interferometry. Limb-darkening is therefore not assumed (based on a 1D or 3D atmosphere model), but directly measured using precise and accurate visibility measurements at high spatial frequencies. Note at this level the remarkable result obtained on $\alpha$ Cen A \& B with the PIONIER instrument on the VLTI [69], which made it possible to measure the limb-darkening of the star. These measurements indicate lower limb-darkening than the values predicted by the 1D or 3D models (see Fig. 114).

Once the angular diameter of the star is determined independently by interferometry, it is then possible to combine it with a measure of Gaia parallax (for example) to deduce the radius of the star and then compare it with a radius estimate from asteroseismic measure- 


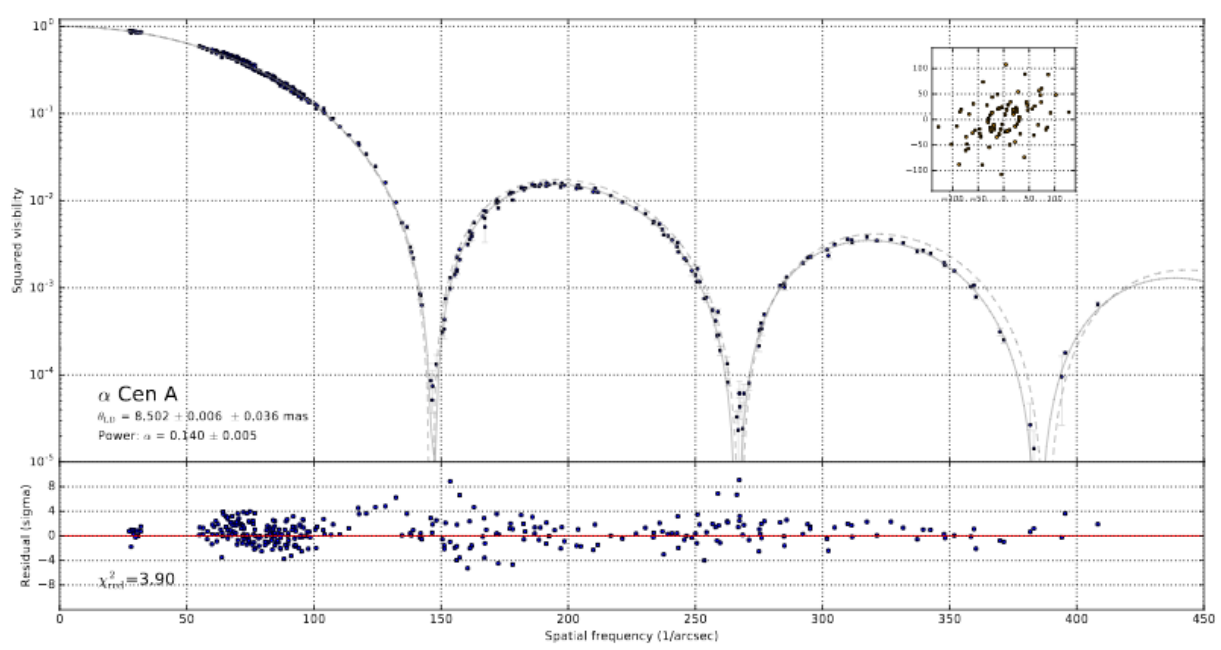

Figure 114. The limb-darkening of $\alpha$ Cen A measured by the Pionier instrument on the VLTI. The dotted line corresponds to a uniform disk model of the star, while the solid line corresponds to a limbdarkened disk model. In paper [69], the authors find a diameter of $\theta_{\mathrm{LD}}=8.502 \pm 0.038$ mas $(0.43 \%$ accuracy) using an analytic power law for the limb-darkening, which is then compared to $1 \mathrm{D}$ or $3 \mathrm{D}$ atmospheric models of limb-darkening.

ments [70, 71]. The agreement is generally good, but gaps are currently observed for giant stars. There is also another way to proceed, without using parallaxes. Asteroseismic scaling relationships [72, 73] rely the mass and radius of the star to the actual temperature, in an independent way. Thus, if one determines the temperature of the star, by spectroscopic measurements for example (historical approach), one obtains the asteroseismic mass and radius. The interferometric diameter can here play an important role, since the temperature can be deduced directly from the bolometric flux of the star and the angular diameter. The problem comes down to compare the temperatures derived by spectroscopy and interferometry, respectively. This is what is currently done on roAp type stars, which have also spots due to their magnetism [74], which are known to alter the derived temperature. Since the roAp have extremely small angular diameters, the VEGA/CHARA instrument, whose spatial resolution is one of the largest in the world (see Figure 104) has been used to measure their angular diameter. For instance, it allowed to measure the angular diameter of 78 Vir and HD24712, which have indeed the smallest diameters ever measured, respectively $\theta_{\mathrm{LD}}=0.346 \pm 0.006$ mas [75] and $\theta_{\mathrm{LD}}=0.335 \pm 0.009$ mas [74] (see Fig. 115).

The determination of the effective temperature of the stars is thus a key issue, which is particularly important when considering metal poor stars. These stars are excellent tracers in galactic archeology, which brings constraints on the structure and evolution of galaxies. As an example, we can mention the star HD140283 observed again with VEGA/CHARA because of its extremely small diameter: $\theta_{\mathrm{LD}}=0.353 \pm 0.013$ mas. These observations were used to compare the spectroscopic (based in particular on the $\mathrm{H} \alpha$ spectral line) and interferometric temperatures, while stellar evolution models allow us to deduce the mass and the age of the star. Using a 3D atmosphere model and a CESAM2k stellar evolution model, remarkable accuracies of $1.8 \%$ and $7.4 \%$ on the mass and age of the star were obtained [76]. Note that this star is particularly interesting because it is part of the 'benchmark' stars of Gaia. 


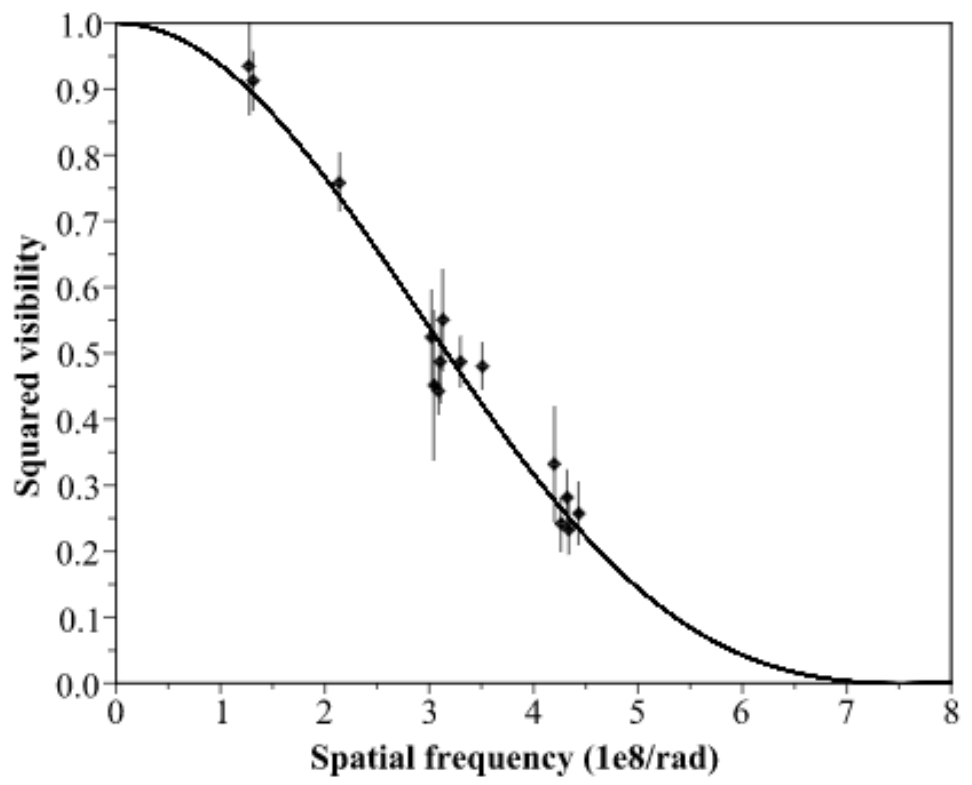

Figure 115. Angular diameter measurement of the roAp 78 Vir done with the VEGA/CHARA instrument. This is one of the smallest angular diameters ever measured, $\theta_{\mathrm{LD}}=0.346 \pm 0.006$ mas [75].

The determination of stellar parameters is an interesting subject in itself, because the stars are the constitutive elements of galaxies, but also because these stellar parameters are indispensable for determining the exo-planetary parameters. Indeed, if we know the ratio of stellar and planetary radii by an exo-planetary transit measurement from space missions, CoRoT, Kepler, K2, TESS or soon PLATO, then it is enough to know the radius of the star to deduce the radius of the planet, and therefore its density. Since a $1 \%$ accuracy is expected on the transits, an accuracy of $1 \%$ on the star radius would be required to deduce the planetary radius at $1 \%$. In this context, the measurements of angular diameters of 10 exoplanet host stars, including one having a transit $(55 \mathrm{Cnc})$, is an important first step to consider [41]. The average accuracy on the derived angular diameters is of about $2 \%(1.6 \%$ for $55 \mathrm{Cnc})$.

This synergy combining interferometry, asteroseismology, also related to spatial missions Gaia and PLATO, is something very interesting that should be followed in the coming decade.

\subsection{Doppler Imaging and Hot Jupiter Detection}

'Hot Jupiters' orbit on average 100 times closer to their star than Jupiter around the Sun. But how and when in their history do they migrate so close to their star? [77] have just discovered a very young hot Jupiter in the immediate vicinity of a young T Tauri star, barely 2 million years old. But the detection of planets around very young stars proves to be a real challenge, because these stars are very active: their intense magnetic activity disturbs the light emitted by the star significantly. One of the team's prowess was to separate the signal due to the activity of the star from the one generated by the planet. By removing the effect of activity on the ESPaDOnS and Narval velocity curves using Doppler imaging techniques, the team detected a periodic signal of $4.93 \pm 0.05$ days and a semi-amplitude of $75 \pm 11$ $\mathrm{m} / \mathrm{s}$. This signal completely disconnected from the rotation of the V830 Tau star (2.741 days) 


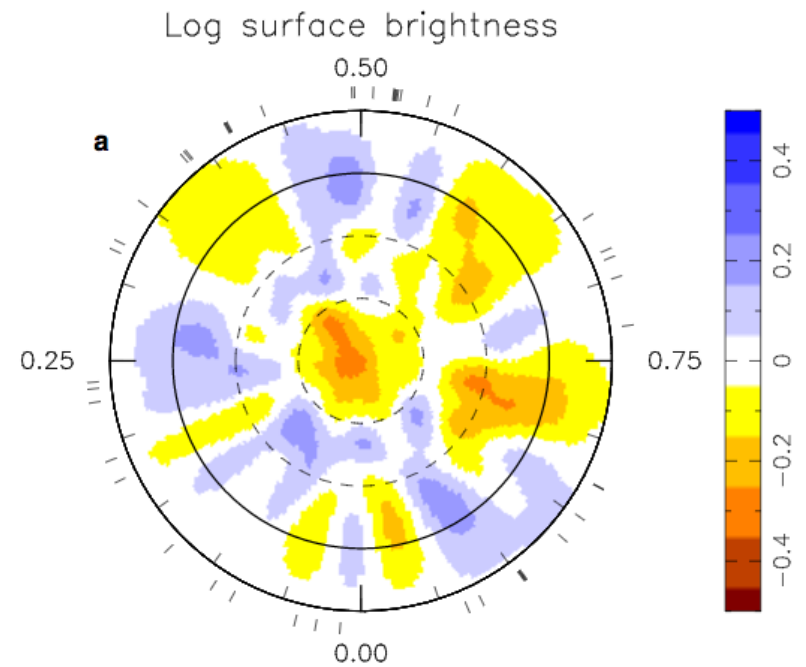

Figure 116. Brightness distribution of the T Tauri V830 Tau obtained from a fit of LSD ('Least Squared Deconvolution' technique) profiles of ESPaDOnS and Narval, according to the Doppler imaging method [77]. The cold and hot spots are represented in brown and blue, respectively.

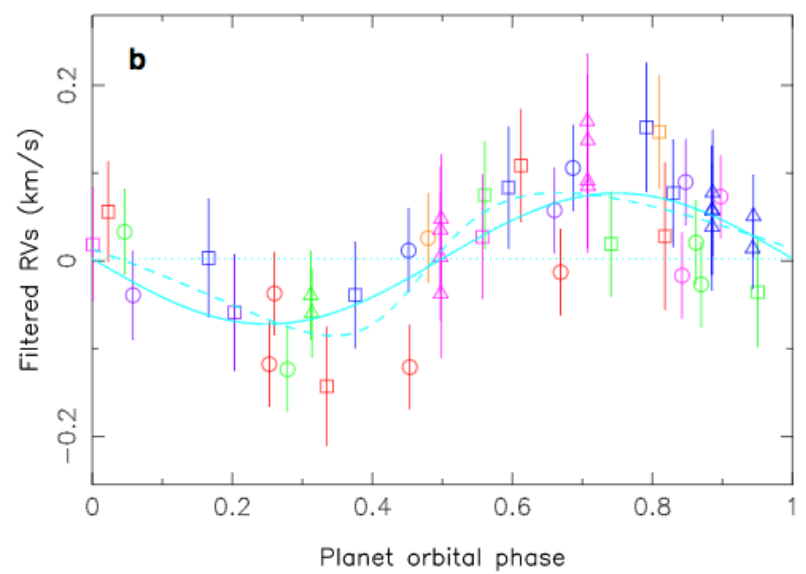

Figure 117. Radial velocity curves of a hot Jupiter around V830 Tau. The Doppler Imaging technique was used to determine the activity of the star, whose velocimetric signature was removed from the ESPaDOnS and Narval observations. The residual radial velocity curve is attributed to the presence of a hot Jupiter [77].

is thus attributed to the presence of a mass planet $0.77 \pm 0.15 \mathrm{Ms}$ orbiting at a distance of $0.057 \pm 0.001$ of the host star. This discovery thus confirms that early migration within the disk is possible in the case of giant planets, the other hypothesis being a migration arriving erratically later in the history of the system by the interplay between planets. 
Here we see the power of Doppler imaging, which can be combined (if the object is bright enough) with interferometric measurements as we have already mentioned in the case of red giants and supergiants.

\subsection{Detection of the gravitational redshift in the orbit of the star S2 near the Galactic center massive black hole}

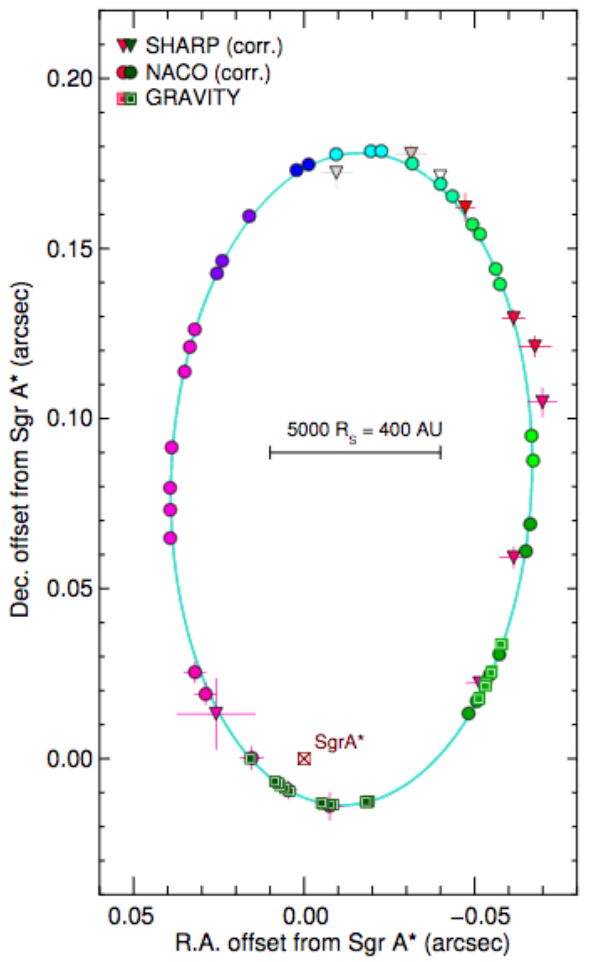

Figure 118. Verification of Einstein's theory of General Relativity near the supermassive black hole (Sgr A *) by the GRAVITY/VLTI instrument.

Before concluding, we would like to emphasize a recent spectacular result obtained by the team in charge of the GRAVITY instrument at the VLTI. By accurately determining the trajectory of the S2 star in orbit around the supermassive black hole located at the center of the Milky Way (Sgr A*), measurements of the GRAVITY instrument (astrometry) made it possible to determine the gravitational redshift of the S2 star and thus confirm the theory of relativity of Einstein [78]. Indeed, at periastron, i.e. at 120 ua of the black hole or 1400 Schwarzschild' radii, the star has an orbital velocity of $7640 \mathrm{~km} / \mathrm{s}$, a velocity sufficiently large for the effects of relativity to become measurable (see Fig. 118).

\section{Steps to the future}

Optical interferometry has made important progresses these last three decades after the pioneering works of the first instruments. It is interesting to compare these progresses with the same ones done in radio-interferometry (see Fig. 119). 
If one looks at the progression of the publications in optical interferometry, it can be understood that this domain mimics the radio-interferometry developments with a shift of thirty years in fact!

In the next decade, we can anticipate several synergies:

- direct visible imaging and radio interferometry that allow to superimpose images at different wavelengths but with the same angular resolution (case of the AGB star L2 Pup);

- interferometric and Doppler imaging, which both allow to see the spots on the surface of stars (case of Betelgeuse), as well as Doppler imaging and velocimetry for the detector of exo-Jupiters (case of the Tauri V830 Tau);

- direct imaging and interferometry: for example, detection of exoplanets by imaging with SPHERE and determination of the interferometric diameter of the host star to determine the age of the system (case of GJ504);

- the interferometry in the continuum and in a spectral line ( $\mathrm{H} \alpha$ for example) to probe both the geometry and the kinematics of the studied object (case of the Be star $\phi$ Per, but also of young stars);

- interferometry and asteroseismology for the determination of stellar and planetary parameters.

Beyond these synergies, the road will continue for sure and we will, in the future, reach facilities with a large number of sub-apertures and an incredible imaging power. An international group is developing the conceptual studies for a project called Planet Finder Imager [79]. However the complexity of such developments because of the current technology and concepts used in the current facilities may prevent the astronomical community to see these facilities operating one day. Other alternatives are considered, such as intensity interferometry or space developments in order to simplify the implementation technology.

We are also developing a demonstrator of a new kind of recombination based on the principle of the Arecibo radio telescope. The idea is to replace the pointing primary mirror (or mirrors in the case of an interferometer) by a fixed primary mirror and a moving gondola acquiring the moving image on the focal sphere. This has proven to be well adapted at radio wavelengths with the long life of the Arecibo radio telescope. The concept has even been improved recently with a continuously deformable primary mirror of $500 \mathrm{~m}$ in diameter (the FAST Chinese radio telescope [84]) to permanently match the required parabola primary to avoid the limits generated by the spherical aberration of a spherical primary mirror. The very interesting feature of such a configuration is that it permits to avoid the implementation of long delay lines and that it highly simplifies the transfer of beams from the collecting sub apertures to the combiner. We have started the development of a demonstrator [85] in a valley of the Southern Alps, as represented on Fig. 120.

Although the first results of this prototype are highly encouraging in terms of control and accuracy of tracking, the way is still long before a full implementation of a complete system. A space-based or moon-version of such an ambitious hypertelescope is maybe the correct development that will have to be done in the future. However the current facilities like the VLTI or CHARA have still a great potential of developments in the coming decade, with improved adaptive optics systems, fringe sensor, additional telescopes or additional wavebands. And, as said previously, the need for high angular resolution beyond the diffraction limit of single telescopes is clearly the main driver of the future development of this observing technique.

\section{References}

[1] A.A. Michelson, ApJ 51, 257 (1920) 

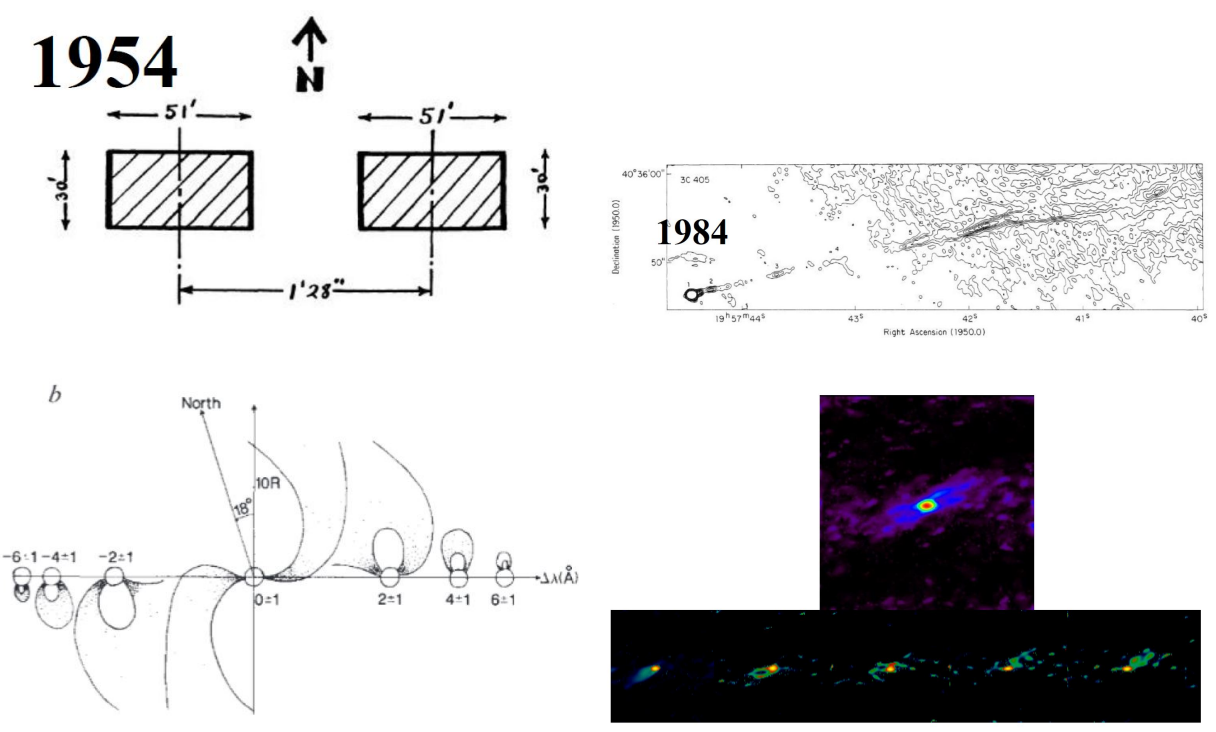

Figure 119. Comparison of the representation of a radio source between 1954 and 1984 (top row: from Jennison [80] and Perley [81]), and in the optical domain between 1989 and 2015 [82, 83]

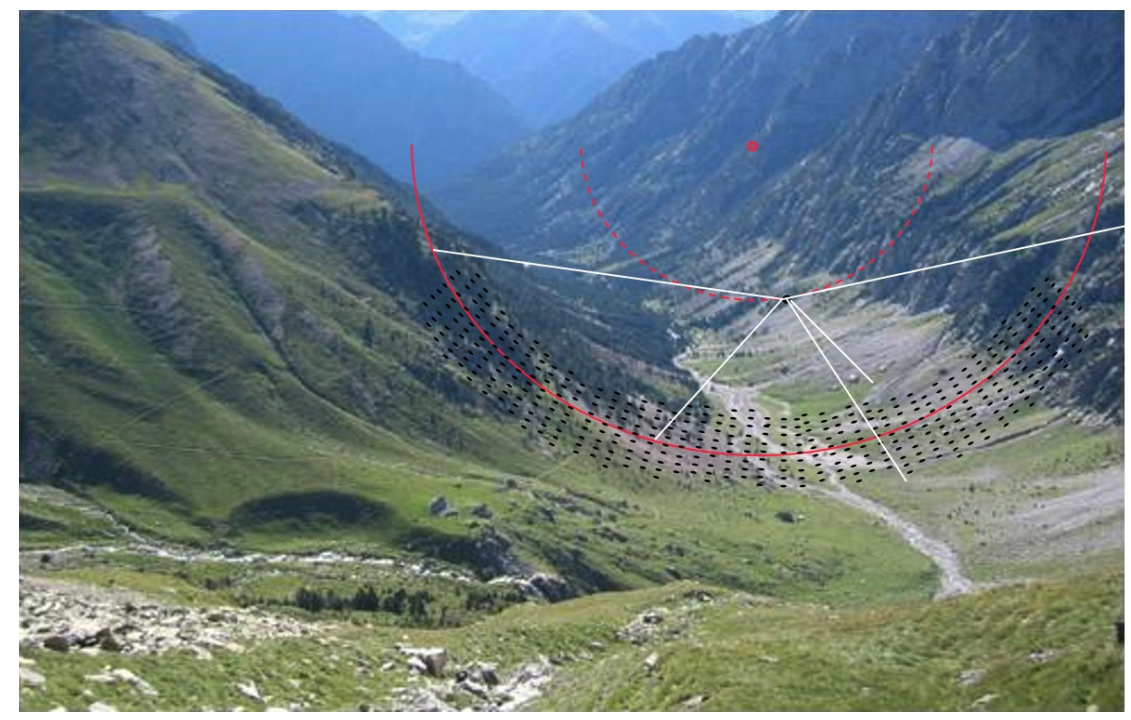

Figure 120. Representation of the installation of the Ubaye Hypertelescope prototype in a valley of the Southern Alps. The primary mirror, made of small segments of $20 \mathrm{~cm}$ in diameter, is fixed on the ground and matches a large sphere with $202 \mathrm{~m}$ in radius of curvature. A focal gondola located $101 \mathrm{~m}$ above the primary mirror is located on the focal sphere and is moved to permanently stay at the location of the moving image of the star. 
[2] H. Fizeau, CRAS (1868)

[3] A. Labeyrie, ApJ 196, L71 (1975)

[4] J.W. Goodman, Statistical Optics (1985)

[5] R.H. Brown, Nature 218, 637 (1968)

[6] A. Labeyrie, A\&A Sup.Ser. 118, 517 (1996), astro-ph/9602093

[7] O. Lardière, F. Martinache, F. Patru, MNRAS 375, 977 (2007), astro-ph/0612074

[8] F. Patru, D. Mourard, O. Lardière, S. Lagarde, MNRAS 376, 1047 (2007), astro-ph/0701467

[9] A. Labeyrie, D. Mourard, F. Allouche, R. Chakraborthy, J. Dejonghe, A. Surya, Y. Bresson, C. Aime, D. Mary, A. Carlotti, Concept study of an Extremely Large Hyper Telescope (ELHyT) with $1200 \mathrm{~m}$ sparse aperture for direct imaging at 100 micro-arcsecond resolution, in Optical and Infrared Interferometry III (2012), Vol. 8445, p. 844512

[10] A. Labeyrie, F. Allouche, D. Mourard, F. Bolgar, R. Chakraborty, J. Maillot, N. Palitzyne, J.R. Poletti, J.P. Rochaix, R. Prud'homme et al., Construction of a 57m hypertelescope in the Southern Alps, in Optical and Infrared Interferometry III (2012), Vol. 8445 , p. 844511

[11] M. Tallon, I. Tallon-Bosc, A\&A 253, 641 (1992)

[12] P. Berio, D. Mourard, F. Vakili, J. Borgnino, A. Ziad, Journal of the Optical Society of America A 14, 114 (1997)

[13] L. Koechlin, P.R. Lawson, D. Mourard, A. Blazit, D. Bonneau, F. Morand, P. Stee, I. Tallon-Bosc, F. Vakili, Applied Optics 35, 3002 (1996)

[14] M. Gai, S. Menardi, S. Cesare, B. Bauvir, D. Bonino, L. Corcione, M. Dimmler, G. Massone, F. Reynaud, A. Wallander, The VLTI fringe sensors: FINITO and PRIMA FSU, in New Frontiers in Stellar Interferometry, edited by W.A. Traub (2004), Vol. 5491 of Proc. of the SPIE Conference, p. 528

[15] Gravity Collaboration, R. Abuter, M. Accardo, A. Amorim, N. Anugu, G. Ávila, N. Azouaoui, M. Benisty, J.P. Berger, N. Blind et al., A\&A 602, A94 (2017), 1705.02345

[16] D. Mekarnia, J. Gay, Journal of Optics 20, 131 (1989)

[17] A.R. Thompson, J.M. Moran, G.W. Swenson, Jr., Interferometry and Synthesis in Radio Astronomy, 3rd Edition (2017)

[18] E.V. Garcia, M.W. Muterspaugh, G. van Belle, J.D. Monnier, K.G. Stassun, A. Ghasempour, J.H. Clark, R.T. Zavala, J.A. Benson, D.J. Hutter et al., PASP 128, 055004 (2016), 1601.00036

[19] B. Lazareff, J.B. Le Bouquin, J.P. Berger, A\&A 543, A31 (2012)

[20] B. Lopez, S. Lagarde, W. Jaffe, R. Petrov, M. Schöller, P. Antonelli, U. Beckmann, P. Berio, F. Bettonvil, A. Glindemann et al., The Messenger 157, 5 (2014)

[21] J. Sturmann, T. Ten Brummelaar, L. Sturmann, H.A. McAlister, Dual three-way infrared beam combiner at the CHARA Array, in Society of Photo-Optical Instrumentation Engineers (SPIE) Conference Series (2010), Vol. 7734 of Presented at the Society of Photo-Optical Instrumentation Engineers (SPIE) Conference

[22] N.J. Scott, R. Millan-Gabet, E. Lhomé, T.A. Ten Brummelaar, V. Coudé Du Foresto, J. Sturmann, L. Sturmann, Journal of Astronomical Instrumentation 2, 1340005 (2013)

[23] J.D. Monnier, M. Zhao, E. Pedretti, N. Thureau, M. Ireland, P. Muirhead, J.P. Berger, R. Millan-Gabet, G. Van Belle, T. ten Brummelaar et al., Imaging the surface of Altair and a MIRC update, in Society of Photo-Optical Instrumentation Engineers (SPIE) Conference Series (2008), Vol. 7013 of Presented at the Society of Photo-Optical Instrumentation Engineers (SPIE) Conference 
[24] M.J. Ireland, A. Mérand, T.A. ten Brummelaar, P.G. Tuthill, G.H. Schaefer, N.H. Turner, J. Sturmann, L. Sturmann, H.A. McAlister, Sensitive visible interferometry with $P A V O$, in Society of Photo-Optical Instrumentation Engineers (SPIE) Conference Series (2008), Vol. 7013 of Presented at the Society of Photo-Optical Instrumentation Engineers (SPIE) Conference

[25] D. Mourard, J.M. Clausse, A. Marcotto, K. Perraut, I. Tallon-Bosc, P. Bério, A. Blazit, D. Bonneau, S. Bosio, Y. Bresson et al., A\&A 508, 1073 (2009)

[26] D. Mourard, P. Bério, K. Perraut, R. Ligi, A. Blazit, J.M. Clausse, N. Nardetto, A. Spang, I. Tallon-Bosc, D. Bonneau et al., A\&A 531, A110 (2011)

[27] A. Labeyrie, Exo-Earth Imager for Exoplanet Snapshots with Resolved Detail, in Working on the Fringe: Optical and IR Interferometry from Ground and Space, edited by S. Unwin, R. Stachnik (1999), Vol. 194 of Astronomical Society of the Pacific Conference Series, p. 350

[28] C. Paladini, F. Baron, A. Jorissen, J.B. Le Bouquin, B. Freytag, S. van Eck, M. Wittkowski, J. Hron, A. Chiavassa, J.P. Berger et al., Nature 553, 310 (2018)

[29] M. Montargès, P. Kervella, G. Perrin, A. Chiavassa, J.B. Le Bouquin, M. Aurière, A. López Ariste, P. Mathias, S.T. Ridgway, S. Lacour et al., A\&A 588, A130 (2016), 1602.05108

[30] M. Montargès, A. Chiavassa, P. Kervella, S.T. Ridgway, G. Perrin, J.B. Le Bouquin, S. Lacour, A\&A 605, A108 (2017), 1705.07829

[31] A. Chiavassa, R. Norris, M. Montargès, R. Ligi, L. Fossati, L. Bigot, F. Baron, P. Kervella, J.D. Monnier, D. Mourard et al., A\&A 600, L2 (2017), 1703.02406

[32] R.M. Roettenbacher, J.D. Monnier, H. Korhonen, A.N. Aarnio, F. Baron, X. Che, R.O. Harmon, Z. Kővári, S. Kraus, G.H. Schaefer et al., Nature 533, 217 (2016), 1709.10107

[33] B. Freytag, S. Liljegren, S. Höfner, A\&A 600, A137 (2017), 1702.05433

[34] M. Aurière, A. López Ariste, P. Mathias, A. Lèbre, E. Josselin, M. Montargès, P. Petit, A. Chiavassa, F. Paletou, N. Fabas et al., A\&A 591, A119 (2016), 1605.04702

[35] B. Tessore, A. Lèbre, J. Morin, P. Mathias, E. Josselin, M. Aurière, A\&A 603, A129 (2017), 1704.07761

[36] A. Lèbre, M. Aurière, N. Fabas, D. Gillet, F. Herpin, R. Konstantinova-Antova, P. Petit, A\&A 561, A85 (2014), 1310. 4379

[37] D. Mourard, J.M. Clausse, A. Marcotto, K. Perraut, I. Tallon-Bosc, P. Bério, A. Blazit, D. Bonneau, S. Bosio, Y. Bresson et al., A\&A 508, 1073 (2009)

[38] P. Kervella, M. Montargès, E. Lagadec, S.T. Ridgway, X. Haubois, J.H. Girard, K. Ohnaka, G. Perrin, A. Gallenne, A\&A 578, A77 (2015), 1511.04448

[39] P. Kervella, W. Homan, A.M.S. Richards, L. Decin, I. McDonald, M. Montargès, K. Ohnaka, A\&A 596, A92 (2016), 1611.06231

[40] M. Bonnefoy, K. Perraut, A.M. Lagrange, P. Delorme, A. Vigan, M. Line, L. Rodet, C. Ginski, D. Mourard, G.D. Marleau et al., ArXiv e-prints (2018), 1807.00657

[41] R. Ligi, O. Creevey, D. Mourard, A. Crida, A.M. Lagrange, N. Nardetto, K. Perraut, M. Schultheis, I. Tallon-Bosc, T. ten Brummelaar, A\&A 586, A94 (2016), 1511.03197

[42] G. Dalla Vedova, F. Millour, A. Domiciano de Souza, R.G. Petrov, D. Moser Faes, A.C. Carciofi, P. Kervella, T. Rivinius, A\&A 601, A118 (2017), 1703.02839

[43] D. Mourard, J.D. Monnier, A. Meilland, D. Gies, F. Millour, M. Benisty, X. Che, E.D. Grundstrom, R. Ligi, G. Schaefer et al., A\&A 577, A51 (2015), 1503.03423

[44] A. Domiciano de Souza, P. Kervella, D. Moser Faes, G. Dalla Vedova, A. Mérand, J.B. Le Bouquin, F. Espinosa Lara, M. Rieutord, P. Bendjoya, A.C. Carciofi et al., A\&A 569, 
A10 (2014)

[45] M. Challouf, N. Nardetto, A. Domiciano de Souza, D. Mourard, H. Aroui, P. Stee, O. Delaa, D. Graczyk, G. Pietrzyński, W. Gieren, A\&A 579, A107 (2015)

[46] A. Mérand, P. Kervella, J. Breitfelder, A. Gallenne, V. Coudé du Foresto, T.A. ten Brummelaar, H.A. McAlister, S. Ridgway, L. Sturmann, J. Sturmann et al., A\&A 584, A80 (2015), 1510.01940

[47] G. Pietrzyński, D. Graczyk, W. Gieren, I.B. Thompson, B. Pilecki, A. Udalski, I. Soszyński, S. Kozłowski, P. Konorski, K. Suchomska et al., Nature 495, 76 (2013), 1303.2063

[48] D. Graczyk, P.F.L. Maxted, G. Pietrzyński, B. Pilecki, P. Konorski, W. Gieren, J. Storm, A. Gallenne, R.I. Anderson, K. Suchomska et al., A\&A 581, A106 (2015), 1508.03188

[49] A. Gallenne, G. Pietrzyński, D. Graczyk, N. Nardetto, A. Mérand, P. Kervella, W. Gieren, S. Villanova, R.E. Mennickent, B. Pilecki, ArXiv e-prints (2018), 1806.09572

[50] A. Gallenne, P. Kervella, A. Mérand, G. Pietrzyński, W. Gieren, N. Nardetto, B. Trahin, A\&A 608, A18 (2017), 1708.09851

[51] N. Nardetto, E. Poretti, M. Rainer, A. Fokin, P. Mathias, R.I. Anderson, A. Gallenne, W. Gieren, D. Graczyk, P. Kervella et al., A\&A 597, A73 (2017), 1701.01589

[52] P. Kervella, W. Homan, A.M.S. Richards, L. Decin, I. McDonald, M. Montargès, K. Ohnaka, A\&A 596, A92 (2016), 1611.06231

[53] N. Nardetto, A. Mérand, D. Mourard, J. Storm, W. Gieren, P. Fouqué, A. Gallenne, D. Graczyk, P. Kervella, H. Neilson et al., A\&A 593, A45 (2016), 1609.07268

[54] M. Benisty, A. Juhasz, A. Boccaletti, H. Avenhaus, J. Milli, C. Thalmann, C. Dominik, P. Pinilla, E. Buenzli, A. Pohl et al., A\&A 578, L6 (2015), 1505.05325

[55] A. Garufi, S.P. Quanz, H.M. Schmid, G.D. Mulders, H. Avenhaus, A. Boccaletti, C. Ginski, M. Langlois, T. Stolker, J.C. Augereau et al., A\&A 588, A8 (2016), 1601. 04983

[56] G.A. Muro-Arena, C. Dominik, L.B.F.M. Waters, M. Min, L. Klarmann, C. Ginski, A. Isella, M. Benisty, A. Pohl, A. Garufi et al., A\&A 614, A24 (2018), 1802.03328

[57] K. Rousselet-Perraut, M. Benisty, D. Mourard, S. Rajabi, F. Bacciotti, P. Bério, D. Bonneau, O. Chesneau, J.M. Clausse, O. Delaa et al., A\&A 516, L1 (2010), 1005.5267

[58] L.E. Ellerbroek, M. Benisty, S. Kraus, K. Perraut, J. Kluska, J.B. le Bouquin, M. Borges Fernandes, A. Domiciano de Souza, K.M. Maaskant, L. Kaper et al., A\&A 573, A77 (2015), 1409.7394

[59] Gravity Collaboration, R. Abuter, M. Accardo, A. Amorim, N. Anugu, G. Ávila, N. Azouaoui, M. Benisty, J.P. Berger, N. Blind et al., A\&A 602, A94 (2017), 1705.02345

[60] Gravity Collaboration, R. Garcia Lopez, K. Perraut, A. Caratti O Garatti, B. Lazareff, J. Sanchez-Bermudez, M. Benisty, C. Dougados, L. Labadie, W. Brandner et al., A\&A 608, A78 (2017)

[61] F. Anthonioz, F. Ménard, C. Pinte, J.B. Le Bouquin, M. Benisty, W.F. Thi, O. Absil, G. Duchêne, J.C. Augereau, J.P. Berger et al., A\&A 574, A41 (2015), 1412.1052

[62] C. Thalmann, G.D. Mulders, M. Janson, J. Olofsson, M. Benisty, H. Avenhaus, S.P. Quanz, H.M. Schmid, T. Henning, E. Buenzli et al., ApJ Letters 808, L41 (2015), 1507.03587

[63] D. Mourard, M. Broz, J. Nemravova, P. Harmanec, J. Budaj, F. Baron, J. Monnier, G. Schaefer, H. Schmitt, I. Tallon-Bosc et al., ArXiv e-prints (2018), 1807.04789

[64] A. Chelli, G. Duvert, L. Bourgès, G. Mella, S. Lafrasse, D. Bonneau, O. Chesneau, A\&A 589, A112 (2016), 1604.07700 
[65] M. Challouf, N. Nardetto, D. Mourard, D. Graczyk, H. Aroui, O. Chesneau, O. Delaa, G. Pietrzyński, W. Gieren, R. Ligi et al., A\&A 570, A104 (2014), 1409. 1351

[66] L. Bigot, D. Mourard, P. Berio, F. Thévenin, R. Ligi, I. Tallon-Bosc, O. Chesneau, O. Delaa, N. Nardetto, K. Perraut et al., A\&A 534, L3 (2011), 1110.0985

[67] Z. Magic, A. Chiavassa, R. Collet, M. Asplund, A\&A 573, A90 (2015), 1403. 3487

[68] A. Chiavassa, A. Caldas, F. Selsis, J. Leconte, P. Von Paris, P. Bordé, Z. Magic, R. Collet, M. Asplund, A\&A 597, A94 (2017), 1609.08966

[69] P. Kervella, L. Bigot, A. Gallenne, F. Thévenin, A\&A 597, A137 (2017), 1610. 06185

[70] D. Huber, M.J. Ireland, T.R. Bedding, I.M. Brandão, L. Piau, V. Maestro, T.R. White, H. Bruntt, L. Casagrande, J. Molenda-Żakowicz et al., ApJ 760, 32 (2012), 1210.0012

[71] E.K. Baines, J.T. Armstrong, H.R. Schmitt, J.A. Benson, R.T. Zavala, G.T. van Belle, ApJ 781, 90 (2014), 1401. 3342

[72] Y. Lebreton, M.J. Goupil, J. Montalbán, How accurate are stellar ages based on stellar models?. II. The impact of asteroseismology, in EAS Publications Series (2014), Vol. 65 of EAS Publications Series, pp. 177-223, 1410.5337

[73] B. Mosser, Stellar oscillations - I - The adiabatic case, in EAS Publications Series (2015), Vol. 73 of EAS Publications Series, pp. 3-110, 1509.08077

[74] K. Perraut, I. Brandão, M. Cunha, D. Shulyak, D. Mourard, N. Nardetto, T.A. ten Brummelaar, A\&A 590, A117 (2016)

[75] K. Perraut, M. Cunha, I. Brandão, J. Loridat, D. Mourard, A. Meilland, N. Nardetto, H. McAlister, T.A. ten Brummelaar, J. Sturmann et al., A\&A 579, A85 (2015)

[76] O.L. Creevey, F. Thévenin, P. Berio, U. Heiter, K. von Braun, D. Mourard, L. Bigot, T.S. Boyajian, P. Kervella, P. Morel et al., ??jnlA\&A575, A26 (2015), 1410.4780

[77] J.F. Donati, C. Moutou, L. Malo, C. Baruteau, L. Yu, E. Hébrard, G. Hussain, S. Alencar, F. Ménard, J. Bouvier et al., Nature 534, 662 (2016), 1606.06236

[78] Gravity Collaboration, R. Abuter, A. Amorim, N. Anugu, M. Bauböck, M. Benisty, J.P. Berger, N. Blind, H. Bonnet, W. Brandner et al., A\&A 615, L15 (2018), 1807. 09409

[79] J.D. Monnier, S. Kraus, M.J. Ireland, F. Baron, A. Bayo, J.P. Berger, M. CreechEakman, R. Dong, G. Duchêne, C. Espaillat et al., Experimental Astronomy (2018)

[80] R.C. Jennison, M.K. Das Gupta, Nature 172, 996 (1953)

[81] R.A. Perley, J.W. Dreher, J.J. Cowan, ApJ Letters 285, L35 (1984)

[82] D. Mourard, I. Bosc, A. Labeyrie, L. Koechlin, S. Saha, Nature 342, 520 (1989)

[83] D. Mourard, J.D. Monnier, A. Meilland, D. Gies, F. Millour, M. Benisty, X. Che, E.D. Grundstrom, R. Ligi, G. Schaefer et al., A\&A 577, A51 (2015), 1503.03423

[84] R. Nan, Introduction to FAST: five hundred meter Aperture Spherical radio Telescope, in Ground-based and Airborne Telescopes II (2008), Vol. 7012, p. 70121E

[85] D. Mourard, A. Labeyrie, T. Lepine, P.D. Nunez, B. Tregon, Z. Xie, Experimental Astronomy (2018) 


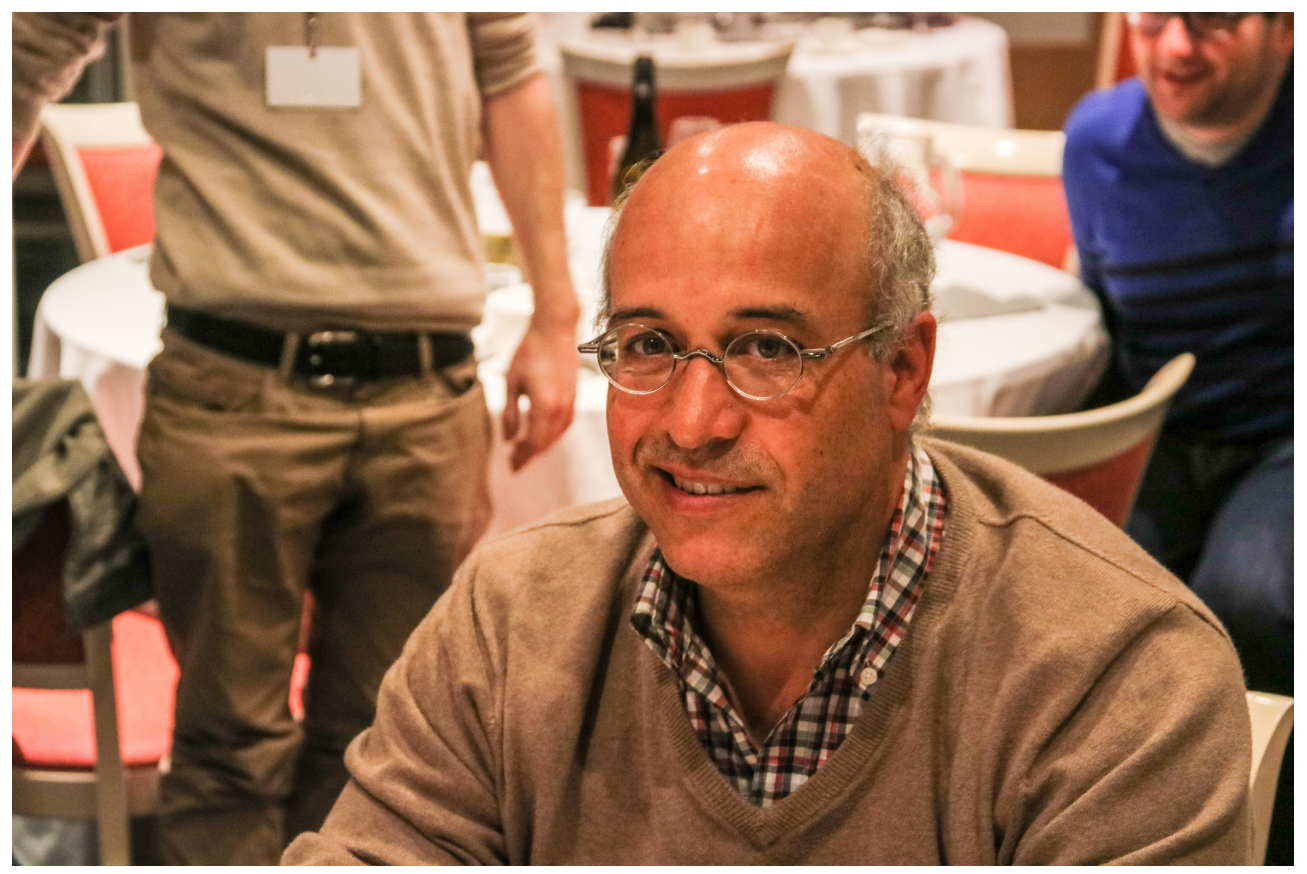

Figure 121. Denis Mourard, after his enlightning lecture, relaxing during the school dinner. 


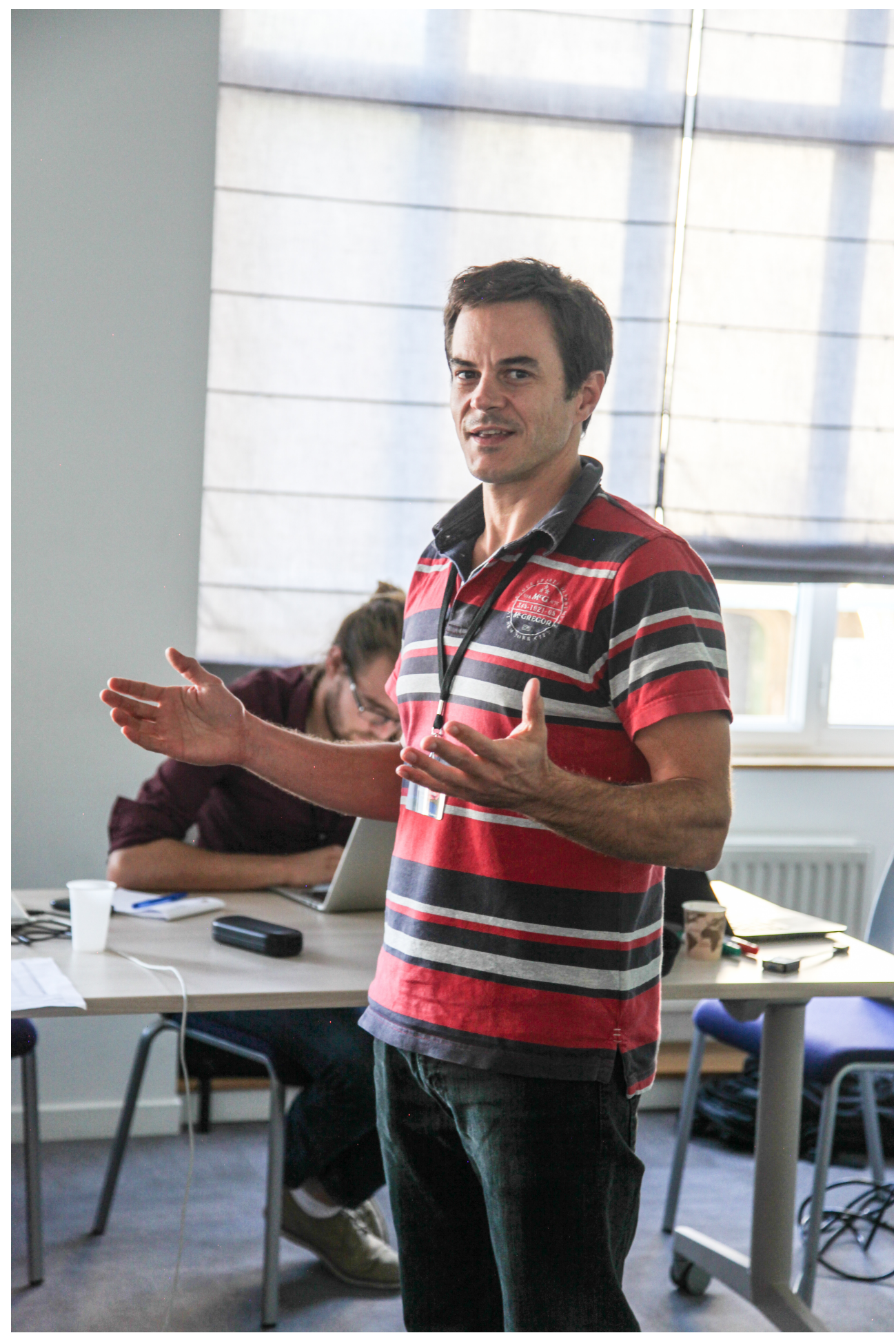

Figure 122. Nicolas Nardetto, guiding the students across the week of lectures. 


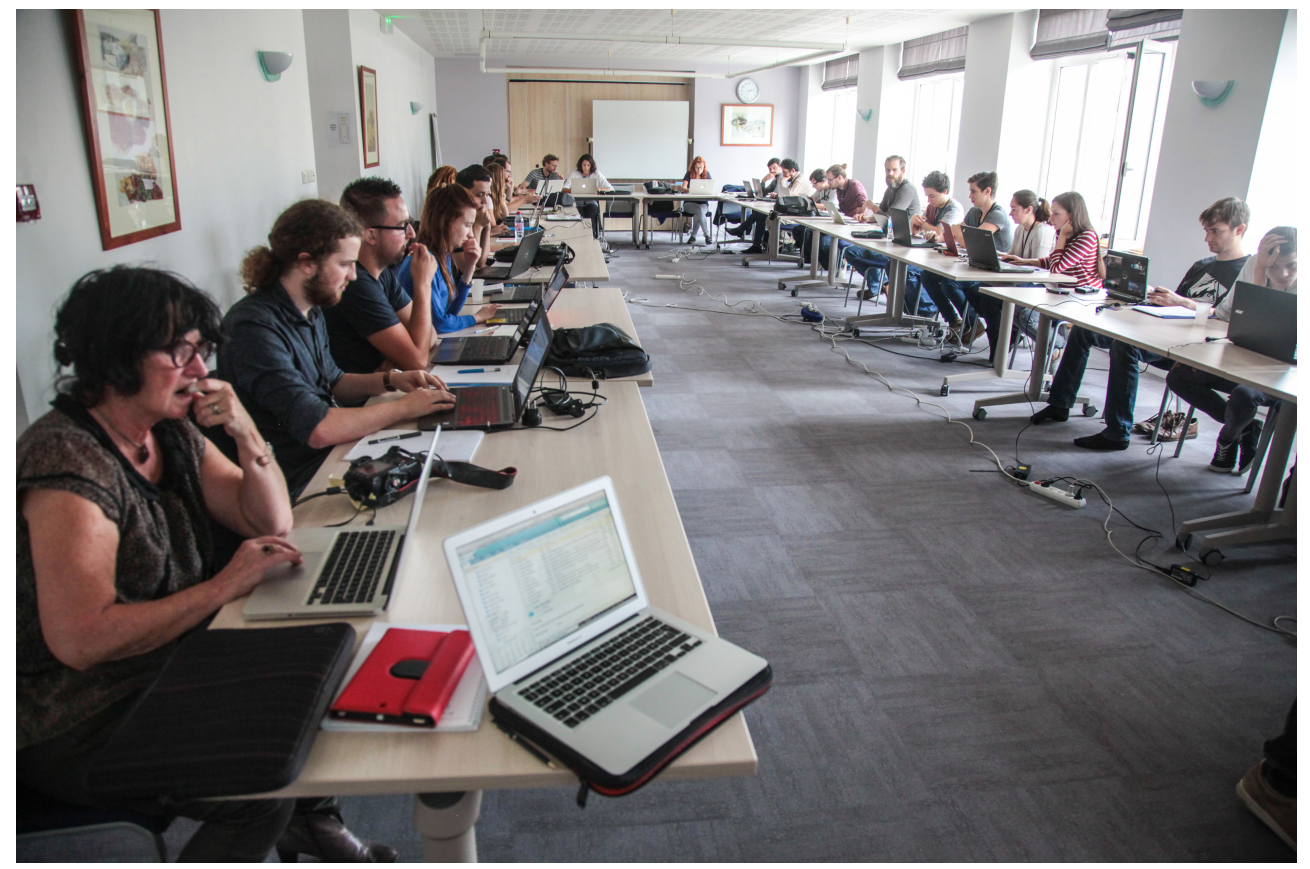

Figure 123. Students working during a practical session.

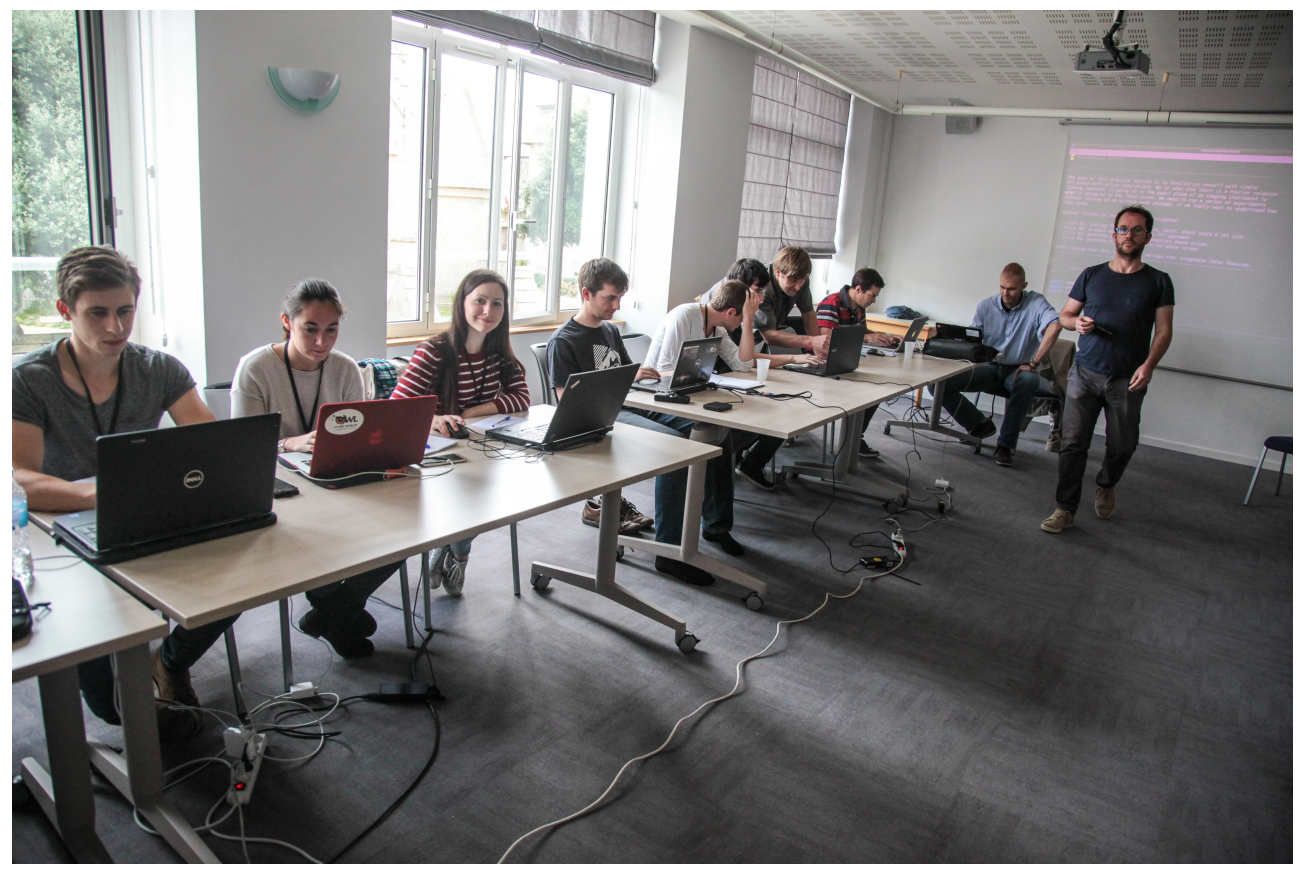

Figure 124. Hands-on session organized by Eric Lagadec to reduce SPHERE/VLT data 


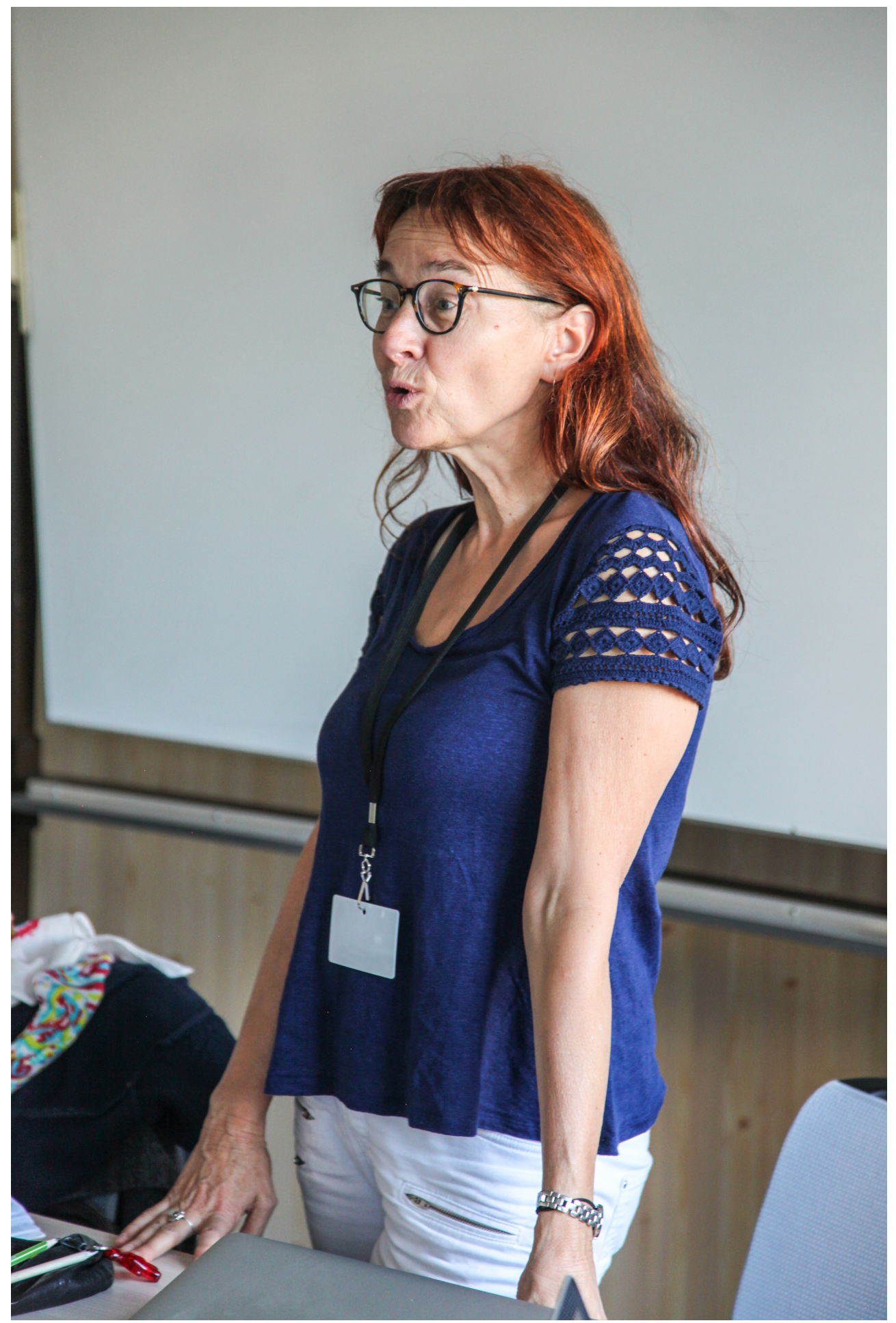

Figure 125. Yveline Lebreton, enjoying a hands-on session. 


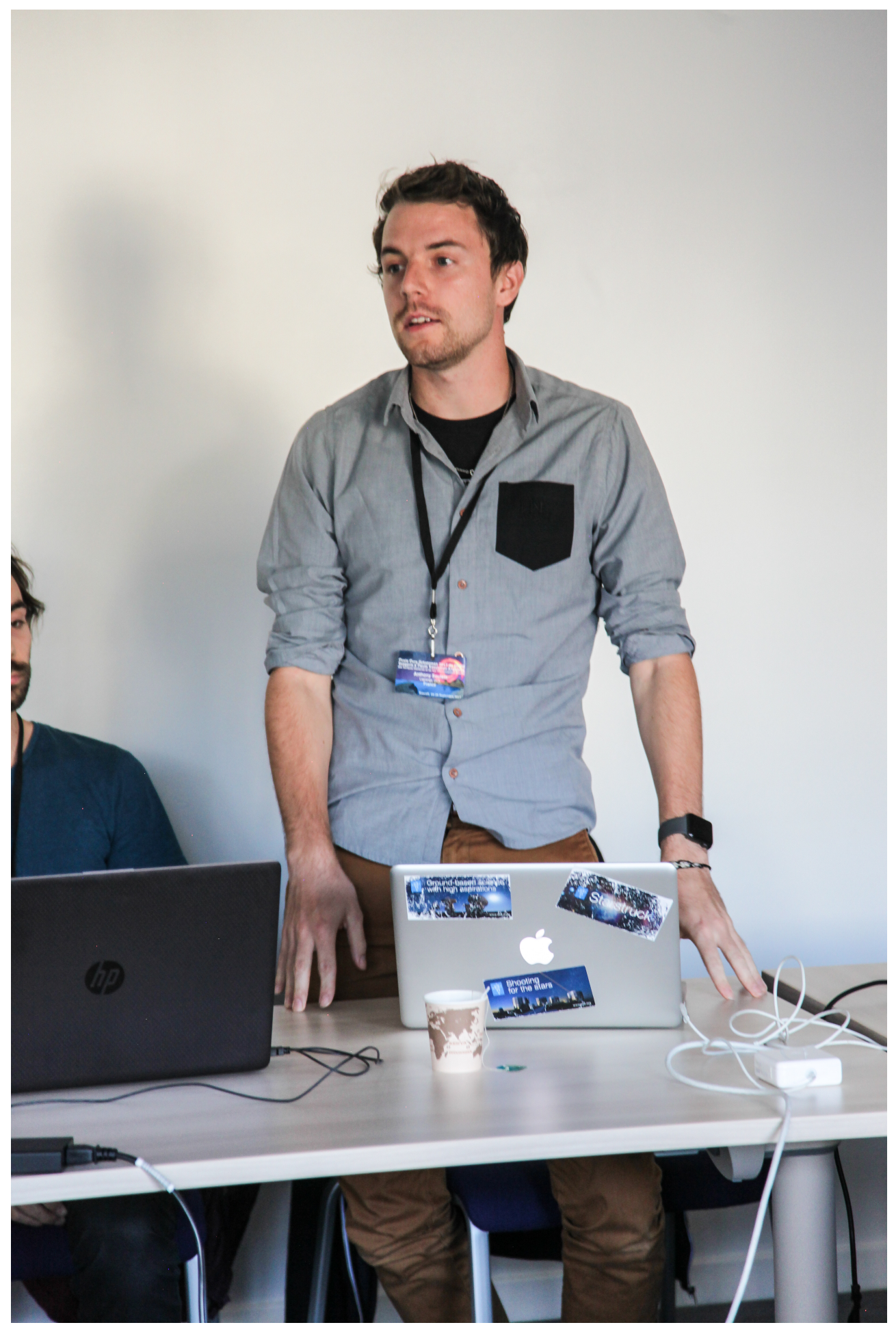

Figure 126. Anthony Soulain, helping Eric Lagadec during the SPHERE data reduction practical session. 


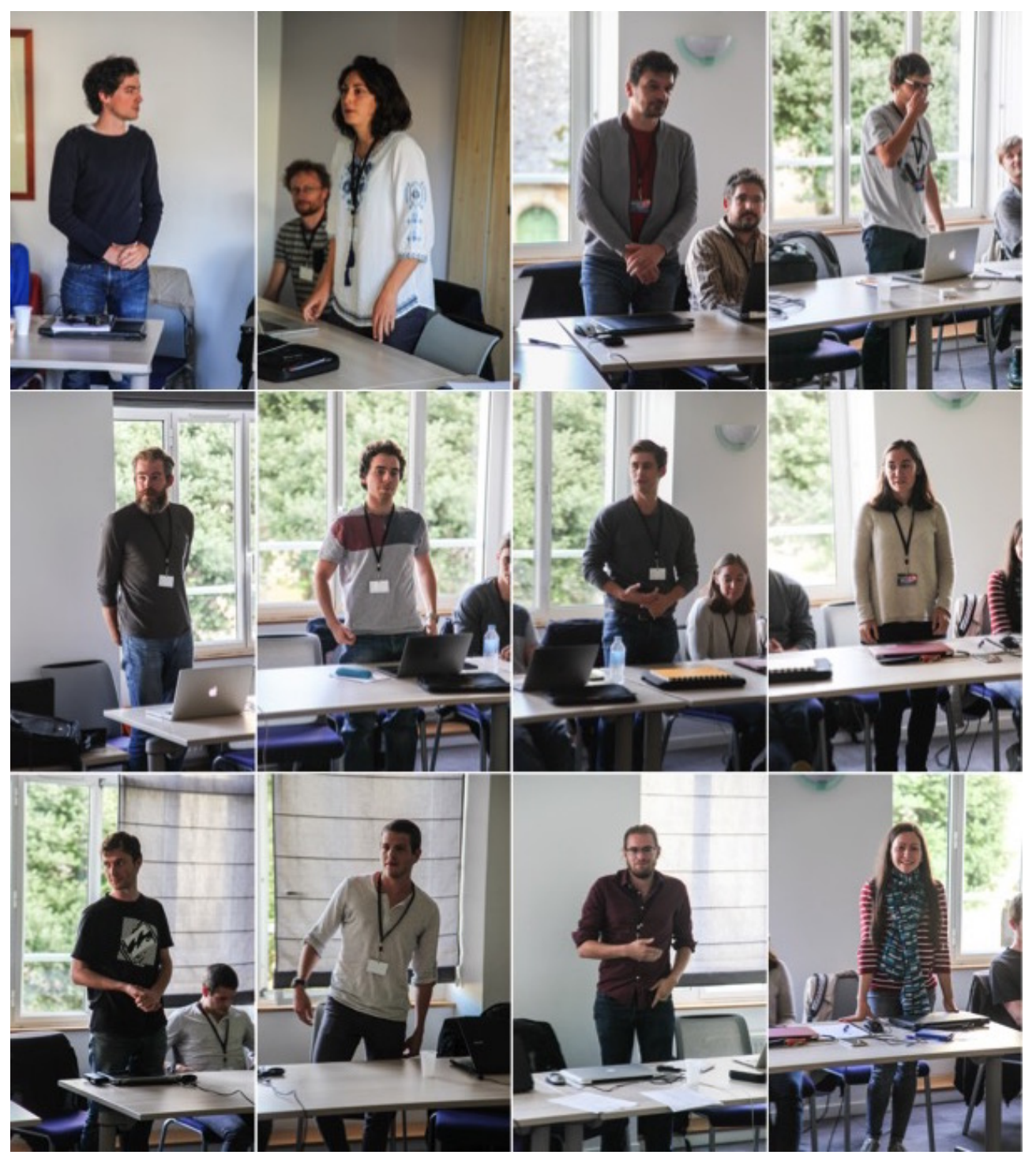

Figure 127. Gallery of the school's attendees 


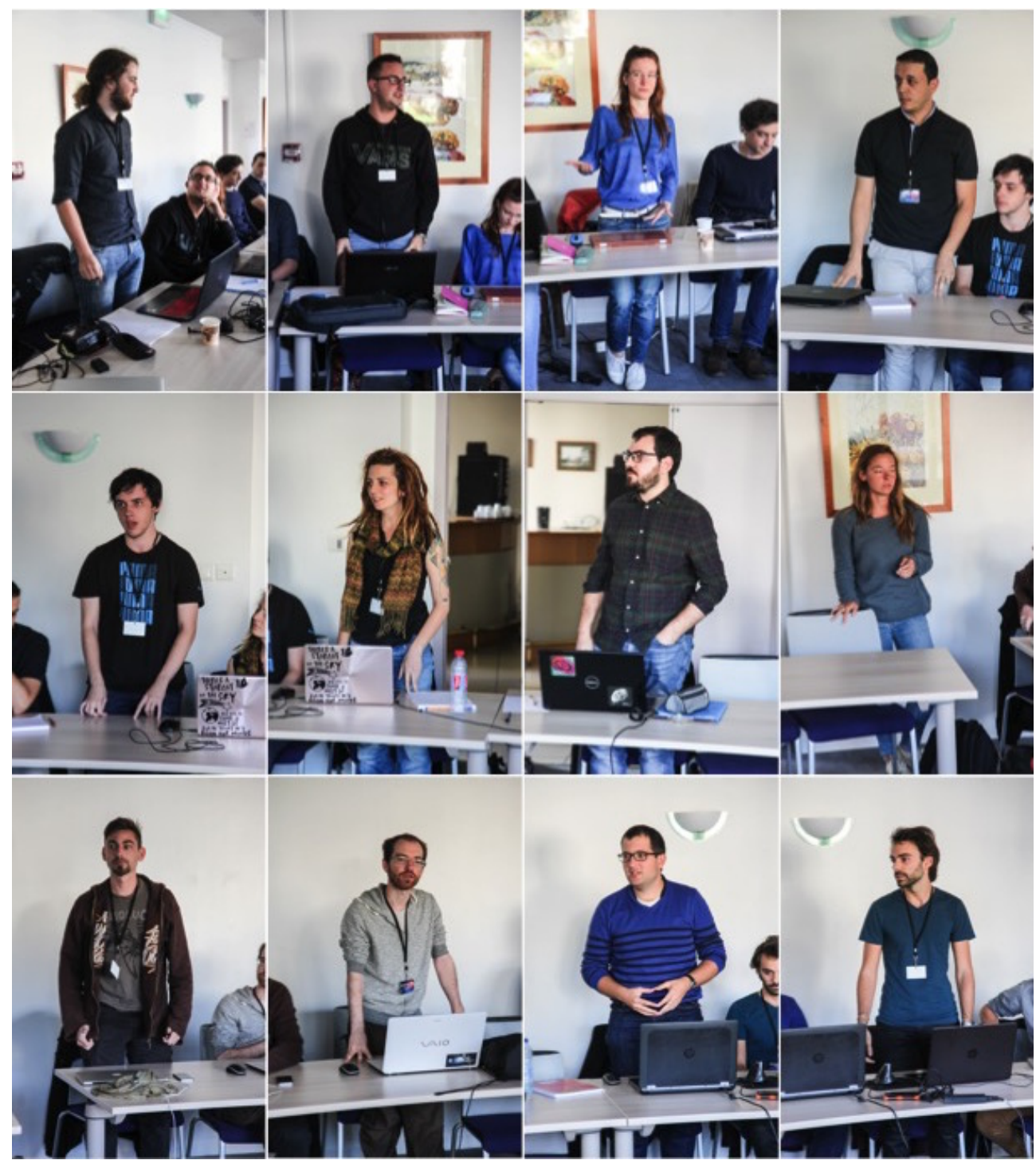

Figure 128. Gallery of the school's attendees. 


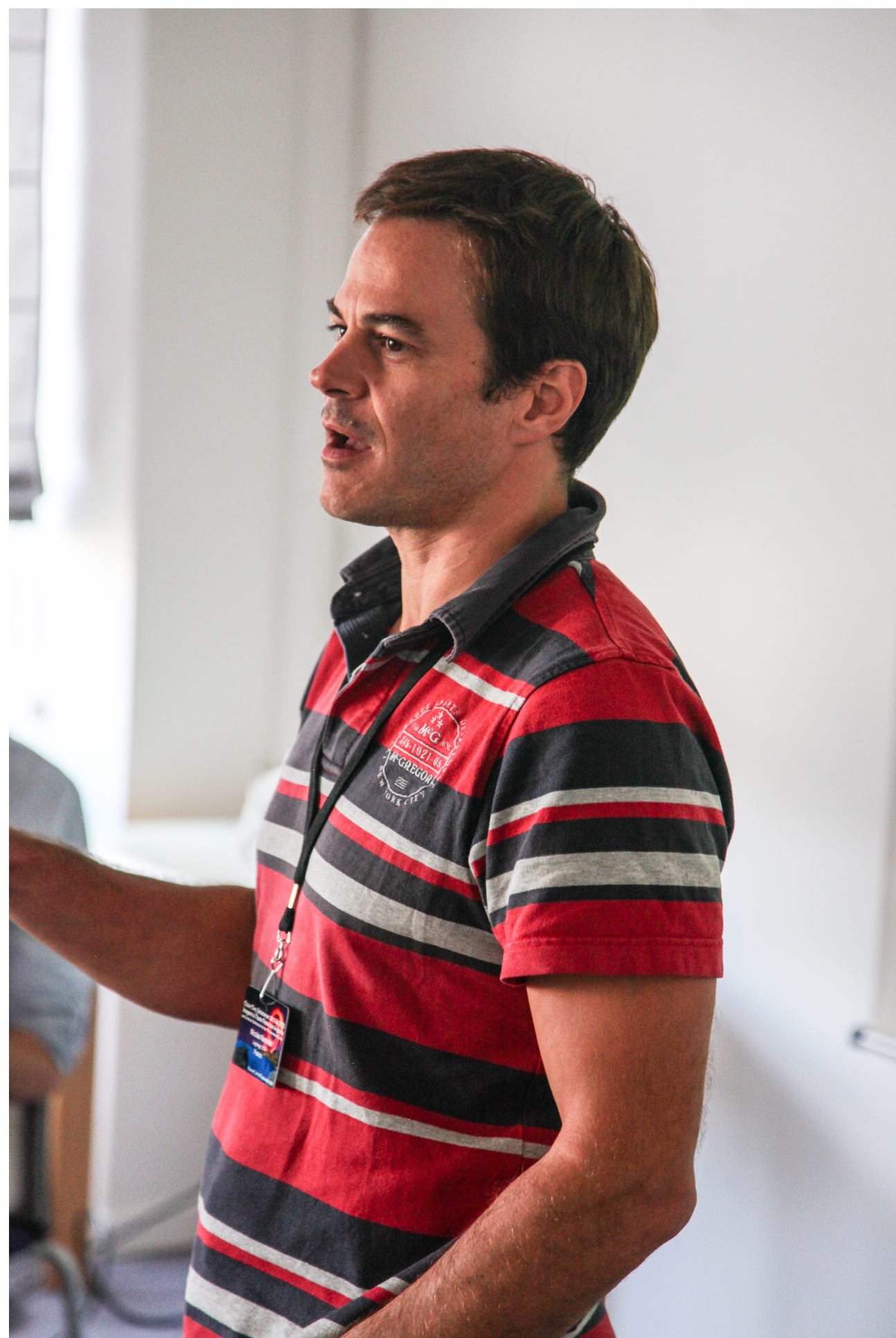

Figure 129. Nicolas Nardetto, working on the organisation of the sessions. 


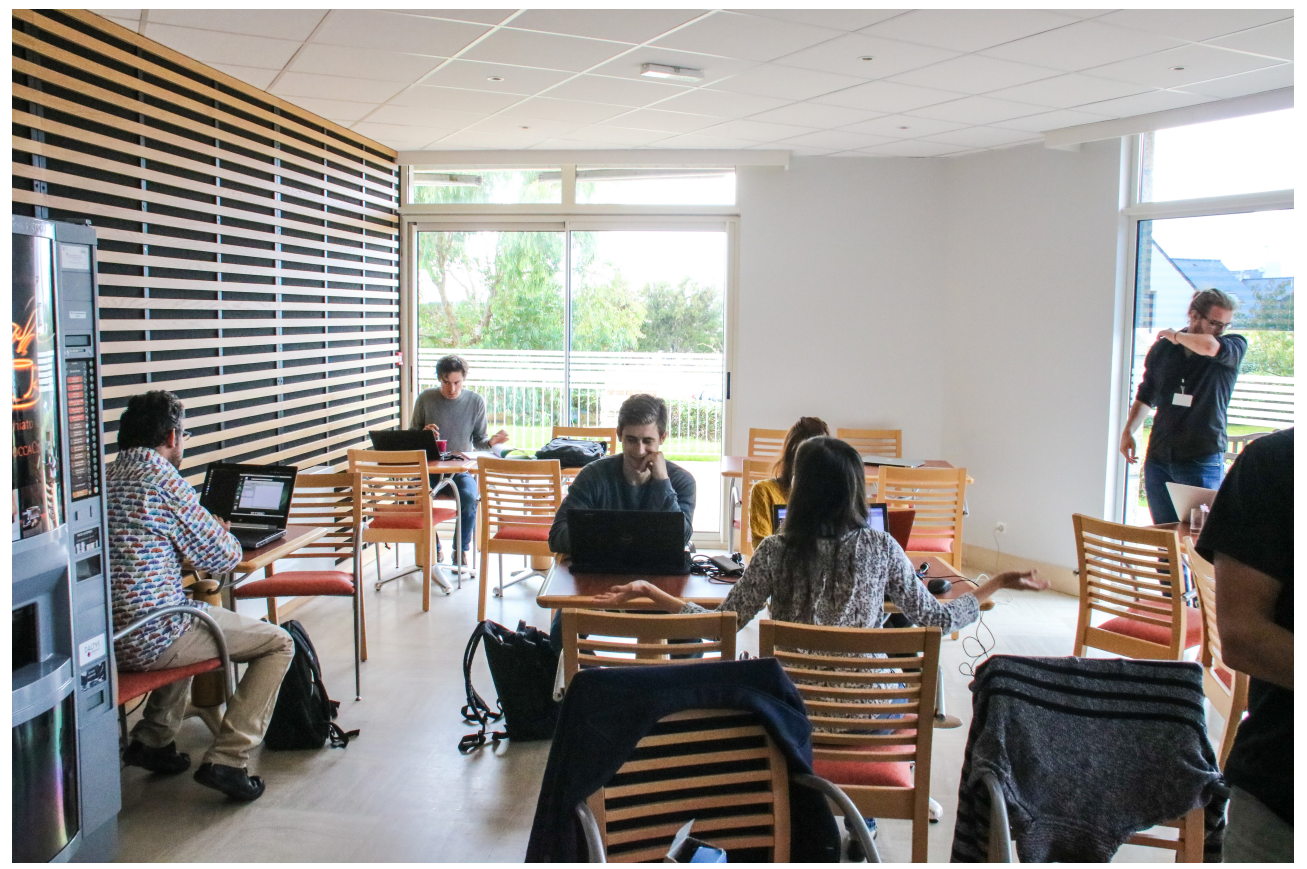

Figure 130. Participants working in the lobby of the hotel

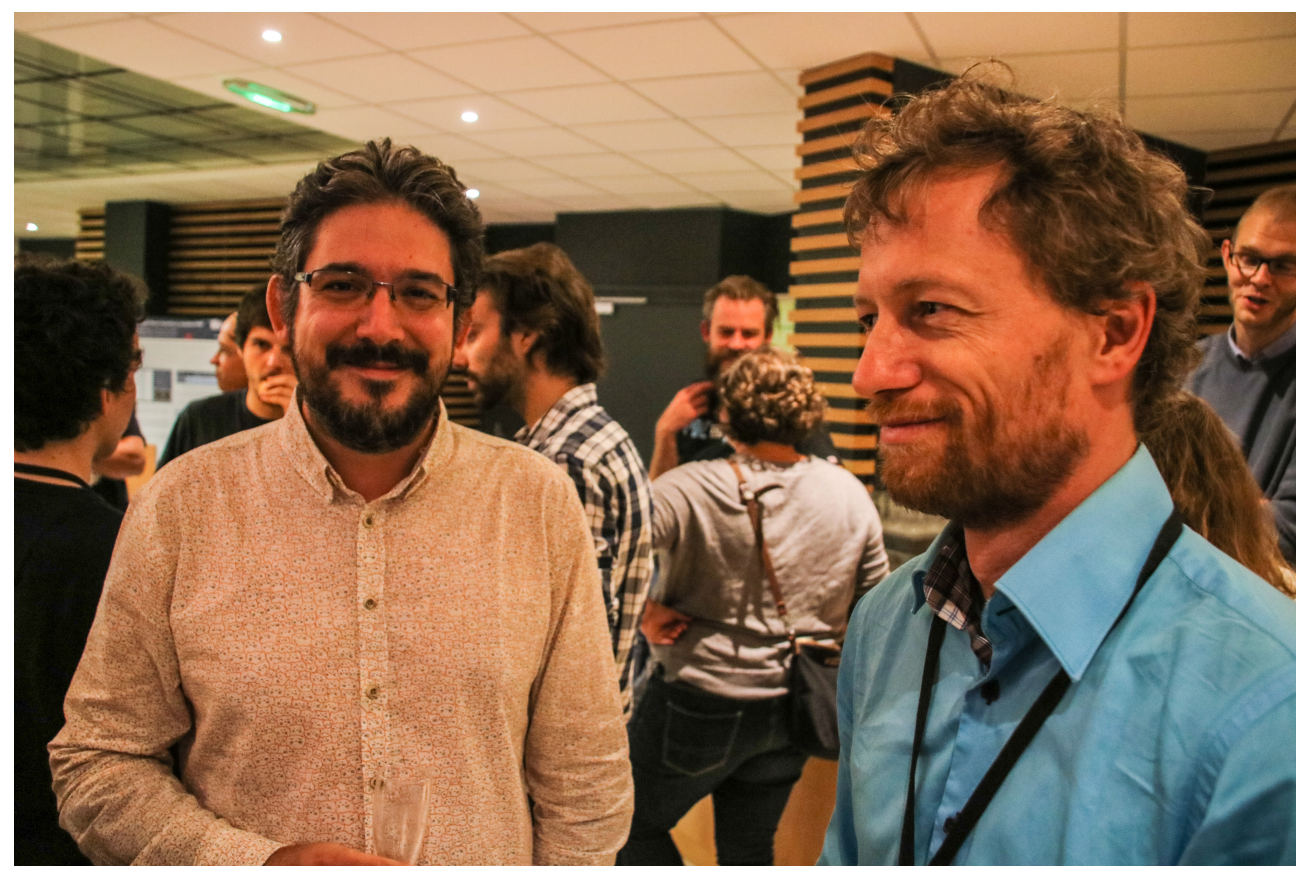

Figure 131. Florentin Millour and Aurélien Crida, enjoying the poster session. 


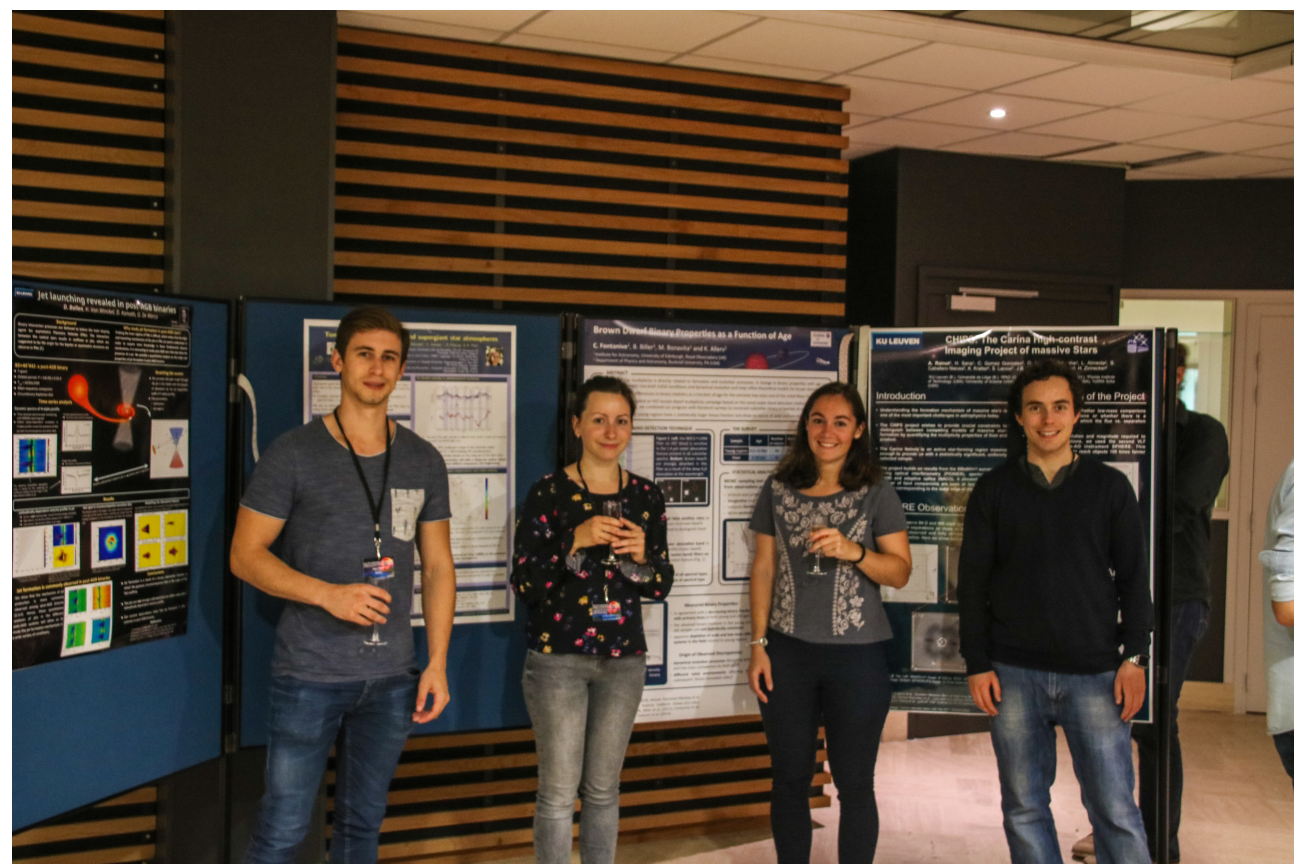

Figure 132.

Dylan Bollen, Katerina Kravchenko, Clémence Fontanive and Alan Rainot, presenting their posters.

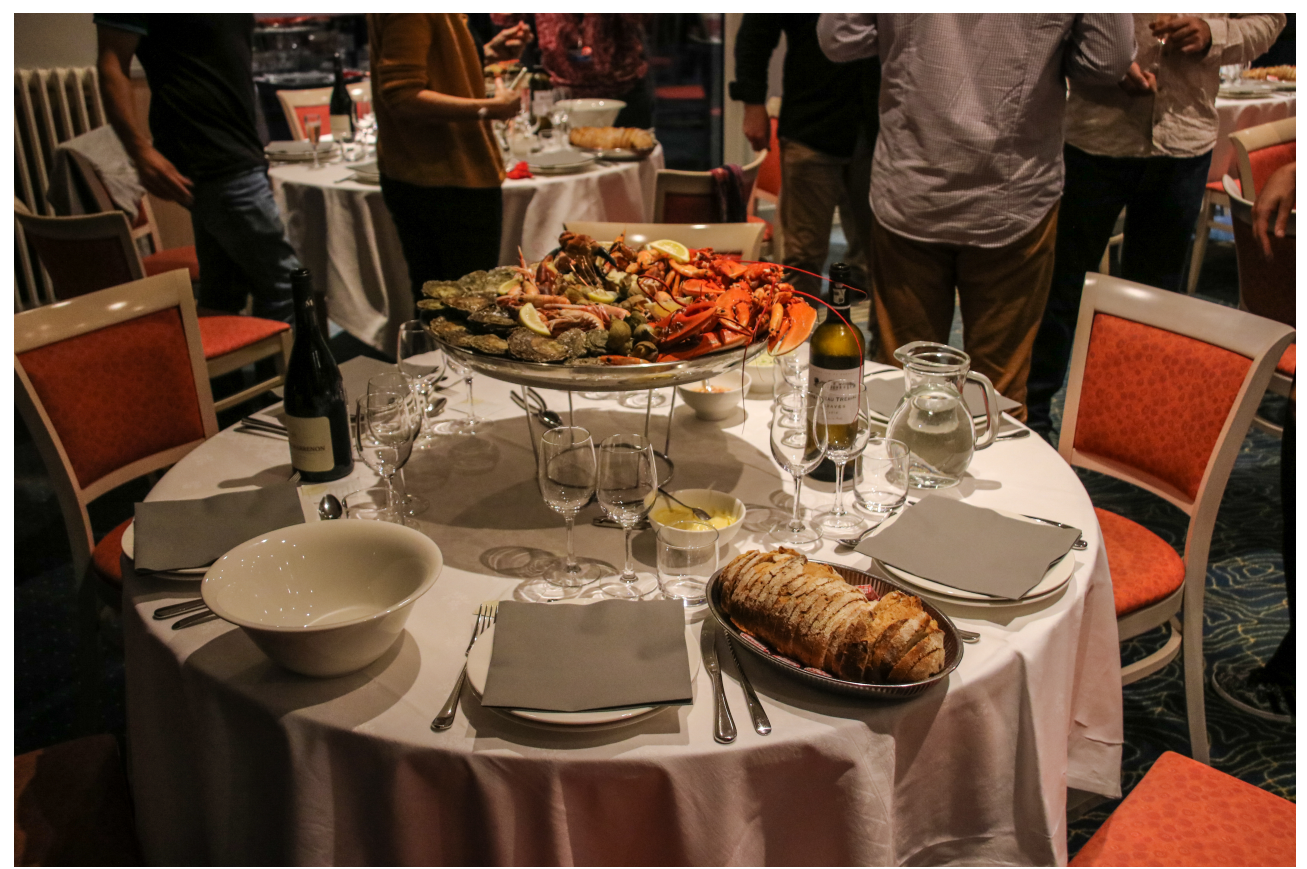

Figure 133. The school's dinner is ready, with great seafood from Brittany! 


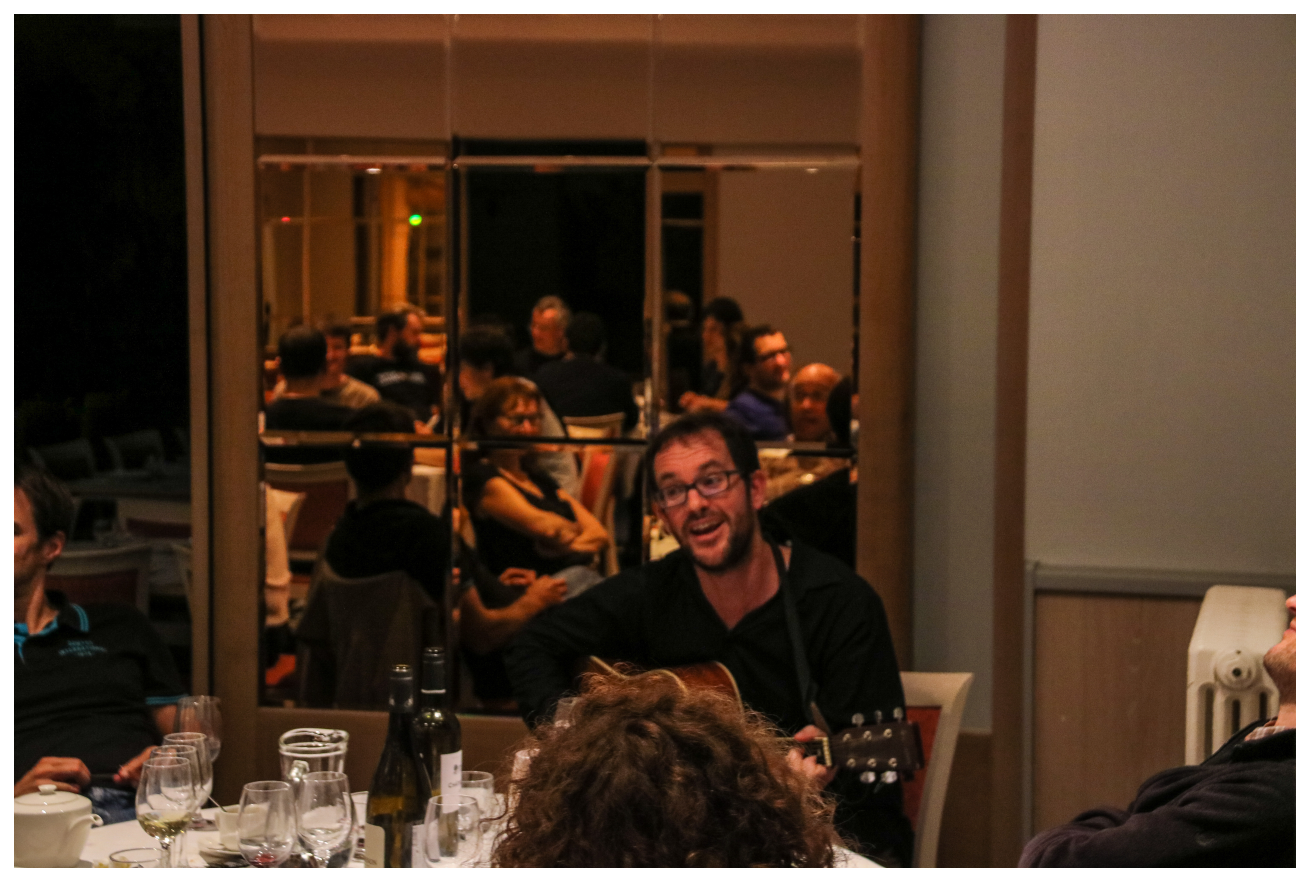

Figure 134. Improvised concert during the dinner.

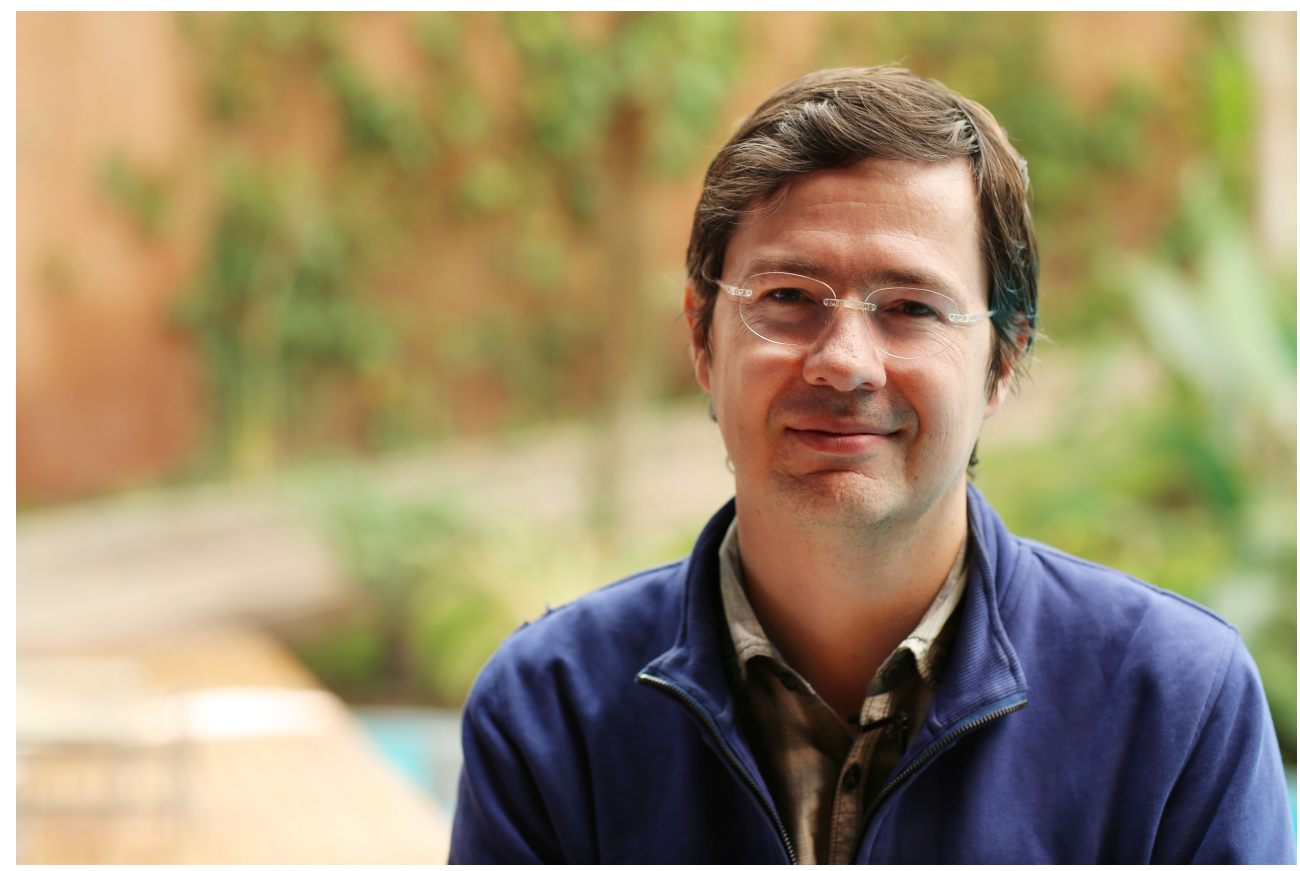

Figure 135. Pierre Kervella gave us an excellent seminar on the first GRAVITY/VLTI results. 


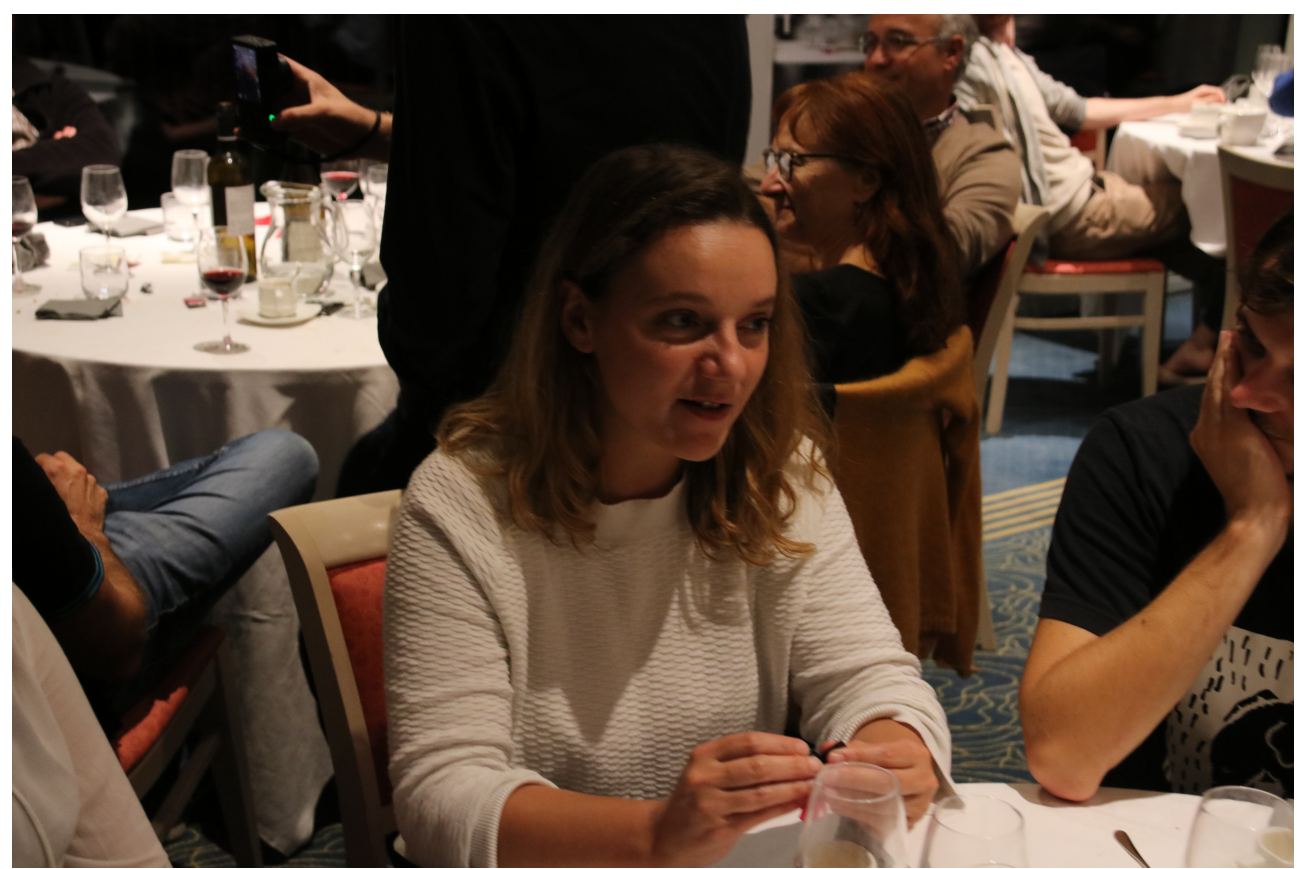

Figure 136. Annaëlle Maury relaxing after her excellent lecture on radio interferometry.

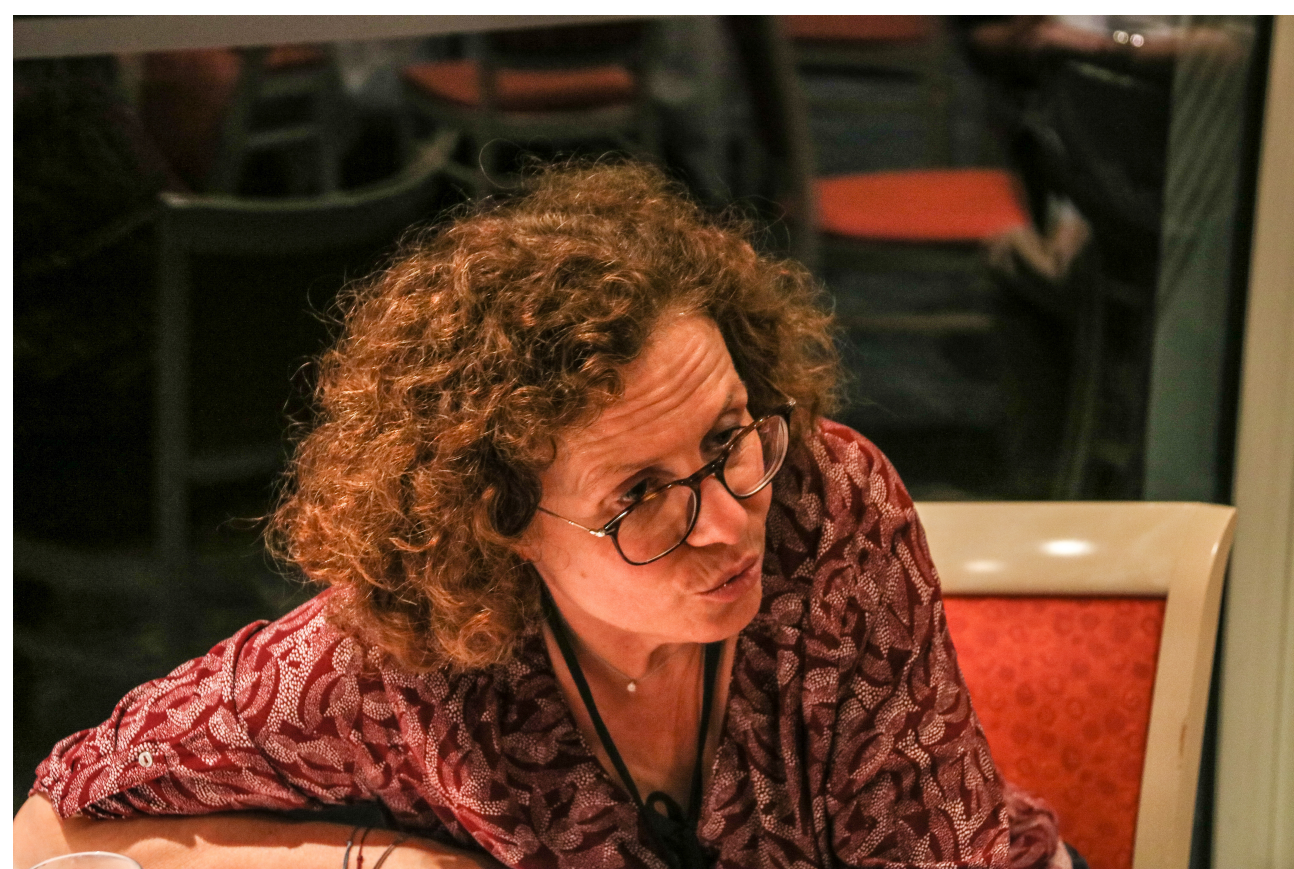

Figure 137. Anne-Marie Lagrange relaxing after her excellent lecture and enjoying being in Brittany. 